\title{
Frequency Domain Channel Estimation and Equalization for Broadband Wireless Generalized Multicarrier Systems
}

by

Chan-Tong Lam

A thesis submitted to the Faculty of Graduate Studies and Research in partial fulfillment of the requirements for the degree of Doctor of Philosophy.

Ottawa-Carleton Institute for Electrical and Computer Engineering

Faculty of Engineering

Department of Systems and Computer Engineering

Carleton University

Ottawa, Ontario, Canada

August, 2007

Copyright (C) Chan-Tong Lam, August, 2007 


$\begin{array}{ll}\begin{array}{l}\text { Library and } \\ \text { Archives Canada }\end{array} & \begin{array}{l}\text { Bibliothèque et } \\ \text { Archives Canada }\end{array} \\ \begin{array}{l}\text { Published Heritage } \\ \text { Branch }\end{array} & \begin{array}{l}\text { Direction du } \\ \text { Patrimoine de l'édition }\end{array} \\ \begin{array}{l}\text { 395 Wellington Street } \\ \text { Ottawa ON K1A ON4 }\end{array} & \begin{array}{l}\text { 395, rue Wellington } \\ \text { Ottawa ON K1A ON4 } \\ \text { Canada }\end{array}\end{array}$

Your file Votre référence ISBN: 978-0-494-33497-3 Our file Notre référence ISBN: $978-0-494-33497-3$

NOTICE:

The author has granted a nonexclusive license allowing Library and Archives Canada to reproduce, publish, archive, preserve, conserve, communicate to the public by telecommunication or on the Internet, loan, distribute and sell theses worldwide, for commercial or noncommercial purposes, in microform, paper, electronic and/or any other formats.

The author retains copyright ownership and moral rights in this thesis. Neither the thesis nor substantial extracts from it may be printed or otherwise reproduced without the author's permission.
AVIS:

L'auteur a accordé une licence non exclusive permettant à la Bibliothèque et Archives Canada de reproduire, publier, archiver, sauvegarder, conserver, transmettre au public par télécommunication ou par l'Internet, prêter, distribuer et vendre des thèses partout dans le monde, à des fins commerciales ou autres, sur support microforme, papier, électronique et/ou autres formats.

L'auteur conserve la propriété du droit d'auteur et des droits moraux qui protège cette thèse. $\mathrm{Ni}$ la thèse ni des extraits substantiels de celle-ci ne doivent être imprimés ou autrement reproduits sans son autorisation.
In compliance with the Canadian

Privacy Act some supporting forms may have been removed from this thesis.

While these forms may be included in the document page count, their removal does not represent any loss of content from the thesis.
Conformément à la loi canadienne sur la protection de la vie privée, quelques formulaires secondaires ont été enlevés de cette thèse.

Bien que ces formulaires aient inclus dans la pagination, il n'y aura aucun contenu manquant.

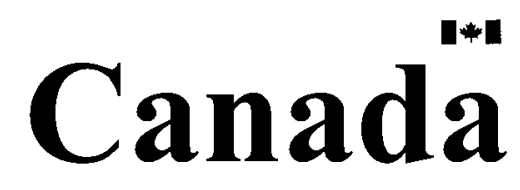


To my parents

Kin-Meng Lam and Lai-Chu Sun

and to my family

Zoe S.-M. Chan and Chloe H.-C. Lam

Reproduced with permission of the copyright owner. Further reproduction prohibited without permission. 


\section{Abstract}

Using the generalized multicarrier approach, we propose two in-band frequency domain multiplexed (FDM) pilot schemes, namely frequency expanding technique (FET) and frequency domain superimposed pilot technique (FDSPT), for single-carrier (SC) systems in broadband wireless channels. The frequency domain equalizers for SC signals with FDM pilots are also developed. FDM pilots result in higher peak to average power ratio (PAPR) and required power backoff of an SC signal, comparing with that of an SC signal without FDM pilots, although the PAPR and required power backoff are still lower than that of an orthogonal frequency division multiplexing (OFDM) signal. We then propose a PAPR reduction technique for SC signals with FDM pilots, using a set of cyclic-shifted Chu sequences. The frame error rate (FER) performance of a coded system for our proposed frequency domain channel estimators using time domain multiplexed (TDM) and FET pilots are similar, while using FDSPT pilots loses about $1 \mathrm{~dB}$ with respect to that of using FET or TDM pilots. To further improve the performance of SC systems using FDSPT pilots, we propose a frequency domain decision-directed interference cancellation technique, with which the loss of using FDSPT pilots reduces by $0.5 \mathrm{~dB}$.

Multiple frames can be used to improve channel estimation for chunk size and full bandwidth systems at the expense of slightly increasing the complexity. FDSPT pilots can also be used for OFDM systems with channel coding. We compare the performance of the proposed channel estimators for SC and OFDM systems using TDM and FDM pilot schemes in terms of pilot overhead, cyclic prefix overhead, the required power backoff for the high power amplifier (HPA) and the FER. Taking into account the required power backoff of a HPA, a serial modem with FDM pilots could be designed with a power amplifier with about $2 \mathrm{~dB}$ lower maximum power rating than that of a corresponding OFDM modem. We also study the impact of channel estimation errors on the MSE of the equalizer output. Finally, we compare the performance between interleaved frequency division multiple access and direct sequence code division multiple access systems with frequency impairments. 


\section{Acknowledgements}

First of all, I would like to give my sincere thanks to my thesis supervisors, Professor David D. Falconer and Professor Florence Danilo-Lemoine, for their inspiration, encouragement and patience throughout the study of my thesis. I am deeply influenced by their endless passion for wireless communication research and their mentorship for academic and industrial research, which are for sure to be beneficial for my future research career. I am indebted to David's flexible arrangement for my research environment so that I can spend more time with my family in Macau.

I am grateful to Dr. Benjamin Ng at Radiospire Networks, Boston, U.S.A. and Professor Rui Dinis at CAPS-IST, Technical University of Lisbon, Portugal for their guidance and insightful discussions. I am also grateful to Dr. Gunther Auer at DoCoMo Euro-Labs, Munich, Germany for his helpful discussions.

I would like to think my friends Benjamin Ng, Hua Xiao, Xiao-Ning Li, Jin-Shi Qiu, Thomas Cheng, Cindy Yu and Paul Ng for making my stay at Carleton enjoyable. Dinners together, soccer games, skating on Rideau Canal, tennis games and chatting are all memorable. I would also like to thank my colleagues, Maryam Sabbaghian and Fayyaz A. Siddiqui for letting me learn from them and be close to them during my graduate years at Carleton.

I would like to thank the departmental office staffs for helping me at various points throughout my degree program. In particular, I would like to thank Blazanka Power, Darlene Hebert, Anna Lee and Jennifer Poll for their administrative support.

Finally, I would like to acknowledge my beloved wife, grandparents, parents, my sister and my uncles for their continuous love and support. They have helped me in every possible way to pursue and achieve my goals. I am forever indebted to them.

Chan-Tong Lam, August 17, 2007 Ottawa, Canada 


\section{Table of Contents}

Abstract iv

Acknowledgements $\quad$ v

Table of Contents vi

List of Figures $\quad$ xi

List of Tables $\quad$ xix

1 Introduction 1

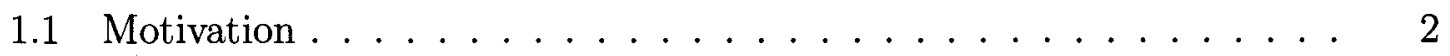

1.2 Objectives and Scope . . . . . . . . . . . . . 3

1.3 Thesis Contributions $\ldots \ldots \ldots \ldots \ldots \ldots \ldots$

1.4 Thesis Overview . . . . . . . . . . . . . . . . 9

2 Background and Literature Review 13

2.1 Generalized Multicarrier (GMC) Systems . . . . . . . . . . . . 14

2.2 Single-Carrier versus OFDM Systems . . . . . . . . . . . . . 14

$2.2 .1 \quad$ Power Amplifier Efficiency . . . . . . . . . . . . . . 15

2.2.2 Flexibility of Spectrum Assignment _ . . . . . . . . 16

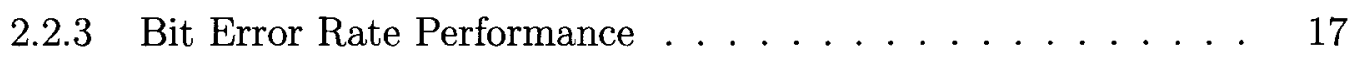

2.2.4 Quantization Effects and Phase Noise Sensitivity . . . . . 18

2.2.5 Advantages of SC Modulation in the Uplink . . . . . . . . 19 
2.3 System Descriptions _ . . . . . . . . . . . . . . . . . . . . . 19

2.3.1 GMC Transmitter and Receiver with FDM Pilots . . . . . . 21

2.3 .2 IFDMA Training Signal $\ldots \ldots \ldots \ldots 22$

2.3.3 Time-varying Multipath Channel Model . . . . . . . . . . . 24

2.3.4 Simulation System Parameters . . . . . . . . . . . . . 27

2.4 Frequency Domain Channel Equalization for SC Systems . . . . . . 30

2.4.1 Linear MMSE Channel Equalization . . . . . . . . . 30

2.4.2 Iterative Block Soft Decision Feedback Equalization . . . . . . 31

2.5 Overview of Channel Estimation for SC Systems . . . . . . . . . . 34

2.5.1 TDM Pilot Arrangements . . . . . . . . . . . . 35

2.5.2 Time Domain Least Square (LS) and LMMSE Channel Estimator 36

2.6 Overview of Channel Estimation for OFDM Systems _ . . . . . . 37

2.6.1 FDM Pilot Arrangements . . . . . . . . . . . . . . 39

2.6.2 DFT-Based Channel Estimator . . . . . . . . . . . . . 40

2.6.3 Cascaded 1D-FIR Wiener Channel Estimator . . . . . . . 41

2.6.4 Iterative Decision-Directed Channel Estimation . . . . . . 44

2.7 Challenges of Designing Channel Estimation Techniques for Uplink SC

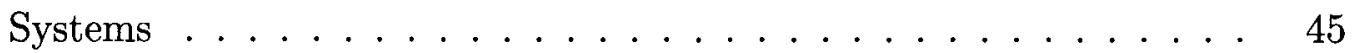

3 Effects of Channel Estimation Errors on BER Performance of SC $\begin{array}{ll}\text { Systems } & \mathbf{4 7}\end{array}$

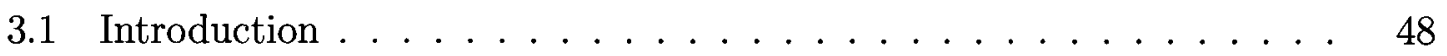

3.2 Analytical Approach . . . . . . . . . . . . . . . . . . 49

$3.2 .1 \quad$ Calculation of $S N R_{e q z} \ldots \ldots \ldots \ldots \ldots$

3.2.2 Determination of $J_{A} \triangleq E\left\{\left|\epsilon_{m}\right|^{2}\right\} \quad \ldots \ldots \ldots \ldots \ldots$

3.2.3 Determination of $E\{\kappa\}$ and $E\left\{|\kappa|^{2}\right\} \ldots \ldots \ldots \ldots$

3.3 Semi-Analytical Approach . . . . . . . . . . . . . . . . 54

3.4 Simulation Results . . . . . . . . . . . . . . . . . . . 58 
3.4.1 MSE of Equalizer Output . . . . . . . . . . 58

3.4 .2 BER Estimation $\ldots \ldots \ldots$. . . . . . . . . . . 58

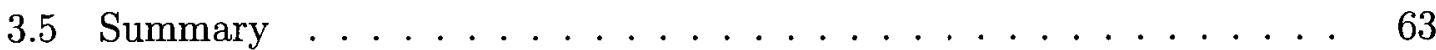

4 Design and Equalization of SC Signals with FDM Pilots 64

4.1 Introduction . . . . . . . . . . . . . . . 64

4.2 Analysis of SC Signals with FDM Pilots . . . . . . . . . . . 66

4.2.1 Frequency Domain Superimposed Pilot Technique . . . . . . 66

4.2.2 Frequency Expanding Technique (FET) . . . . . . . . . . . 69

4.2.3 PAPR and Power Backoff Considerations . . . . . . . . . 71

4.3 Channel Equalization of SC Signals with FDM Pilots . . . . . . . . . 73

4.3.1 Frequency Domain LMMSE Equalization . . . . . . . . . . 74

4.3.2 Frequency Domain IBSDFE Equalization . . . . . . . . . . 75

4.4 Interference Cancellation of SC Signals with FDSPT pilots . . . . . 78

4.5 Simulation Results . . . . . . . . . . . . . . . 81

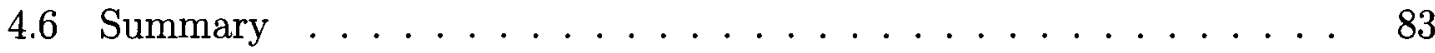

5 Channel Estimation using FDM Pilots for Full Bandwidth Systems 85

5.1 Introduction . . . . . . . . . . . . . . . 86

5.2 Non-Iterative Channel Estimation for SC Signals with FDM Pilots . . 87

5.2.1 Hybrid Channel Estimator: FFT-LLSLF . . . . . . . . . . 87

5.2.2 Hybrid Channel Estimator: FFT-Wiener . . . . . . . . . . 88

5.3 Iterative Channel Estimation for SC Signals with FDM Pilots . . . . 88

5.3.1 Proposed Low Complexity Frequency Domain IDDCE . . . . . 89

5.3.2 Issue of Noise Enhancement . . . . . . . . . . . . 90

5.3.3 Optimum Technique: MMSE Estimator . . . . . . . . . . 90

5.3.4 Sub-optimum Low Complexity Technique: The Frequency Replacement Algorithm . . . . . . . . . . . . . . 91

5.3.5 Wiener $2 \times 1 \mathrm{D}$ Filtering of Raw Estimates . . . . . . . . . 92

viii 
5.3.6 Determination of the Best Value of $\lambda \ldots \ldots 93$

5.4 Performance Evaluation . . . . . . . . . . . . . 93

5.4 .1 Averaged MSE ..................... 94

5.4 .2 Frame Error Rate (FER) . . . . . . . . . . . . 96

5.4.3 Effects of Mis-Matched Delay Spread and Vehicle Speed . . . 99

5.5 Comparison of OFDM and SC Systems using TDM and FDM Pilots . 103

5.6 Summary ............................. 107

6 Channel Estimation using FDM Pilots for Chunk and Sub-chunk $\begin{array}{ll}\text { Size Systems } & 109\end{array}$

6.1 Introduction . . . . . . . . . . . . . . . . 109

6.2 Description of Chunk and Sub-chunk Size Systems . . . . . . . . . . 111

6.2.1 Chunk Size Systems _. . . . . . . . . . . . . . 111

6.2 .2 Sub-chunk Systems . . . . . . . . . . . . . . 113

6.3 Chunk and Sub-chunk Size Channel Estimation over One Frame . . . 115

6.3.1 Pilot Arrangements for Sub-chunk Size Systems . . . . . . . . 115

6.3.2 Non-iterative Channel Estimation . . . . . . . . . . . . 116

6.3.3 Iterative Decision-Directed Channel Estimation . . . . . . . 118

6.4 Channel Estimation for Chunk and Sub-chunk Size Systems over Multiple Frames . . . . . . . . . . . . . . . . . . . . . 118

6.5 Performance Evaluation . . . . . . . . . . . . . . 120

6.5.1 Averaged MSE .................. 120

6.5.2 Effects of Vehicle Speed on MSE Performance of B-IFDMA Systems over Multiple Frames . . . . . . . . . . . . 123

6.5.3 Frame Error Rate (FER) . . . . . . . . . . . 125

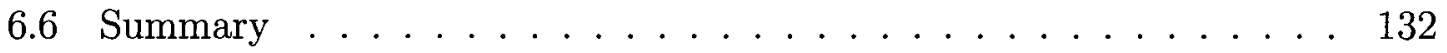

7 PAPR Reduction using FDM Pilot Sequences 133

7.1 Introduction . . . . . . . . . . . . . . . 133

ix 
7.2 Pilot-Aided PAPR Reduction for SC Signals . . . . . . . . . . . . 134

7.3 PAPR Reduction Capability of Walsh-Hadamard and Cyclic-shifted Chu Sequences ........................ 137

7.4 Effects of Selection Size and Rules . . . . . . . . . . . . 138

7.5 Effects on Out-of-Band Distortion after HPA . . . . . . . . . . . 140

7.6 Summary and Discussions . . . . . . . . . . . . . 145

8 Conclusions, Design Recommendations and Future Work 146

8.1 Conclusions .......................... 146

8.2 Design Recommendations . . . . . . . . . . . . . . . 148

8.3 Future Work . . . . . . . . . . . . . . . . 149

A Details of Derivation $\quad 151$

A.1 Derivation of $(3.11) \ldots \ldots \ldots \ldots 15 \ldots \ldots$

A.2 Derivation of $(3.15) \ldots \ldots \ldots \ldots \ldots$

A.3 Derivation of $(4.4) \ldots \ldots \ldots \ldots$

B Performance Comparison of IFDMA and DS-CDMA Systems in the Presence of Frequency Offsets and Phase Noise $\quad 154$

B.1 Frequency Domain LMMSE Receiver for DS-CDMA and IFDMA Sys-

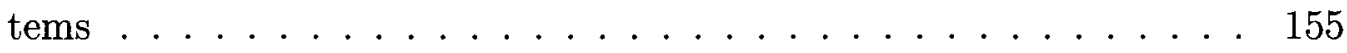

B.1.1 Frequency Domain LMMSE Receiver for DS-CDMA Systems . 155

B.1.2 Frequency Domain LMMSE Receiver for IFDMA Systems . . 156

B.2 Comparison of the DS-CDMA and IFDMA Systems without Frequency Impairments . . . . . . . . . . . . . . . . 157

B.2.1 Effect of Number of Users, $P_{u} \ldots \ldots 157$

B.2.2 Effect of Es/No . . . . . . . . . . . 160

B.2.3 Effect of Number of Antenna Elements, $N_{A} \ldots \ldots 160$ 
B.3 Analytical Results for Single-user IFDMA Systems with Constant Frequency Offsets and Wiener Phase Noise . . . . . . . . . . . 161

B.3.1 Analytical Results for Constant Frequency Offset . . . . . . . 164

B.3.2 Analytical Results for Wiener Phase Noise . . . . . . . . . 165

B.4 Comparison of IFDMA and DS-CDMA Multi-user Systems with Frequency Offset and Phase Noise . . . . . . . . . . . . . 168

B.4.1 Comparison of Systems with Constant Frequency Offset . . 169

B.4.2 Comparison of Systems with Phase Noise . . . . . . . . . . 171

B.5 Summary ............................ 175

$\begin{array}{lr}\text { Bibliography } & 176\end{array}$ 


\section{List of Figures}

1.1 WINNER's View of A Ubiquitous 4G Radio System Concept . . . . . 2

1.2 Relation of various Topics Considered in This Thesis . . . . . . . . . 10

2.1 Generalized Multicarrier Transmitter and Receiver Structure [21, Ch. 6] 15

2.2 Overall System Block Diagram . . . . . . . . . . . . . . . . 20

2.3 Definition of a Frame with Multiple Blocks . . . . . . . . . . . 20

2.4 GMC Transmitter and Receiver Structure with FDM Pilots . . . . . . 22

2.5 Magnitude Response of IFDMA Signals with $M_{c}=64, N_{c}=8$ and

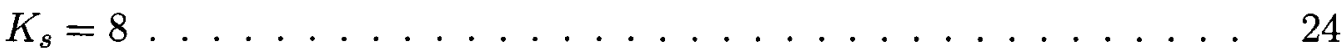

2.6 Structure of a Frequency Domain LMMSE Equalizer . . . . . . . . . 30

2.7 Structure of A Frequency Domain Iterative Block Soft Decision Feedback Equalizer . . . . . . . . . . . . . . . . . 31

2.8 TDM Pilot Arrangements with CP . . . . . . . . . . . 36

2.9 Typical Channel Estimation Techniques for OFDM Systems . . . . 37

2.10 Time-Frequency Rectangular Pilot Grid . . . . . . . . . . . . . 39

2.11 A General Structure of DFT-Based Channel Estimator . . . . . . . . 40

2.12 Iterative Decision-Directed Channel Estimator for OFDM Systems [56,

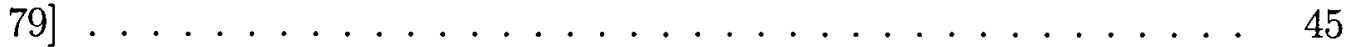

2.13 Illustration of Trade-offs among Different Requirements of the Challenges 46

3.1 Comparison of the CDF of the Real Part of $\Delta H_{\ell}$ from an FFT Estimator $(M=416$ and $N=52)$ and that of a Gaussian Random Variable with Zero Mean and a Variance of $\sigma_{\Delta H}^{2}=0.088$ at SNR $=10 \mathrm{~dB} \ldots 55$ 
3.2 Comparison of the CDF of the Real Part of $\Delta H_{\ell}$ from an FFT Estimator $(M=416$ and $N=52)$ and that of a Gaussian Random Variable with Zero Mean and a Variance of $\sigma_{\Delta H}^{2}=0.018$ at SNR $=20 \mathrm{~dB}$. .

3.3 Comparison of the CDF of the Real Part of $\Delta H_{\ell}$ from a Wiener Estimator $(M=416$ and $N=104)$ and that of a Gaussian Random Variable with Zero Mean and a Variance of $\sigma_{\Delta H}^{2}=0.045$ at $\mathrm{SNR}=10$

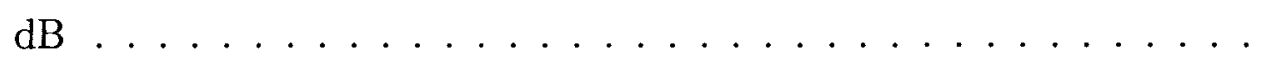

3.4 Comparison of the CDF of the Real Part of $\Delta H_{\ell}$ from a Wiener Estimator $(M=416$ and $N=104)$ and that of a Gaussian Random Variable with Zero Mean and a Variance of $\sigma_{\Delta H}^{2}=0.0051$ at SNR $=$

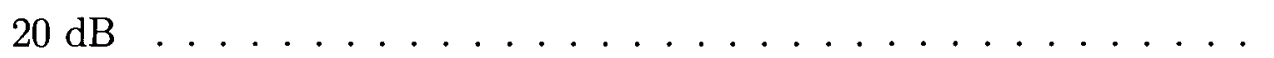

3.5 Comparison of Simulated and Analytical Results for MSE of Equalizer Output with Channel Estimation Errors using FFT Channel Estimator 59

3.6 Comparison of Simulated and Analytical Results for MSE of Equalizer Output with Channel Estimation Errors using Wiener Channel Estimator 59

3.7 Evaluation of BER Estimation Approaches for an Uncoded QPSK System using FFT Channel Estimator with FET Pilots in WINNER C2 Channel (no mobility) . . . . . . . . . . . . .

3.8 Evaluation of BER Estimation Approaches for Uncoded QPSK SC Systems using Wiener Channel Estimator with FET Pilots in WINNER C2 Channel (no mobility) . . . . . . . . . . . . .

3.9 Evaluation of BER Estimation Approaches for Uncoded 16-QAM SC Systems using Wiener Channel Estimator with FET Pilots in WIN-

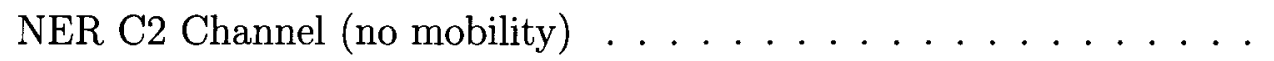

3.10 Comparison of BER and FER Performance using Different Simulation Approaches for a Coded QPSK System with FET Pilots in WINNER C2 Channel (Parameter Set 2-B) . . . . . . . . . . . 
4.1 Graphical Illustration of Two Variants of FDM Pilots: Frequency Expanding Technique (FET) and Frequency Domain Superimposed Pilot Technique $($ FDSPT) $\ldots \ldots \ldots \ldots$

4.2 Magnitude of I- and Q-Phase of SC Signal with FDSPT with and without IFDMA Training Signal ( $\alpha=0, \eta=3.125 \%$, QPSK) . . . . .

4.3 Magnitude of I- and Q-Phase of SC Signal for FET with and without IFDMA Pilot Signal $(\eta=3.125 \%$, QPSK $) \ldots \ldots . \ldots 71$

4.4 Comparison of CCDF of Magnitude of $x(n)$ for SC Modulated Signals with FDM Pilots $($ Parameter Set 1) . . . . . . . . . . . 72

4.5 Power Spectra of Signal Emerging from a Rapp Model $\left(p_{o}=2\right)$ Power Amplifier, with Backoffs Required to Satisfy a Spectral Mask (Parameter $\operatorname{Set} 1) \ldots \ldots \ldots \ldots \ldots \ldots$

4.6 BER Performance of FDSPT and FET for Uncoded System using frequency domain LMMSE Equalizer with Known CIR (Parameter Set

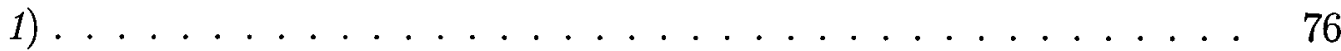

4.7 BER Performance of FDSPT and FET for Uncoded System using frequency domain IBSDFE Equalizer with Known CIR (Parameter Set

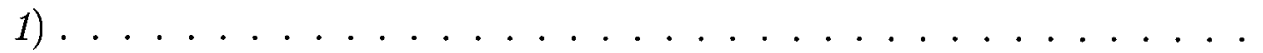

4.8 Block Diagram of the Proposed Decision-directed Interference Cancellation Technique for SC Signals with FDSPT Pilots . . . . . . 78

4.9 BER Performance of FDSPT for Uncoded System using Linear MMSE Equalizer with Iterative Decision-directed Interference Cancellation and known CSI (Parameter Set 2-B) . . . . . . . . . . . 80

4.10 BER Performance of FDSPT $(\alpha=0)$ for Uncoded System using IBSDFE Equalizer with Known CSI (IDIC: iterative decision-directed interference cancellation), Parameter Set 2-B . . . . . . . . 82

4.11 BER Performance of FDM Pilots for Coded System using frequency domain LMMSE Equalizer with Channel Estimation (Parameter Set 1) 
5.1 Block Diagram of the Proposed Frequency Domain Iterative DecisionDirected Channel Estimator for SC Systems with or without Decoder $\quad 89$

5.2 Effects of the Threshold Values for IDDCE (Parameter Set 2-B) . . 94

5.3 MSE Performance of Different Non-Iterative Channel Estimators for Parameter Set $2-A \ldots \ldots \ldots \ldots \ldots$

5.4 MSE Performance of IDDCE using FET Pilots for Parameter Set 2-B 97

5.5 FER Performance of FDM Pilots for Coded System using IBSDFE Equalizer with Non-Iterative Channel Estimations (Parameter Set 2-A) 98

5.6 FER Performance of FDM Pilots for Coded System using IBSDFE Equalizer with Non-Iterative Channel Estimations (Parameter Set 2-A) 98

5.7 FER Performance of Coded SC Systems with FDM Pilots using the Proposed IDDCE and IBSDFE Equalizer (Parameter Set 2-B) $\ldots 100$

5.8 FER Performance of Coded OFDM Systems with FDM Pilots using the IDDCE Proposed in [56] and Linear Equalizer (Parameter Set 2-B) 100

5.9 FER Performance of FDSPT Pilots for Coded SC Systems using IBSDFE Equalizer with Iterative Decision-directed Interference Cancellation, with Channel Estimation (Parameter Set 2-B) . . . . . . 101

5.10 FER Performance of FDM Pilots for Coded System using IBSDFE Equalizer with Non-Iterative Channel Estimation (Parameter Set 2-A) 102

5.11 FER Performance of FDM Pilots for Coded System using IBSDFE Equalizer with Iterative Channel Estimations (Parameter Set Q-A) . . 102

5.12 FER Performance of FDM Pilots for Coded OFDM Systems using Linear Equalizer and Non-iteratve Channel Estimator (Parameter Set

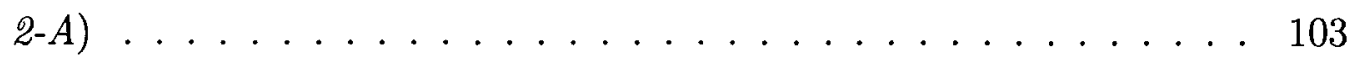

5.13 FER Performance of FDM Pilots for Coded OFDM Systems using Linear Equalizer and Iterative Channel Estimator (Parameter Set 2-A) 104 
5.14 Power Spectra of Signal Emerging from a Rapp Model $p_{o}=2$ Power Amplifier, with Back-offs Required to Satisfy a Spectral Mask, $\rho=$ Power Back-off . . . . . . . . . . . . . . . . . . . . 106

6.1 Resource Allocations of a Chunk Size System for a Frame . . . . . . . 111

6.2 Allocation of Chunks for Five Active Users . . . . . . . . . . . . 113

6.3 F1T2 Pilot Arrangement for IFDMA Systems . . . . . . . . . 116

6.4 Pilot Arrangements for B-IFDMA Systems . . . . . . . . . . . 117

6.5 Pilot Arrangement for Multiple Half Duplex Frames . . . . . . . . . 119

6.6 MSE Performance of Different Channel Estimators for Chunk Size Systems using Parameters Set 2 and the 5 User Chunk System Shown in

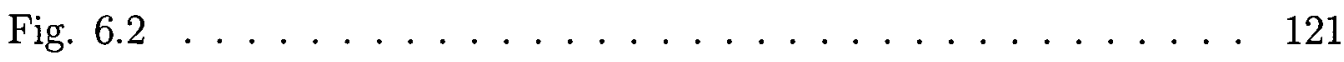

6.7 Average Mean Square Non-iterative Channel Estimation Error for IFDMA for Estimation over Several Frames in Half and Full Duplex Cases . . 123

6.8 Average Mean Square Iterative Channel Estimation $(\lambda=0.3)$ Error for IFDMA for Estimation over Several Frames in Half and Full Duplex Cases . . . . . . . . . . . . . . . . . . . . . . . . . . 124

6.9 Average Mean Square Non-iterative Channel Estimation Error for Full Bandwidth Systems for Estimation over Several Frames in Half and Full Duplex Cases . . . . . . . . . . . . . . . . . . . . . . . . . 124

6.10 Average Mean Square Iterative Channel Estimation $(\lambda=0.3)$ Error for Full Bandwidth Systems for Estimation over Several Frames in Half and Full Duplex Cases . . . . . . . . . . . . . . . . . 125

6.11 Effects of Vehicle Speed on Averaged MSE of Non-iterative and Iterative Channel Estimation for B-IFDMA System over $Q=12$ Half Duplex Frames, $E_{b} / N_{o}=12 \mathrm{~dB}$ and Pilot Arrangement $=$ F2T1 $(\mathrm{Pa}-$ rameters $\operatorname{Set} 2-B) \ldots \ldots \ldots \ldots \ldots \ldots \ldots \ldots$ 
6.12 FER Performance of FDM Pilots for Coded Systems using Frequency Domain IBSDFE Equalizer with Non-Iterative Channel Estimations over One Frame for Chunk Size Systems (Parameters Set 2-A) . . . .

6.13 FER Performance of FDM Pilots for Coded Systems using Frequency Domain IBSDFE Equalizer with Iterative Channel Estimations over One Frame for Chunk Size Systems (Parameters Set $2-A)$. . . . . 127

6.14 FER for IFDMA with FET Pilot Arrangement F1T2, with Non-iterative (W) and Iterative (ICE) Channel Estimation over Multiple Half Duplex Frames (Parameters Set 2-B) . . . . . . . . . . . . . 128

6.15 FER for B-IFDMA with FET Pilot Arrangement F1T1, with Noniterative (W) and Iterative (ICE) Channel Estimation over Multiple Half Duplex Frames (Parameters Set 2-B) . . . . . . . . . . . . . 129

6.16 FER for B-IFDMA with FET Pilot Arrangement F2T1, with Noniterative (W) and Iterative (ICE) Channel Estimation over Multiple Half Duplex Frames (Parameters Set 2-B) . . . . . . . . . . . 130

6.17 FER for 2B-IFDMA with FET Pilot Arrangement F2T2, with Noniterative (W) and Iterative (ICE) Channel Estimation over Multiple Half Duplex Frames (Parameters Set 2-B) . . . . . . . . . . . . 131

6.18 FER for Full Bandwidth with FET Pilot Arrangement F256T2, with Non-iterative (W) and Iterative (ICE) Channel Estimation over Multiple Half Duplex Frames (Parameters Set 2-B) . . . . . . . . . . . 131

7.1 Block Diagram of PAPR Reduction using Pilot Sequences (a: data sequences; $\mathbf{p}^{(k)}: k$ th pilot sequences; $N_{s}$ : number of pilot sequences to

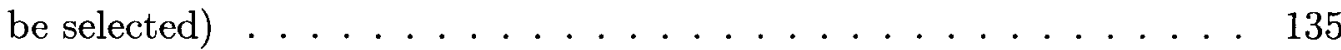

7.2 CCDF of Magnitude of OFDM and SC Signals with Parameters: $M=$ $64, I=4, N=8$, QPSK, FET, Selection Rule = PAPR, W-H and CS-Chu Pilot Sequences . . . . . . . . . . . . . . . 139

xvii 
7.3 CCDF of Magnitude of OFDM and SC Signals with Parameters: $M=$ $512, I=4, N=128$, QPSK, FET, Selection Rule $=$ PAPR, W-H and CS-Chu Pilot Sequences . . . . . . . . . . . . . . .

7.4 CCDF of Magnitude of OFDM and SC Signals with Parameters: $M=$ $416, I=4, N=104$, QPSK, FET, CS-Chu Pilot Sequences, Selection Rule $=$ PAPR $\ldots \ldots \ldots \ldots \ldots \ldots \ldots \ldots$

7.5 CCDF of Magnitude of SC Signals with Parameters: $M=416, I=$ $4, N=104,16-\mathrm{QAM}, \mathrm{FET}$, CS-Chu Sequences . . . . . . . . 141

7.6 AM/AM Curves of Rapp Model with Different Values of $p_{0} \ldots \ldots$.

7.7 PSD of SC and OFDM Signals with Parameters: $M=64, N=8, I=$ 4, QPSK, FET, CS-Chu Sequences, TD Window Rolloff Factor $=5.5 \%$, PAPR Selection Rule, $p_{o}=10$ and $\beta=5 \mathrm{~dB} \ldots \ldots \ldots . \ldots 143$

7.8 PSD of SC and OFDM Signals with Parameters: $M=416, N=$ 104, $I=4$, QPSK, FET, CS-Chu Sequences, TD Window Rolloff Factor $=5.5 \%$, PAPR Selection Rule, $p_{o}=10$ and $\beta=7 \mathrm{~dB} \ldots \ldots . .143$

7.9 PSD of SC and OFDM Signals with Parameters: $M=416, N=$ 104, $I=4$, QPSK, FET, CS-Chu Sequences, TD Window Rolloff Factor $=5.5 \%$, PAPR Selection Rule, $p_{o}=2$ and $\beta=7 \mathrm{~dB} \ldots \ldots$

7.10 PSD of SC Signals with Different Selection Rules $(M=416, N=$ $104, I=4,16$-QAM, FET, CS-Chu Sequences, TD Window Rolloff Factor $=5.5 \%, N s=32, p_{o}=10$ and $\left.\beta=7 \mathrm{~dB}\right) \ldots \ldots \ldots$

B.1 Effect of the Number of Users $P_{u}$ on Avg. $S N R$ for IFDMA and DSCDMA Systems in SUI5 Channel $\left(E s / N o=20[\mathrm{~dB}], K_{s}=64, M=16\right) 158$

B.2 Effect of $K_{s}$ on Avg. $S N R$ for IFDMA and DS-CDMA Systems in SUI5 Channel $\left(E s / N o=20[\mathrm{~dB}], P_{u}=1\right) \ldots \ldots \ldots \ldots$

B.3 Effect of $E s / N o$ on Avg. $S N R$ for IFDMA and DS-CDMA Systems in SUI5 Channel . . . . . . . . . . . . . . . . . . . 160

xviii 
B.4 Effect of $N_{A}$ on Avg. $S N R$ for IFDMA and DS-CDMA Systems in SUI5 Channel $\left(E s / N o=20[\mathrm{~dB}], P_{u}=1\right) \ldots \ldots 161$

B.5 Comparison of the Theoretical Results with the Simulation Results for IFDMA System with Constant Frequency Offset in AWGN Channel $\left(E s / N o=20[\mathrm{~dB}], P_{u}=1\right) \ldots \ldots \ldots \ldots \ldots$

B.6 Illustration of the Two-Sided 3-dB Bandwidth of the Lorentzian PSD 166

B.7 Performance Degradation for IFDMA System with Wiener Phase Noise in AWGN Channel $\left(E s / N o=20[\mathrm{~dB}], P_{u}=1\right) \ldots \ldots 168$

B.8 Average SNR for IFDMA and DS-CDMA System with Constant Frequency Offset in AWGN Channel $\left(E s / N_{O}=20[\mathrm{~dB}], N_{A}=1\right) \ldots 169$

B.9 Average SNR for IFDMA and DS-CDMA System with Constant Frequency Offset in SUI5 Channel $\left(E s / N o=20[\mathrm{~dB}], N_{A}=1\right) \ldots 170$

B.10 Average SNR for IFDMA and DS-CDMA System with Frequency Offset Modeled as an Uncorrelated Gaussian R.V. in AWGN Channel $\left(E s / N o=20[\mathrm{~dB}], N_{A}=1\right) \ldots \ldots \ldots \ldots \ldots$

B.11 Average SNR for IFDMA and DS-CDMA System with Frequency Offset Modeled as an Uncorrelated Gaussian R.V. in SUI5 Channel (Es/No= $\left.20[\mathrm{~dB}], N_{A}=1\right) \ldots \ldots \ldots \ldots \ldots \ldots \ldots$

B.12 Average SNR for IFDMA and DS-CDMA System with Wiener Phase Noise in AWGN Channel $\left(E s / N o=20[\mathrm{~dB}], N_{A}=1\right) \ldots 172$

B.13 Average SNR for IFDMA and DS-CDMA System with Wiener Phase Noise in SUI5 Channel $\left(E s / N o=20[\mathrm{~dB}], N_{A}=1\right) \ldots \ldots 173$

B.14 Average SNR for IFDMA and DS-CDMA System with Variance of Phase Noise Modeled as an Uncorrelated Gaussian R.V. in AWGN Channel $\left(E s / N o=20[\mathrm{~dB}], N_{A}=1\right) \ldots \ldots \ldots \ldots$

B.15 Average SNR for IFDMA and DS-CDMA System with Variance of Phase Noise Modeled as an Uncorrelated Gaussian R.V. in SUI5 Chan$\operatorname{nel}\left(E s / N o=20[\mathrm{~dB}], N_{A}=1\right) \ldots \ldots \ldots \ldots \ldots \ldots$

xix 


\section{List of Tables}

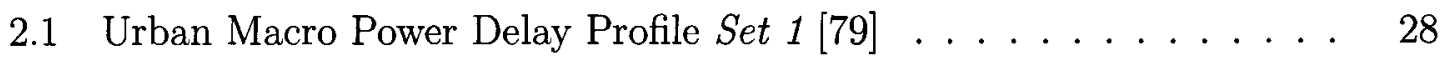

2.2 Simulation System Parameters Set 1 [79] . . . . . . . . . . . . 29

2.3 Urban Macro Power Delay Profile Set $2[67]$. . . . . . . . . . . . . . 29

2.4 Simulation System Parameters Set 2 . . . . . . . . . . . . 29

2.5 Summary of Challenges of Channel Estimation for Uplink SC Systems in Time-varying Frequency Selective Fading Channel . . . . . . . . 46

4.1 Comparison of Different Pilot Arrangement Schemes . . . . . . . . . 84

5.1 Different Scenarios of Mis-Matched Parameter for Generating Wiener Filter Taps . . . . . . . . . . . . . . . . . 101

5.2 Comparison of OFDM and SC Performance and Overhead for TDM and FDM Pilot Schemes (SNR at FER $=10^{-2}$ is obtained from Fig. 5.10 to Fig. 5.13 and IBSDFE equalizer is used for SC systems) . . 105

5.3 Comparison of Required Power Backoffs for OFDM and SC Systems . 105

B.1 Simulation Parameters for Evaluation of IFDMA and CDMA Systems 158 


\section{List of Abbreviations}

1D-FIR 1-Dimensional Finite Impulse Response

2D 2-dimensional

APP A Posteriori Probability

AWGN Additive White Gaussian Noise

BER Bit Error Rate

CDMA Code Division Multiple Access

CEE Channel Estimation Errors

CFR Channel Frequency Response

CIR Channel Impulse Response

CLT Central Limit Theorem

CP Cyclic Prefix

CSI Channel State Information

DFT Discrete Fourier Transform

FDM Frequency Domain Multiplexed

FDOSS Frequency Domain Orthogonal Signature

FDSPT Frequency Domain Superimposed Pilot Technique

FER Frame Error Rate

FET Frequency Expanding Technique

FFT Fast Fourier Transform

GMC Generalized Multicarrier

HPA High Power Amplifier

IBSDFE Iterative Block Soft Decision Feedback Equalizer

xxi 
IDDCE Iterative Decision-Directed Channel Estimation

IDFT Inverse Discrete Fourier Transform

IFDMA Interleaved Frequency Division Multiple Access

IFFT Inverse Fast Fourier Transform

LAN Local Area Network

LLR Log-Likelihood Ratio

LLSLF Linear Least Square Line Fitting

LMMSE Linear Minimum Mean Square Error

LS Least Square

MAP Maximum A Posteriori

MMSE Minimum Mean Square Error

OFDM Orthogonal Frequency Division Multiplexing

PACE Pilot-Aided Channel Estimation

PAPR Peak-to-Average Power Ratio

$\mathrm{SC} \quad$ Single-Carrier

SSE Sum of Square Error

SUI-5 Stanford University Interim Channel Model \#5

TDM Time Domain Multiplexed

W-H Walsh-Hadamard

WINNER Wireless World Initiative New Radio 


\section{List of Symbols}

page where

symbol

$\underline{\text { Symbol }}$

$\underline{\text { Explanation }}$

is defined

$A_{\ell}$

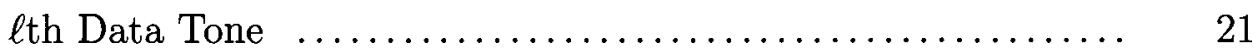

$\alpha$

Scaling Factor for the Data Tones at the Pilot Locations $\ldots . . .67$

$a_{m}$

$m$ th Data Symbol $\ldots \ldots \ldots \ldots \ldots \ldots \ldots \ldots \ldots \ldots \ldots \ldots \ldots$

$\beta$

Scaling Factor for the Pilot Tones $\ldots \ldots \ldots \ldots \ldots \ldots \ldots .67$

$c_{k}$

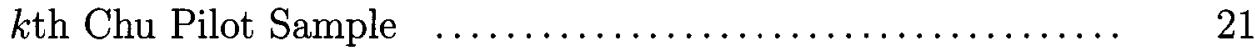

$C_{\ell} \quad \ell$ th Frequency Domain Equalizer Tap $\ldots \ldots \ldots \ldots \ldots \ldots \ldots$

$\Delta F \quad$ Subcarrier Spacings $\ldots \ldots \ldots \ldots \ldots \ldots \ldots \ldots \ldots \ldots \ldots \ldots \ldots \ldots \ldots \ldots \ldots \ldots \ldots$

$\Delta F_{c} \quad$ Carrier Offset $\ldots \ldots \ldots \ldots \ldots \ldots \ldots \ldots \ldots \ldots \ldots \ldots \ldots \ldots \ldots \ldots \ldots \ldots \ldots$

$\Delta f \quad$ Normalized Constant Carrier Frequency Offset $\ldots \ldots \ldots \ldots \ldots$

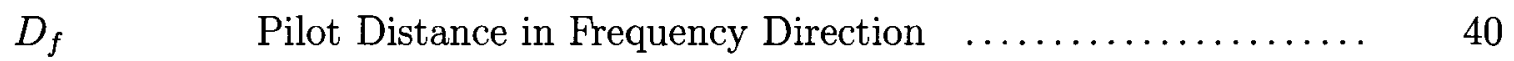

$D_{t} \quad$ Pilot Distance in Time Direction $\ldots \ldots \ldots \ldots \ldots \ldots \ldots \ldots \ldots \ldots$

$\frac{E_{b}}{N_{0}} \quad$ Ratio of Energy per Data Bit to the Noise Power

in the Signal Bandwidth $\ldots \ldots \ldots \ldots \ldots \ldots \ldots \ldots \ldots \ldots . . . \ldots \ldots$

$\frac{E_{s}}{N_{0}} \quad$ Ratio of Energy per Coded Data Symbol to the Noise

Power in the Signal Bandwidth $\ldots \ldots \ldots \ldots \ldots \ldots \ldots \ldots \ldots .28$

$f_{\text {Dmax }} \quad$ One-Sided Maximum Doppler Spread $\ldots \ldots \ldots \ldots \ldots \ldots \ldots \ldots$

$\hat{H}_{k, \ell} \quad$ LS Estimate of Channel Frequency Response at the $\ell$ th Frequency in the $k$ th Block within a Frame $\ldots \ldots \ldots \ldots \ldots .38$

$H_{k, \ell} \quad$ Channel Frequency Response at the $\ell$ th Frequency

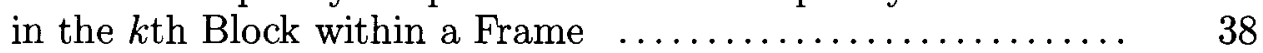

xxiii 


\begin{tabular}{|c|c|}
\hline$H_{\ell}$ & $\ell$ th Frequency of Channel Frequency Response $\ldots \ldots \ldots \ldots$ \\
\hline$I$ & Upsampling Factor \\
\hline$I_{f}$ & Number of the Frequency Direction Filter Taps \\
\hline$I_{t}$ & Number of Time Direction Filter Taps \\
\hline$K_{s}$ & Spreading Factor for a CDMA System \\
\hline$L$ & Number of Samples per Block $\ldots . . .$. \\
\hline$\Lambda$ & Constellation Size \\
\hline$\lambda$ & Threshold Value of the Proposed IDDCE Estimator \\
\hline$L_{c p}$ & Number of Cyclic Prefix Samples ... \\
\hline$M$ & Number of Data Symbols in a Block ... \\
\hline$M_{c}$ & Number of Chips per Block for a CDMA System $\ldots .$. \\
\hline$N$ & Number of Pilots per Block . \\
\hline$N_{A}$ & Number of Receiving Antenna Elements in a CDMA System . \\
\hline$N_{B}$ & Number of Blocks per Frame $\ldots .$. \\
\hline$N_{b}$ & Number of Blocks with Pilots $\ldots .$. \\
\hline$N_{b l k}$ & Number of Blocks of Subcarriers per OFDM Symbol \\
\hline$N_{c h}$ & Number of Discrete Time Channel Samples \\
\hline$N_{d}$ & Number of Data Symbols per Block for a CDMA System \\
\hline$N_{d a t a}$ & Number of Data Symbols per Coded Frame \\
\hline$N_{f}$ & Number of Consecutive Subcarriers per Block in a Chunk $\ldots$. \\
\hline$N_{F}$ & Subcarrier Separation between Chunks $\ldots$ \\
\hline$N_{F F T}$ & FFT Size in a Block \\
\hline$N_{p}$ & Number of Pilots per Coded Frame \\
\hline$N_{t}$ & Number of Consecutive Blocks in a Chunk. \\
\hline$\Omega$ & $\begin{array}{l}\text { The set contains the frequency locations of the pilots } \\
\text { within the } k \text { th block } \ldots \ldots \ldots \ldots \ldots \ldots \ldots \ldots \ldots \ldots \ldots \ldots \ldots\end{array}$ \\
\hline$P_{k, \ell}$ & $\begin{array}{l}\text { Pilot Frequency Samples at the } \ell \text { th Frequency } \\
\text { in the } k \text { th Block within a Frame } \ldots \ldots \ldots \ldots .\end{array}$ \\
\hline$P_{\ell}$ & 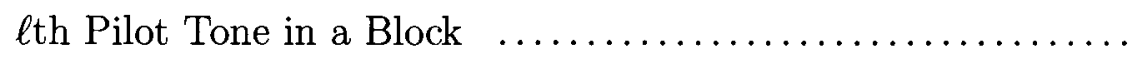 \\
\hline
\end{tabular}

xxiv 


\begin{tabular}{|c|c|}
\hline$\Psi$ & $\begin{array}{l}\text { The set contains the index of the blocks with pilots } \\
\text { within a frame } \ldots \ldots \ldots \ldots \ldots \ldots \ldots \ldots \ldots \ldots \ldots \ldots \ldots \ldots \ldots \ldots \ldots\end{array}$ \\
\hline$P_{u}$ & Number of Active Users in a CDMA System ... \\
\hline$Q$ & Number of Active OFDM Symbols per Frame $\ldots \ldots$ \\
\hline$r$ & Channel Coding Rate \\
\hline$\rho$ & Power Backoff of the Amplifier in $\mathrm{dB}$ \\
\hline$\sigma^{2}$ & Variance of White Noise $\ldots .$. \\
\hline$S_{p}$ & Pilot Boost Factor \\
\hline$\tau_{\max }$ & One-Sided Maximum Delay Spread \\
\hline$T_{B}$ & Time Duration of the Block including CP \\
\hline$T_{s}$ & Symbol Time for SC or Sample Time for OFDM \\
\hline$V_{\ell}$ & frequency domain noise sample at frequency $\ell$ \\
\hline$X_{\ell}$ & eth Data or Pilot Tones \\
\hline$Y_{k, \ell}$ & $\begin{array}{l}\text { Frequency Domain Received Signal at the } \ell \text { th Frequency } \\
\text { in the } k \text { th Block within a Frame } \ldots \ldots \ldots \ldots \ldots \ldots \ldots \ldots \ldots\end{array}$ \\
\hline$Y_{\ell}$ & lth Received Frequency Sample in a Block \\
\hline
\end{tabular}




\section{Chapter 1}

\section{Introduction}

Future generation (4G) wireless communication systems will support a wide variety of user-oriented services and applications in various radio environments, such as those from short range to wide area, from low to high data rate or from low to high user mobility. The vision is to communicate anywhere to anyone at anytime. To achieve this goal requires a highly flexible and scalable system with respect to user bandwidth requirements. 4G systems will be all-IP (Internet Protocol) based, providing not only voice but also data and multimedia transmission at low transmission cost [1]. In order to address high flexibility and scalability with respect to data rates and radio environments, the future converged wireless world requires in the long-term perspective a ubiquitous radio system instead of disparate systems for different purposes (cellular, wireless LAN, short-range access, etc.) [2]. Fig. 1.1 shows the concept of a ubiquitous radio system, proposed by Wireless World Initiative New Radio (WINNER) ${ }^{1}$. The short range applications are characterized by low mobility but high data rates. On the other hand, wide area applications are characterized by relatively lower data rates but higher mobility. The data rate can range from $1 \mathrm{Mbps}$ in the wide area scenarios to $1 \mathrm{Gbps}$ in the short range scenarios, while the supported mobility can be greater than $100 \mathrm{~km} / \mathrm{h}$.

\footnotetext{
${ }^{1}$ WINNER is a consortium of 41 partners co-ordinated by Nokia Siemens Networks working towards enhancing the performance of mobile communication systems. The improvements of radio transmission to be explored by WINNER are crucial for enabling new mobile services and applications anytime and anywhere. The challenge is to make mobile communication systems more adaptable to user needs [3].
} 


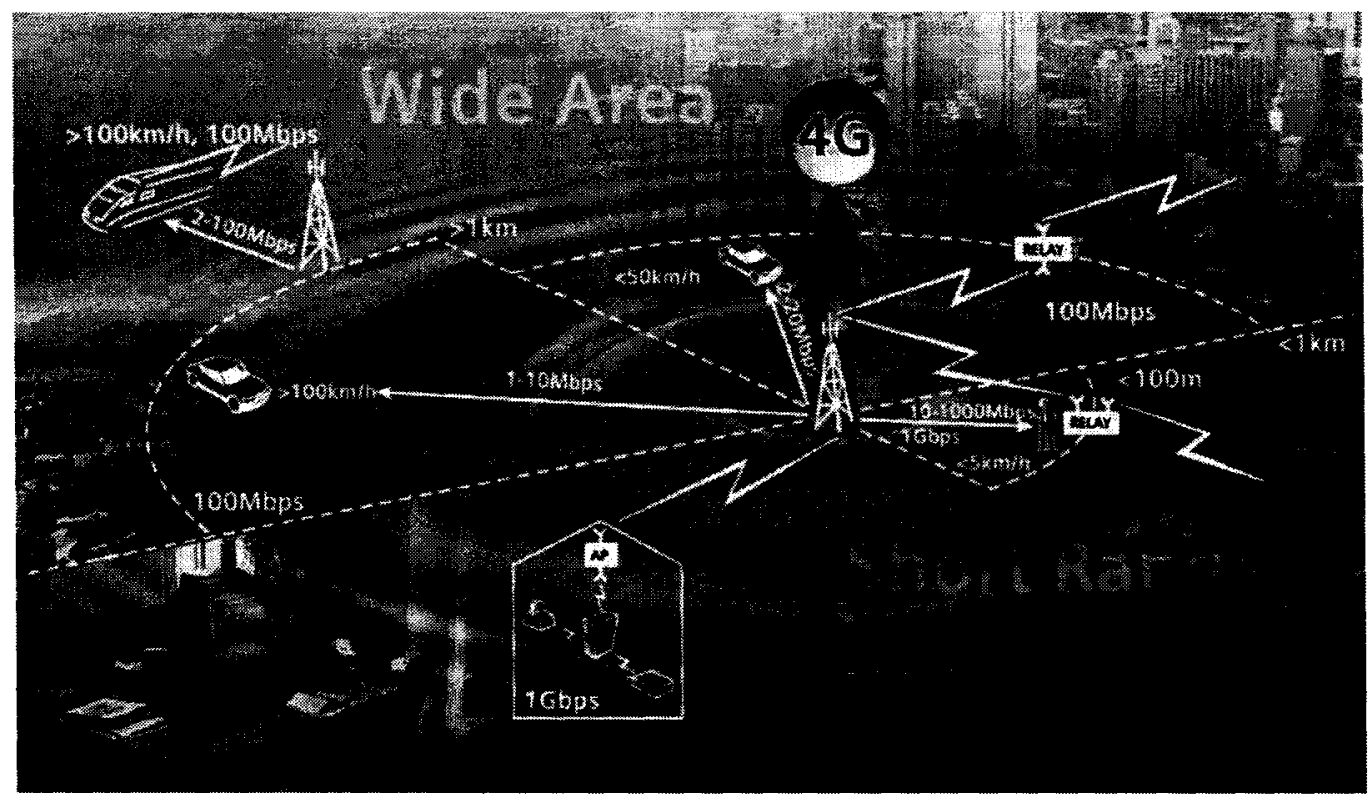

Figure 1.1: WINNER's View of A Ubiquitous 4G Radio System Concept

\subsection{Motivation}

With the flexibility and scalability requirements for $4 \mathrm{G}$ systems, there exists a challenge for designing a low cost user terminal, especially in the wide area, with high spectral efficiency and coverage, and ease of deployment for service providers. The choice of modulation and coding schemes can significantly influence performance and cost for a given range of data rates. $4 \mathrm{G}$ wireless systems will likely use flexible combinations of frequency domain block transmission methods such as orthogonal frequency division multiplexing (OFDM) and single carrier (SC) with frequency domain equalization [24,67]. For example, SC may be preferred for the uplink of cellular systems (in wide area) because of its low peak-to-average power ratio (PAPR) and the resulting power amplifier efficiency in the user terminal. SC with frequency domain equalization using computationally-efficient fast Fourier transform (FFT) and inverse FFT (IFFT) operations can be applied to SC modulation schemes, in which data symbols are serially modulated with a high symbol rate, for low implementation complexity. Moreover, the SC signal (and OFDM signal) can also be generated in the 
frequency domain using the generalized multicarrier (GMC) ${ }^{2}$ transmission technique $[4,21]$. Frequency domain signal generation facilitates flexible and efficient assignment of signals to available spectrum [21]. With the GMC approach, a SC signal can be viewed as an OFDM signal with discrete Fourier transform (DFT)-precoding, i.e. the DFT of the data symbols are used instead of the data symbols before the inverse DFT (IDFT) operation. Hence, SC systems generated in this way can also be called DFT-precoded OFDM systems. Generating SC signals in the frequency domain avoids conventional complex symbol-by-symbol filtering and affords an extra degree of spectrum flexibility.

A channel estimation scheme (including pilot arrangement) with low pilot overhead and low implementation complexity is desirable for an efficient coherent receiver. For SC systems, the pilot symbols are traditionally time domain multiplexed (TDM) within or in between the transmission blocks $[28,45,47,48]$. With the aid of the GMC transmission technique and the concept of interleaved frequency division multiple access (IFDMA) ${ }^{3}$ [78], it is also possible to use the in-band frequency domain multiplexed (FDM) pilots and frequency domain channel estimation for SC systems to take the advantage of flexible and efficient assignment of signals to available spectrum with reasonable implementation complexity.

\subsection{Objectives and Scope}

In this thesis, we mainly focus on developing and evaluating efficient frequency domain channel estimation and equalization techniques for future uplink SC wireless communication systems using TDM and FDM pilots, in terms of bit error rate (BER) and frame error rate (FER) performance, pilot overhead, implementation complexity and system flexibility (e.g., compatibility with an OFDM transceiver) in wide area

\footnotetext{
${ }^{2}$ Note that a more general class of signals, such as CDMA, OFDMA, MC-CDMA, IFDMA, can also be generated using the GMC technique [21]. In this thesis, we focus on SC and OFDM signals and collectively consider SC and OFDM systems as special cases of GMC systems.

${ }^{3}$ IFDMA has had many names, such as FDOSS (frequency domain orthogonal signature sequence) CDMA since the signature codes for the users are orthogonal in the frequency domain [77].
} 
environments. Moreover, we want to compare the performance of the SC and OFDM systems using FDM pilots. We assume the broadband channels are non-line-of-sight (NLOS) multipath time-varying fading channels. To achieve our goals, we have the following objectives:

- Analyze the effects of channel estimation errors on BER performance of a frequency domain linear minimum mean square error (MMSE) equalizer;

- Investigate the feasibility of using in-band FDM pilots for SC signals and the effects of SC signals with FDM pilots in terms of peak to average power ratio (PAPR);

- Develop and evaluate frequency domain equalization techniques for SC signals with FDM pilots;

- Design and evaluate frequency domain channel estimators for SC signals with FDM pilots in broadband time-varying frequency selective fading channels;

- Evaluate and compare the performance (BER and FER) of coded SC and OFDM systems with TDM and FDM pilots;

- Apply frequency domain channel estimation techniques, designed for full bandwidth systems, for chunk and sub-chunk size systems (for which each user occupies partial available signal bandwidth);

- Investigate the effectiveness of channel estimation over multiple half and full duplex frames in time-varying frequency selective fading channels;

- Evaluate and compare the performance for full bandwidth, chunk size and subchunk size systems using different channel estimation techniques;

- Determine if frequency domain channel estimation using FDM pilots is more suitable than that using TDM pilots for uplink SC for future generation wireless communication systems in wide area scenarios. 


\subsection{Thesis Contributions}

We have in this thesis made several contributions to the knowledge of the field of digital communications, which are summarized as follows:

1. The analysis of the effects of channel estimation errors, in the frequency domain, on the BER performance of uncoded SC systems using frequency domain linear MMSE equalizer. (Chapter 3)

- An analytical expression relating the time-average MSE of the frequency channel estimates to BER performance of uncoded SC systems. (Sec. 3.2)

- The development of a statistical model (Gaussian) for the error of the frequency channel estimates and the use of this model to estimate the BER performance for both coded and uncoded SC systems. (Sec. 3.3)

2. The development of frequency domain channel estimation and equalization techniques using in-band FDM pilots for full bandwidth SC systems. (Chapters 4 and 5)

- Design of two FDM pilot arrangement schemes for SC systems using the concept of IFDMA signal and GMC transmission technique. (Sec. 4.2)

- Analysis of SC signal with FDM pilots in terms of its PAPR and power spectra. (Sec. 4.2)

- Development of frequency domain channel equalization techniques for SC signals with in-band FDM pilots. (Sec. 4.3)

- Design of frequency domain iterative decision-directed interference cancellation technique for SC signals with FDSPT pilots. (Sec. 4.4)

- Design of frequency domain non-iterative hybrid channel estimation techniques for SC systems using FDM pilots in time-varying frequency selective fading channels. (Sec. 5.2) 
- Design and analysis of a low complexity frequency domain iterative decisiondirected channel estimation technique for SC systems using FDM pilots in time-varying frequency selective fading channels. (Sec. 5.3)

- Evaluation of MSE and FER performance for the proposed frequency domain channel estimation techniques. (Sec. 5.2 and 5.3)

3. Comparative study of the performance of different channel estimators using TDM and FDM pilots for full bandwidth SC and OFDM systems. (Chapter 5)

- Evaluation of FER performance for coded SC systems using FDM pilots with mis-matched channel knowledge for generation of the Wiener filter taps for channel estimation. (Sec. 5.4.3)

- Evaluation of power spectra of signal emerging from a high power amplifier (HPA) with input power backoffs required to satisfy a spectral mask. (Sec. 5.5)

- Comparison of the performance between TDM and FDM pilots with different channel estimators for SC and OFDM systems in terms of pilot and cyclic prefix (CP) overheads, required input power back-offs and FER. (Sec. 5.5)

4. The development of frequency domain channel estimation techniques for chunk size and sub-chunk size SC systems using FDM pilots. (Chapter 6)

- Design of FDM pilots for chunk size and sub-chunk size SC systems. (Sec. 6.2)

- Application of non-iterative and iterative frequency domain channel estimation techniques for chunk and sub-chunk size systems. (Sec. 6.3)

- Design and evaluation of channel estimation techniques over multiple full and half duplex frames for chunk, sub-chunk and full bandwidth systems. (Sec. 6.4 and 6.5) 
- Evaluation of MSE and FER performance for a coded chunk and sub-chunk size system in time-varying frequency selective fading channels. (Sec. 6.5)

5. The design and analysis of PAPR reduction techniques for SC and OFDM signals using a set of FDM pilot sequences. (Chapter 7)

- Investigation of the feasibility of PAPR reduction for SC and OFDM signals using Walsh-Hadamard sequences for large block size. (Sec. 7.3)

- Design and analysis of PAPR reduction for SC and OFDM signals using cyclic shifted Chu sequences [112] for small and large block size. (Sec. 7.3)

- Comparison of the performance of using the minimum PAPR [109] and sum of square error selection rule [110]. (Sec. 7.4)

- Evaluation of the effects on out-of-band distortion after HPA for SC and OFDM signals with PAPR reduction using Chu pilot sequences. (Sec. 7.5)

6. Evaluation and comparative study of the performance between direct sequence (DS)-CDMA and IFDMA systems in the presence of carrier frequency offset and Wiener phase noise. (Appendix B) ${ }^{4}$

- Performance comparison between DS-CDMA and IFDMA systems without frequency offset and phase noise. (Sec. B.2)

- Analytical results for single-user IFDMA systems with constant carrier frequency offsets and Wiener phase noise. (Sec. B.3)

- Comparison of multiuser DS-CDMA and IFDMA systems with frequency offset and phase noise. (Sec. B.4)

Some of the contributions have been published in several papers, while others are submitted for publication. The published and submitted contributions are as follows:

\footnotetext{
${ }^{4}$ Note that we put this contribution in the appendix for the coherence of the thesis. Its main connection with the rest of the thesis is through IFDMA, which is used to generate frequency domain multiplexed pilots.
} 
1. Channel Estimation for SC-FDE Systems Using Frequency Domain Multiplexed Pilots (presented in IEEE VTC'06 Fall) [6]

2. Frequency Domain Channel Estimation for Single-Carrier Uplink in Time-Varying Channels (presented in WWRF 16th Meeting) [7]

3. A Low Complexity Frequency Domain Iterative Decision-Directed Channel Estimation Technique for Single-Carrier Systems (presented in IEEE VTC'O7 Spring) [8]

4. Design of Time and Frequency Domain Pilots for Generalized Multicarrier Systems (presented in IEEE ICC'07) [9]

5. PAPR Reduction using Frequency Domain Multiplexed Pilot Sequences (presented in IEEE WCNC'O7) [10]

6. Channel Estimation for Sub-Chunk-based DFT-precoded OFDM Systems (presented in WWRF 18th Meeting) [11]

7. On the Impact of Phase Noise and Frequency Offsets in Block Transmission CDMA Schemes (presented in 1st International Symposium on Wireless Communication Systems 2004) [12]

8. A Multiple Access Scheme for the Uplink of Broadband Wireless Systems (presented in IEEE GLOBECOM'04) [13]

9. Carrier Synchronization Requirements for CDMA Systems with FrequencyDomain Orthogonal Signature Sequence (presented in IEEE ISSTA'04) [14]

10. Iterative Frequency Domain Channel Estimation for DFT-precoded OFDM Systems using In-band Pilots (submitted to IEEE Journal on Selected Areas in Commun. in 2007) [17] 
11. Power Backoff Reduction Techniques for Generalized Multicarrier Waveforms (to be presented in EUSIPCO Conference in Sept. 2007) [18]

12. Power Backoff Reduction Tehcniques for Generalized Multicarrier Waveforms (submitted to EURASIP Journal on Wireless Commun. and Networking in 2007) [19]

The idea of frequency domain channel estimation using the proposed in-band FDM pilots for SC systems is published in $[6,7,17]$. The low complexity frequency domain iterative decision-directed channel estimation technique for SC systems with FDM pilots is presented in the accepted paper [8] and the submitted paper [17]. The results for the comparative study of the performance of frequency domain channel estimators using TDM and FDM pilots for full bandwidth SC and OFDM systems are published in [7] and accepted to appear in [9]. The design and analysis of PAPR reduction techniques for $\mathrm{SC}$ and OFDM signals using a set of FDM pilot sequences is described in the presented paper [10] and the submitted papers [18] and [19]. Some of the results from the performance comparison between DS-CDMA and IFDMA system in the presence of frequency offset and phase noise are published in [12]. The use of IFDMA systems for uplink broadband wireless system is discussed in [13] and the synchronization requirements for IFDMA are addressed in [14].

\subsection{Thesis Overview}

Our main focus is on frequency domain channel estimation and equalization techniques for uplink SC systems with FDM pilots in time-varying frequency selective fading channels, while other topics are necessary to support the understanding of our proposed channel estimation and equalization techniques, as shown in Fig. 1.2. For example, we also consider channel estimation technique for OFDM systems in order to compare with our proposed channel estimation techniques using FDM pilots.

Chapter 2 provides necessary background and related literature review. A brief 


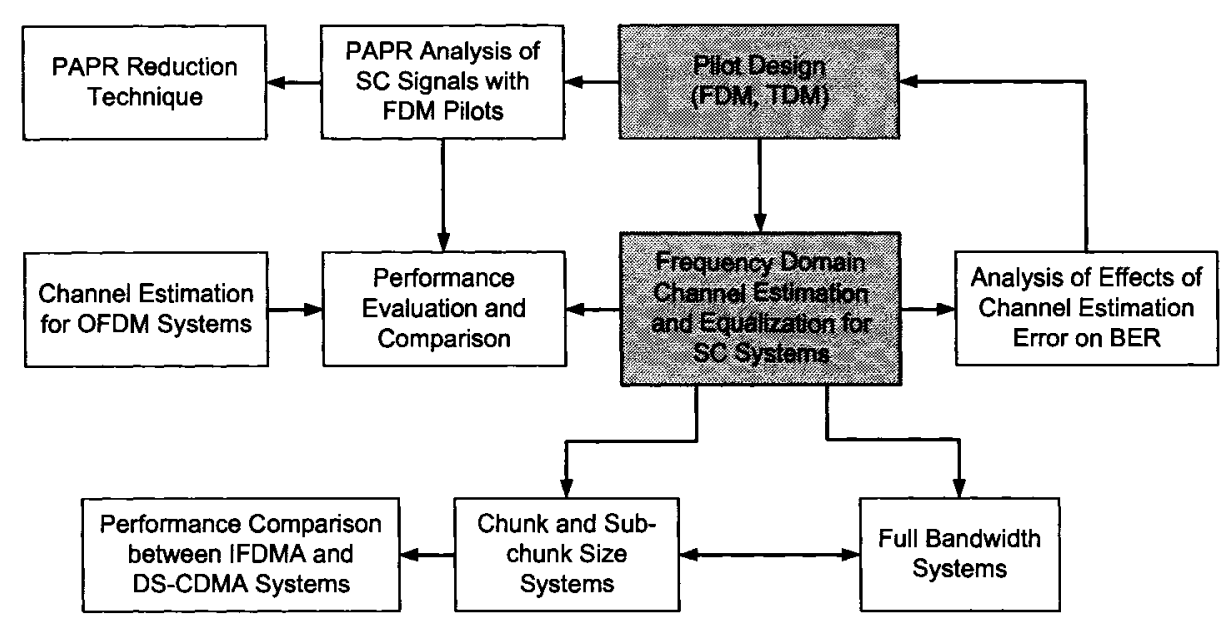

Figure 1.2: Relation of various Topics Considered in This Thesis

description of a general GMC transmitter and receiver structure is provided first. A summary of the comparison between SC and OFDM systems is then given, followed by the system descriptions, including simulation parameters to be used later in the thesis. Brief descriptions of the frequency domain non-iterative and iterative equalization techniques for SC systems are included. We then review the channel estimation techniques for SC and OFDM systems in time-varying frequency selective fading channels. Finally, we include the challenges of designing channel estimation techniques for uplink SC systems.

In Chapter 3, we analyze the effects of channel estimation errors, characterized by MSE, on BER performance of uncoded SC systems using frequency domain linear MMSE equalization. Analytical and semi-analytical approaches are used to obtain the estimation of BER performance given the time-averaged MSE of frequency channel estimates.

Chapter 4 provides detailed descriptions of the design of FDM pilots for SC systems and equalization techniques for the SC signals with FDM pilots. Two techniques of multiplexing FDM pilots for SC signals are introduced, namely the frequency expanding technique (FET) and the frequency domain superimposed pilot technique (FDSPT). The PAPR effects of multiplexing FDM pilots for SC signals using GMC 
transmission technique are addressed, followed by the frequency domain equalization of the SC signals with FDM pilots. Moreover, we propose an iterative decisiondirected interference cancellation technique for SC signals with FDSPT pilots.

In Chapter 5 we propose two non-iterative channel estimators and one iterative channel estimator using the proposed FDM pilots for full bandwidth systems in timevarying frequency selective fading channels. The effects of mis-matched knowledge of the maximum delay spread and the speed of the user terminal, when generating the Wiener interpolation filter taps, is also investigated. Moreover, we compare the performance of non-iterative and iterative channel estimators for SC systems using TDM and FDM pilot schemes in terms of pilot overhead, CP overhead, the required power backoff for the HPA and the FER. We also compare the performance of SC systems with that of coded OFDM systems with FDM pilots. The FER performance of SC systems with FDSPT pilots using the iterative block soft decision feedback equalizer (IBSDFE) with an iterative decision-directed interference cancellation technique is also included.

Chapter 6 discusses the application of the proposed frequency domain non-iterative and iterative channel estimations for chunk and sub-chunk size systems with FDM pilots. Similar to the full bandwidth case, the effects of mis-matched channel knowledge on the performance is included. We also consider channel estimation over multiple half or full duplex FDD (frequency division duplex) frames, which is shown to outperform channel estimation over one frame. We also look into the dependency on the effectiveness of multi-frame channel estimation.

Chapter 7 investigates the PAPR reduction method using Walsh-Hadamard and cyclic shifted Chu sequences for SC and OFDM signals with FDM pilots. We also investigate the applicability of the sum of square error selection rule for high order modulation of SC signals. The effects of out-of-band radiation for SC and OFDM signals with PAPR reduction using FDM pilot sequences after HPA are also included.

Chapter 8 provides the conclusions and some design recommendations as well 
as possible future research topics on channel estimation and equalization for GMC systems with FDM pilots.

Appendix A provides details on the derivation of some of the mathematical expressions presented in the main text of this thesis, while Appendix B provides the performance comparison between IFDMA and DS-CDMA systems with and without carrier frequency offsets and Wiener phase noise. 


\section{Chapter 2}

\section{Background and Literature Review}

This chapter provides necessary background and related literature reviews. A brief description of a general GMC transmitter and receiver structure is provided first. We then give a summary of the comparison between SC and OFDM systems in terms of BER performance and various practical implementation considerations, followed by the system descriptions, which include the structure of the GMC transmitter and receiver with FDM pilots. Brief descriptions of the frequency domain non-iterative linear MMSE (LMMSE) and frequency domain iterative block soft decision feedback equalization (IBSDFE) techniques for SC systems are included. We then review the channel estimation techniques for SC and OFDM systems in time-varying frequency selective fading channels. The review of the channel estimation techniques for OFDM systems is necessary as we want to consider applying channel estimation techniques typically used in OFDM systems for SC systems. Moreover, we apply one of the proposed FDM pilot schemes for OFDM systems and compare the performance of SC and OFDM systems with FDM pilots. Finally, we discuss some of the challenges of designing channel estimation techniques for uplink SC systems for future wireless systems. 


\subsection{Generalized Multicarrier (GMC) Systems}

Fig. 2.1 depicts a general GMC transmitter and receiver structure [21, Ch. 6]. In addition to SC and OFDM signals, a more general class of signals, such as DS-CDMA (direct sequence code division multiple access), MC-CDMA (multicarrier code division multiple access), IFDMA, can be generated using the GMC transmitter (shown in Fig. 2.1 (a)) by either including or bypassing the spreading matrix and/or the FFT matrix. For example, to generate SC signals, the spreading matrix is bypassed (or is an identity matrix) and the FFT matrix is included, while to generate OFDM signals, the FFT matrix is also bypassed. Before taking the inverse FFT (IFFT), the space-frequency selector matrix can be used to allocate data subcarriers to multiple transmitting antennas in a MIMO (multiple-input multiple output) or space-time code. The GMC receiver (shown in Fig. 2.1 (b)) reverses the processing of the transmitter. After removing cyclic prefix (CP), an FFT operation is used for frequency domain processing. The GMC architecture provides a general framework for air-interface technologies that are applicable to next generation wireless systems. It also suggests a modular signal processing architecture that can be exploited to allow modulation and multiple-access schemes to be changed dynamically in response to user requirements and the radio environment. All the basic forms of GMC systems offer an overall (transmitter and receiver) signal processing complexity that is approximately proportional to the logarithm of the maximum channel impulse response (CIR) length [21]. In this thesis, we consider SC and OFDM systems using the GMC approach and collectively refer to GMC systems. The detail structure of the GMC systems with FDM pilots will be given in Sec. 2.3.1.

\subsection{Single-Carrier versus OFDM Systems}

SC and OFDM are two favorite choices of modulation schemes for the uplink transmission for future broadband wireless communication system $[67,79]$, so there are 
(a) Transmitter

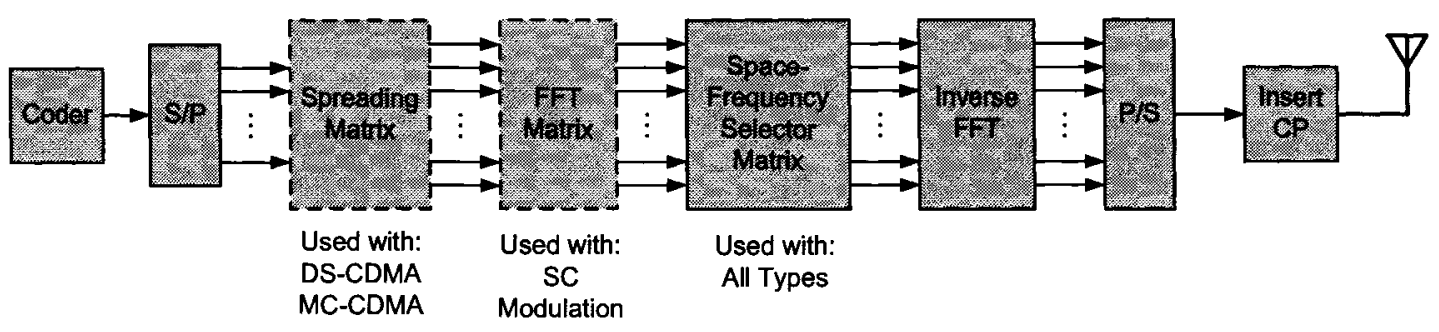

(b) Receiver

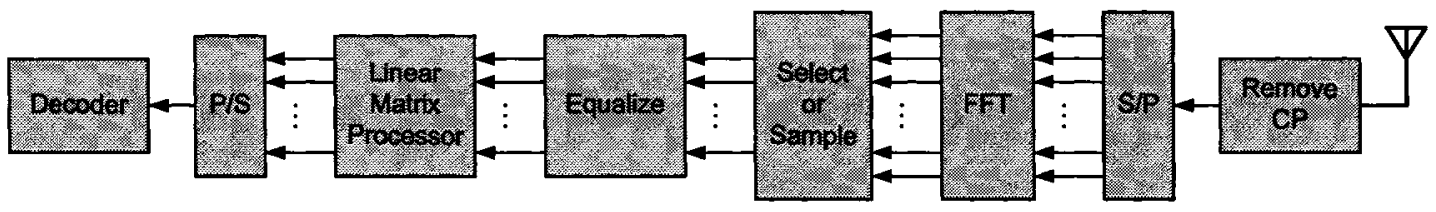

Figure 2.1: Generalized Multicarrier Transmitter and Receiver Structure [21, Ch. 6]

many comparisons (in terms of different system configurations) in the literature, such as $[21,24,25,28,33,79]$. We summarize the comparisons of the SC and OFDM systems in terms of the power amplifier efficiency, flexibility of spectrum allocations, BER performance, quantization effects and sensitivity to phase noise. Our motivation is to give an overview of the main advantages and disadvantages of choosing $\mathrm{SC}$ systems, which can be taken into account when designing pilot schemes and channel estimation techniques for uplink SC systems.

\subsubsection{Power Amplifier Efficiency}

Transmitter power amplifier efficiency is especially important for the user terminal, since the power amplifier usually constitutes a substantial portion of the cost of a wireless user terminal, and power amplifier cost rises very sharply with peak power rating. Peak output instantaneous power exceeding a power amplifier's linear range causes significant spectral re-growth, and BER degradation resulting from nonlinear distortion [23]. From the power amplifier efficiency point of view, the SC modulated system has the advantage of requiring smaller power backoff since it has lower PAPR than does the OFDM signal. Note that larger value of power backoff means more 
expensive power amplifiers. Consider block-wise transmission system with $M$ data symbols and $L$ samples per block. The discrete time samples of the SC modulated waveform, generated using GMC transmitter, can be written as [21]

$$
x_{S C}(n)=\sum_{m=0}^{M-1} a_{m} g\left(n-m \frac{L}{M}\right), \quad n=0,1, \ldots, L-1,
$$

where $a_{m}$ is the $m$ th data symbol and $g(k)=\frac{1}{L} e^{j \frac{\pi}{L}(L-1) k} \frac{\sin \left(\frac{\pi M}{L} k\right)}{\sin \left(\frac{\pi}{L} k\right)}$ is a bandlimited pulse waveform. The discrete time samples of the OFDM waveform can be written as the IDFT of the subcarriers $\left\{a_{\ell}\right\}_{\ell=0}^{M-1}[22]$,

$$
x_{O F D M}(n)=\frac{1}{L} \sum_{\ell=0}^{M-1} a_{\ell} e^{j \frac{2 \pi n \ell}{L}}, \quad n=0,1, \ldots, L-1 .
$$

Each sample of $x_{S C}(n)$ is a weighted sum of $M$ data symbols with the weights decaying in magnitude approximately as $1 / k . x_{O F D M}(n)$ is the sum of $M$ equal-magnitude data symbols. This accounts for its higher PAPR than that of $x_{S C}(n)$.

\subsubsection{Flexibility of Spectrum Assignment}

Cognitive radio systems, that share spectrum efficiently among different users and different systems, should be able to transmit signals with spectra that avoid interference with other users' spectra [23]. From the flexibility of spectrum assignment point of view, OFDM system clearly has the advantage. The SC modulated data symbols occupy spectrally the entire data signal bandwidth, while each individual data symbol of an OFDM system modulates a single sub-carrier [20,22]. Consider block-wise transmission system with cyclic prefix (CP). The discrete Fourier transform (DFT) of a block (with size $M$ ) of SC modulated data symbols can be written as

$$
A_{\ell}^{S C}=\frac{1}{\sqrt{M}} \sum_{m=0}^{M-1} a_{m} e^{-j \frac{2 \pi m \ell}{M}}, \quad \ell=0,1, \ldots, M-1
$$

where $a_{m}$ is the $m$ th modulated data symbol. For OFDM, we have

$$
A_{\ell}^{O F D M}=a_{\ell}, \quad \ell=0,1, \ldots, M-1 .
$$


From (2.4), since each data symbol occupies exactly one frequency, data symbols in an OFDM system can be efficiently assigned to different frequencies with different powers. However, from (2.3), each frequency of the SC modulated signal is a function of all data symbols, which reduces the flexibility of spectrum assignment for SC signals.

\subsubsection{Bit Error Rate Performance}

It has been shown that the uncoded BER performance of a $\mathrm{SC}$ system with $\mathrm{CP}$ using frequency domain equalizer outperforms the OFDM with CP [24,25] because each data symbol is spread over all frequencies, and hence benefits from frequency diversity. For coded SC and OFDM systems, OFDM often uses a powerful coding scheme, such as bit-interleaved coded modulation $(\mathrm{BICM})^{1}[26]$, while SC systems may employs a coded modulation scheme, such as pragmatic trellis coded modulation (TCM) [27] with symbol interleaving. Given the channel state information (CSI) at the transmitter, adaptive bit loading combined with adaptive coding and power allocation to the subcarriers give OFDM unrivaled BER performance on frequency selective channels. Comparisons of coded linear SC systems using frequency domain equalization with non-adaptively loaded coded OFDM systems have shown that the two systems offer similar, but not identical, BER performance $[24,28,30]$, for the same averaged SNR [21, pp. 138]. The BER performance of coded SC and OFDM depends on the code rates. For code rates of about $1 / 2$ or less, non-adaptively loaded coded OFDM shows a 0.5 to $1 \mathrm{~dB}$ average SNR gain over coded linearly equalized $\mathrm{SC}$ systems with frequency domain equalizers. Higher code rates tend to favor SC systems with frequency domain equalizers [21, pp. 139].

\footnotetext{
${ }^{1} \mathrm{BICM}$ can be, and is, used with SC also, but it cannot exploit variable SNR on each bit.
} 


\subsubsection{Quantization Effects and Phase Noise Sensitivity}

Consider the quantization of the transmitting signal. Since the OFDM signal has a larger dynamic range (larger PAPR), more bits are required to represent the signal, which implies larger word length for the implementation of the digital block in the transmitter. This word length has a major impact both on implementation cost and performance. As the word length decreases, the power consumption and complexity of digital blocks (such as the digital-to-analog (DAC) and all digital filters) decreases, but at the expense of quantization noise, hence the BER performance. Also the power consumption of analog blocks will decrease significantly as the dynamic range of the signal is lower [31].

Another consideration is the sensitivity to the frequency offset and phase noise, induced by the mobility of the user terminal or generated by the imperfect local oscillators (LO). It has been shown in [32] that for a given BER degradation, the values of the frequency offset and the linewidth of the carrier generator that are allowed for OFDM are orders of magnitude smaller than for SC systems carrying the same bit rate. The frequency offset and the phase noise induces inter-carrier interference [32]. For large number of sub-carriers, denoted as $M$, the SNR degradation due to frequency offset and phase noise can be well approximated by [32],

$$
D_{F O}= \begin{cases}\frac{10}{\ln 10} \frac{1}{3}\left(\pi M \frac{\Delta F_{c}}{R}\right)^{2} \frac{E_{s}}{N o} & \text { for OFDM } \\ \frac{10}{\ln 10} \frac{1}{3}\left(\pi \frac{\Delta F_{c}}{R}\right)^{2} & \text { for SC }\end{cases}
$$

and

$$
D_{P N}= \begin{cases}\frac{10}{\ln 10} \frac{11}{60}\left(4 \pi M \frac{\beta}{R}\right)^{2} \frac{E_{s}}{N o} & \text { for OFDM } \\ \frac{10}{\ln 10} \frac{1}{60}\left(4 \pi \frac{\beta}{R}\right)^{2} \frac{E_{s}}{N o} & \text { for SC, }\end{cases}
$$

respectively, where $\Delta F_{c}$ is the carrier offset, $R$ is a fixed symbol rate and $\beta$ is the oscillator linewidth. We see that a key difference for the SNR degradation between OFDM and SC is that the degradation for OFDM depends on the number of subcarriers $M$, while SC is independent of the number of sub-carriers. For wide area scenarios in the proposed future wireless system [79] (where user mobility is a key 
requirement), $M$ is proposed to be much larger than 100 . This can be one of the potential advantages for utilizing SC modulation in the uplink.

\subsubsection{Advantages of SC Modulation in the Uplink}

Some potential advantages, especially in the wide area scenario, of using SC modulation in the uplink for future broadband wireless communication systems can be summarized as follows [23, 29]:

- lower cost of power amplifier and lower battery drain due to reduced power backoff;

- less sensitive to phase noise induced by user mobility and imperfect LO;

- inherent diversity for the receiver through frequency-selectivity of the channel;

- SC modulation is a well-proven technology in many existing wireless and wireline applications and its RF system linearity requirements are well known. Some of the disadvantages of $\mathrm{SC}$ are remedied in this thesis: e.g. we show how spectrum flexibility can be achieved by frequency domain generation of SC signals in the GMC context, and we also show how the BER performance of SC on frequency selective channels can be boosted by iterative and turbo equalization.

\subsection{System Descriptions}

Fig. 2.2 depicts the overall system considered in this thesis, where a thicker line means complex signal flow and ' $\mathrm{S} / \mathrm{P}$ ' stands for serial to parallel conversion. We consider uncoded and coded frame-based single input single output (SISO) communications ${ }^{2}$, where a frame consists of $N_{B}$ blocks (Fig. 2.3). The blocks in the dashed boxes of Fig. 2.2 are removed for the uncoded case. Convolutional encoding is performed over one frame, while the modulated symbols are transmitted block by block ${ }^{3}$. A

\footnotetext{
${ }^{2}$ Sometimes it is also called packet-based communications.

${ }^{3}$ In commonly used OFDM terminology, a block is referred to as an OFDM symbol. Thus an OFDM symbol conveys multiple $(M)$ data symbols.
} 
$\mathrm{CP}$ is added between blocks to avoid inter-block interference (IBI). A random block interleaver over a frame is used before the mapping of coded bits onto the signal constellation points. The SC signal is generated using the GMC transmission technique $[6,67,69]$. The detailed structure of the GMC transmitter and receiver with the multiplexing of FDM pilots will be given in the following section. We assume independent fading channel realizations every frame, and the channel impulse response (CIR) is static within one block (or an OFDM symbol). The time-varying nature of the channel is realized on a block-by-block basis. The description of the time-varying frequency selective fading channels will be given in Sec. 2.3.3. The soft Viterbi decoding algorithm is used in the decoder for data sink.

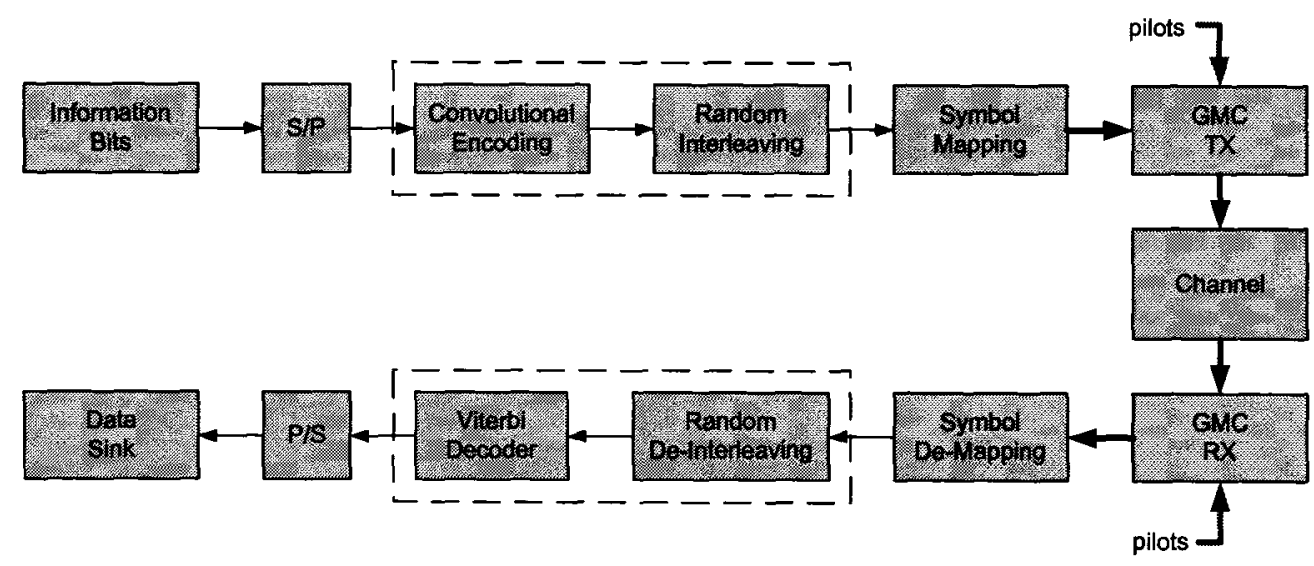

Figure 2.2: Overall System Block Diagram

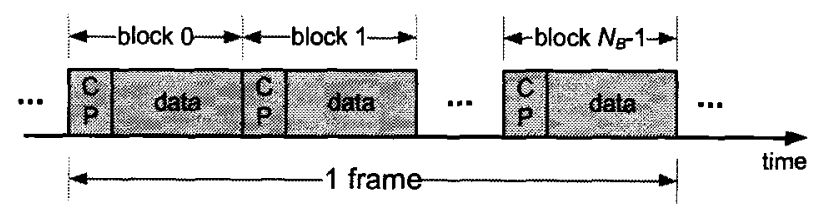

Figure 2.3: Definition of a Frame with Multiple Blocks 


\subsubsection{GMC Transmitter and Receiver with FDM Pilots}

Fig. 2.4 shows the block diagram of the transmitter and receiver using GMC signal generation [21]. We omit the block index for simplicity. The transmitted block, containing data and pilot symbols, consists of $L$ samples plus a $L_{c p}$ cyclic prefix, which is assumed to be longer than the channel impulse response (CIR). The complex data symbol $a_{m}$ has zero mean and unit variance. We assume a size- $M$ data plus pilot symbol block transmission $(M<L)$. The data tones $A_{\ell}$, (obtained by subjecting the original block of data symbols to a FFT operation) and pilot tones $P_{\ell}\left(N\right.$-point $\mathrm{FFT}^{4}$ of Chu sequence $\left\{c_{k}\right\}_{k=0}^{N-1}$ ) are multiplexed into a single frequency domain sequence, denoted as $X_{\ell}$ of length $M$. Note that $N<M<L$. The $k$ th element of a length- $N$ Chu sequence is given by [112]

$$
c_{k}= \begin{cases}e^{j \pi r k^{2} / N}, & \text { for } N \text { even } \\ e^{j \pi r k(k+1) / N}, & \text { for } N \text { odd }\end{cases}
$$

where $r$ is relatively prime to $N$. For equidistant pilot spacing, each group of data has the same size. The signal containing only all the equidistant pilot tones can be regarded as an interleaved frequency division multiple access (IFDMA) signal [78], which will be described in the next section. By padding enough zeros in the frequency domain to make a total length of $L$ and taking the IFFT, it is equivalent to use a sinc pulse for pulse shaping in the time domain with an oversampling factor of $I$ if $L=M I$. After adding the CP to prevent IBI, a time domain window can be added to reduce the side-lobes of the transmitted spectrum.

In the receiver side, before removing the $\mathrm{CP}$, a cyclic shift of the received samples is necessary due to the rolloff of the time window skirt. Taking the $L$ - point FFT of the received baseband sample $y_{n}$ and then removing the last $L-M$ frequencies, we obtain the received pilot and data tones

$$
Y_{\ell}=X_{\ell} H_{\ell}+V_{\ell}, \quad \ell=0,1, \ldots, M-1,
$$

\footnotetext{
${ }^{4}$ Note that FFT/IFFT is an efficient way of implementing DFT/IDFT [111].
} 


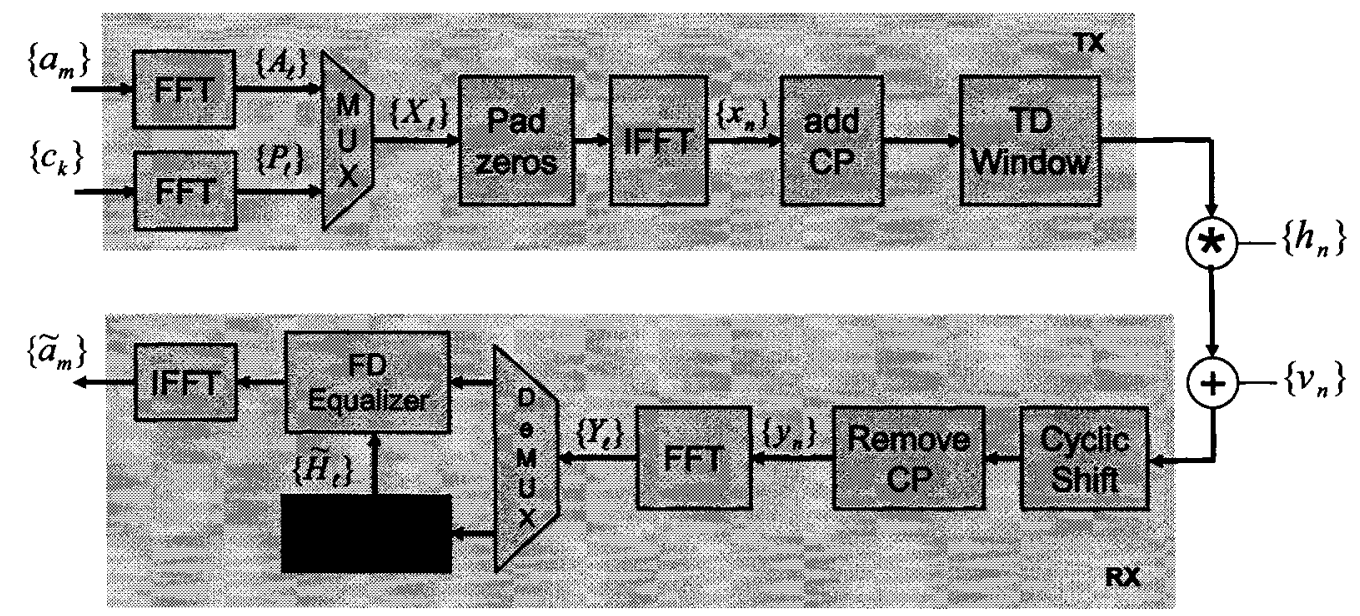

Figure 2.4: GMC Transmitter and Receiver Structure with FDM Pilots

where $H_{\ell}$ and $V_{\ell}$ represent the channel response at frequency $\ell$ and frequency domain noise sample at frequency $\ell$, respectively. The estimated data symbol $\tilde{a}_{m}$ is obtained by taking the IFFT of the frequency domain equalizer output. Frequency domain LMMSE equalization $[24,41]$ and iterative block soft decision feedback equalization (IBSDFE) [42] are considered throughout the thesis. A brief description of the frequency domain equalizers will be given in Sec. 2.4. Given the pilot symbols $P_{\ell}$, a frequency domain channel estimator (FDCE) is used to estimate the channel frequency response, denoted as $\tilde{H}_{\ell}$, for frequency domain equalizer. The 'FDCE' block (including the FDM pilot arrangement schemes in the transmitter) and the 'FD (frequency domain) Equalizer' block are the main focus of this thesis.

\subsubsection{IFDMA Training Signal}

IFDMA is a spread spectrum multiple access scheme based on frequency domain orthogonal signature sequences (FDOSS) $[75,77,78] .{ }^{5}$ While IFDMA was originally conceived as a multi-user spread spectrum signal format which preserves user orthogonality on frequency-selective channels, we use it here as a way of generating

\footnotetext{
${ }^{5}$ IFDMA has had many names, such as frequency domain orthogonal signature sequence (FDOSS) CDMA [77].
} 
frequency domain pilots with uniform frequency spacing. The IFDMA signal can be generated either in time domain [75] or in frequency domain [77]. We are interested in frequency domain generation of the IFDMA signal. Consider transmitting a block of $M_{c}$ chips with $N_{d}$ data symbols for the $k$ th user, denoted as $\left\{c_{n}^{(k)}\right\}_{n=0}^{N_{d}-1}$. The number of accommodated users ${ }^{6}$ is equal to the spreading factor $K_{s}$, given as $K_{s}=M_{c} / N_{d}$. The IFDMA signal can be generated by taking the $M_{c}$-point IDFT of the $N_{d}$ data frequencies equally interleaved over $M_{c}$ frequencies with $K_{s}$ different starting frequencies, given as

$$
\mathbf{x}_{P}^{(k)}=\mathbf{W}_{M_{c} \times M_{c}}^{H} \mathbf{X}_{P}^{(k)}, \quad k=0,1, \ldots, K_{s}-1
$$

where $\mathbf{W}_{M_{c} \times M_{c}}^{H}$ is the $M_{c} \times M_{c}$ IDFT matrix, the superscript ${ }^{H}$ denotes the Hermitian transpose and the $\ell$ th element of $\mathbf{X}_{P}^{(k)} \in \mathbb{C}^{M_{c} \times 1}$ is given as [77]

$$
X_{P}^{(k)}(\ell)= \begin{cases}C_{\ell^{\prime}}^{(k)}, & \ell=\ell^{\prime} K_{s}+k \\ 0, & \text { otherwise }\end{cases}
$$

where $C_{\ell}^{(k)}$ is the $N_{d}$-point DFT of $\left\{c_{n}^{(k)}\right\}_{n=0}^{N_{c}-1}$. Notice from $(2.10)$ that $\left\{X_{P}^{(k)}(\ell), \ell=\right.$ $\left.0,1, \ldots, M_{c}-1\right\}$ are equidistant line spectra with distance $K_{s}$ between two adjacent lines, as shown in the examples of IFDMA signals in Fig. 2.5. It can be shown that the IFDMA signals $\mathbf{x}_{P}^{(k)}$ for $k=0,1, \ldots, K_{s}-1$ are orthogonal signals and that the IFDMA signal has the same PAPR property as the SC signals [75]. Hence the IFDMA signal can be used as low PAPR training signal for SC system when equal-distance pilot tones are multiplexed in the frequency domain. Since optimum channel estimation using pilot symbols requires that the training signal has a flat spectrum and the low PAPR requires that the envelope of the pilot signal be constant, a Chu sequence [112] satisfies both conditions. Hence the IFDMA signal using a Chu sequence is chosen as the training signal, which can be generated using (2.9) with $\left\{c_{n}^{(k)}\right\}_{n=0}^{N-1}$ being the length- $N$ Chu sequence (see (2.7)). Note that we have $K_{s}$ different starting frequencies for the pilot tones. The IFDMA signal can also be considered as a special

\footnotetext{
${ }^{6}$ We consider here all users have the same spreading factor although users can have different spreading factors as long as $M_{c}=K_{s}^{\prime} N_{d}^{\prime}$, where $K_{s}^{\prime} \neq K_{s}$ and $N_{d}^{\prime} \neq N_{d}$ [77].
} 


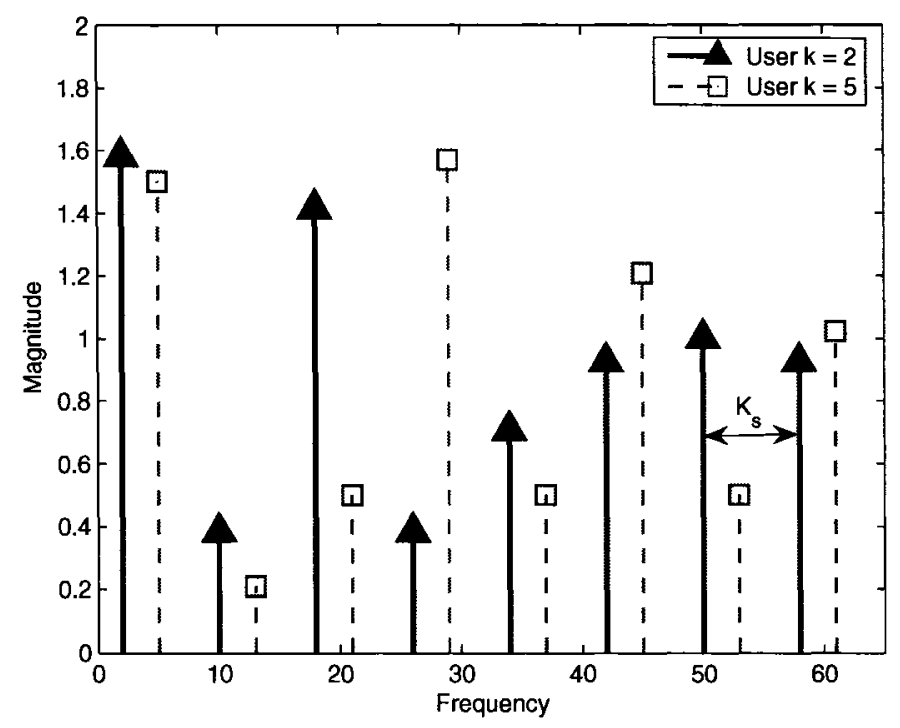

Figure 2.5: Magnitude Response of IFDMA Signals with $M_{c}=64, N_{c}=8$ and $K_{s}=8$

case of the chunk size system (where users occupy partial signal bandwidth), which will be discussed in Chapter 6. In Appendix B, we compare the performance between the IFDMA and DS-CDMA systems in the presence of carrier frequency offsets and Wiener phase noise.

\subsubsection{Time-varying Multipath Channel Model}

The complex baseband time-varying multipath channel can be described by $[35,36]$

$$
h(t, \tau)=\sum_{q=1}^{Q_{0}} c_{q}(t) \delta\left(\tau-\tau_{q}(t)\right)
$$

where $c_{q}(t)$ is the time-varying complex gain of the $q$ th path, $\tau_{q}(t)$ is the $q$ th delay of the path and $Q_{0}$ is the number of paths. Consider a non-line-of-sight (NLOS) communication link. The random fluctuations of $c_{q}(t)$, arising from a large number of reflections and scattering from rough granular surfaces, can be modeled as a zero mean wide sense stationary Gaussian random process in time using the Central Limit Theorem [91]. The envelope of $c_{q}(t)$, denoted as $\mathbf{r}(t)=\left|c_{q}(t)\right|$, then has a Rayleigh 
probability density function, given as $[35,36,91]$

$$
f_{\mathbf{r}}(r)=\frac{r}{\sigma_{r}^{2}} e^{-\frac{r^{2}}{2 \sigma_{r}^{2}}}
$$

where $\sigma_{r}^{2}$ is the variance of $r$. Assuming a wide sense stationary uncorrelated scattering (WSSUS) model, first introduced by Bello [37], the auto-correlation of $c_{q}(t)$ in time $t$ can be calculated as

$$
\begin{aligned}
R_{c c}(\Delta t) & \triangleq E\left\{c_{q}(t+\Delta t) c_{q}^{*}(t)\right\} \\
& =\sigma_{q}^{2} R_{t}(\Delta t)
\end{aligned}
$$

where ${ }^{*}$ denotes complex conjugate, $\sigma_{q}^{2}$ is the average power of the $q$ th path and $R_{t}(\Delta t)$ represents the normalized auto-correlation of $c_{q}(t)$ in time. The Fourier transform of $R_{t}(\Delta t)$ with respect to $t$ gives the Doppler spectrum due to the time variations, caused by the relative motion between the base station and user terminal. We assume that all paths have the same classical Jakes' power spectrum [38], given as

$$
S(f)= \begin{cases}\frac{1}{\pi f_{D \max } \sqrt{1-\left(\frac{f}{f_{D \max }}\right)}}, & |f| \leq f_{D \max } \\ 0, & \text { otherwise }\end{cases}
$$

where $f_{D \max }$ is the one-sided maximum Doppler spread. Given the speed of the user terminal and the carrier frequency, $f_{D \max }$ can be easily obtained as [34]

$$
f_{D \max }=\frac{v \cdot f_{c}}{c}
$$

where $v$ is the speed of the user terminal in $[\mathrm{m} / \mathrm{s}], f_{c}$ the carrier frequency in $[\mathrm{Hz}]$ and $c=3.0 \times 10^{8}[\mathrm{~m} / \mathrm{s}]$ is the speed of light. Taking the inverse Fourier Transform of (2.14), $R_{t}(\Delta t)$ can be found as

$$
R_{t}(\Delta t)=J_{0}\left(2 \pi f_{D \max } \Delta t\right)
$$

where $J_{0}(\cdot)$ is the zeroth-order Bessel function of the first kind. 
By taking the Fourier transform of (2.11) with respect to $\tau$, the multipath timevarying channel frequency response can be obtained as

$$
\begin{aligned}
H(t, f) & \triangleq \int_{-\infty}^{\infty} h(t, \tau) e^{-j 2 \pi f \tau} d \tau \\
& =\sum_{q=1}^{Q_{0}} c_{q}(t) e^{-j 2 \pi f \tau_{q}} .
\end{aligned}
$$

The auto-correlation of (2.17) in time and frequency can then be calculated as [39]

$$
\begin{aligned}
R_{H H}(\Delta t, \Delta f) & \triangleq E\left\{H(t+\Delta t, f+\Delta f) H^{*}(t, f)\right\} \\
& =\sum_{q=1}^{Q_{0}} R_{c c}(\Delta t) e^{-j 2 \pi \Delta f \tau_{q}} \\
& =R_{t}(\Delta t) R_{f}(\Delta f)
\end{aligned}
$$

where $R_{f}(\Delta f)$ is defined as

$$
R_{f}(\Delta f)=\sum_{q=1}^{Q_{0}} \sigma_{q}^{2} e^{-j 2 \pi \Delta f \tau_{q}} .
$$

Note that we normalize the total average power of the channel impulse response to unity, i.e. $\sum_{q=1}^{Q_{0}} \sigma_{q}^{2}=1$. From (2.18), we see that the auto-correlation of $H(t, f)$ can be expressed as a product of a time domain correlation $R_{t}(\Delta t)$ and a frequency domain correlation $R_{f}(\Delta f)$. This separability is the key to the channel estimation using cascaded 1-dimensional (1D) finite impulse response (FIR) Wiener filtering $[40,81]$, which is used throughout the thesis and will be described in Sec. 2.6.3.

In practice, when the channel statistics are not available or when real time calculation of auto- and cross-correlation of the CIR is prohibitive, we use a robust channel estimator [39], where we assume the Doppler spectrum and the power delay profile is uniform and only need to calculate the correlations of the channel once. The value of $R_{t}(\Delta t)$ for a uniform power spectrum can be found as [74]

$$
R_{t}^{U}(\Delta t)=\operatorname{sinc}\left(2 f_{D \max } \Delta t\right)
$$

where $\operatorname{sinc}(x)=\frac{\sin (\pi x)}{\pi x}$ and $f_{D \max }$ is the one-sided maximum Doppler frequency. The value of $R_{f}(\Delta f)$ for a uniform power delay profile with a one-sided maximum delay 
spread, denoted as $\tau_{\max }$, can be found as [74]

$$
R_{f}^{U}(\Delta f)=\operatorname{sinc}\left(2 \pi \tau_{\max } \Delta f\right)
$$

Note that in the discrete time domain, $\Delta t$ is the sampling period and $\Delta f$ is the subcarrier spacing.

\subsubsection{Simulation System Parameters}

In this section, we summarize different sets of system parameters and channel power delay profiles used throughout the thesis. Since channels are modeled according to different scenarios, we focus on the wide area (urban macro) scenario, where high mobility is a key requirement [61]. Table 2.1 shows the first urban-macro channel used in the WINNER Project [79] and the corresponding WINNER system parameters are shown in Table 2.2. The channel model has 18 paths and the maximum delay spread is $4.625 \mu \mathrm{s}$. The number of symbols per block is 826 symbols and the maximum Doppler frequency is $324 \mathrm{~Hz}$. We call parameters in Table 2.1 and Table 2.2 system parameters Set 1 for convenience. Table 2.3 and Table 2.4 show slightly different sets of system parameters adopted later by WINNER. The channel model now has 20 paths and the maximum delay spread is $1.47 \mu s$, about 3 times smaller than that for parameter Set 1 . We call parameters in Table 2.3 and Table 2.4 system parameters Set 2 for convenience. There are two subsets of parameter values for parameters Set 2. The main difference between the two subsets of parameters are the carrier frequency $(4.2 \mathrm{GHz}$ and $3.7 \mathrm{GHz}$ ) and the number of subcarriers in use (416 and 1024). Moreover, their signal bandwidths $(16.25 \mathrm{MHz}$ and $40 \mathrm{MHz})$ are different. We labeled them parameters Set 2-A and Set 2-B correspondingly. Note that Set 1 has higher maximum Doppler frequency and larger cyclic prefix length than that for Set 2. Both parameters Set 1 and Set 2 are targeting wide-area applications. 


\section{Definition of SNR}

The SNR used throughout this thesis is defined as the ratio of total average received power from data, pilots and cyclic prefix to total noise power in the signal bandwidth, given as

$$
\mathrm{SNR}=\frac{E_{s}}{N_{0}} \cdot \frac{\left(N_{\text {data }}+N_{p} \cdot S_{p}\right)\left(1+\frac{L_{c p}}{N_{F F T}}\right)}{\left(N_{\text {data }}+N_{p}+L_{c p}\right)}
$$

where $\frac{E_{s}}{N_{0}}$ is the ratio of energy per coded data symbol to the noise power in the signal bandwidth, $N_{\text {data }}$ is the number of data symbols per coded frame, $N_{p}$ is the number of pilots per coded frame, $S_{p}$ is the pilot boost factor, $L_{c p}$ is the length of the cyclic prefix, and $N_{F F T}$ is the FFT size, defined as $N_{F F T}=N_{d a t a}+N_{p}$.

\section{Definition of $\frac{E_{b}}{N_{0}}$}

In order to take into account the pilot overhead in the calculation of $\frac{E_{b}}{N_{0}}$, we use the following definition, given as

$$
\frac{E_{b}}{N_{0}}=\frac{E_{s}}{N_{0}} \cdot \frac{\left(N_{\text {data }}+N_{p} \cdot S_{p}\right)\left(1+\frac{L_{c p}}{N_{F F T}}\right)}{N_{\text {data }} \cdot r \log _{2}(\Lambda)}
$$

where $\frac{E_{b}}{N_{0}}$ is the ratio of energy per data bit to the noise power in the signal bandwidth, $r$ is the channel coding rate and $\Lambda$ is the constellation size. Note that we assume $S_{p}=1$ in this thesis. If $S_{p}=1$, it can be shown that $\mathrm{SNR}=\frac{E_{s}}{N_{0}}$ so that (2.23) can also be used to relate $\frac{E_{b}}{N_{0}}$ and SNR, given as

$$
\frac{E_{b}}{N_{0}}=\mathrm{SNR} \cdot \frac{\left(N_{d a t a}+N_{p}\right)\left(1+\frac{L_{c p}}{N_{F F T}}\right)}{N_{d a t a} \cdot r \log _{2}(\Lambda)} .
$$

\begin{tabular}{|c|c|}
\hline Power $[\mathrm{dB}]$ & 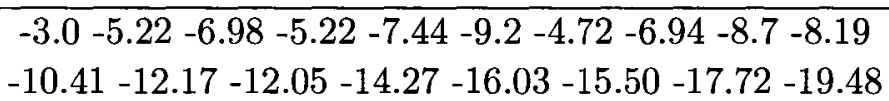 \\
\hline Delays $[\mu s]$ & 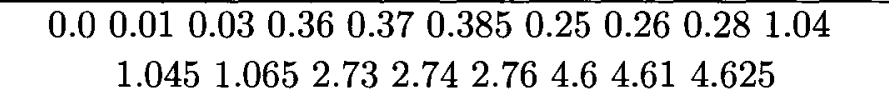 \\
\hline
\end{tabular}

Table 2.1: Urban Macro Power Delay Profile Set 1 [79] 
Table 2.2: Simulation System Parameters Set 1 [79]

\begin{tabular}{|c|c|}
\hline Parameter & Value \\
\hline Carrier frequency $[\mathrm{GHz}]$ & 5.0 \\
\hline Duplex Mode & FDD \\
\hline System bandwidth $[\mathrm{MHz}]$ & 20.0 \\
\hline Modulated symbols per block $(M)$ & 826 \\
\hline Symbol rate [Msps] & 16.25 \\
\hline Channel coding (convolutional) & $(133,171)_{O}$ \\
\hline Time domain windowing rolloff factor[\%] & 5.3 \\
\hline Modulation & QPSK \\
\hline Upsampling factor $(I)$ & 12 \\
\hline Number of blocks per frame $\left(N_{B}\right)$ & 10 \\
\hline Maximum Doppler Frequency $[\mathrm{Hz}]$ & 324 \\
\hline Cyclic prefix length $[\mu \mathrm{s}]$ & 5.00 \\
\hline
\end{tabular}

Table 2.3: Urban Macro Power Delay Profile Set 2 [67]

\begin{tabular}{|c|c|}
\hline Power $[\mathrm{dB}]$ & 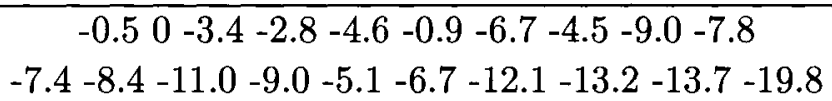 \\
\hline & 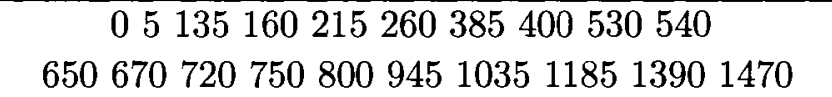 \\
\hline
\end{tabular}

Table 2.4: Simulation System Parameters Set 2

\begin{tabular}{|c|c|c|}
\hline Parameter & Value A [67] & Value B [68] \\
\hline Carrier frequency $[\mathrm{GHz}]$ & 4.2 & 3.7 \\
\hline Duplex Mode & FDD & FDD \\
\hline Subcarrier spacing $[\mathrm{Hz}]$ & 39062.5 & 39062.5 \\
\hline Cyclic prefix length $[\mu \mathrm{s}]$ & 3.2 & 3.2 \\
\hline Total symbol length $[\mu \mathrm{s}]$ & 28.80 & 28.80 \\
\hline Number of subcarriers in use $(M)$ & 416 & 1024 \\
\hline Signal BW [MHz] & 16.25 & 40.0 \\
\hline Time domain windowing rolloff factor[\%] & 8.3 & 5.5 \\
\hline Modulation & QPSK & QPSK \\
\hline Upsampling factor $(I)$ & 10 & 5 \\
\hline Maximum Doppler Frequency $[\mathrm{Hz}]$ & 194.4 & 171.3 \\
\hline Number of blocks per frame $\left(N_{B}\right)$ & 12 & 12 \\
\hline Channel coding (convolutional) & $(133,171)_{O}$ & $(133,171)_{O}$ \\
\hline
\end{tabular}




\subsection{Frequency Domain Channel Equalization for SC Systems}

Frequency domain channel equalization for SC systems is considered in $[24,28,29,41$, 42]. We consider a frequency domain linear minimum mean square error (LMMSE) equalizer and an iterative block soft decision feedback equalizer (IBSDFE) equalizer, similar to the equalizer proposed in [42]. Note that frequency domain block iterative equalizer using hard decisions is considered in [53].

\subsubsection{Linear MMSE Channel Equalization}

Fig. 2.6 shows the general structure of a frequency domain linear equalizer. The MSE

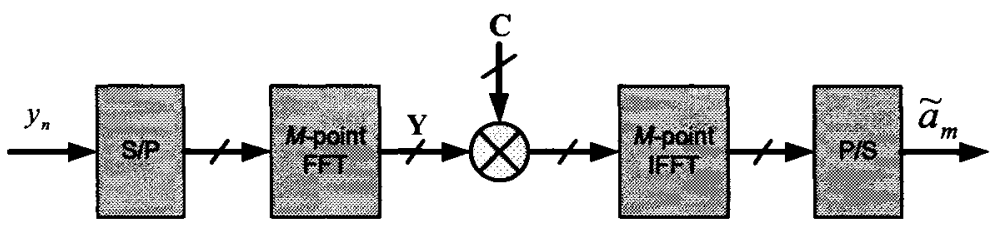

Figure 2.6: Structure of a Frequency Domain LMMSE Equalizer

of a frequency domain linear equalizer output can be calculated as

$$
\begin{aligned}
J & =E\left\{\left|a_{m}-\tilde{a}_{m}\right|^{2}\right\} \\
& =\frac{1}{M} \sum_{\ell=0}^{M-1} E\left\{\left|A_{\ell}-\tilde{A}_{\ell}\right|^{2}\right\} \\
& =\frac{1}{M} \sum_{\ell=0}^{M-1}\left|C_{\ell} H_{\ell}-1\right|^{2}+\frac{\sigma^{2}}{M} \sum_{\ell=0}^{M-1}\left|C_{\ell}\right|^{2}
\end{aligned}
$$

where $a_{m}$ is the data symbol, $\tilde{a}_{m}$ is the estimate of the $m$ th data symbol from the equalizer, $A_{\ell}$ is the $M$-point DFT of $a_{m}, \tilde{A}_{\ell}$ is the $M$-point DFT of $\tilde{a}_{m}, H_{\ell}$ is the actual channel frequency response, $\sigma^{2}$ is the white noise variance and $C_{\ell}$ is the $\ell$ th frequency domain equalizer tap of $\mathbf{C} \in \mathbb{C}^{M \times 1}$. We assume that $E\left\{\left|A_{\ell}\right|^{2}\right\}=E\left\{\left|a_{m}\right|^{2}\right\}=1$. For frequency domain LMMSE equalizer, the optimum equalizer taps, in terms of MSE, 
can be found as [24]

$$
C_{\ell}=\frac{H_{\ell}^{*}}{\left|H_{\ell}\right|^{2}+\sigma^{2}}, \quad \ell=0,1, \ldots, M-1
$$

where ${ }^{*}$ denotes the complex conjugate. Notice that the filter taps require the full bandwidth channel frequency response (CFR) $\left\{H_{\ell}\right\}_{\ell=0}^{M-1}$ and the noise variance $\sigma^{2}$. By substituting (2.26) into (2.25), the minimum MSE of the equalizer output when the channel frequency response is known at the receiver can be found as

$$
J_{o}=\frac{1}{M} \sum_{\ell=0}^{M-1} \frac{\sigma^{2}}{\left|H_{\ell}\right|^{2}+\sigma^{2}} .
$$

\subsubsection{Iterative Block Soft Decision Feedback Equalization}

To further improve the system performance, we consider low complexity IBSDFE, which alleviates the issue of error propagations usually occurring in a time domain decision feedback equalizer. Fig. 2.7 shows the structure of the frequency domain IBSDFE proposed in [42]. The feedforward filter taps are denoted as $\mathbf{C} \in \mathbb{C}^{M \times 1}$,

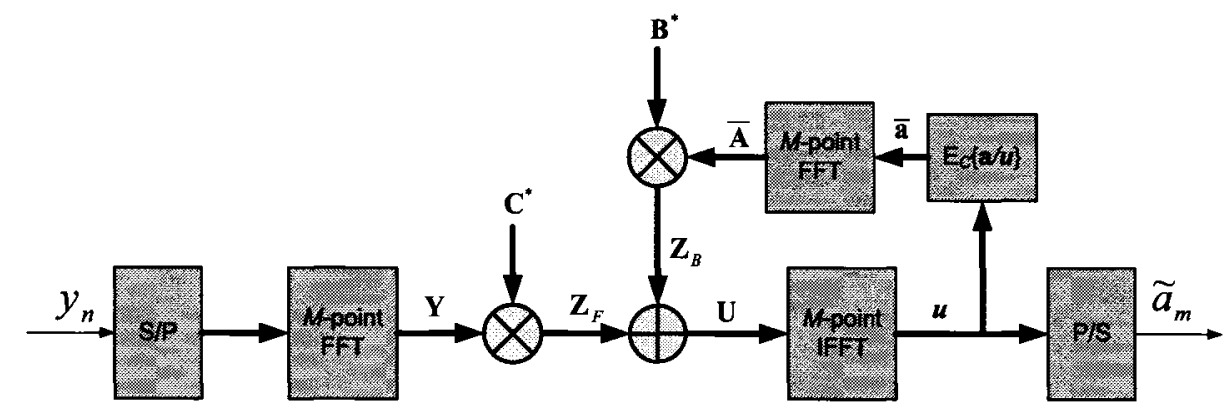

Figure 2.7: Structure of A Frequency Domain Iterative Block Soft Decision Feedback Equalizer

while the feedback filter taps are denoted as $\mathbf{B} \in \mathbb{C}^{M \times 1}$. After taking the DFT of the received signal $\left\{y_{n}\right\}_{n=0}^{M-1}$, the received frequency samples $\mathbf{Y} \in \mathbb{C}^{M \times 1}$ are elementby-element multiplied by $\mathbf{C}^{*}$ to obtain the equalized frequency samples $\mathbf{Z}_{F}$. In the 0th iteration, the estimated data symbols $\tilde{\mathbf{a}}$ are obtained by taking the IDFT of $\mathbf{Z}_{F}=\mathbf{U}=\mathbf{Y} \cdot \mathbf{C}$, assuming there is no contribution from the feedback loop, i.e. 
$\mathbf{Z}_{B}=\mathbf{0}$. This is just a linear MMSE equalizer, as described in the previous section. Hence, the $\ell$ th element of $\mathbf{C}$ is calculated using (2.26). Starting from the $i$ th iteration $(i \geq 1)$, the feedback loop is used. Hence,

$$
\begin{aligned}
\mathbf{U}^{(i)} & =\mathbf{Z}_{F}^{(i)}+\mathbf{Z}_{B}^{(i)} \\
& =\mathbf{Y} \cdot \mathbf{C}^{(i) *}+\mathbf{B}^{(i) *} \cdot \overline{\mathbf{A}}^{(i-1)}, \quad i=1,2, \ldots
\end{aligned}
$$

where ${ }^{*}$ is the complex conjugate and $\overline{\mathbf{A}}^{(i-1)}$ is the DFT of the estimated data symbols from the $(i-1)$ th iteration, using a conditional mean estimator, given as

$$
\bar{a}_{m}^{(i-1)}=E\left\{a_{m} / \mathbf{u}^{(i-1)}\right\}, \quad m=0,1, \ldots, M-1
$$

where $\mathbf{u}^{(i-1)}$ is the equalizer output at the $(i-1)$ th iteration. Assuming that the residual inter-symbol-interference (ISI) of the equalizer output is small, we assume the $m$ th output of the equalizer, denoted as $u_{m}$, is a Gaussian random variable with mean depending on the signal constellation points $\left\{\gamma_{\varsigma}\right\}_{\varsigma=1}^{\Lambda}$ and variance $\sigma_{i}^{2} . \Lambda$ is the constellation size. Hence the conditional mean of the data symbol $a_{m}$, given the equalizer ouput $\mathbf{u}^{(i-1)}$, can be calculated as [52]

$$
\begin{aligned}
\bar{a}_{m}^{(i-1)} & =E\left\{a_{m} / u_{m}^{(i-1)}\right\} \\
& =\frac{\sum_{\varsigma=1}^{\Lambda} \gamma_{\varsigma} p\left(u_{m}^{(i-1)} / \gamma_{\varsigma}\right) p\left(\gamma_{\varsigma}\right)}{\sum_{\varsigma=1}^{\Lambda} p\left(u_{m}^{(i-1)} / \gamma_{\varsigma}\right) p\left(\gamma_{\varsigma}\right)}
\end{aligned}
$$

where the conditional density function $p\left(u_{m}^{(i-1)} / \gamma_{\varsigma}\right)$ is given as,

$$
p\left(u_{m}^{(i-1)} / \gamma_{\varsigma}\right)=\frac{1}{\pi \sigma_{m}^{(i-1) 2}} e^{-\frac{\left|\gamma_{\varsigma}-u_{m}^{(i-1)}\right|^{2}}{\sigma_{m}^{(i-1) 2}}} .
$$

For QPSK, with $\gamma_{\varsigma}=\frac{1}{\sqrt{2}}( \pm 1 \pm j), E\left\{\mathbf{a} / \mathbf{u}^{(i-1)}\right\}$, denoted as $\overline{\mathbf{a}}^{(i-1)}$, can be found as

$$
\overline{\mathbf{a}}^{(i-1)}=\frac{1}{\sqrt{2}}\left(\tanh \left(\frac{\sqrt{2} \Re\left\{\mathbf{u}_{m}^{(i-1)}\right\}}{\sigma_{(i-1)}^{2}}\right)+j \tanh \left(\frac{\sqrt{2} \Im\left\{\mathbf{u}_{m}^{(i-1)}\right\}}{\sigma_{(i-1)}^{2}}\right)\right)
$$

where we assume that $\sigma_{m}^{(i-1) 2}=\sigma_{(i-1)}^{2}$, for $m=0,1, \ldots, M-1, \tanh (\cdot)$ is the hyperbolic tangent function, $\Re\{z\}$ is the real part of $z$ and $\Im\{z\}$ is the imaginary part of z. Assuming that for QPSK $\left|\gamma_{\varsigma}\right|=1$ for $\varsigma=1,2, \ldots, \Lambda$, it can be easily shown that

$$
E\left\{\left|a_{m}\right|^{2} / u_{m}^{(i-1)}\right\}=1, \quad m=0,1, \ldots, M-1
$$


The feedforward and feedback filter taps at the $i$ th iteration are found by minimizing the MSE between the actual and the estimated data symbols at the equalizer output, subject to the constraint that the gain of the channel plus forward filter for the $m$ th data symbol be equal to unity $^{7}$, given as

$$
\begin{aligned}
\left(\mathbf{B}_{o}^{(i)}, \mathbf{C}_{o}^{(i)}\right) & =\arg \min _{\mathbf{B}^{(i)}, \mathbf{C}^{(i)}}\left\{\frac{1}{M} \sum_{\ell=0}^{M-1} E_{c}\left\{\left|A_{\ell}-U_{\ell}^{(i)}\right|^{2}\right\}\right\} \\
& =\arg \min _{\mathbf{B}^{(i)}, \mathbf{C}^{(i)}}\left\{\frac{1}{M} \sum_{\ell=0}^{M-1} E_{c}\left\{\left|A_{\ell}-Y_{\ell} C_{\ell}^{(i) *}-B_{\ell}^{(i) *} \bar{A}_{\ell}^{(i-1)}\right|^{2}\right\}\right\}
\end{aligned}
$$

subject to

$$
\frac{1}{M} \sum_{\ell=0}^{M-1} C_{\ell}^{*} H_{\ell}=1
$$

Expanding the MSE term in (2.34) and using the Lagrange multiplier factor $\mu$, the optimum solution of feedback and feedforward filter taps can be found, by setting the derivatives equal to zero, to be

$$
B_{\ell}^{(i)}=1-H_{\ell}^{*} C_{\ell}^{(i)}, \quad \ell=0,1, \ldots, M-1
$$

and

$$
C_{\ell}^{(i)}=\frac{H_{\ell}\left(S_{\ell}^{(i)}-\mu^{(i)}\right)}{\left|H_{\ell}\right|^{2} S_{\ell}^{(i)}+\sigma^{2}}, \quad \ell=0,1, \ldots, M-1
$$

where for QPSK,

$$
S_{\ell}^{(i)}=1-\frac{1}{M} \sum_{m=0}^{M-1}\left|\bar{a}_{m}^{(i-1)}\right|^{2}
$$

and the Lagrange multiplier factor $\mu^{(i)}$ can be found as

$$
\mu^{(i)}=-\frac{\sum_{\ell=0}^{M-1} \frac{\sigma^{2}}{\left|H_{\ell}\right|^{2} S_{\ell}^{(i)}+\sigma^{2}}}{\sum_{\ell=0}^{M-1} \frac{\left|H_{\ell}\right|^{2}}{\left|H_{\ell}\right|^{2} S_{\ell}^{(i)}+\sigma^{2}}} .
$$

Using (2.34), (2.36) and (2.37), the minimum conditional MSE for QPSK at the $i$ th iteration can be then be found to be

$$
\sigma_{i}^{2}=-\mu^{(i)}
$$

\footnotetext{
${ }^{7}$ Note that in [42], this condition is not invoked.
} 


\subsection{Overview of Channel Estimation for SC Sys- tems}

Channel estimation using training (pilot) symbols, sometimes called data-aided channel estimation [49], is employed in current 3rd generation (3G) [5] and will likely be employed in the proposed future beyond $3 \mathrm{G}$ (B3G) wireless communication systems $[67,79]$. Although suboptimal and bandwidth consuming, channel estimation using training symbols remains attractive in practice because of the decoupling of symbol detection from channel estimation, which reduces complexity and relaxes the required identifiability conditions $[43,45]$. As mentioned in Chapter 1 , we focus on the channel estimation using pilot symbols for block-based SC systems in time-varying frequency selective fading channels. The pilot symbols for $\mathrm{SC}$ systems are traditionally time domain multiplexed (TDM) within or in between the transmission blocks. When the TDM pilot symbols are inserted periodically (in the time domain) to track the time-varying channels, it is also known as pilot symbol assisted modulation (PSAM) [45-48,50]. An analysis of BER performance for PSAM system in flat fading channel is given in [47] and frequency selective fading in [48], which consider continuous transmission. PSAM for block transmission for SC system in time-varying frequency selective fading channels is considered in $[28,45,50,51]$ and the references therein. Using the pilot symbols, classical least square (LS) or linear MMSE estimators [52] are then used to estimate the CIR. Since the estimation is performed in the time domain, it usually involves the pseudo-inversion of a matrix with the size of the pilot (known decision) symbols $[45,50]$. Brief descriptions of the time LS and LMMSE channel estimation for block transmission will be provided in Sec. 2.5.2.

Iterative decision-directed channel estimation (IDDCE) can be used to improve the performance of the channel estimation after the initial channel estimation using PSAM $[16,58]$. Soft decisions for the coded symbols from the channel decoder are

used as pilots to further improve the performance channel estimation, thus improving the overall system performance. In [16], a time domain decision-directed channel 
estimator is incorporated into the iterative process of a frequency domain equalizer to improve the channel estimates. The channel estimation involves the inverse of a matrix with size of the decisions. In [58], the authors propose an iterative filtering technique using the decisions, again, from the channel decoder to improve the channel estimates in time-varying flat fading channels. This iterative filtering technique is adapted in [56] for OFDM in time-varying frequency selective fading channels (see Sec. 2.6.4). Note that we include the description of channel estimation using TDM pilots in order to compare the performance with that of the proposed channel estimation techniques using FDM pilots.

\subsubsection{TDM Pilot Arrangements}

Fig. 2.8 shows the TDM pilot arrangement for a frame with $N_{B}$ blocks and CP. All pilot symbols are placed at the beginning of the block (packet) $[28,50,51]$ and not all the blocks within a frame are equipped with pilots to reduce the pilot overhead. For frequency domain processing of block transmission, a $\mathrm{CP}$ is inserted to avoid IBI and be able to use circular convolution [28]. Since we consider the low complexity DFT channel estimator [70,92], the periodic behavior of the training symbols is also required, resulting in extra training symbols as the $\mathrm{CP}$ of the training block, as shown in Fig. 2.8. If we fixed the block size to $M$ symbols, $\left(M-L_{c}-N\right)$ data symbols are transmitted for blocks with pilots, e.g. block\# 2 in Fig 2.8. The number of pilots within a block, denoted as $N$, depends on the maximum delay spread, while the number of blocks with pilots, denoted as $N_{b}$, depends on the maximum Doppler frequency. We will describe how to calculate the minimum values of $N$ and $N_{b}$ in Sec. 2.6.1 when we consider the FDM pilot arrangements for time-varying frequency selective fading channels. For blocks without pilots, interpolation filters, such as the DFT and Wiener filters, are used to estimate the channel frequency response. 


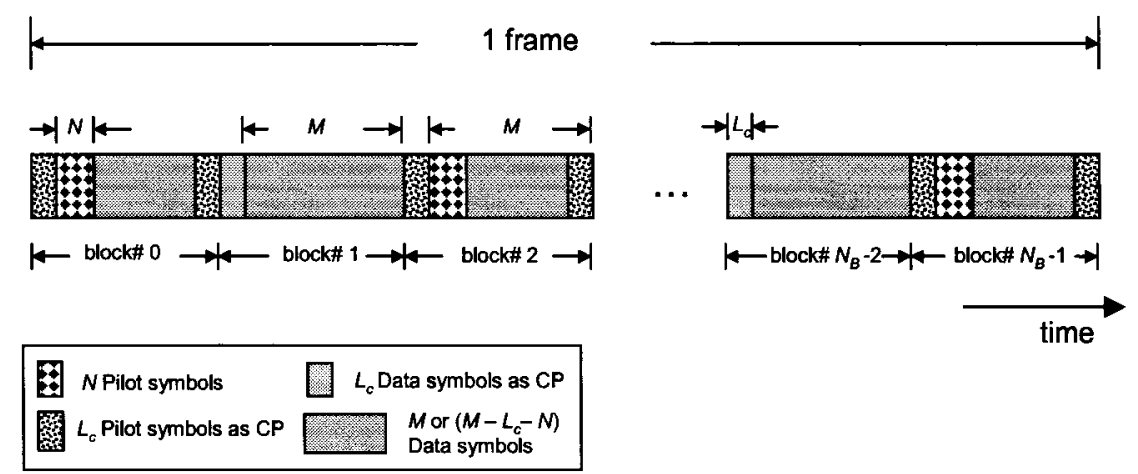

Figure 2.8: TDM Pilot Arrangements with CP

\subsubsection{Time Domain Least Square (LS) and LMMSE Channel Estimator}

The discrete time baseband received pilot (known decision) signal in the time domain can be expressed as

$$
\mathbf{y}=\Gamma \mathbf{h}+\mathbf{v}
$$

where $\boldsymbol{\Gamma} \in \mathbb{C}^{N \times N_{c h}}$ is a convolution matrix containing pilot (known decision) symbols, $\mathbf{h} \in \mathbb{C}^{N_{c h} \times 1}$ is the CIR and $\mathbf{v} \in \mathbb{C}^{N \times 1}$ is the noise vector. $N_{c h}$ is the number of discrete time channel samples. We assume that $N \geq N_{c h}$. The LS estimate of the CIR is given as [52]

$$
\tilde{\mathbf{h}}_{\mathbf{L S}}=\left(\boldsymbol{\Gamma}^{\mathbf{H}} \boldsymbol{\Gamma}\right)^{-1} \Gamma^{H} \mathbf{y}
$$

where ${ }^{H}$ is the Hermitian transpose and $\left(\boldsymbol{\Gamma}^{\mathbf{H}} \boldsymbol{\Gamma}\right)^{-1} \Gamma^{H}$ is also known as the pseudoinverse of $\boldsymbol{\Gamma}$. From (2.42), the LS channel estimator does not require statistics of the CIR and noise variance. By exploiting the statistics of the CIR and noise samples, LMMSE estimates of the CIR is given as [52]

$$
\tilde{\mathbf{h}}_{\text {LMMSE }}=\mathbf{R}_{\mathbf{h h}} \boldsymbol{\Gamma}^{\mathbf{H}}\left(\boldsymbol{\Gamma} \mathbf{R}_{\mathbf{h h}} \boldsymbol{\Gamma}^{H}+\sigma^{2} \mathbf{I}\right)^{-1} \boldsymbol{\Gamma}^{H} \mathbf{y}
$$

where $\mathbf{R}_{\mathbf{h h}}=E\left\{\mathbf{h h}^{H}\right\}$ is the auto-correlation matrix of the CIR and $\sigma^{2}$ is the noise variance. We see that from (2.42) and (2.43), the estimation of the CIR using a LS or LMMSE estimator requires the inversion of $N \times N$ matrix, which requires 
$\mathcal{O}\left(N^{3}\right)$ complex multiplications and additions. Note that the inverse matrix can be pre-computed since the pilots are known. However, in the case of decision-directed channel estimation, the inverse matrix needs to be updated on-line.

\subsection{Overview of Channel Estimation for OFDM Systems}

Channel estimation techniques for OFDM systems can be divided into two main categories, namely pilot-aided channel estimation $(\mathrm{PACE})^{8}$ and decision directed channel estimation. The PACE technique uses sparse pilots to obtain the LS estimates ${ }^{9}$ of the CFR at the pilot locations. We consider a rectangular time-frequency pilot grid for time-varying frequency selective channels, which will be described in the next section. Given the LS estimates, the overall channel frequency response is then obtained by interpolation filters, such as the DFT channel estimator [54,92] and cascaded 1D FIR Wiener filters $[72-74,81]$, as shown in Fig. 2.9. Given the received frequency samples

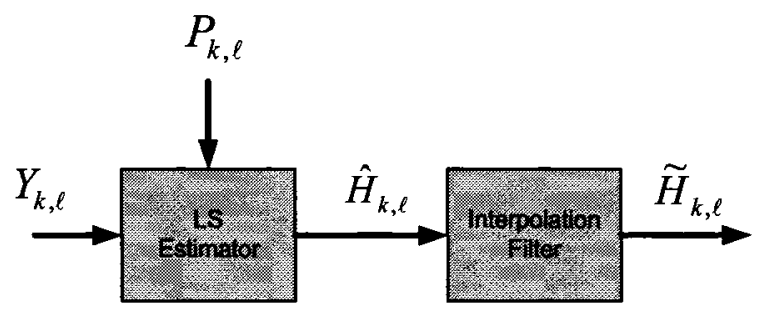

Figure 2.9: Typical Channel Estimation Techniques for OFDM Systems

at the $k$ th block $\mathbf{Y}^{(k)} \in \mathbb{C}^{M \times 1}$ with pilot frequency samples $\mathbf{P}^{(k)} \in \mathbb{C}^{M \times 1}$, the LS estimates can be calculated as

$$
\begin{aligned}
\hat{H}_{k, \ell} & =\frac{Y_{k, \ell}}{P_{k, \ell}} \\
& =H_{k, \ell}+V_{k, \ell}^{\prime}, \quad k \in \Psi \text { and } \ell \in \Omega
\end{aligned}
$$

\footnotetext{
${ }^{8}$ Sometimes it is also known as frequency domain PSAM [40].

${ }^{9}$ The LS estimates in this thesis are equivalent to the initial raw channel estimates at the pilot locations since the number of channel frequency response (CFR) samples to be estimated is the same as that of the noisy received frequency samples. They are also called a posteriori LS estimates in [40].
} 
where $V_{k, \ell}^{\prime}=\frac{V_{k, \ell}}{P_{k, \ell}}$ are statistically independent and identically distributed complex Gaussian noise samples at the pilot location scaled by the deterministic $(k, \ell)$ th pilot tone, $\Psi$ denotes the index of the blocks with pilots within a frame of size $N_{B}$, given as

$$
\boldsymbol{\Psi}=\left\{0, K_{t}, \ldots,\left(N_{b}-1\right) K_{t}\right\}
$$

where $K_{t}$ is the pilot distance (in blocks) in time and $\boldsymbol{\Omega}$ denotes the frequency locations of the pilots within the $k$ th block, given as

$$
\boldsymbol{\Omega}=\{0, K, \ldots,(N-1) K\}
$$

where $K$ is the pilot spacing and $N$ is the number of pilots per OFDM symbol. Note that we assume the starting position of the pilots is zero for (2.45) and (2.46). We consider the DFT and mis-matched Wiener channel estimators in this thesis for reasonable complexity and performance. Note however that other interpolation techniques are also available, such as polynomial interpolation techniques [62-64]. The low complexity DFT-based channel estimator acts like a sinc interpolation filter, requiring no channel statistics. In the other hand, the Wiener interpolation filter requires channel auto- and cross-correlations to minimize the expected MSE between the estimated and the actual channel frequency response. More detailed descriptions of both estimators will be provided in Sec. 2.6.2 and Sec. 2.6.3, respectively.

The decision-directed channel estimation technique uses the re-modulated data symbols (using the tentative decisions) to obtain the LS estimates [40]. Although better spectral efficiency can be achieved and more pilots are available for better interpolation performance, the performance of the decision-directed channel estimation heavily depends on the reliability of the data decisions, which depends on the accuracy of the channel estimates and the operating SNR range. To improve the reliability of the decisions, iterative channel estimation (ICE) using the log-likelihood ratio (LLR) of the coded symbols from the a posteriori (APP) decoder can be used [55-57]. The channel estimates in the 0th iteration is usually obtained by the PACE 
technique. With the aid of channel coding, more reliable decisions can be obtained for channel estimation for a lower range of SNRs. In terms of implementation complexity, it is still prohibitive to consider the optimum joint MAP data detection and channel estimation as the trellis expansion induced by the channel estimation task in the decoder enhances greatly the complexity of the conventional forward/backward procedure $[23,57]$. In this thesis, we consider suboptimum iterative decision directed channel estimation using 2-dimensional (2D) filtering techniques, separating the data detection and channel estimation processes. A low complexity iterative decision directed channel estimation technique for OFDM systems is described in Sec. 2.6.4. This channel estimator for OFDM systems is later modified for SC systems using FDM pilots, which will be described in Sec. 5.3.

\subsubsection{FDM Pilot Arrangements}

Fig. 2.10 shows an example of a $2 \mathrm{D}$ rectangular pilot grid in the time-frequency plane, where $N_{B}$ is the number of blocks per frame, $K$ is the subcarrier difference between two pilots, and pilots are added in the first and last block. Note that the

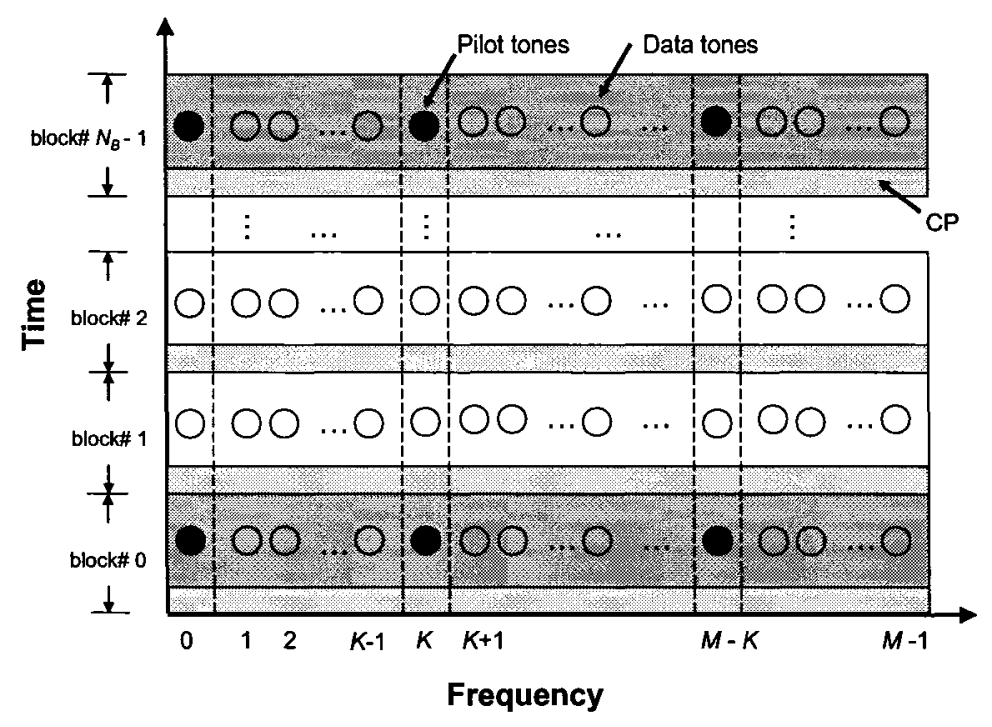

Figure 2.10: Time-Frequency Rectangular Pilot Grid 
optimum structure of the 2D pilot grid for a $2 \mathrm{D}$ Wiener interpolation filter was found to be a diamond shaped grid [65]. The rectangular grid should sample the frequency selectivity and time variation of the channel sufficiently often to allow adequate tracking performance. Let $D_{f}$ and $D_{t}$ denote the pilot distance in frequency direction and in time direction, respectively. Hence, in our example, we have $D_{f}=K$ and $D_{t}=N_{B}-1$. According to $2 \mathrm{D}$ sampling theorem, the values of $D_{f}$ and $D_{t}$ are fundamentally limited by the following conditions, given as $[66,74]$

$$
D_{f} \leq \frac{1}{\tau_{\max } \Delta F} \quad \text { and } \quad D_{t} \leq \frac{1}{2 f_{D \max } T_{B}}
$$

where $\tau_{\max }$ is the maximum delay spread, $\Delta F$ the subcarrier spacing, $f_{D \max }$ the maximum Doppler frequency and $T_{B}$ the time duration of the block including CP. Note that a factor of two in the denominator of $D_{f}$ is not included due to the assumption of single-sided power delay profile. In practice, an oversampling factor of two is usually used to achieve reasonable complexity and performance. Hence, the number of pilots $N$ and blocks with pilots $N_{b}$ can be calculated as

$$
N=\left\lceil\frac{M}{D_{f}}\right\rceil \text { and } \quad N_{b}=\left\lceil\frac{N_{B}}{D_{t}}\right\rceil
$$

where $\lceil\cdot\rceil$ is the ceiling function (rounded up to the next integer).

\subsubsection{DFT-Based Channel Estimator}

Fig. 2.11 depicts the general structure of a DFT-based channel estimator. Consider

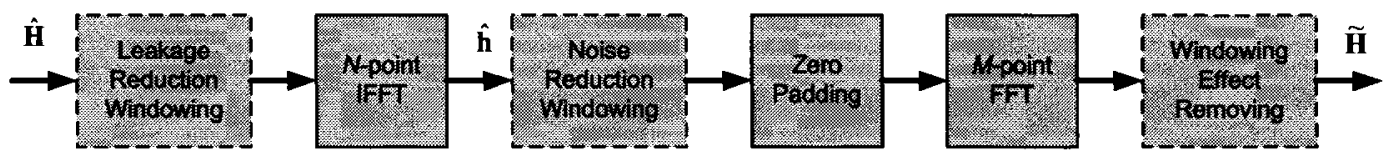

Figure 2.11: A General Structure of DFT-Based Channel Estimator

the solid-line blocks, bypassing the blocks of windowing. The estimated CIR, denoted as $\hat{\mathbf{h}}$, is found by taking the $N$-point IDFT of the LS estimated channel frequency 
response using pilot symbols (see (2.44)), given as

$$
\hat{\mathbf{h}}=\mathbf{W}_{N \times N}^{H} \hat{\mathbf{H}}
$$

where $\mathbf{W}_{N \times N}^{H}$ is the IDFT matrix and ${ }^{H}$ is the Hermitian transpose. Interpolation is used to obtain the channel frequency response between the pilot symbols, by simply padding zeros and taking the $M$-point DFT of the padded sequence, denoted as $\hat{\mathbf{h}}^{\prime}$. Hence,

$$
\tilde{\mathbf{H}}=\mathbf{W}_{M \times M} \hat{\mathbf{h}}^{\prime}
$$

where

$$
\hat{\mathbf{h}}^{\prime}= \begin{cases}{\left[\hat{h}_{0}, \hat{h}_{1}, \ldots, \hat{h}_{N-1}, \mathbf{0}_{1 \times(M-N)}\right]^{T},} & \tau_{q} / T_{s}=k \\ {\left[\hat{h}_{0}, \ldots, \hat{h}_{N-\Delta-1}, \mathbf{0}_{1 \times(M-N)}, \hat{h}_{N-\Delta}, \ldots, \hat{h}_{N-1}\right]^{T},} & \tau_{q} / T_{s} \neq k\end{cases}
$$

where $T_{s}$ is the sample time ${ }^{10}, k$ is an integer, $\tau_{q}$ is the $q$ th path delay, $q=1,2, \ldots, Q_{0}$ and $\Delta$ can be approximated by $[92]$

$$
\Delta=\frac{N-\tau_{\max } / T_{s}}{2}
$$

The modified zero padding (lower part of (2.51)) is used for non-sample spaced CIR, which results in the power of each tap being not localized at a certain position but being spread over the whole time range, an effect called leakage [92]. To further enhance the performance of the DFT-based channel estimation, windowing techniques, e.g. those considered in [54,92], can be used to reduce the leakage effects and smooth the estimated CIR before taking the $M$-point DFT. Note that in this thesis, the windowing techniques are not used.

\subsubsection{Cascaded 1D-FIR Wiener Channel Estimator}

Conceptually, the discrete shift-variant 2D Wiener filter is the optimal linear pilotaided estimator in terms of MSE for 2D PACE, however, two cascaded orthogonal 1D filters are simpler to implement and virtually as good as true 2D filters [81]. We

\footnotetext{
${ }^{10}$ In the SC context, this is called symbol time.
} 
start filtering in the frequency direction for a particular block with pilots, e.g. the $k_{0}$ th block. The channel estimates in the frequency direction can be obtained as,

$$
\tilde{H}^{\prime}\left(k_{0}, \ell\right)=\sum_{i=0}^{I_{f}-1} W_{f}^{(i)}\left(k_{0}, \ell\right) \hat{H}\left(k_{0}, \ell_{i}\right), \quad \ell_{i} \in \boldsymbol{\Omega} \text { and } k_{0} \in \Psi
$$

where $\hat{H}\left(k_{0}, \ell_{i}\right)$ is the LS estimates of the channel frequency response at the pilot locations, as defined in (2.44), $I_{f}$ is the number of the frequency direction filter taps. Note that $\Omega$ and $\Psi$ are defined in (2.46) and (2.45), respectively. $\left\{k_{0}, \ell_{i}\right\}$ are chosen such that they are the nearest in distance to the $\left(k_{0}, \ell\right)$ th frequency. The optimum solution of (2.53) in terms of MSE is the solution of the Wiener-Hopf equations [71], given as

$$
\begin{aligned}
\mathbf{W}_{f}\left(k_{0}, \ell\right) & =\left[W_{f}^{(0)}\left(k_{0}, \ell\right), W_{f}^{(1)}\left(k_{0}, \ell\right), \ldots, W_{f}^{\left(I_{f}-1\right)}\left(k_{0}, \ell\right)\right] \\
& =\mathbf{r}_{\Delta \ell}^{T}\left(k_{0}, \ell\right) \Phi_{f}^{-1}
\end{aligned}
$$

where the $i$ th element of $\mathbf{r}_{\Delta \ell}\left(k_{0}, \ell\right)$ is

$$
\begin{aligned}
r_{\Delta \ell}^{(i)}\left(k_{0}, \ell\right) & =E\left\{H\left(k_{0}, \ell\right) H\left(k_{0}, \ell_{i}\right)^{*}\right\} \\
& =r_{\Delta \ell}\left(\ell-\ell_{i}\right)
\end{aligned}
$$

where $\ell=0,1, \ldots, M-1$ and ${ }^{*}$ denotes complex conjugate. The $(i, j)$ th element of $\Phi_{f}$ is

$$
\begin{aligned}
\Phi_{f}(i, j) & =E\left\{H\left(k_{j}, \ell_{i}\right) H\left(k_{j}, \ell_{j}\right)^{*}\right\}+\sigma^{2} \delta\left(\ell_{i}-\ell_{j}\right) \\
& =r_{\Delta \ell}\left(\ell_{i}-\ell_{j}\right)+\sigma^{2} \delta\left(\ell_{i}-\ell_{j}\right)
\end{aligned}
$$

where $\ell_{i}, \ell_{j} \in \Omega$. Note that the selection of pilots for a particular $\ell$ is based on the absolute distance between the pilot frequency and frequency $\ell$. The MMSE of $\tilde{H}\left(k_{0}, \ell\right)$ can be calculated as $[52,71]$,

$$
\begin{aligned}
J_{o}\left(k_{0}, \ell\right) & =E\left\{\left|H_{k_{0}, \ell}-\tilde{H}_{k_{0}, \ell}^{\prime}\right|^{2}\right\} \\
& =1-\mathbf{r}_{\Delta \ell}^{T}\left(k_{0}, \ell\right) \Phi_{f}^{-1} \mathbf{r}_{\Delta \ell}^{*}\left(k_{0}, \ell\right) .
\end{aligned}
$$


Similar to the filtering in frequency direction, to filter in time direction, we fix the frequency index $\ell$. The channel estimates in the time direction can be found as

$$
\tilde{H}(k, \ell)=\sum_{j=0}^{I_{t}-1} W_{t}^{(j)}(k, \ell) \tilde{H}^{\prime}\left(k_{j}, \ell\right)
$$

where $I_{t}$ is the number of time direction filter taps and $k_{j} \in \Psi$. Similar to (2.54), the optimum solution can be calculated as,

$$
\begin{aligned}
\mathbf{W}_{t}(k, \ell) & =\left[W_{t}^{(0)}(k, \ell), W_{t}^{(1)}(k, \ell), \ldots, W_{t}^{\left(I_{t}-1\right)}(k, \ell)\right]^{T} \\
& =\mathbf{r}_{\Delta k}^{T}(k, \ell) \Phi_{t}^{-1}
\end{aligned}
$$

The $j$ th element of $\mathbf{r}_{\Delta \ell}(k, \ell)$ is

$$
\begin{aligned}
r_{\Delta k}^{(j)}(k, \ell) & =E\left\{H(k, \ell) H\left(k_{j}, \ell\right)^{*}\right\} \\
& =r_{\Delta k}\left(k-k_{j}\right)
\end{aligned}
$$

and the $(i, j)$ th element of $\boldsymbol{\Phi}_{t}$ is

$$
\begin{aligned}
\Phi_{t}(i, j) & =E\left\{H\left(k_{i}, \ell\right) H\left(k_{j}, \ell\right)^{*}\right\}+\frac{\sigma^{2}}{\alpha_{w}} \delta\left(k_{i}-k_{j}\right) \\
& =r_{\Delta k}\left(k_{i}-k_{j}\right)+\frac{\sigma^{2}}{\alpha_{w}} \delta\left(k_{i}-k_{j}\right)
\end{aligned}
$$

where $k_{i}, k_{j} \in \Psi$ and $\alpha_{w}$ is the SNR improvement factor due to the filtering in frequency direction, given as by trials [72]

$$
\alpha_{W} \approx \sqrt{\frac{I_{f}+I_{t}}{2}} .
$$

Similar to (2.57) the corresponding MMSE can be calculated as

$$
\begin{aligned}
J_{o}(k, \ell) & =E\left\{\left|H_{k, \ell}-\tilde{H}_{k, \ell}\right|^{2}\right\} \\
& =1-\mathbf{r}_{\Delta k}^{T}(k, \ell) \Phi_{t}^{-1} \mathbf{r}_{\Delta k}^{*}(k, \ell)
\end{aligned}
$$

Note that we only need to calculate (2.54) and (2.59) once if we assume the channel and noise statistics remain unchanged. This is possible if we assume a robust channel 
estimator which has a uniform (rectangular) power delay profile and a uniform (rectangular) Doppler power spectrum [39], for which the statistics may be mismatched to a wide range of the power delay profiles and Doppler spectrum. From (2.55), (2.56), (2.60) and (2.61), it is obvious that we need the cross- and auto-covariance of the actual channel. Using (2.20) and (2.21), the corresponding cross-covariances for a robust channel estimator with maximum delay spread $\tau_{\max }$ and maximum Doppler frequency $f_{D \max }$ can then be obtained as,

$$
\begin{aligned}
& r_{\Delta k}\left(k-k_{j}\right)=\operatorname{sinc}\left(2 f_{D_{\max }} T_{B}\left(k-k_{j}\right)\right) \\
& r_{\Delta \ell}\left(\ell-\ell_{i}\right)=\operatorname{sinc}\left(2 \tau_{\max } \Delta F_{s}(\ell-\ell i)\right)
\end{aligned}
$$

where $k=0,1, \ldots, N_{B}-1, \ell=0,1, \ldots, M-1, k_{j} \in \Psi, \ell_{i} \in \Omega, f_{D_{m} a x}$ is the one-sided maximum Doppler frequency, $\tau_{\max }$ is the one-sided maximum delay spread and $T_{B}$ is the data block plus the CP duration, $\Delta F$ is the carrier spacing. Using (2.64) and (2.65), the auto-covariance, $\Phi_{f}(i, j)$ and $\Phi_{t}(i, j)$ can be calculated according to $(2.56)$ and (2.61), respectively.

\subsubsection{Iterative Decision-Directed Channel Estimation}

Fig. 2.12 depicts a low complexity iterative channel estimator for OFDM systems based on filtering and decoding $[56]^{11}$. Initial channel estimates using a cascaded 1D-FIR Wiener filtering with pilot symbols are used by the soft I/O detector to calculate the input a posteriori likelihood ratio, denoted as $L_{\text {appost }}^{I n}$, given the input a priori likelihood ratio, denoted $L_{a p}^{I n}$ from the interleaver in the previous iteration. The input extrinsic information (denoted as $L_{\text {ext }}^{I n}$ ) is calculated as $L_{a p p o s t}^{I n}-L_{a p}^{I n}$. After the de-interleaving, $L_{e x t}^{I n}$ becomes the output a priori likelihood ratio for the APP decoder, which then calculates the output a posteriori likelihood ratio, denoted as $L_{\text {appost }}^{\text {Out }}$, for the coded symbols [59]. The output extrinsic information is calculated as $L_{\text {ext }}^{\text {Out }}=L_{\text {appost }}^{\text {Out }}-L_{a p}^{\text {Out }}$. After the interleaver $L_{\text {ext }}^{\text {Out }}$ becomes the input a priori likelihood

\footnotetext{
${ }^{11}$ We limit our descriptions of the channel estimator on the iterative procedures. Detailed descriptions of the calculation of LLRs can be found in [56].
} 
ratio $L_{a p}^{I n}$, which is used by the soft mapper to calculate the soft decision of the coded symbols for the cascaded 1D-FIR Wiener filtering. The re-estimated channel estimates are used for the soft I/O detector for the next iteration. In Sec. 5.3, we modify the estimator in Fig. 2.12 so that a similar frequency domain IDDCE (except that LLRs are not used for low complexity consideration) can be used for SC systems in time-varying frequency selective fading channels.

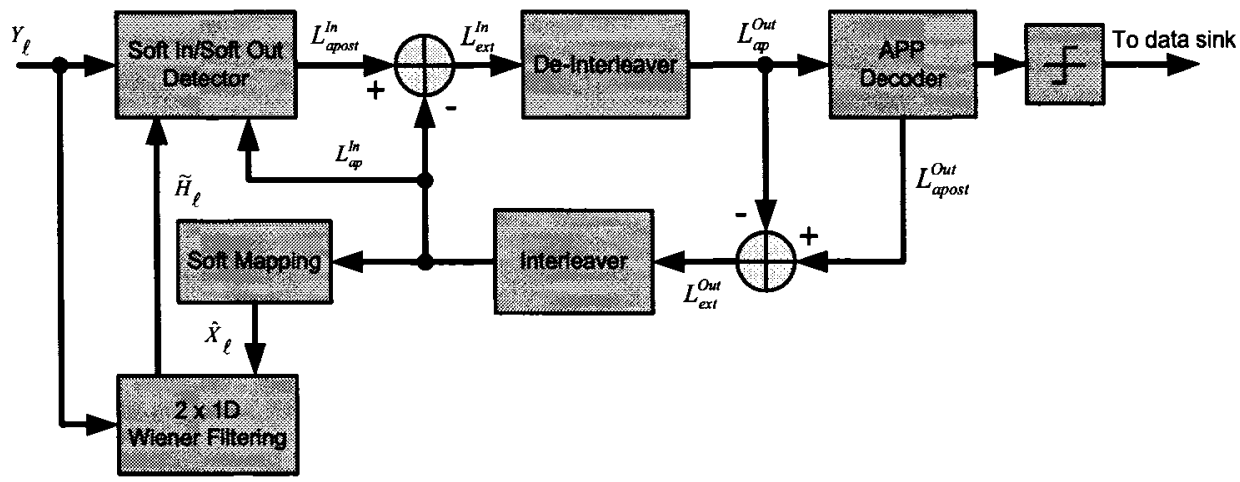

Figure 2.12: Iterative Decision-Directed Channel Estimator for OFDM Systems [56,79]

\subsection{Challenges of Designing Channel Estimation Techniques for Uplink SC Systems}

Table 2.5 depicts the summary of the individual requirement for the challenges of designing channel estimation techniques for future uplink SC systems in time-varying frequency selective fading channels. Note that HPA stands for high power amplifier and CE stands for channel estimation. It is almost impossible to satisfy all the requirements listed in Table 2.5 at the same time as there exist fundamental trade-offs among different requirements of the challenges, as shown in Fig. 2.13. Therefore, our approach to the design of channel estimation technique for uplink SC systems is based on the specific applications. For example, in the uplink, we need low cost terminal with long battery life for high mobility user terminals with broadband access. Hence the requirements of power backoff, implementation complexity and flexibility 
of spectrum allocations of pilot signals are prioritized.

Table 2.5: Summary of Challenges of Channel Estimation for Uplink SC Systems in Timevarying Frequency Selective Fading Channel

\begin{tabular}{|c|c|l|}
\hline Challenges & Requirements & \multicolumn{1}{|c|}{ Comments } \\
\hline $\begin{array}{c}\text { Spectrum Allocations } \\
\text { of Pilot Signals }\end{array}$ & Flexible & $\begin{array}{l}\text { Since single user can occupy full or partial, } \\
\text { or even discontinuous signal bandwidth, } \\
\text { pilot signals should be flexible to assign } \\
\text { to the corresponding signal bands. }\end{array}$ \\
\hline $\begin{array}{c}\text { Power Backoff } \\
\text { of HPA }\end{array}$ & Low & $\begin{array}{l}\text { Longer battery life and lower cost of user } \\
\text { terminal. }\end{array}$ \\
\hline Pilot Overhead & Low & Better spectral efficiency. \\
\hline MSE Performance & Small & Translates to better FER performance. \\
\hline $\begin{array}{c}\text { Support of Mobility } \\
\text { High }\end{array}$ & $\begin{array}{l}\text { High user mobility for wide area scenario } \\
\text { is a key requirement. }\end{array}$ \\
\hline $\begin{array}{c}\text { Implementation } \\
\text { Complexity }\end{array}$ & Low & $\begin{array}{l}\text { Lower cost and battery drain, smaller } \\
\text { processing delay. }\end{array}$ \\
\hline $\begin{array}{c}\text { Comparison with CE } \\
\text { for OFDM Systems }\end{array}$ & Comparable & $\begin{array}{l}\text { Should be comparable with that of OFDM } \\
\text { systems in terms of the above requirements. }\end{array}$ \\
\hline
\end{tabular}

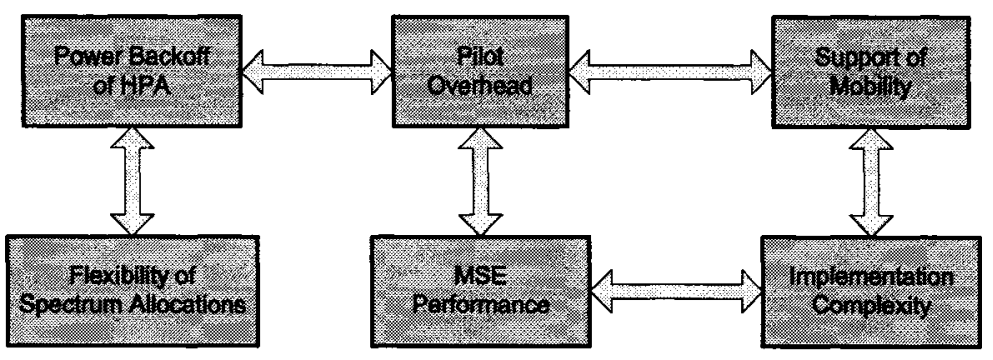

Figure 2.13: Illustration of Trade-offs among Different Requirements of the Challenges 


\section{Chapter 3}

\section{Effects of Channel Estimation Errors on BER Performance of SC Systems}

Before designing and developing FDM pilot schemes and channel estimation techniques for uplink SC systems, we analyze the effects of channel estimation errors (CEE) , characterized by mean square error (MSE), on BER performance of a frequency domain linear MMSE equalizer. Our motivation is to analytically study, in the frequency domain, the effects of CEE on the MSE of the equalizer output, which can be related to the BER performance using a Gaussian approximation. This analytical technique can be used to decouple the designing process of channel estimation from system performance. We first obtain an approximate expression for the BER performance in terms of the actual channel frequency response (CFR) and the MSE of the channel frequency estimates. We also use a semi-analytical approach to obtain the BER, by modeling the frequency channel estimates as the actual channel estimates plus a Gaussian random variable with zero mean and a variance of the averaged MSE of the channel estimates. Simulation results are used to verify our analytical and semi-analytical results. 


\subsection{Introduction}

Assuming the residual error at the equalizer output is Gaussian, with zero mean and with a variance of $J_{o}$, the BER performance of an uncoded QPSK system with perfect channel estimates, i.e. the channel is known at the receiver, can be obtained by using

$$
\mathrm{BER}=Q\left(\sqrt{\frac{1-J_{o}}{J_{o}}}\right)
$$

where $Q(x)$ is the $Q$-function defined as $Q(x)=\frac{1}{\sqrt{2 \pi}} \int_{x}^{\infty} e^{-t^{2} / 2} d t, J_{o}$ is the MMSE of the equalizer output when the channel is known and the data symbols have zero mean and unit variance. In particular, for a frequency domain symbol-rate-sampled linear MMSE (LMMSE) equalizer in multipath channels, $J_{o}$ can be found, in the frequency domain, as in (2.27), rewritten here for convenience as

$$
J_{o}=\frac{\sigma^{2}}{M} \sum_{\ell=0}^{M-1} \frac{1}{\left|H_{\ell}\right|^{2}+\sigma^{2}}
$$

where $M$ is the number of data symbols, $H_{\ell}$ is the CFR at the $\ell$ th frequency and $\sigma^{2}$ is the noise variance. Previous work on the BER estimation for an uncoded BPSK SC system using frequency domain equalizer with imperfect channel estimates is found in [76]. The authors derived the probability density function (PDF) of the frequency LMMSE equalizer taps, assuming frequency domain LS channel estimation. With a Gaussian approximation for the residual ISI and using the PDF for the frequency domain LMMSE equalizer taps with LS frequency channel estimates, an analytical result for BER performance of an uncoded BPSK system with LS channel estimates is obtained, requiring numerical integrations. Note that in practice the BER is usually obtained by actually simulating the complete system with a channel estimation subsystem, which implies a longer simulation time.

In this chapter, we analyze the effects of channel estimation errors, characterized by MSE, on BER performance of a frequency domain LMMSE equalizer. Our goal is to estimate the BER performance for an uncoded QPSK system with CEE, given the averaged MSE of the channel frequency estimates and the actual CFR. The first 
approach is to analytically obtain an expression for the BER performance in terms of the actual CFR and the MSE of the CFR estimates. This analytical approach assumes the knowledge of the second order statistic (or MSE) of the CFR estimates and uses the MSE of the CFR estimates to calculate the MSE of the equalizer output, which is then used to estimate the BER performance. The realizations of the CFR estimates are not required. The second approach (called the semi-analytical approach) is to obtain the BER by direct substitution of the simulated realizations of the CFR estimates, modeled as the sum of the actual CFR and the channel estimation error (generated by a Gaussian random variable), into the calculation of the MSE of the equalizer output. The semi-analytical approach assumes not only the knowledge of the second order statistic of the CFR estimates but also the realizations of the simulated CFR estimates, which are used to obtain the MSE of the equalizer output by using Monte Carlo simulation. The difference between the analytical and the semianalytical approach is the way of how to obtain the MSE of the equalizer output.

\subsection{Analytical Approach}

Our approach is to first derive the MSE of the LMMSE equalizer output with CEE. We assume that the averaged MSE of the channel estimates over all frequencies is known and that the residual ISI can be approximated as a Gaussian random variable so that (3.1) and the following equation

$$
\mathrm{BER}=Q\left(\sqrt{S N R_{e q z}}\right)
$$

can be used to estimate the BER of an uncoded QPSK system with imperfect channel estimates, where $Q(x)$ is the $Q$-function and $S N R_{\text {eqz }}$ denotes the SNR at the equalizer output. Notice that using (3.1), we need only the MSE of the equalizer output, while using (3.3) we need to calculate $S N R_{e q z}$ from the $m$ th data output of the equalizer. 


\subsubsection{Calculation of $S N R_{e q z}$}

The equalizer output for the $m$ th data symbol $a_{m}$ can be written as

$$
\tilde{a}_{m}=\kappa a_{m}+e_{m}
$$

where $\kappa$ is a gain variable related to the channel impulse response and the equalizer taps and $e_{m}$ is the sum of the residual inter-symbol interference (ISI) and the equalizer output noise, which can be written as

$$
e_{m}=\epsilon_{m}+(1-\kappa) a_{m}
$$

where $\epsilon_{m} \triangleq \tilde{a}_{m}-a_{m}$. From (3.4), SNR $R_{e q z}$ can be found as

$$
\begin{aligned}
S N R_{e q z} & =\frac{E\left\{\left|\kappa a_{m}\right|^{2}\right\}}{E\left\{\left|e_{m}\right|^{2}\right\}} \\
& =\frac{E\left\{|\kappa|^{2}\right\} E\left\{\left|a_{m}\right|^{2}\right\}}{E\left\{\left|\epsilon_{m}\right|^{2}\right\}+E\left\{|1-\kappa|^{2}\right\}+2 \Re\left\{E\{1-\kappa\}\left[E\left\{\epsilon_{m} \tilde{a}_{m}^{*}\right\}-E\left\{\left|\epsilon_{m}\right|^{2}\right\}\right]\right\}} \\
& =\frac{E\left\{|\kappa|^{2}\right\}}{E\left\{\left|\epsilon_{m}\right|^{2}\right\}[2 E\{\kappa\}-1]+E\left\{|1-\kappa|^{2}\right\}}
\end{aligned}
$$

where we make the following assumptions:

1. $E\left\{\left|a_{m}\right|^{2}\right\}=1$

2. $E\left\{|\kappa|^{2}\left|a_{m}\right|^{2}\right\}=E\left\{|\kappa|^{2}\right\} E\left\{\left|a_{m}\right|^{2}\right\}$

3. $E\left\{\epsilon_{m} \tilde{a}_{m}^{*}\right\}=0$

4. $E\left\{\kappa \epsilon_{m} \tilde{a}_{m}\right\}=E\{\kappa\} E\left\{\epsilon_{m} \tilde{a}_{m}\right\}$

where the second assumption comes from the orthogonal condition in deriving MMSE equalizer [35, pp. 624] and $E\left\{\left|\epsilon_{m}\right|^{2}\right\}$ is the MSE of the equalizer output. From (3.6), it is obvious that in order to calculate $S N R_{e q z}$ we need to find $E\left\{\left|\epsilon_{m}\right|^{2}\right\}, E\left\{|\kappa|^{2}\right\}$ and $E\{\kappa\}$. Note that the last assumption implies that $\kappa$ is independent of $\epsilon_{m} \tilde{a}_{m}$. 


\subsubsection{Determination of $J_{A} \triangleq E\left\{\left|\epsilon_{m}\right|^{2}\right\}$}

Define the CFR with CEE at the $\ell$ th frequency as

$$
\tilde{H}_{\ell}=H_{\ell}+\Delta H_{\ell}
$$

where $\Delta H_{\ell}$ is the channel estimation error at the $\ell$ th frequency. By substituting

$$
C_{\ell}=\frac{\tilde{H}_{\ell}^{*}}{\left|\tilde{H}_{\ell}\right|^{2}+\sigma^{2}}
$$

into

$$
J=\frac{1}{M} \sum_{\ell=0}^{M-1}\left|C_{\ell} H_{\ell}-1\right|^{2}+\frac{\sigma^{2}}{M} \sum_{\ell=0}^{M-1}\left|C_{\ell}\right|^{2}
$$

and using

$$
H_{\ell}=\tilde{H}_{\ell}-\Delta H_{\ell}
$$

the MSE of the equalizer output using the imperfect channel frequency estimate $\tilde{H}_{\ell}$ can be found as

$$
\begin{aligned}
J_{A} & =\frac{1}{M} \sum_{\ell=0}^{M-1}\left|\frac{\tilde{H}_{\ell}^{*}}{\left|\tilde{H}_{\ell}\right|^{2}+\sigma^{2}}\left(\tilde{H}_{\ell}-\Delta H_{\ell}\right)-1\right|^{2}+\frac{\sigma^{2}}{M} \sum_{\ell=0}^{M-1}\left|\frac{\tilde{H}_{\ell}^{*}}{\left|\tilde{H}_{\ell}\right|^{2}+\sigma^{2}}\right|^{2} \\
& =\frac{\sigma^{2}}{M} \sum_{\ell=0}^{M-1} \frac{1}{\left|\tilde{H}_{\ell}\right|^{2}+\sigma^{2}}+\frac{1}{M} \sum_{\ell=0}^{M-1}\left[2 \Re\left\{\frac{\sigma^{2} \tilde{H}_{\ell}^{*} \Delta H_{\ell}}{\left(\left|\tilde{H}_{\ell}\right|^{2}+\sigma^{2}\right)^{2}}\right\}+\left|\frac{\tilde{H}_{\ell}^{*} \Delta H_{\ell}}{\left|\tilde{H}_{\ell}\right|^{2}+\sigma^{2}}\right|^{2}\right]
\end{aligned}
$$

where $J_{A} \triangleq E\left\{\left|\epsilon_{m}\right|^{2}\right\}$ and $\Re\{z\}$ represents the real part of a complex number $z$. Note that the detailed derivation of (3.11) is shown in Appendix A.1. To further simplify (3.11), we make the following assumptions:

1. $\sum_{\ell=0}^{M-1} \frac{1}{\left|\tilde{H}_{\ell}\right|^{2}+\sigma^{2}} \approx \sum_{\ell=0}^{M-1} \frac{1}{\left|H_{\ell}\right|^{2}+\sigma^{2}} \quad$ (Assumption 1a)

2. $\sum_{\ell=0}^{M-1} \frac{\left|\tilde{H}_{\ell}\right|^{2}}{\left(\left|\tilde{H}_{\ell}\right|^{2}+\sigma^{2}\right)^{2}} \approx \sum_{\ell=0}^{M-1} \frac{\left|H_{\ell}\right|^{2}}{\left(\left|H_{\ell}\right|^{2}+\sigma^{2}\right)^{2}} \quad$ (Assumption 1b)

3. $E\left\{\tilde{H}_{\ell}^{*} \Delta H_{\ell}\right\}=0, \quad \ell=0,1, \cdots, M-1 \quad$ (Assumption 2)

4. $E\left\{\left|\Delta H_{\ell}\right|^{2}\right\}=\sigma_{\Delta H}^{2}, \quad \ell=0,1, \cdots, M-1 . \quad$ (Assumption 3) 
Assumption 2 comes from the orthogonal projection theorem used when deriving the LMMSE channel estimator [71]. Assumption 3 assumes that the MSE of the channel estimates at different frequencies are constant. Taking the expectation with respect to $\Delta H_{\ell},(3.11)$ can then be simplified as

$$
\tilde{J}_{A} \approx \frac{\sigma^{2}}{M} \sum_{\ell=0}^{M-1} \frac{1}{\left|H_{\ell}\right|^{2}+\sigma^{2}}+\frac{\sigma_{\Delta H}^{2}}{M} \sum_{\ell=0}^{M-1} \frac{\left|H_{\ell}\right|^{2}}{\left(\left|H_{\ell}\right|^{2}+\sigma^{2}\right)^{2}}
$$

where $\tilde{J}_{A}$ represents the expected value of $J_{A}$. Notice that the first term in $(3.12)$ is just the MSE of the equalizer output when the channel is perfectly known (see (3.2)). The second term in (3.12) is the increase in MSE due to channel estimation error. Note that in our derivation, the channel estimation error $\Delta H_{\ell}$ is not necessarily a Gaussian random variable, as long as it satisfies Assumption 2 and Assumption 3.

\subsubsection{Determination of $E\{\kappa\}$ and $E\left\{|\kappa|^{2}\right\}$}

Using (3.8) and (3.10), $\kappa$ can be calculated as

$$
\begin{aligned}
\kappa & =\frac{1}{M} \sum_{\ell=0}^{M-1} H_{\ell} C_{\ell} \\
& =\frac{1}{M} \sum_{\ell=0}^{M-1} \frac{\left|\tilde{H}_{\ell}\right|^{2}-\Delta H_{\ell} \tilde{H}_{\ell}^{*}}{\left|\tilde{H}_{\ell}\right|^{2}+\sigma^{2}}
\end{aligned}
$$

Taking the expectation with respect to $\Delta H_{\ell}, E\{\kappa\}$ can be found as

$$
E\{\kappa\} \approx \frac{1}{M} \sum_{\ell=0}^{M-1} \frac{\left|H_{\ell}\right|^{2}}{\left|H_{\ell}\right|^{2}+\sigma^{2}}
$$

where in addition to Assumption 2, we also assume

- $\sum_{\ell=0}^{M-1} \frac{\left|\tilde{H}_{\ell}\right|^{2}}{\left|\tilde{H}_{\ell}\right|^{2}+\sigma^{2}} \approx \sum_{\ell=0}^{M-1} \frac{\left|H_{\ell}\right|^{2}}{\left|H_{\ell}\right|^{2}+\sigma^{2}}$. (Assumption 1c) 
We next find $E\{|\kappa|\}^{2}$, given as

$$
\begin{aligned}
& E\left\{|\kappa|^{2}\right\}=E\left\{\left|\frac{1}{M} \sum_{\ell=0}^{M-1} \frac{\left|\tilde{H}_{\ell}\right|^{2}-\Delta H_{\ell} \tilde{H}_{\ell}^{*}}{\left|\tilde{H}_{\ell}\right|^{2}+\sigma^{2}}\right|^{2}\right\} \\
& \approx \frac{1}{M^{2}} \sum_{\ell=0}^{M-1} \frac{\left|H_{\ell}\right|^{2}\left(\left|H_{\ell}\right|^{2}+\sigma_{\Delta H}^{2}\right)}{\left(\left|H_{\ell}\right|^{2}+\sigma^{2}\right)^{2}} \\
& +\frac{1}{M^{2}} \sum_{\substack{\ell=0 \\
\ell \neq k}}^{M-1} \sum_{\substack{k=0 \\
k \neq \ell}}^{M-1} \frac{\left|H_{\ell}\right|^{2}\left|H_{k}\right|^{2}}{\left(\left|H_{\ell}\right|^{2}+\sigma^{2}\right)\left(\left|H_{k}\right|^{2}+\sigma^{2}\right)}
\end{aligned}
$$

where in addition to using Assumption 1b, 1c and 2, we also assume:

1. $\sum_{\ell=0}^{M-1} \frac{\left|\tilde{H}_{\ell}\right|^{4}}{\left(\left|\tilde{H}_{\ell}\right|^{2}+\sigma^{2}\right)^{2}} \approx \sum_{\ell=0}^{M-1} \frac{\left|H_{\ell}\right|^{4}}{\left(\left|H_{\ell}\right|^{2}+\sigma^{2}\right)^{2}} \quad$ (Assumption 1d)

2. $\sum_{\substack{\ell=0 \\ \ell \neq k}}^{M-1} \sum_{\substack{k=0 \\ k \neq \ell}}^{M-1} \frac{\left|\tilde{H}_{\ell}\right|^{2}\left|\tilde{H}_{k}\right|^{2}}{\left(\left|\tilde{H}_{\ell}\right|^{2}+\sigma^{2}\right)\left(\left|\tilde{H}_{k}\right|^{2}+\sigma^{2}\right)} \approx \sum_{\substack{\ell=0 \\ \ell \neq k}}^{M-1} \sum_{\substack{k=0 \\ k \neq \ell}}^{M-1} \frac{\left|H_{\ell}\right|^{2}\left|H_{k}\right|^{2}}{\left(\left|H_{\ell}\right|^{2}+\sigma^{2}\right)\left(\left|H_{k}\right|^{2}+\sigma^{2}\right)}$ (Assumption 1e)

3. $E\left\{\Delta H_{\ell} \Delta H_{k}^{*}\right\}=0, \quad k \neq \ell \quad$ (Assumption 4)

Note that the detailed derivation of (3.15) is included in Appendix A.2. Assumption 4 states that the channel estimation errors at different frequencies are uncorrelated. Using (3.14) and (3.15), we can also find $E\left\{|1-\kappa|^{2}\right\}$, given as

$$
E\left\{|1-\kappa|^{2}\right\}=1-2 E\{\kappa\}+E\{|\kappa|\}^{2}
$$

Substituting (3.12), (3.14), (3.15) and (3.16) into (3.6), we obtain the approximated SNR of the equalizer output with imperfect channel estimate, given as

$$
S N R_{e q z} \approx \frac{E\left\{|\kappa|^{2}\right\}}{(2 E\{\kappa\}-1) \tilde{J}_{A}+E\left\{|\kappa|^{2}\right\}-2 E\{\kappa\}+1} .
$$

Using the Gaussian approximation for the residual ISI of the equalizer output, the estimated BER can then be found by substituting (3.17) into (3.3) or (3.12) into (3.1). Notice that using (3.3) requires not only the MSE of the equalizer but also the first and the second order statistics of the gain variable $\kappa$. 


\subsection{Semi-Analytical Approach}

Another approach to estimating the BER is to first model the channel estimation error (CEE) $\Delta H_{\ell}$ as a random variable with known probability density function (PDF) and substitute (3.7) into (3.9) for direct evaluation of the MSE of the equalizer output, given as

$$
\tilde{J}_{S}=\frac{1}{M} \sum_{\ell=0}^{M-1} E\left\{\left|\frac{\tilde{H}_{\ell}^{*}}{\left|\tilde{H}_{\ell}\right|^{2}+\sigma^{2}} H_{\ell}-1\right|^{2}+\frac{\sigma^{2}}{M} \sum_{\ell=0}^{M-1}\left|\frac{\tilde{H}_{\ell}^{*}}{\left|\tilde{H}_{\ell}\right|^{2}+\sigma^{2}}\right|^{2}\right\}
$$

where $\tilde{J}_{S} \triangleq E\left\{\left|a_{m}-\tilde{a}_{m}\right|^{2}\right\}$ is the MSE of the equalizer output using a semi-analytical approach and $\tilde{H}_{\ell}=H_{\ell}+\Delta H_{\ell} . \tilde{J}_{S}$ is then substituted into (3.1) to estimate the BER. This approach is semi-analytical since we have an expectation operation $E\{\bullet\}$ with respect to the CEE $\Delta H_{\ell}$ in the equation. Assuming that a PDF for $\Delta H_{\ell}$ is known, Monte Carlo simulation is used to obtain $\tilde{J}_{S}$ for a particular realization of the actual channel frequency response (CFR), by simulating the realizations of the CEE $\Delta H_{\ell}$ and substituting $\tilde{H}_{\ell}=H_{\ell}+\Delta H_{\ell}$ into (3.18). Note that the difference between (3.18) and (3.12) is that for a particular realization of the actual CFR, Monte Carlo simulation is not required for (3.12).

The key to this approach is the accuracy of the statistical modeling of the channel estimation errors. We model both the FFT and Wiener channel estimation error $\Delta H_{\ell}$ as an independent, zero mean Gaussian random variable with variance being the time-averaged MSE of the channel frequency estimates, given as

$$
\sigma_{\Delta H}^{2}=\frac{1}{M} \sum_{\ell=0}^{M-1}\left|\Delta H_{\ell}\right|^{2}
$$

where $M$ is the number of data symbols per block (or per OFDM symbol). We first evaluate the accuracy of the modeling of $\Delta H_{\ell}$ by comparing the cumulative density function (CDF) of $\Delta H_{\ell}$, obtained by simulation, with that of a Gaussian random variable with zero mean and a variance of $\sigma_{\Delta H}^{2}$. Fig. 3.1 shows the CDF of the real part of $\Delta H_{\ell}$, denoted as $F_{\Re\left\{\Delta \mathbf{H}_{\ell}\right\}}(x)$, and that of a Gaussian random variable, 


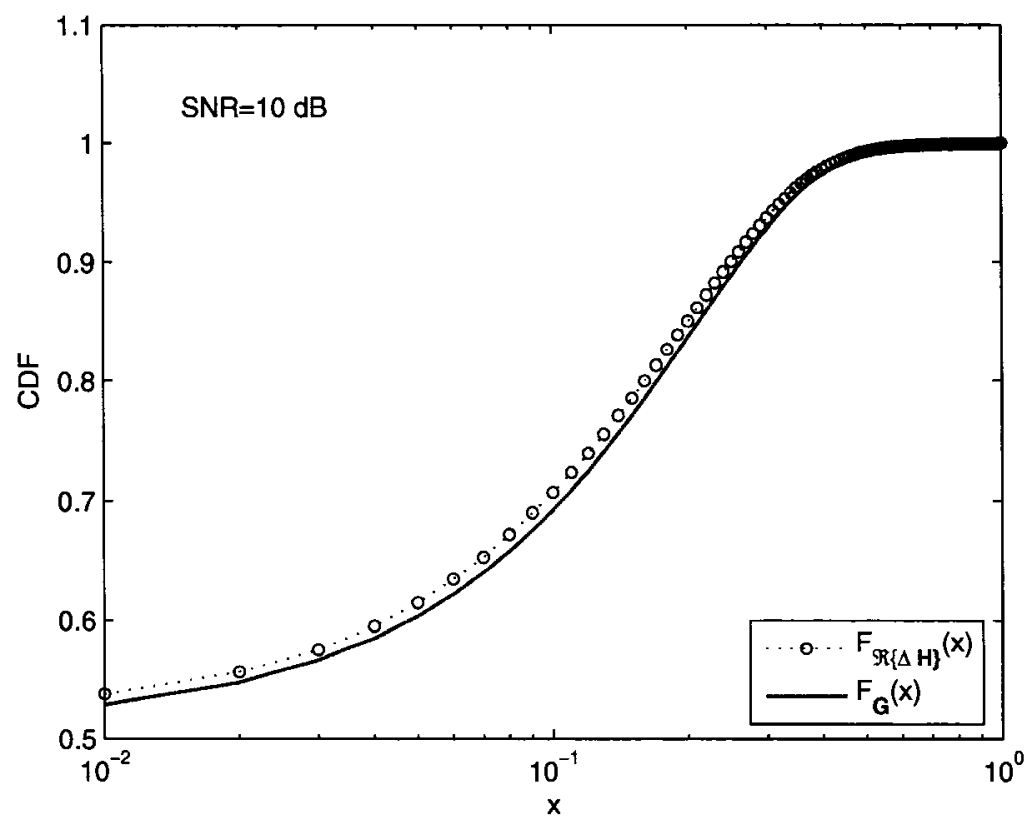

Figure 3.1: Comparison of the CDF of the Real Part of $\Delta H_{\ell}$ from an FFT Estimator $(M=416$ and $N=52)$ and that of a Gaussian Random Variable with Zero Mean and a Variance of $\sigma_{\Delta H}^{2}=0.088$ at $\mathrm{SNR}=10 \mathrm{~dB}$

denoted as $F_{\mathbf{G}}(x)$. The channel estimator used is the FFT estimator ( $M=416$ and $N=52$ ) with $\sigma_{\Delta H}^{2}=0.088$ (see Fig. 3.5) at SNR $=10 \mathrm{~dB}$ in WINNER C2 channel with no mobility (see Table 2.3). Note that $N$ is the number of pilots per block. 832000 samples and 201 bins (ranging from -1 to 1) are used to obtain the estimated CDF of $\Delta H_{\ell}$. Observe that the two CDFs almost coincide with each other.

Fig. 3.2 shows the CDF for the same parameters as that of Fig. 3.1, except that $\mathrm{SNR}=20 \mathrm{~dB}$ and $\sigma_{\Delta H_{\ell}}^{2}=0.018$ (see Fig. 3.5). In this case, the two CDFs do not match, which results in a slightly off estimation of the BER performance at SNR = $20 \mathrm{~dB}$. The mismatch comes from the fact that the interpolation error of the FFT channel estimator at high SNR, which are likely correlated for different frequencies, dominates the white noise. Moreover, at high SNR, the edge effect of the FFT channel estimator [70] is more significant such that Assumption 3 is inaccurate.

The plots of $F_{\Re\left\{\Delta \mathbf{H}_{\ell}\right\}}(x)$ and $F_{\mathrm{G}}(x)$ for Wiener channel estimation errors with 


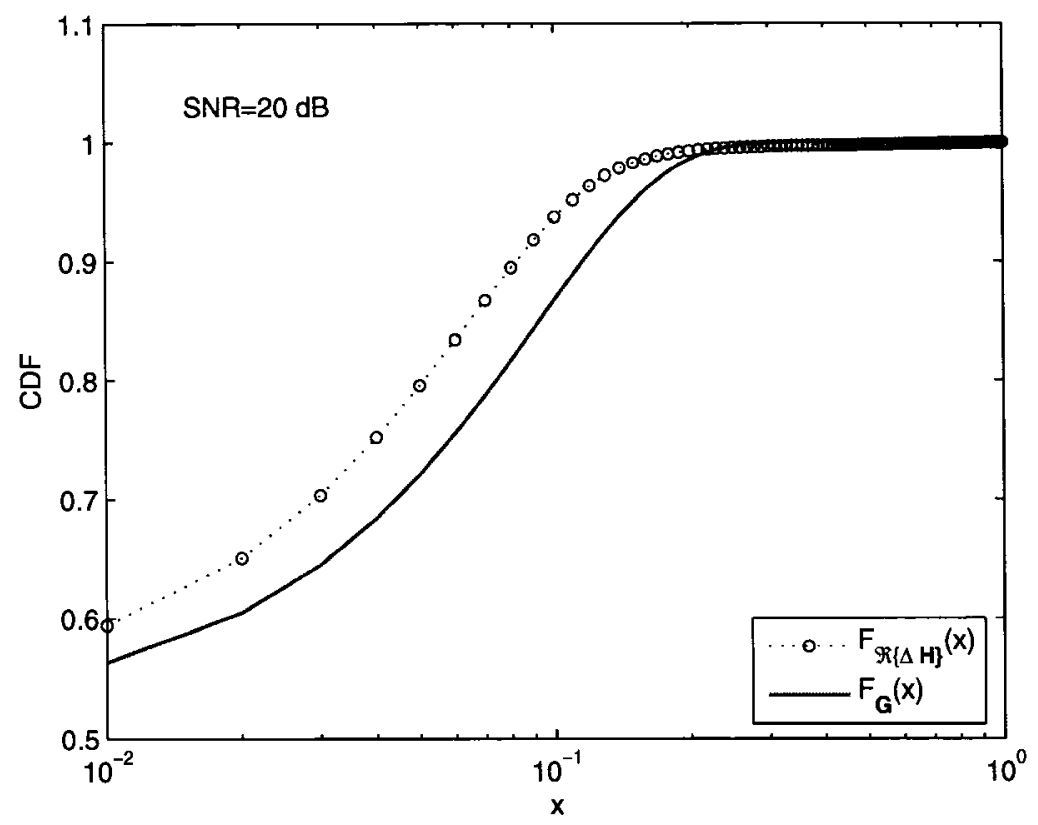

Figure 3.2: Comparison of the CDF of the Real Part of $\Delta H_{\ell}$ from an FFT Estimator $(M=416$ and $N=52)$ and that of a Gaussian Random Variable with Zero Mean and a Variance of $\sigma_{\Delta H}^{2}=0.018$ at $\mathrm{SNR}=20 \mathrm{~dB}$

$\mathrm{SNR}=10 \mathrm{~dB}$ and $\mathrm{SNR}=20 \mathrm{~dB}$ are shown in Fig. 3.3 and Fig. 3.4, respectively. In both cases, $F_{\Re\left\{\Delta \mathbf{H}_{\ell}\right\}}(x)$ and $F_{\mathbf{G}}(x)$ almost coincide with each other. Note that $\sigma_{\Delta H}^{2}$ at $\mathrm{SNR}=10 \mathrm{~dB}$ and $20 \mathrm{~dB}$ are obtained from Fig. 3.6. From Fig. 3.1 to Fig. 3.4, we can conclude that the modeling of $\Delta H_{\ell}$ as a zero mean Gaussian random variable with variance of $\sigma_{\Delta H}^{2}$ is a reasonable choice, at least for FFT and Wiener Estimators in WINNER C2 channel. The disadvantage of this approach is that different estimation error may require different statistical models although the Gaussian approximation seems to be a reasonable choice. By replacing $J_{o}$ in (3.1) with the time average of $\tilde{J}_{S}$, the estimated BER for an uncoded QPSK system can be estimated as,

$$
\mathrm{BER} \approx Q\left(\sqrt{\frac{1-\tilde{J}_{S}}{\tilde{J}_{S}}}\right)
$$

Note that we use (3.20) instead of an equation similar to (3.17) for simplicity. We next verify our analytical and semi-analytical approaches using computer simulation. 


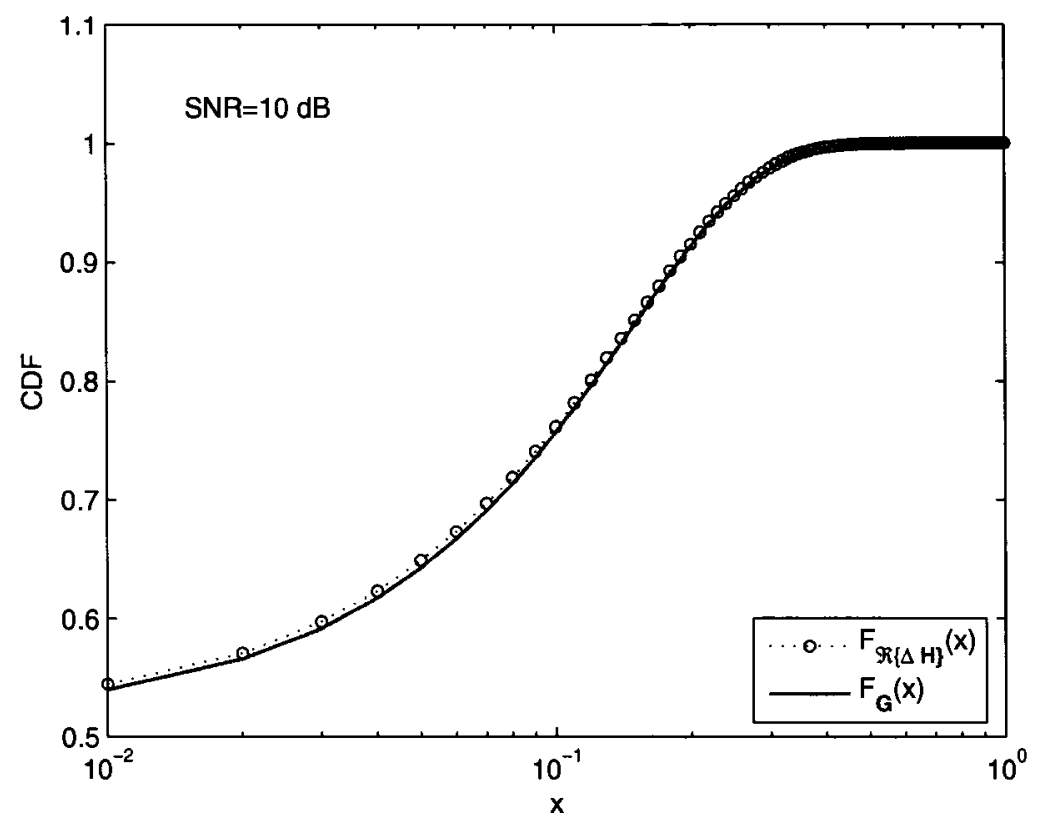

Figure 3.3: Comparison of the CDF of the Real Part of $\Delta H_{\ell}$ from a Wiener Estimator ( $M=416$ and $N=104$ ) and that of a Gaussian Random Variable with Zero Mean and a Variance of $\sigma_{\Delta H}^{2}=0.045$ at $\mathrm{SNR}=10 \mathrm{~dB}$

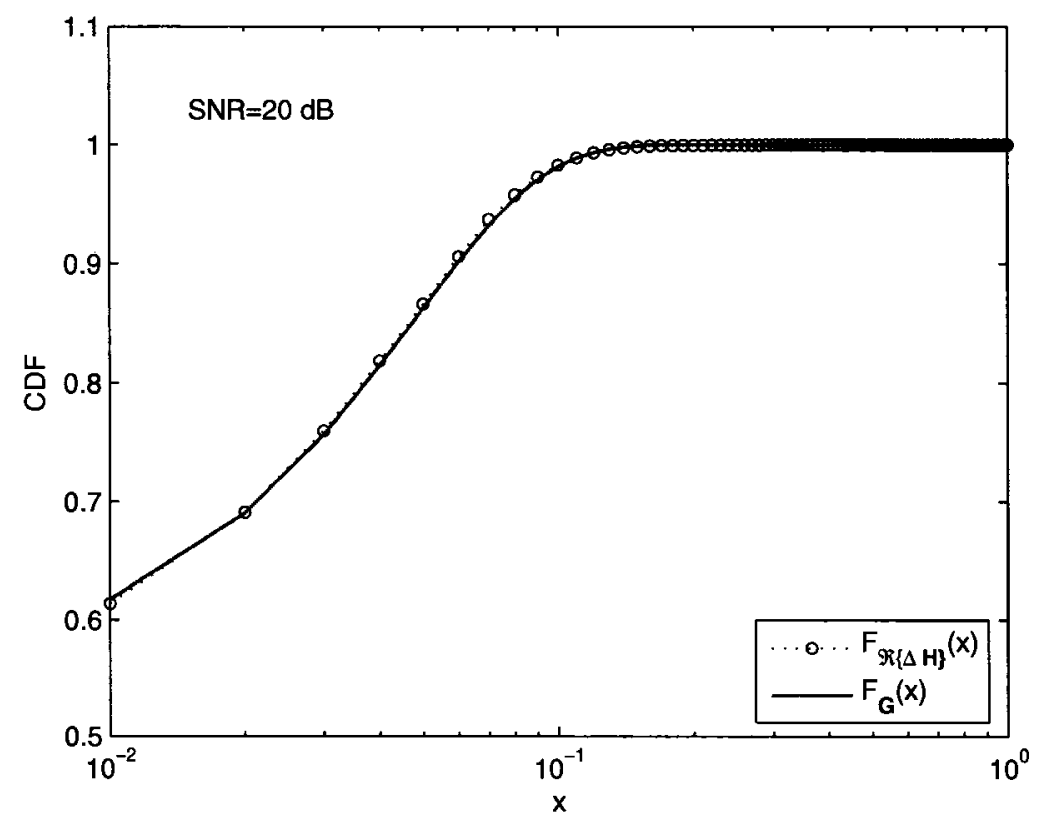

Figure 3.4: Comparison of the CDF of the Real Part of $\Delta H_{\ell}$ from a Wiener Estimator $(M=416$ and $N=104)$ and that of a Gaussian Random Variable with Zero Mean and a Variance of $\sigma_{\Delta H}^{2}=0.0051$ at $\mathrm{SNR}=20 \mathrm{~dB}$ 


\subsection{Simulation Results}

The simulation system parameter used is Parameter Set 2-A, except that no channel coding is used, the channel is static and each frame contains one block. The channel estimators used for evaluation are the FFT and the Wiener estimator using FET pilots (to be discussed in Chapter 5). 2000 channel realizations are used to obtain the simulation results.

\subsubsection{MSE of Equalizer Output}

Fig. 3.5 and Fig. 3.6 depict the comparison of the simulation and analytical results for obtaining the MSE of the equalizer output $E\left\{\left|a_{m}-\tilde{a}_{m}\right|^{2}\right\}$ using the FFT and Wiener channel estimators, respectively. (3.12) and (3.18) are used to obtain the analytical and semi-analytical results, respectively. The time-averaged MSE of the corresponding channel estimate is also included for reference. We can see that the analytical and semi-analytical approaches produce the same MSE of the equalizer

output as that from the simulation. A discrepancy is observed for high SNR $(>17$ $\mathrm{dB}$ ) in Fig. 3.5, resulting from the assumption that the MSE of the channel estimates is constant over all frequencies. At high SNR, the interpolation error of the FFT channel estimator dominates the effects of the noise such that the assumption of a constant time-averaged MSE for all frequencies is invalid.

\subsubsection{BER Estimation}

Fig. 3.7 and Fig. 3.8 show the performance of the uncoded BER estimation approaches using FFT and Wiener estimators with FET pilots in WINNER C2 channel (no mobility), respectively. The simulation and analytical BER performance for systems with known channels are included for reference. First consider Fig. 3.7 for the FFT estimator. Using (3.1), both analytical and semi-analytical approaches produce accurate BER estimations for SNR $<17 \mathrm{~dB}$. A discrepancy occurs in the high SNR region, e.g. $S N R \geq 17 \mathrm{~dB}$, resulting from the inaccurate modeling of channel estima- 


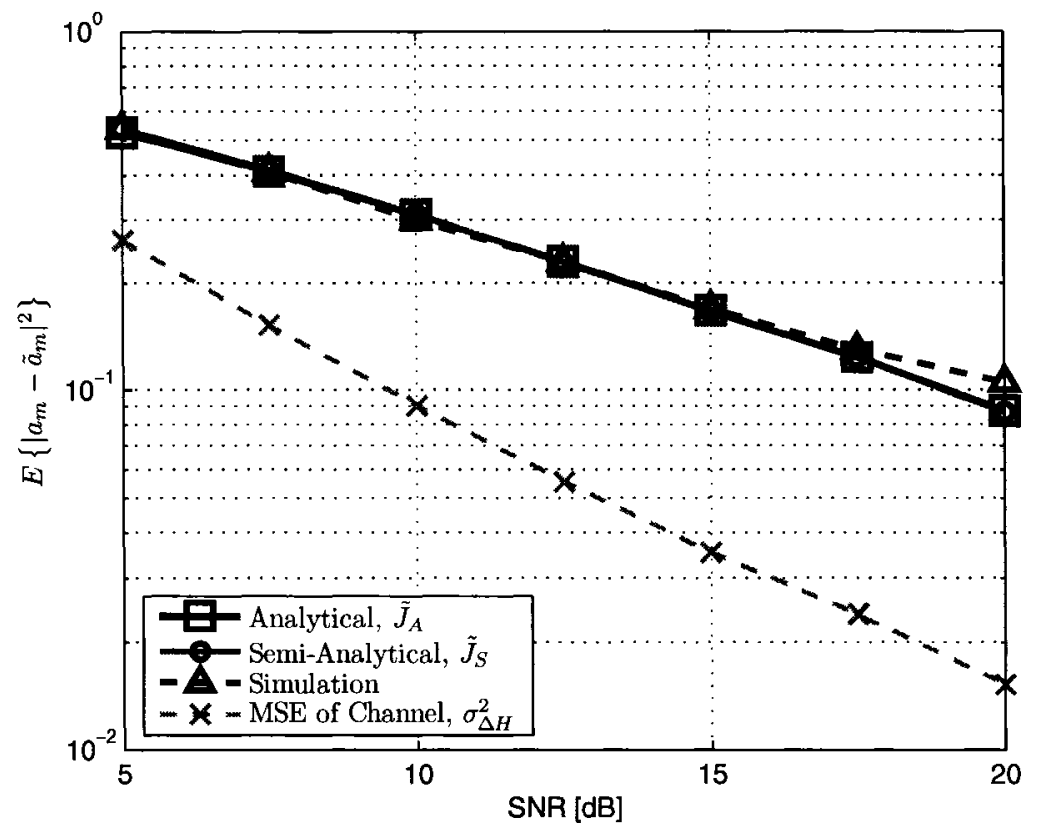

Figure 3.5: Comparison of Simulated and Analytical Results for MSE of Equalizer Output with Channel Estimation Errors using FFT Channel Estimator

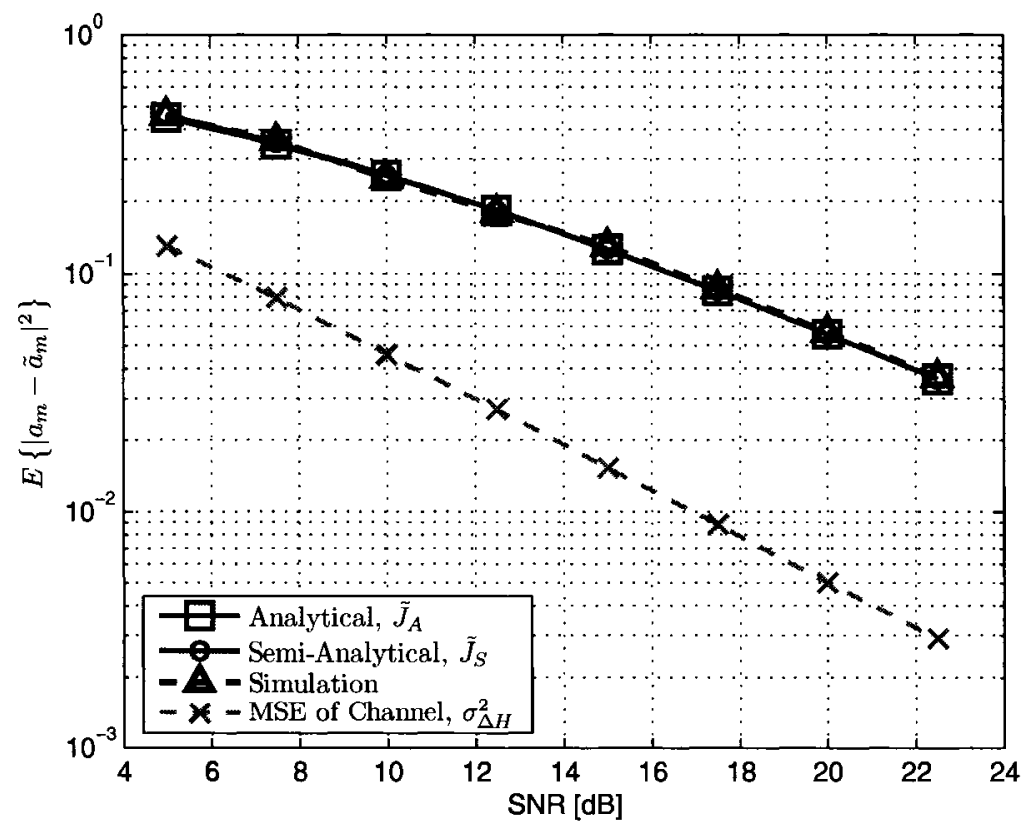

Figure 3.6: Comparison of Simulated and Analytical Results for MSE of Equalizer Output with Channel Estimation Errors using Wiener Channel Estimator 


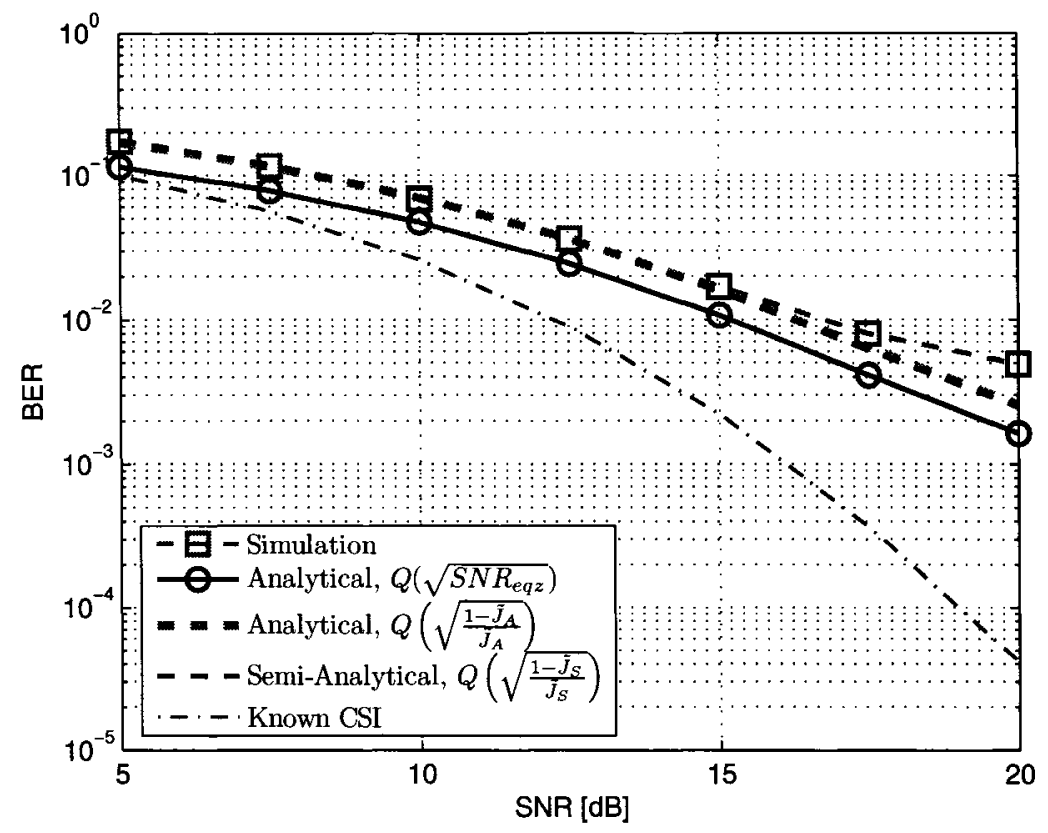

Figure 3.7: Evaluation of BER Estimation Approaches for an Uncoded QPSK System using FFT Channel Estimator with FET Pilots in WINNER C2 Channel (no mobility)

tion error at high SNR, as shown in Fig. 3.2 and Fig. 3.5. Using (3.3), the estimated BER curve is about $1 \mathrm{~dB}$ off the simulated BER curve. The difference can be explained by Assumption 4 for deriving (3.15), which is used by (3.3). This implies that at high SNR, the CEE from an FFT estimator is correlated at different frequencies, i.e. $E\left\{\Delta H_{\ell} \Delta H_{k}^{*}\right\} \neq 0$ for $k \neq \ell$. Moreover, the use of (3.1) and (3.3) to estimate the BER relies on the Gaussian approximation of the equalizer output noise.

Similar results are obtained for the Wiener channel estimator for QPSK, as shown in Fig. 3.8, except that the estimated BER curve using (3.3) is only about $0.5 \mathrm{~dB}$ away from the simulated BER curve, implying that the correlation of the Wiener channel estimation error at different frequencies is smaller than that of an FFT estimator. Moreover, there is no discrepancy between the simulation results and the analytical results using (3.1) at high $\mathrm{SNR}$, such as $\mathrm{SNR}=20 \mathrm{~dB}$, resulting from an accurate modeling of CEE at $\mathrm{SNR}=20 \mathrm{~dB}$, as shown in Fig. 3.4. Both analytical and semianalytical approaches are also applicable for uncoded 16-QAM SC systems, as shown 


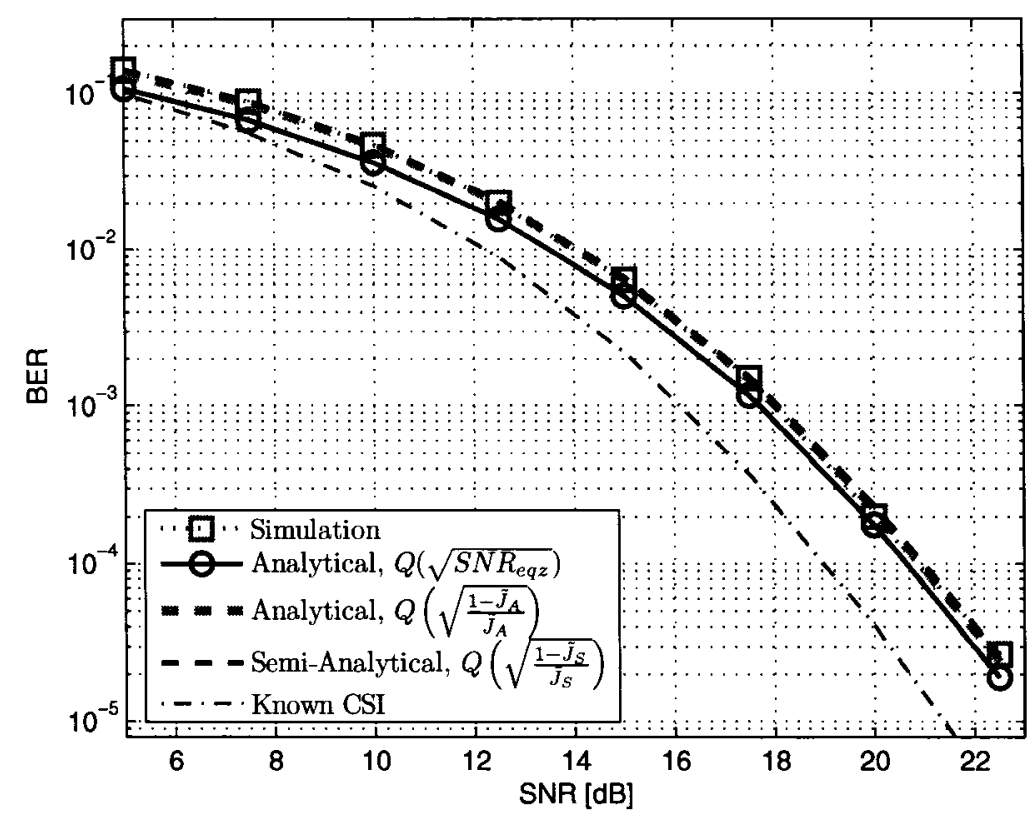

Figure 3.8: Evaluation of BER Estimation Approaches for Uncoded QPSK SC Systems using Wiener Channel Estimator with FET Pilots in WINNER C2 Channel (no mobility)

in Fig. 3.9. The formula used to obtain the analytical and semi-analytical BER for 16-QAM systems is given as [104],

$$
\mathrm{BER} \approx \frac{3}{8} \operatorname{erfc}\left(\sqrt{\frac{1}{10} S N R}\right)+\frac{1}{4} \operatorname{erfc}\left(\sqrt{\frac{9}{10} S N R}\right)+\frac{1}{8} \operatorname{erfc}\left(\sqrt{\frac{5}{2} S N R}\right)
$$

where $\operatorname{erfc}(x)=\frac{2}{\sqrt{\pi}} \int_{x}^{\infty} e^{-t^{2}} d t$ is the complementary error function and $S N R$ is the signal to noise ratio, which can be $S N R_{e q z}, \frac{1-\tilde{J}_{A}}{\tilde{J}_{A}}$ or $\frac{1-\tilde{J}_{S}}{\tilde{J}_{S}}$, depending on the particular approach used.

Fig. 3.10 illustrates the application of the CEE model for the semi-analytical approach for a coded QPSK system, where 'CE' stands for channel estimation. In this case, we assume the simulated CFR estimates are the estimates from the channel estimator. Parameter Set 2-B (see Table 2.3 and 2.4) is used in the simulation. The channel estimator used is the cascaded $2 \times 1 \mathrm{D}$ Wiener channel estimator. We can see that the semi-analytical approach produces consistent BER and FER results with that of the simulation results. 


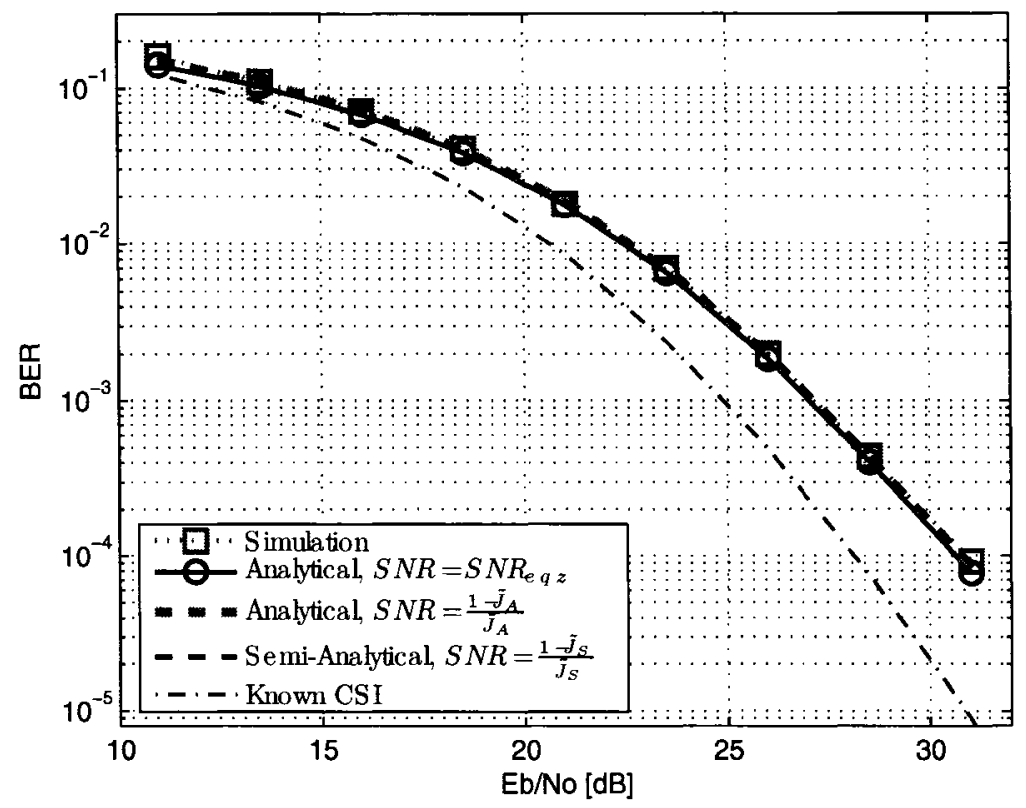

Figure 3.9: Evaluation of BER Estimation Approaches for Uncoded 16-QAM SC Systems using Wiener Channel Estimator with FET Pilots in WINNER C2 Channel (no mobility)

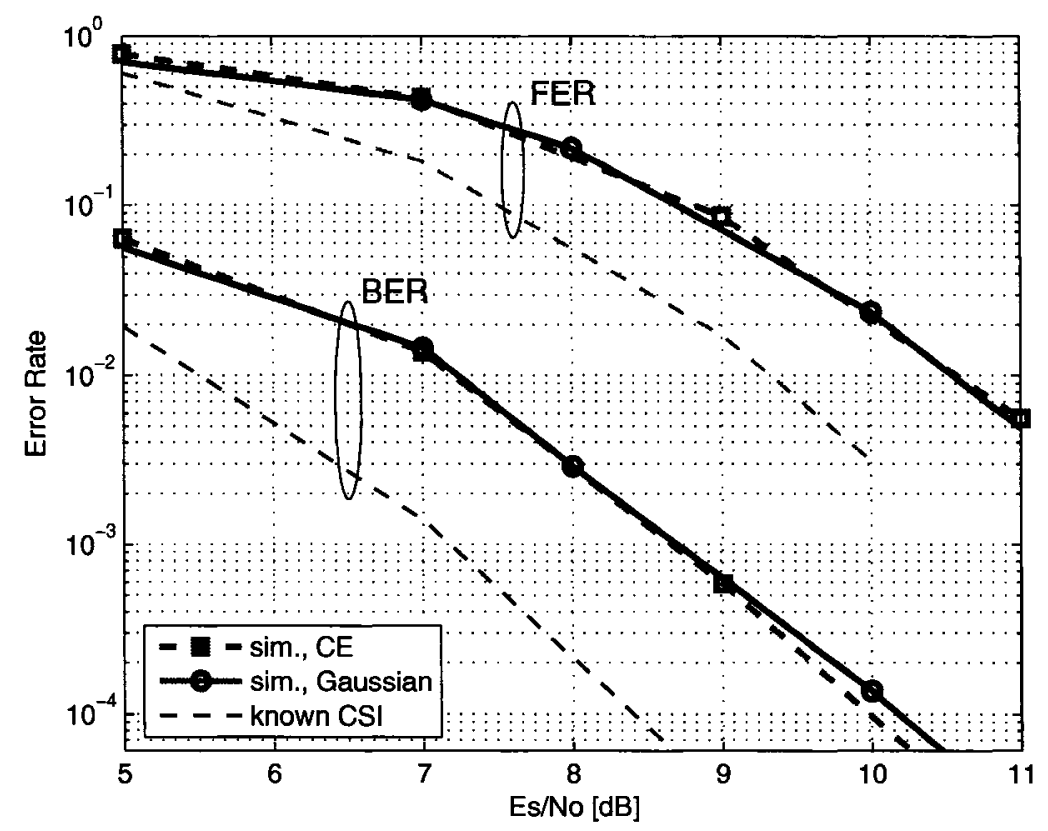

Figure 3.10: Comparison of BER and FER Performance using Different Simulation Approaches for a Coded QPSK System with FET Pilots in WINNER C2 Channel (Parameter Set 2-B) 


\subsection{Summary}

An analytical and a semi-analytical approach for estimating the BER performance of an uncoded QPSK and 16-QAM SC system with CEE have been developed. The effect of CEE on the MSE of the equalizer output was also analyzed. It was found that the MSE of the equalizer output with CEE is the sum of the MSE for the known CSI and the scaled MSE of the channel estimates. By modeling the CEE as a Gaussian random variable, the MSE of the equalizer output for systems with imperfect channel estimates can also be estimated. Using a Gaussian approximation on the residual ISI and equalizer output noise, the MSE of the equalizer output with CEE is then used to obtain the estimated BER.

Using computer simulation, it was shown that both the analytical and semianalytical approaches produce the same estimated MSE of the equalizer output. Moreover, both analytical and semi-analytical approaches can accurately estimate the BER performance of uncoded QPSK and 16-QAM systems. It was also shown that using the semi-analytical approach, the BER and FER performance of a coded QPSK system can be estimated accurately. The approaches developed in this chapter provide alternative tools for estimating the BER performance without simulating the complete system, given the averaged MSE of the CEE and the actual channel frequency response. 


\section{Chapter 4}

\section{Design and Equalization of SC Signals with FDM Pilots}

This chapter provides detailed descriptions of the design of frequency domain multiplexed (FDM) pilots for SC systems and equalization techniques for SC signals with FDM pilots. Two techniques for multiplexing in-band FDM pilots for SC signals are introduced, namely the frequency domain superimposed pilot technique (FDSPT) and the frequency expanding technique (FET). The PAPR effects of multiplexing FDM pilots for SC signals using the GMC transmission technique are addressed, followed by the frequency domain equalization of the SC signals with FDM pilots using a frequency domain LMMSE equalizer and a frequency domain IBSDFE equalizer. Moreover, we propose an iterative decision-directed inter-symbol interference cancellation technique for SC signals with FDSPT pilots. Finally, a brief summary of this chapter is included.

\subsection{Introduction}

In order to fulfill the requirement of flexible and efficient allocation of SC signals to available spectrum, we need to consider the feasibility of utilizing FDM pilots for SC modulated systems, an approach similar to the pilot arrangement of an OFDM system. We consider channel estimation for SC systems with frequency domain equalization using FDM pilot techniques which have been typically used for OFDM systems. 
In particular, we consider the application of two dimensional pilot assisted channel estimation (PACE) techniques, first proposed for OFDM by Hoeher [80], to SC systems. Instead of using the whole OFDM symbol for channel estimation, this PACE technique periodically inserts pilot tones ${ }^{1}$ with equidistant pilot spacing, reducing the pilot overheads $[81,82]$. Aiming for application in time and frequency selective channels, we multiplex multiple pilot tones within the signal bandwidth using an IFDMA signal with a Chu sequence (described in Sec. 2.3.2), which has constant envelope and uniform spectrum. We consider the effects of inserting the pilots in terms of PAPR and compare with that of an OFDM signal. We also compare with the performance for conventional time domain multiplexed (TDM) pilots. With one additional FFT operation and using the generalized multicarrier (GMC) transmission technique [21], the SC signal with pilot tones can be generated.

Two techniques of FDM pilots for SC systems are considered here. One is to superimpose pilot tones onto scaled or deleted data tones, and is termed frequency domain superimposed pilot technique (FDSPT). Early work on the insertion of pilot symbols in the frequency domain for SC systems can be found in [83,84], called transparent tone-inband (TTIB), in which a single reference tone is inserted in the middle of the signal bandwidth using a notch filter along with suitable mixing and filtering in the passband and it is intended for flat fading mobile communication systems. A "data dependent superimposed training" (DDST) technique is introduced in $[85,86]$, and in general form, in [87], to distort the data symbol sequence (in the time domain) to create periodic frequency nulls for insertion of pilot tones. The main difference of our FDSPT technique with parameter $\alpha=0$ (defined later) from those of $[85,86]$ and $[87]$ is that it is defined and implemented in the frequency domain instead of the time domain. It is also applicable to pilots with arbitrary spacing in the frequency domain. Other previous works on superimposed pilots include $[88,89]$ and [90], in which periodic pseudo-random pilots are added to data, and are extracted

\footnotetext{
${ }^{1}$ Pilot tones refer to the pilots in frequency domain, while pilot symbols refer to the pilots in time domain.
} 
(a) FET

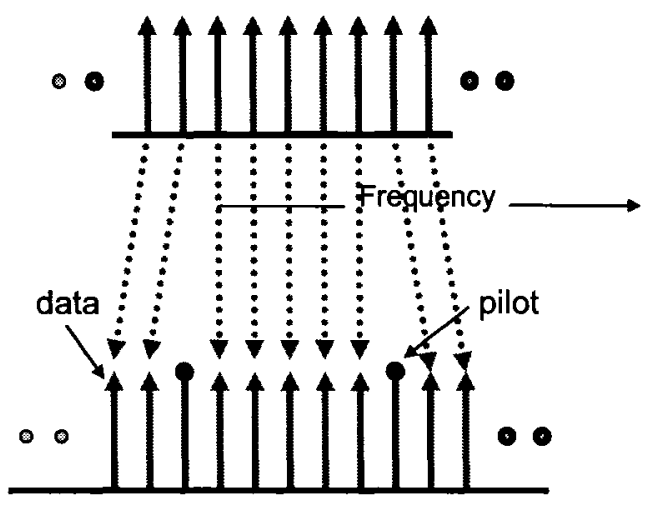

(b) FDSPT

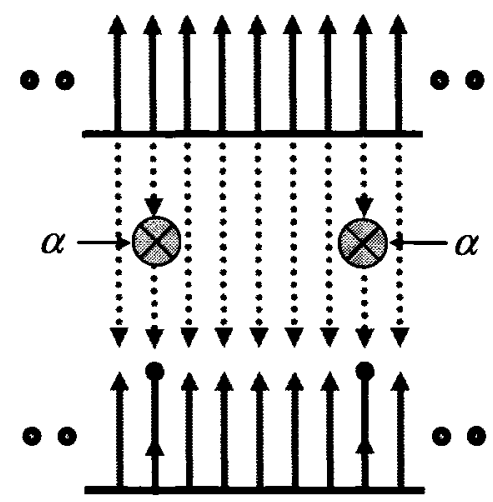

Figure 4.1: Graphical Illustration of Two Variants of FDM Pilots: Frequency Expanding Technique (FET) and Frequency Domain Superimposed Pilot Technique (FDSPT)

for channel estimation at the receiver by time-averaging over multiple periods.

The other main pilot technique considered here is to shift groups of data frequencies for multiplexing of the pilot tones, called frequency expanding technique (FET), a technique usually employed by OFDM systems, where it is called PACE (pilotassisted channel estimation) $[80,81]$. In this thesis, the generation of the transmitted $\mathrm{SC}$ signals with FET pilots is in the frequency domain. Moreover, the general training and precoding framework of [87] includes both FET and FDSPT, but is based on a specific channel estimator (DFT channel estimator). For FET pilots, there is no limitation on the specific channel estimator used.

\subsection{Analysis of SC Signals with FDM Pilots}

\subsubsection{Frequency Domain Superimposed Pilot Technique}

The idea of FDSPT is to periodically scale the data tones for superimposing of the IFDMA pilot tones, as shown in Fig. 4.1 (b). The advantage is not to expand the signal bandwidth, thus maintaining the spectral efficiency. However, as will be shown later, it suffers performance degradation due to the loss of part of the useful data tones and induces slightly higher PAPR than that of SC signals without FDM pilots. We want to obtain an expression for $x_{n}$ in Fig. 2.4, where for FDSPT, $X_{\ell}$ is the $\ell$ th 
element of

$$
\mathbf{X}_{\mathbf{S}}=\left[\beta P_{0}+\alpha A_{0}, A_{1}, \ldots, \beta P_{1}+\alpha A_{K}, A_{K+1}, \ldots, A_{M-1}\right]
$$

where $\alpha$ and $\beta$ are the scaling factors for the data and pilot tones at pilot locations, respectively, $P_{\ell}$ is the $\ell$ th pilot tone and $K$ is the pilot spacing. To analyze the implication of periodically frequency scaling and superimposing in terms of PAPR, define a frequency-scaling window as

$$
Q_{\ell}= \begin{cases}\alpha & \ell \in \Omega \\ 1 & \ell \notin \Omega\end{cases}
$$

where the frequency index of the pilots $\Omega$ is defined in (2.46) and $0 \leq \alpha \leq 1$. The baseband transmitted signal (with IFDMA training signal) $x_{n}$ can then be expressed as

$$
x_{S}(n)=\underbrace{\frac{1}{L} \sum_{\ell=0}^{M-1} A_{\ell} Q_{\ell} e^{j \frac{2 \pi n \ell}{L}}}_{\text {distorted data signal }}+\underbrace{\frac{\beta}{L} \sum_{k=0}^{N-1} P_{k} e^{j \frac{2 \pi n k K}{L}}}_{I F D M A \text { training signal }}
$$

where $0 \leq \beta \leq 1$, the subscript ${ }_{S}$ represents the signal for FDSPT and $L$ denotes the number of oversampled data samples per block. The second term in (4.3) is a deterministic and common term to FDSPT, FET and OFDM signals using an IFDMA signal as a training signal. Therefore, for the purpose of comparing the PAPR performance among the signals using these techniques, we focus on the first term in (4.3), given as

$$
\begin{aligned}
x_{S}^{\prime}(n) & =\frac{1}{L} \sum_{\ell=0}^{M-1} A_{\ell} Q_{\ell} e^{j \frac{2 \pi n \ell}{L}} \\
& =\frac{1}{L} \sum_{\ell=0}^{M-1} A_{\ell} e^{j \frac{2 \pi n \ell}{L}}-\frac{1-\alpha}{L} \sum_{\ell=0}^{M-1} A_{\ell} \sum_{i=0}^{N-1} \delta(\ell-i K) e^{j \frac{2 \pi n \ell}{L}} \\
& =a_{n}^{\prime}-(1-\alpha) \frac{N}{L} \sum_{m=0}^{M-1} a_{m} \frac{\operatorname{sinc}\left(\frac{n}{I}-m\right)}{\operatorname{sinc}\left(K\left(\frac{n}{L}-\frac{m}{M}\right)\right)} e^{j \pi\left(\frac{n}{L}-\frac{m}{M}\right) K(N-1)}
\end{aligned}
$$

where $\left\{a_{n}^{\prime}\right\}_{n=0}^{L-1}$ is the oversampled data samples without frequency scaling. Note that the details of the derivation of (4.4) are shown in Appendix A.3. The second term of (4.4) can be considered as the inter-symbol interference term due to the scaled 

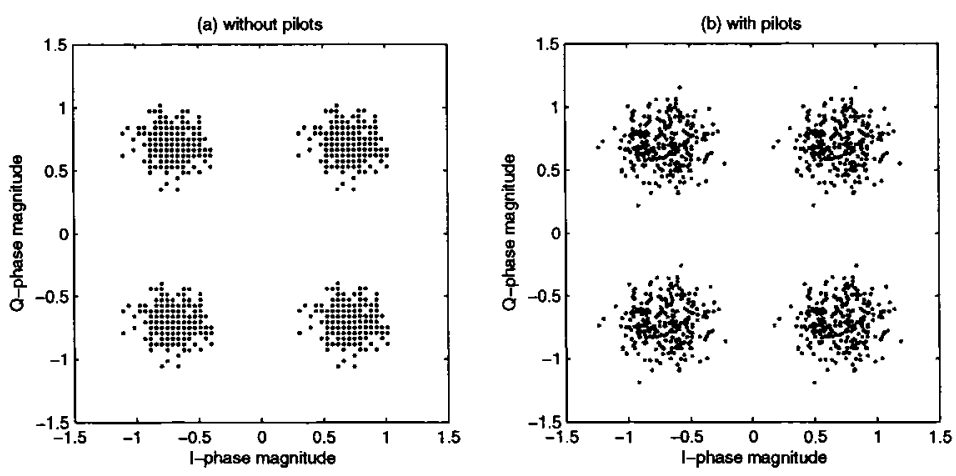

Figure 4.2: Magnitude of I- and Q-Phase of SC Signal with FDSPT with and without IFDMA Training Signal $(\alpha=0, \eta=3.125 \%$, QPSK)

data frequencies. To visualize the effects of the scaling of the data frequency samples, consider the case of a single sample per symbol, i.e. $I=1$ and $L=M$. (4.4) can be simplified as

$$
\begin{aligned}
x_{m}^{\prime \prime} & =a_{m}-(1-\alpha) \frac{N}{M} \sum_{k=0}^{M-1} a_{k} \frac{\operatorname{sinc}(m-k)}{\operatorname{sinc}\left(\frac{m-k}{N}\right)} e^{j \pi\left(\frac{m-k}{M}\right) K(N-1)} \\
& =\left(1-\eta^{\prime}\right) a_{m}-\eta^{\prime} \underbrace{\sum_{\substack{k=0 \\
k \neq m}}^{M-1} a_{k} \frac{\operatorname{sinc}(m-k)}{\operatorname{sinc}\left(\frac{m-k}{N}\right)} e^{j \pi\left(\frac{m-k}{M}\right) K(N-1)}}_{\text {interference term }}
\end{aligned}
$$

where $m=0,1, \ldots, M-1, a_{m}$ is the $m$ th data symbol and $\eta^{\prime}=(1-\alpha) \eta$ and $\eta=\frac{N}{M}=\frac{1}{K}$ is defined as the pilot overhead. It is obvious, from (4.5) that the removal of data frequencies reduces the symbol energy, while adding a random interference term. Fig. 4.2 shows the I-Q plot of the signal without pilots $x_{m}^{\prime \prime}$ and with pilots $x_{S}(n)$, and with data suppressed at the pilot frequencies with $\alpha=0, I=1$ and a pilot overhead of $\eta=3.125 \%$. Although the signal constellation points are still distinguishable, as shown in Fig. 4.2, the PAPR is expected to be slightly higher than that of the SC signals without pilots, as a result of removal of data frequencies for pilot tones.

From (4.4), it is obvious that the envelope fluctuation is the largest when $\alpha=0$. The transmitted signal with $\alpha=0$ and without the pilot tones can be directly derived 
by summing all the data tones, given as

$$
\begin{aligned}
x_{S}^{\prime}(n) & =\frac{1}{L} \sum_{\substack{\ell=0 \\
\ell \neq r K}}^{M-1} X_{\ell} e^{j \frac{2 \pi n \ell}{L}}, \quad r=0,1, \ldots, N-1 \\
& =\frac{1}{L} \sum_{i=0}^{N-1} \sum_{\ell=i K+1}^{(i+1) K-1} A_{\ell} e^{j \frac{2 \pi n \ell}{L}} \\
& =\frac{1}{L} \sum_{i=0}^{N-1} \sum_{\ell=i K+1}^{(i+1) K-1} \sum_{m=0}^{M-1} a_{m} e^{j \frac{2 \pi m \ell}{M}} e^{j \frac{2 \pi n \ell}{L}} \\
& =\frac{1}{L} \sum_{m=0}^{M-1} a_{m} \sum_{i=0}^{N-1} e^{j 2 \pi\left(\frac{n}{L}-\frac{m}{M}\right)(i K+1)} \sum_{\ell=0}^{K-2} e^{j 2 \pi\left(\frac{n}{L}-\frac{m}{M}\right) \ell} \\
& =\frac{M^{\prime}}{L} \sum_{m=0}^{M-1} a_{m} g_{S}\left(n-\frac{m L}{M}\right)
\end{aligned}
$$

where $g_{S}(x)=\frac{\operatorname{sinc}\left(\frac{x}{I}\right)}{\operatorname{sinc}\left(\frac{x}{L}\right)} \frac{\operatorname{sinc}\left(\frac{K-1}{L} x\right)}{\operatorname{sinc}\left(\frac{K}{L} x\right)} e^{\frac{j \pi x}{I}}$ is the sampled impulse response of a channel with periodic nulls, $M^{\prime}=M-N$ and $n=0,1, \ldots, L-1$. The high sidelobes of the $g_{S}\left(n-\frac{m L}{M}\right)$ pulse increase the PAPR.

\subsubsection{Frequency Expanding Technique (FET)}

The FET evenly expands groups of frequencies for multiplexing of IFDMA pilot tones, as shown in Fig. 4.1 (a). The FET pilot scheme has slightly lower spectral efficiency due to the expansion of data frequencies to accommodate for the pilot tones, which results in no performance loss but slightly higher PAPR than that of the conventional SC signal. Note that the FET is the frequency domain pilot technique commonly used in OFDM systems. When using FET, the multiplexed data and pilot tones $X_{\ell}$ is the $\ell$ th element of

$$
\mathbf{X}_{\mathbf{E}}=\left[P_{0}, A_{0}, \ldots, P_{1}, A_{K-1}, \ldots, P_{N-1}, \ldots, A_{M-N-1}\right]
$$

where pilot tones $P_{\ell}$ are periodically multiplexed with the data tones $A_{\ell}$. Note that $N$ more data symbols can be sent using FDSPT. The baseband transmitted samples 
$x_{n}$ in Fig. 2.4 is given as

$$
x_{E}(n)=\underbrace{\frac{1}{L} \sum_{\substack{\ell=0 \\ \ell \neq i K}}^{M-1} X_{\ell} e^{j \frac{2 \pi n \ell}{L}}}_{\text {distorted data signal }}+\underbrace{\frac{1}{L} \sum_{k=0}^{N-1} P_{k} e^{j \frac{2 \pi n k K}{L}}}_{\text {IFDMA training signal }}
$$

where the subscript $_{E}$ represents the signal for FET. Similar to the FDSPT case, we consider the distorted data signal term in (4.8), which can be further expanded as

$$
\begin{aligned}
x_{E}^{\prime}(n) & =\frac{1}{L} \sum_{\substack{\ell=0 \\
\ell \neq r K}}^{M-1} X_{\ell} e^{j \frac{2 \pi n \ell}{L}}, \quad r=0,1, \ldots, N-1 \\
& =\frac{1}{L} \sum_{i=0}^{N-1} \sum_{\ell=i K+1}^{(i+1) K-1} A_{\ell-i-1} e^{j \frac{2 \pi n \ell}{L}} \\
& =\frac{1}{L} \sum_{i=0}^{N-1} \sum_{\ell=i K+1}^{(i+1) K-1} \sum_{m=0}^{M^{\prime}-1} a_{m} e^{-j \frac{2 \pi m(\ell-i-1)}{M^{\prime}}} e^{j \frac{2 \pi n \ell}{L}} \\
& =\frac{M^{\prime}}{L} e^{j \frac{\pi n}{I}} \sum_{m=0}^{M^{\prime}-1} a_{m} g_{E}(m, n) e^{-j \pi\left(\frac{\left(M^{\prime}-1\right) m}{M^{\prime}}\right)}
\end{aligned}
$$

where $g_{E}(m, n)=\frac{\operatorname{sinc}\left(N\left(K n / L-m(K-1) / M^{\prime}\right)\right)}{\operatorname{sinc}\left(K n / L-m(K-1) / M^{\prime}\right)} \times \frac{\operatorname{sinc}\left((K-1)\left(n / L-m / M^{\prime}\right)\right)}{\operatorname{sinc}\left(n / L-m / M^{\prime}\right)}, M^{\prime}=M-N$ and $n=0,1, \ldots, L-1$. It is obvious that the time-varying modified pulse shaping filter $g_{E}(m, n)$ results in higher PAPR than a SC system without multiplexing pilot tones, as further shown in Sec. 4.2.3. Fig. 4.3 shows the I-Q plot of the transmitted waveform without pilots $s_{F E T}^{\prime}(n)$ and with pilots $s_{F E T}(n)$ with $I=1$ and $3.125 \%$ pilot overhead. In contrast to Fig. 4.2 (a), the signal constellation points are not distinguishable, due to the expanding of the signal bandwidth. Note that the signal constellation points can be recovered after removing the pilot tones and shifting the groups of the data tones to their original locations, subjected to noise power. The PAPR for the signal with and without pilots is similar, as shown in Fig. 4.2(b). It is interesting to see that by comparing Fig. 4.3 with Fig. 4.2 the SC signal with FET pilots has slightly higher PAPR than that of the SC signal with FDSPT pilots. 

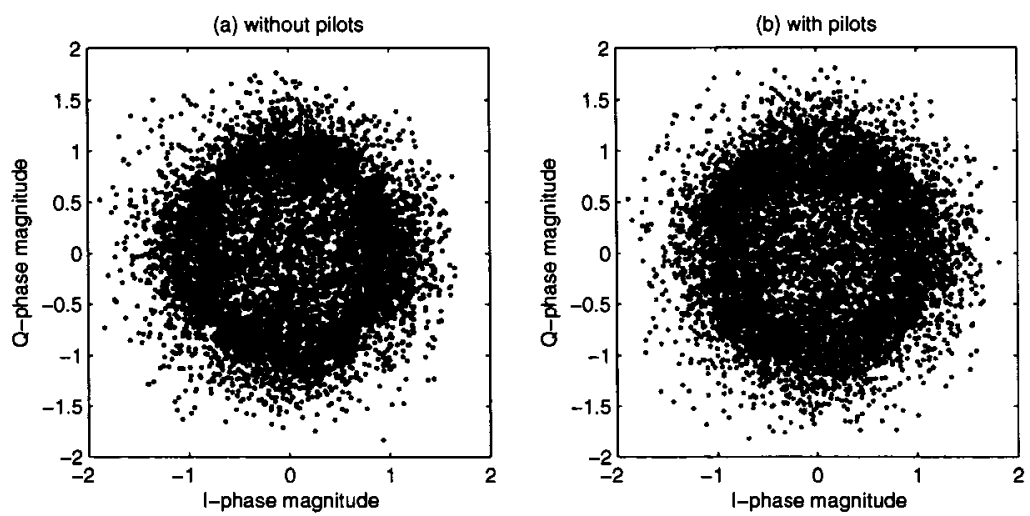

Figure 4.3: Magnitude of I- and Q-Phase of SC Signal for FET with and without IFDMA Pilot Signal $(\eta=3.125 \%$, QPSK)

\subsubsection{PAPR and Power Backoff Considerations}

For an OFDM system, the probability that a PAPR value exceeds a certain value depends on the number of subcarriers. The larger the number of the subcarriers, the higher the probability [93]. We want to compare the number of data symbols contributing to the higher PAPR for (4.6) and (4.9) with that of an OFDM system. First consider the transmitted samples for OFDM, generated using Fig. 2.4 without the FFT prior to the MUX operation, given as

$$
x_{O}^{\prime}(n)=\frac{1}{L} \sum_{\substack{m=0 \\ m \neq i K}}^{M-1} a_{m} e^{j \frac{2 \pi m n}{L}}, \quad n=0,1, \ldots, L-1
$$

where the subscript $o$ represents the signal for OFDM. From (4.10), there are $(M-N)$ random data symbols contributing to the PAPR. Note that $N=M / K$. For SC modulated with FDSPT with $\alpha=0$ and FET, the number of random data symbols contributing significantly to the PAPR is much less than $(M-N)$ due to the modified sinc pulse shaping filters in both cases. Hence, the PAPR for a SC system with FDSPT or FET is lower than that of an OFDM system with the same amount of pilot tones. It is also shown that SC modulated signal with FET has slightly higher PAPR than that of FDSPT.

We use simulation to further verify our analysis on the effects of adding FDM 


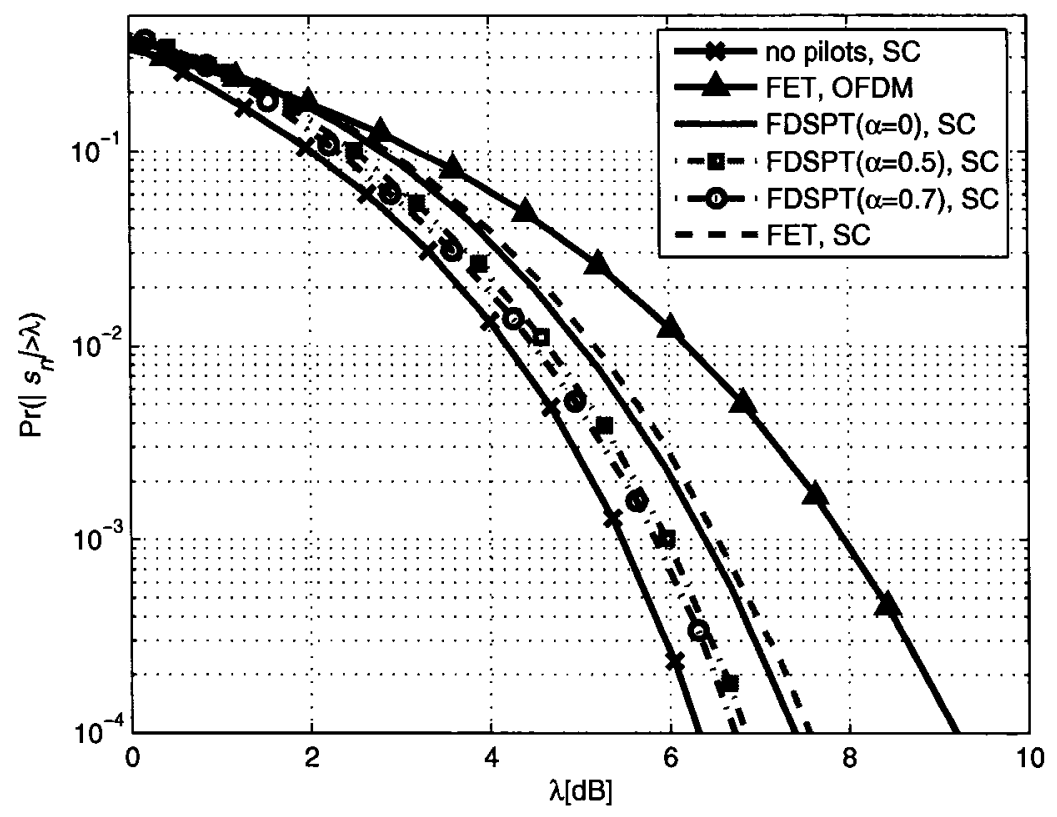

Figure 4.4: Comparison of CCDF of Magnitude of $x(n)$ for SC Modulated Signals with FDM Pilots (Parameter Set 1)

pilots. Consider Fig. 4.4, where $x_{n}$ is either from (4.3) or (4.8). Parameter Set 1 $(M=826, L=826 \times 12, N=118)$ is used for the simulation. The FDSPT with $\alpha=0$ and FET have about $2 \mathrm{~dB}$ advantage over OFDM and $1.5 \mathrm{~dB}$ disadvantage over SC modulated signal without pilot tones at complementary cumulative distribution function $(\mathrm{CCDF})=10^{-4}$. The higher the value of $\alpha$ is, the lower the PAPR. However, the higher the value of $\alpha$ the worse the performance of the channel estimator. There exists a fundamental trade-off between the PAPR and the performance of the channel estimator.

Fig. 4.5 shows the spectrum generated using the SC modulated signal with FDSPT with various $\alpha$ values, FET and OFDM with IFDMA pilot signal with a Chu sequence, where $\rho$ is the power backoff of a high power amplifier (HPA) in dB. Note that parameter Set $1(M=826, L=826 \times 12, N=118)$ is used for the simulation. The non-linear high power amplifier (HPA) model used is the Rapp model [113] with $p_{o}=2$, where $p_{o}$ is defined in Sec. 7.5 when we discuss the issue of reducing PAPR 


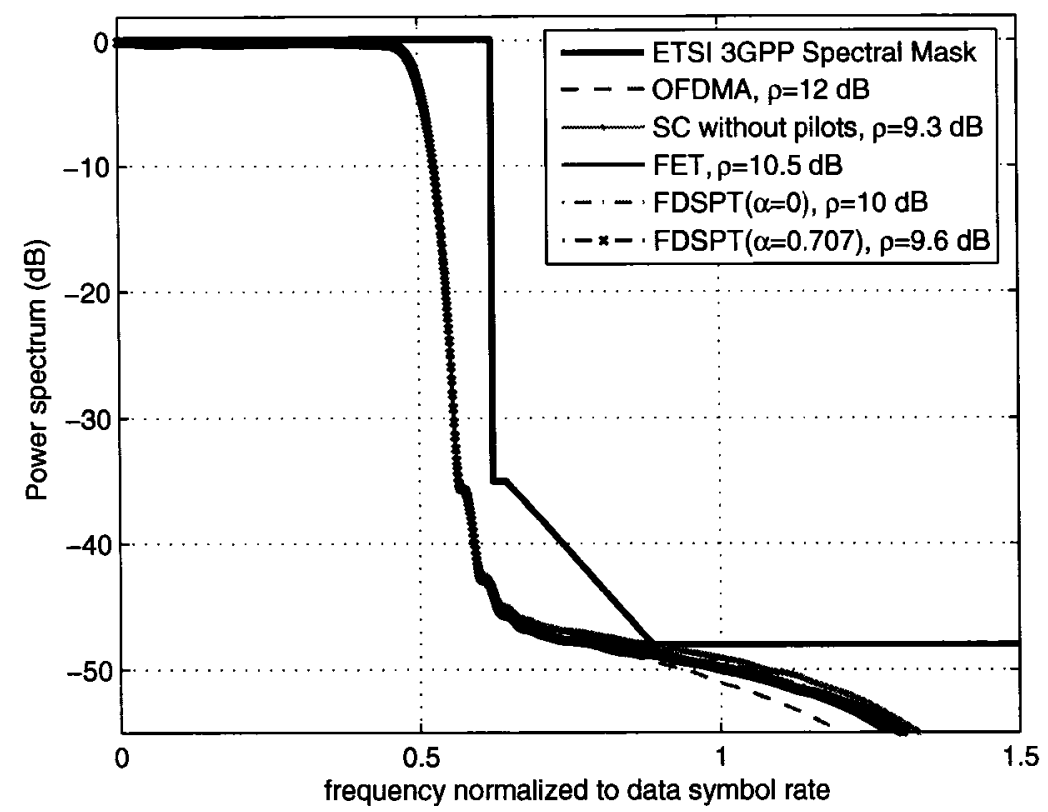

Figure 4.5: Power Spectra of Signal Emerging from a Rapp Model $\left(p_{o}=2\right)$ Power Amplifier, with Backoffs Required to Satisfy a Spectral Mask (Parameter Set 1)

of SC signals with FDM pilots. The spectrum for the system without FDM pilots is the same as that for the TDM pilots. The ETSI 3GPP spectral mask is scaled to fit the generated spectrum. The backoff values, used for illustrative purposes, are obtained by trial and error such that the power spectrum is just within the mask. As expected, the OFDM signal requires the largest backoff, while the SC without pilots requires the least. The $\mathrm{SC}$ with $\mathrm{FET}$ requires $0.5 \mathrm{~dB}$ more backoff than that of $\mathrm{SC}$ with FDSPT $(\alpha=0)$. Note that the spectral mask could have been shifted slightly to the right for FDSPT, since its net data symbol rate is slightly higher, although this has not been done in Fig. 4.5.

\subsection{Channel Equalization of SC Signals with FDM Pilots}

Channel equalization of SC signals with FDM pilots is performed in the frequency domain. The FDM pilots are first removed and then equalized using either frequency 
domain LMMSE or frequency domain IBSDFE, applying the corresponding channel frequency response at the data frequencies.

\subsubsection{Frequency Domain LMMSE Equalization}

First consider FDSPT. Given that the channel is known, the pilot tones are removed from the received signal tones before equalization,

$$
\begin{aligned}
Y_{\ell}^{\prime} & =Y_{\ell}-\beta H_{\ell} P_{\ell / K} \\
& =\alpha H_{\ell} A_{\ell}+V_{\ell}, \quad \ell \in \boldsymbol{\Omega}
\end{aligned}
$$

where the set pilot frequency indices $\Omega$ is defined in (2.46). At $\ell \notin \Omega$, from (2.8), the received data frequencies are given as $Y_{\ell}=H_{\ell} A_{\ell}+V_{\ell}$. The linear MMSE equalizer taps for a single received sample with FDSPT can then be calculated as

$$
C_{\ell}=\frac{H_{\ell}^{\prime *}}{\left|H_{\ell}^{\prime}\right|^{2}+\sigma^{2}}, \quad \ell=0,1, \ldots, M-1
$$

where ${ }^{*}$ denotes the complex conjugate and

$$
H_{\ell}^{\prime}= \begin{cases}\alpha H_{\ell} & \ell \in \Omega \\ H_{\ell} & \ell \notin \Omega .\end{cases}
$$

The corresponding MMSE of the linear equalizer can be shown to be

$$
J_{S}=\frac{\sigma^{2}}{M} \sum_{\ell=0}^{M-1} \frac{1}{\left|H_{\ell}^{\prime}\right|^{2}+\sigma^{2}}
$$

The estimated data symbols using FDSPT can be obtained by taking the $M$-point inverse FFT (IFFT) of $\left\{C_{\ell} Y_{\ell}^{\prime \prime}\right\}_{\ell=0}^{M-1}$, where

$$
Y_{\ell}^{\prime \prime}= \begin{cases}Y_{\ell}^{\prime}, & \ell \in \boldsymbol{\Omega} \\ Y_{\ell}, & \ell \notin \Omega .\end{cases}
$$

Using (2.26), the equalization of SC signal with FET is performed as $\tilde{A}_{\ell}=Y_{\ell} C_{\ell}$, where $\ell=0,1, \ldots, M-1$ and $\ell \notin \Omega$. The MMSE of the linear equalizer for FET can be shown to be

$$
J_{E}=\frac{\sigma^{2}}{M-N} \sum_{\substack{\ell=0 \\ \ell \notin \Omega}}^{M-1} \frac{1}{\left|H_{\ell}\right|^{2}+\sigma^{2}}
$$


Let $\tilde{\mathbf{A}}=\left[\tilde{A}_{1}, \ldots, \tilde{A}_{K-1}, \tilde{A}_{K+1}, \ldots, \tilde{A}_{M-1}\right]$. The estimated data symbols using FET can be obtained by taking the $(M-N)$-point IFFT of $\tilde{\mathbf{A}}$. The BER for both cases (assuming known channel) can be estimated as

$$
B E R \approx \mathrm{Q}\left(\sqrt{\frac{1-J}{J}}\right)
$$

where $J$ is either $J_{S}$ or $J_{E}$ and where the residual error is assumed to be Gaussian (see Chapter 3). Since the channel is assumed to be known in the derivation of (4.17), (4.17) can be considered as a lower bound on the BER that can be achieved with FDSPT and FET pilots.

Fig. 4.6 depicts the BER for an uncoded system using FDSPT with different values of $\alpha$ using frequency domain LMMSE, given the channel is known. Parameter Set 1 (see Table 2.1 and Table 2.2) is used in the simulation for both the uncoded and coded cases. Note that $\frac{E_{b}}{N_{0}}$ in Fig. 4.6 is calculated according to $(2.23)$ with $N_{p}=0$ for FDSPT and $N_{p}=N$ for FET. We assume the channel is static for the uncoded case. The BER performance of FET and no pilots are also included for comparison. The theoretical results are consistent with the simulation results. The larger the value of $\alpha$ or the smaller the value of $\eta$ (pilot overhead defined as $1 / K$ ) is, the better the BER. The performance degradation for FDSPT with $\alpha=0$ is significant due to the large value of $\eta$ and the presence of pilots in every block. Channel coding can be used to compensate for part of the degradation (see Fig. 4.11).

\subsubsection{Frequency Domain IBSDFE Equalization}

First consider FDSPT. The feedback and feedforward frequency domain IBSDFE equalizer taps described in Section 2.4 .2 at the $i$ th iteration, denoted as $B_{\ell}^{(i)}$ and $C_{\ell}^{\prime(i)}$, for a single received frequency sample can be calculated using (2.36) and (2.37)

with $H_{\ell}$ being replaced by $H_{\ell}^{\prime}$, defined in (4.13). The corresponding MMSE of the frequency domain IBSDFE at the $i$ th iteration can then be calculated using (2.40) with $H_{\ell}$ being replaced by $H_{\ell}^{\prime}$. The estimated data symbols using FDSPT can be 


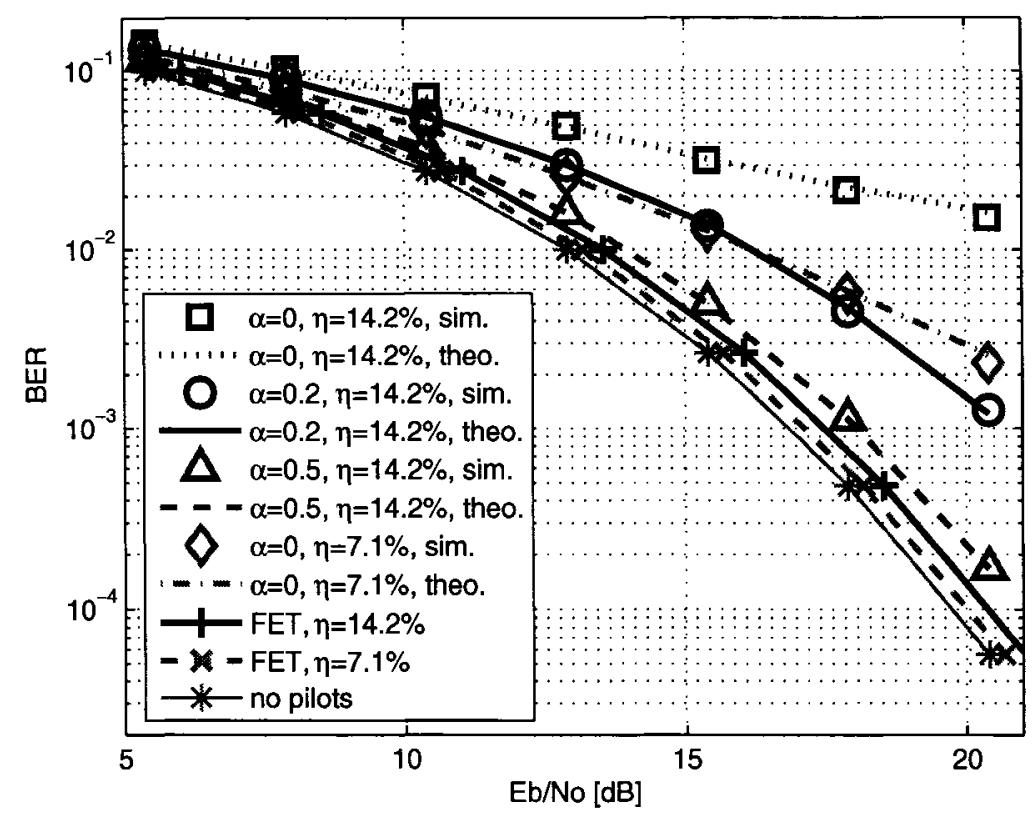

Figure 4.6: BER Performance of FDSPT and FET for Uncoded System using frequency domain LMMSE Equalizer with Known CIR (Parameter Set 1)

obtained by taking the $M$-point IFFT of $\mathbf{U}^{\prime(i)}$, which is given as

$$
\mathbf{U}^{(i)}=\mathbf{Y}^{\prime \prime} \mathbf{C}^{(i) *}+\mathbf{B}^{\prime *} \overline{\mathbf{A}}^{(i-1)}
$$

where $Y_{\ell}^{\prime \prime}$ is defined in (4.15). The equalization of SC signal with FET is performed using (2.28) with $\ell \notin \Omega$. The estimated data symbols using FET can be obtained by taking the $(M-N)$-point IFFT of $\mathrm{U}^{(i)}$ given in (2.28) with $\ell \notin \Omega$. The corresponding MMSE of the frequency domain IBSDFE at the $i$ th iteration for FET can then be calculated using (2.40) with $\ell \notin \Omega$.

Since the residual error of the frequency domain IBSDFE cannot be well characterized as Gaussian, (4.17) cannot be used to estimate the corresponding BER. We use computer simulation to obtain the BER for systems with FDSPT pilots using frequency domain IBSDFE, with known CSI. Fig. 4.7 shows the BER for an uncoded QPSK system with FDSPT pilots with different values of $\alpha$ using frequency domain IBSDFE, given the channel is known. Parameter Set 1 (see Table 2.1 and Table 2.2) are used in the simulation for both the uncoded and coded cases. Note 
that $\frac{E_{b}}{N_{0}}$ in Fig. 4.7 is calculated according to (2.23) with $N_{p}=0$ for FDSPT and $N_{p}=N$ for FET. The BER performance of FET and no pilots are also included for comparison. For $\alpha=0$ and the pilot overhead ratio $\eta=14.2 \%$, error floor occurs at $\mathrm{BER}=10^{-2}$, resulting from a large ratio of the removal of data tones for pilots. For $\alpha=0.2$ and $\eta=14.2 \%$, the performance degradation is about $1.2 \mathrm{~dB}$, relative to the FET pilots with the same pilot overhead. When the pilot overhead is reduced by half, i.e. $\eta=7.1 \%$, FET and FDSPT with $\alpha=0$ produce similar BER performance. In general, using frequency domain IBSDFE provides better interference (due to the scaling of data tones at the pilot frequencies) cancellation capability than that of using linear MMSE equalizer, as shown in Fig. 4.7. Again, since the channel is assumed to be known in obtaining the BER for frequency domain IBSDFE, the BER can be considered as a lower bound on the BER that can be achieved with FDSPT and FET pilots.

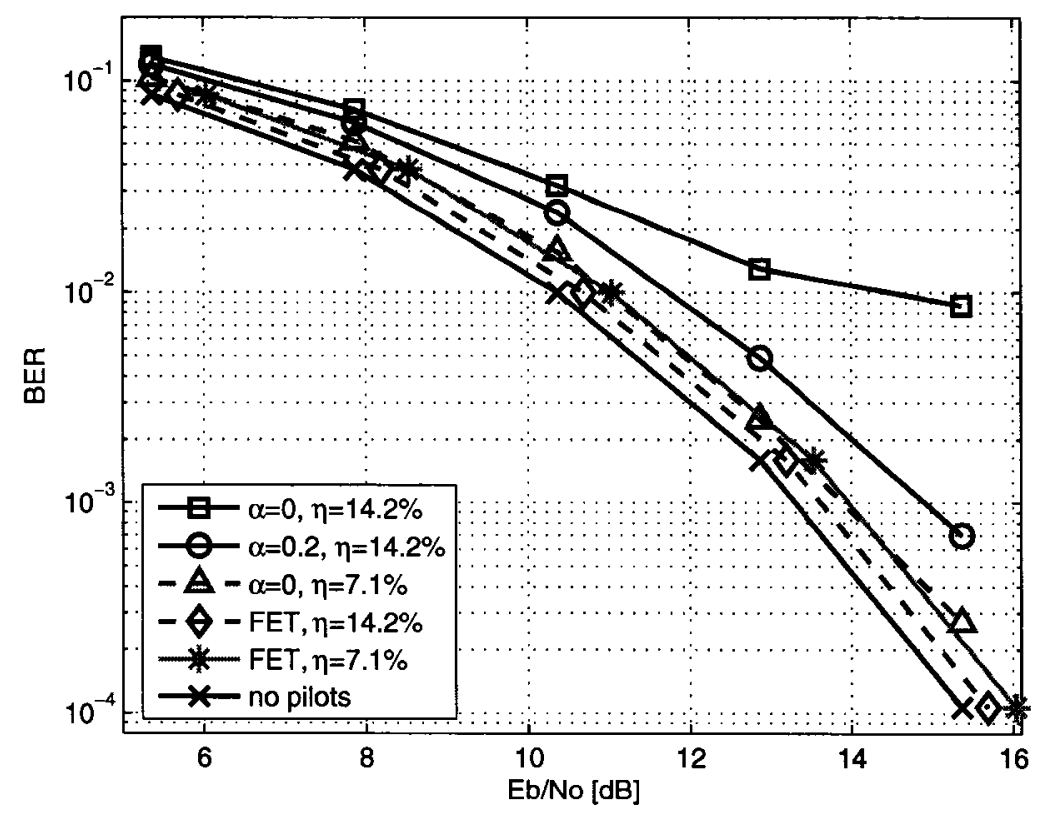

Figure 4.7: BER Performance of FDSPT and FET for Uncoded System using frequency domain IBSDFE Equalizer with Known CIR (Parameter Set 1) 


\subsection{Interference Cancellation of SC Signals with FDSPT pilots}

The removal of the data tones for superimposing of the pilot tones results in BER performance degradation. The second term of (4.4) can be considered as the intersymbol interference term due to the scaled data tones. It is obvious that the interference level decreases with $\alpha$ and increases with $\eta$. Given the estimated channel frequency response (CFR), denoted as $\tilde{H}_{\ell}$, and the DFT of the data decisions $\left\{\hat{a}_{m}\right\}$ either from the frequency domain equalizer (FDE) or the decoder output, denoted as $\hat{A}_{\ell}$, we can compensate for the removal (or scaling) of the data tones by re-constructing the received frequency samples using hard decisions at the pilot frequencies $\Omega$, as shown in Fig. 4.8. After the initial iteration, Switch $S^{\prime}$ is at the left hand side for $\ell \notin \Omega$ and at

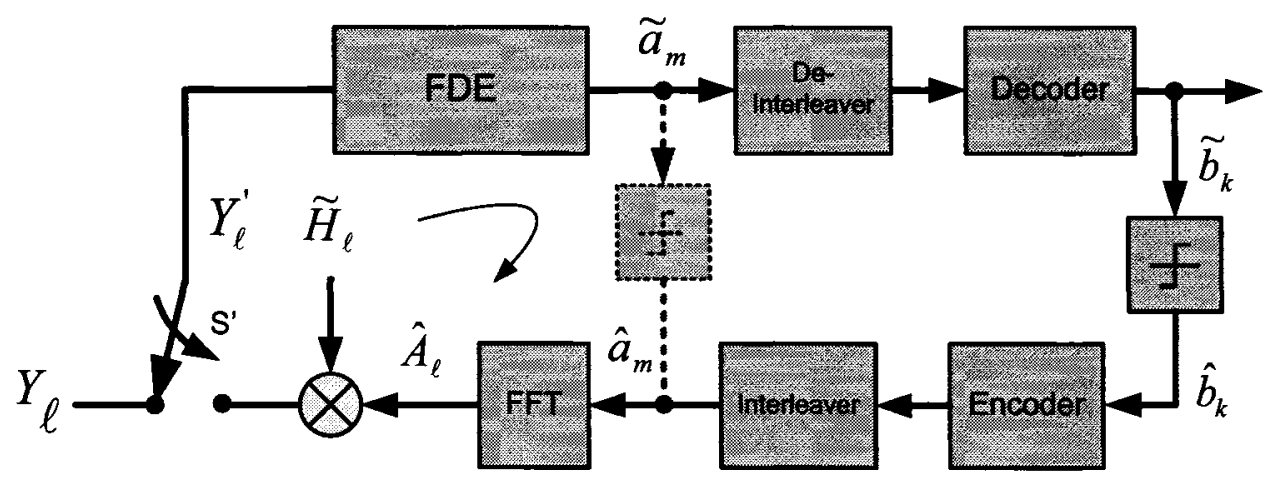

Figure 4.8: Block Diagram of the Proposed Decision-directed Interference Cancellation Technique for SC Signals with FDSPT Pilots

the right hand side for $\ell \in \Omega$. The compensated received frequency samples, denoted as $Y_{\ell}^{\prime}$, can be obtained as

$$
Y_{\ell}^{\prime}= \begin{cases}\tilde{H}_{\ell} \hat{A}_{\ell} & \ell \in \Omega \\ H_{\ell} A_{\ell}+V_{\ell} & \ell \notin \Omega\end{cases}
$$

where $V_{\ell}$ is the $\ell$ th noise frequency sample. Notice that the difference between $Y_{\ell}^{\prime}$ and $Y_{\ell}$ (see (2.8)) is that for $\ell \in \Omega, Y_{\ell}$ contains the received frequency samples for the transmitted pilot tones, while $Y_{\ell}^{\prime}$ contains the received frequency samples for the 
transmitted data tones. Moreover, to obtain $Y_{\ell}^{\prime}$, the DFT of the data decisions and CFR are needed. Note that the $\ell$ th received frequency sample for a block without pilots, denoted as $\bar{Y}_{\ell}$, is given as

$$
\bar{Y}_{\ell}=H_{\ell} A_{\ell}+V_{\ell}, \quad \ell=0,1, \ldots, M-1
$$

where $M$ is the number of data symbols per block.

The objective of the interference cancellation algorithm for SC signal with FDSPT pilots is to obtain $Y_{\ell}^{\prime}$, using $\hat{A}_{\ell}$ and $\tilde{H}_{\ell}$, such that $Y_{\ell}^{\prime} \approx \bar{Y}_{\ell}$ for $\ell=0,1, \ldots, M-1$. Let $\tilde{H}_{\ell}=H_{\ell}+\Delta H_{\ell}$ and $\hat{A}_{\ell}=A_{\ell}+\Delta A_{\ell}$, where $\Delta H_{\ell}$ and $\Delta A_{\ell}$ are defined as the channel estimation and the decision errors at the $\ell$ th frequency, respectively. $Y_{\ell}^{\prime}$, can be re-written as

$$
Y_{\ell}^{\prime}= \begin{cases}H_{\ell} A_{\ell}+V_{\ell}^{\prime} & \ell \in \Omega \\ H_{\ell} A_{\ell}+V_{\ell} & \ell \notin \Omega\end{cases}
$$

where $V_{\ell}^{\prime}$ is the interference due to the channel estimation and data decision errors, given as

$$
V_{\ell}^{\prime}=\Delta H_{\ell} \Delta A_{\ell}+\Delta H_{\ell} A_{\ell}+H_{\ell} \Delta A_{\ell}
$$

Comparing (4.21) with (4.20), we notice that the difference between $Y_{\ell}^{\prime}$ and $\bar{Y}_{\ell}$ is the noise term at the pilot frequencies $\Omega$. Consider the variance of $V_{\ell}^{\prime}$, which is given as

$$
\sigma_{V_{\ell}^{\prime}}^{2} \triangleq \sigma_{\Delta H_{\ell}}^{2} \sigma_{\Delta A_{\ell}}^{2}+\sigma_{\Delta H_{\ell}}^{2}+\sigma_{\Delta A_{\ell}}^{2}
$$

where we assume that $\Delta H_{\ell}, A_{\ell}, H_{\ell}$ and $\Delta A_{\ell}$ are jointly uncorrelated and the variance of $A_{\ell}$ and $H_{\ell}$ are normalized to 1. $\sigma_{\Delta H_{\ell}}^{2}$ and $\sigma_{\Delta A_{\ell}}^{2}$ are the variance of channel estimation and data decision errors at the $\ell$ th frequency. Note that in the absence of channel estimation and data decision errors, i.e. $\sigma_{V_{\ell}^{\prime}}^{2}=0$, the received frequency samples at $\Omega$ are noiseless. In this case, the performance of the FDE would be at least as good as that without FDSPT pilots.

Fig. 4.9 shows the BER performance of an uncoded QPSK system using FDSPT pilots $(\alpha=0)$ in the WINNER C2 channel (see Table 2.3) with iterative decisiondirected interference cancellation. Parameter Set 2-B is used for the simulation. The 
CSI is assumed to be known, i.e. $\sigma_{\Delta H_{\ell}}^{2}=0$. Note that $\sigma_{\Delta A_{\ell}}^{2} \neq 0$. A linear MMSE equalizer is used to show the performance of the iterative interference cancellation technique. For $\eta=6.25 \%$, the BER performance is about $0.8 \mathrm{~dB}$ away from the one without FDSPT pilots. The irreducible BER degradation, resulting from the data decision errors, is more obvious for larger values of $\eta$, such as $\eta=12.5 \%$. Moreover, one iteration gives similar performance to that of three iterations.

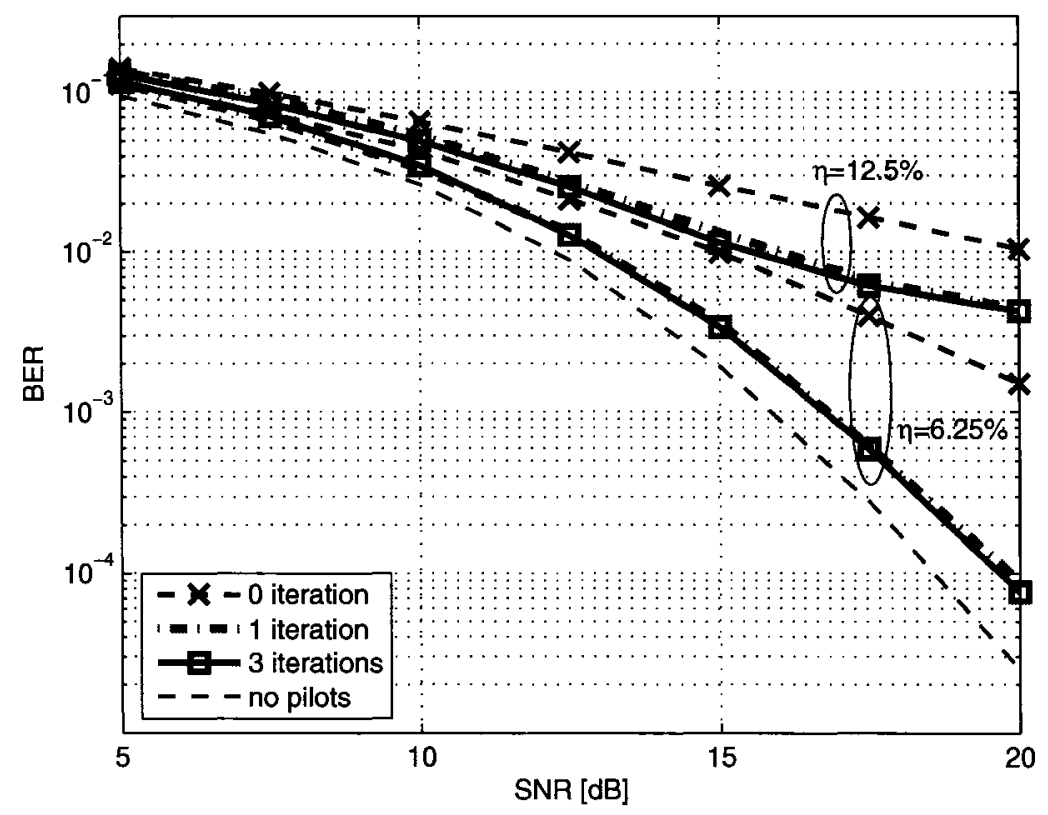

Figure 4.9: BER Performance of FDSPT for Uncoded System using Linear MMSE Equalizer with Iterative Decision-directed Interference Cancellation and known CSI (Parameter Set 2-B)

The performance improvement is not obvious when an IBSDFE (instead of linear MMSE) equalizer is used, resulting from the existing interference cancellation capability of the feedback filter within the IBSDFE equalizer. Consider the equalization of the received frequency samples at $\Omega$ for SC signals with FDSPT signals $(\alpha=0)$ without the proposed interference canceler. Assuming channel frequency nulls at $\boldsymbol{\Omega}$, i.e. $H_{\ell}=0$ for $\ell \in \Omega$ and using (4.18), it can be shown that

$$
U_{\ell}^{(i)}=\bar{A}_{\ell}^{(i-1)}, \quad \ell \in \Omega
$$


where $\bar{A}_{\ell}^{(i-1)}$ is the $\ell$ th frequency of the DFT of the feedback soft decisions from the $(i-1)$ th iteration. When a linear equalizer is used, we have

$$
U_{\ell}^{(i)}=0, \quad \ell \in \Omega
$$

Comparing (4.24) and (4.25), we see that the IBSDFE equalizer provides essentially the same degree of interference cancellation capability for SC signals with FDSPT pilots. With the proposed interference canceler, we have

$$
U_{\ell}^{(i)}=C_{\ell}^{(i)} Y_{\ell}^{\prime}+B_{\ell}^{(i) *} \bar{A}_{\ell}^{(i-1)}, \quad \ell \in \Omega
$$

where $B_{\ell}^{(i)}$ and $C_{\ell}^{(i)}$ are defined in (2.36) and (2.37) respectively. Comparing (4.24) and (4.26), we see the difference is that feedforward and feedback filter are applied on the received frequency samples $Y_{\ell}^{\prime}$ and $\bar{A}_{\ell}^{(i-1)}$ when the proposed canceler is used. Note that this difference is applicable only at the pilot frequencies $\boldsymbol{\Omega}$. Hence, we can conclude that the IBSDFE equalizer with decision directed interference cancellation technique at $\boldsymbol{\Omega}$ is almost equivalent to a version of IBSDFE in which the forward filter is after the feedback and subtraction operation. The only differences are (1) in IBSDFE with interference cancellation, hard decisions are subtracted, instead of soft decisions; and (2) in IBSDFE, $Y_{\ell}$ is forced to zero for pilot tones, since there is no data there (or $\alpha=0$ ). Their BER performance for uncoded QPSK system are similar, as shown in Fig. 4.10.

\subsection{Simulation Results}

We evaluate the BER performance for a coded SC system with non-iterative channel estimator FFT-LLSLF (Linear Least Square Line Fitting) (to be described in Sec. 5.2.1) using FDSPT and FET pilots. Our goal is to compare the performance of the channel estimation using FET and FDSPT pilots with different values of $\alpha$. Parameter Set 1 is used for the simulation (see Table 2.1 and Table 2.2). Note that the BER and FER curves are obtained from 10000 frames if the number of bits 


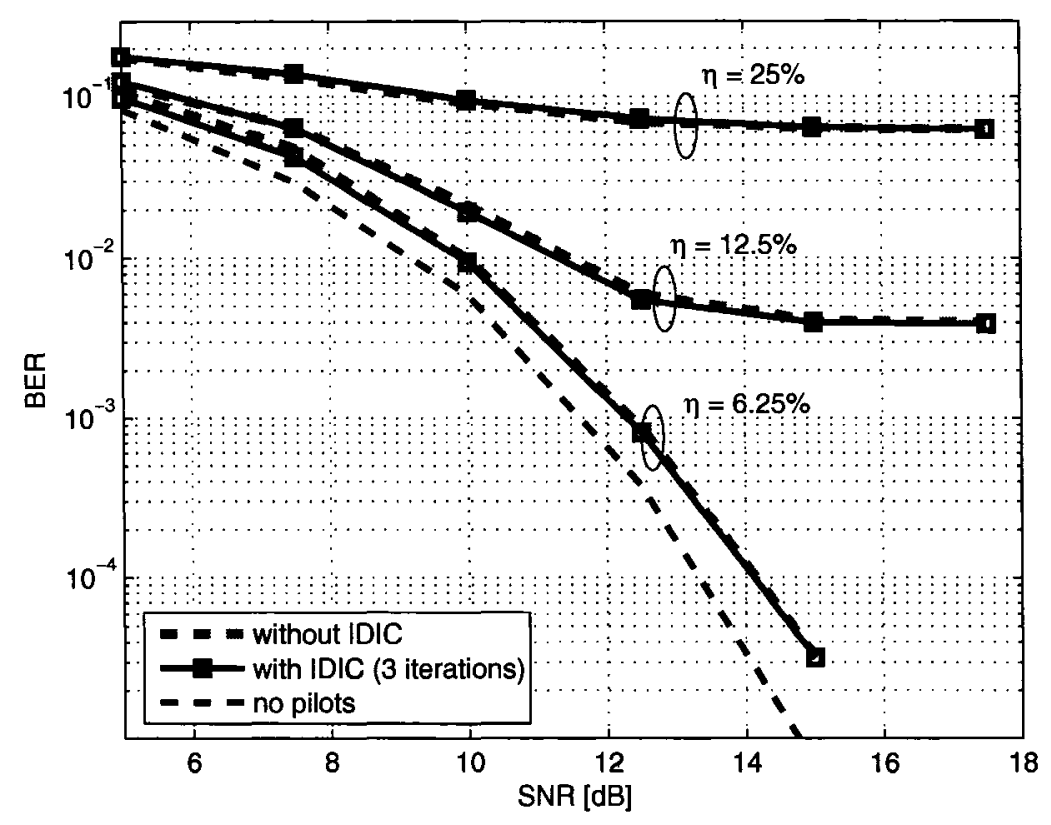

Figure 4.10: BER Performance of FDSPT $(\alpha=0)$ for Uncoded System using IBSDFE Equalizer with Known CSI (IDIC: iterative decision-directed interference cancellation), $\mathrm{Pa}$ rameter Set 2-B

in errors is less than 100000. BER with channel estimation using TDM pilots is also included for comparison. The pilots are added in every frame of 10 blocks according to the row vector [1100110011], where an ' 1 ' represents pilots being added and a ' 0 ' represents no pilots being added in the corresponding blocks. Fig. 4.11 shows the BER for the system with channel estimation using TDM and FDM pilots, where $N=118$ pilots are used for blocks with pilots and $\frac{E_{b}}{N_{0}}$ is defined in (2.23). Note that frequency domain LMMSE equalizer is used. We observe that with the same number of pilots for a frame, TDM and FDM pilot arrangements have similar BER performance. Note that using the TDM pilots, the spectral efficiency is slightly lower than that of using the FDM pilots. In the time domain, an extra $\mathrm{CP}$ of length- $L_{c p}$ is required so that circular convolution can be used for both the training and data symbol block. On the other hand, the two dimensional FDM pilot arrangement requires that a maximum pilot spacing in frequency and time axis is satisfied, which depends on the maximum 


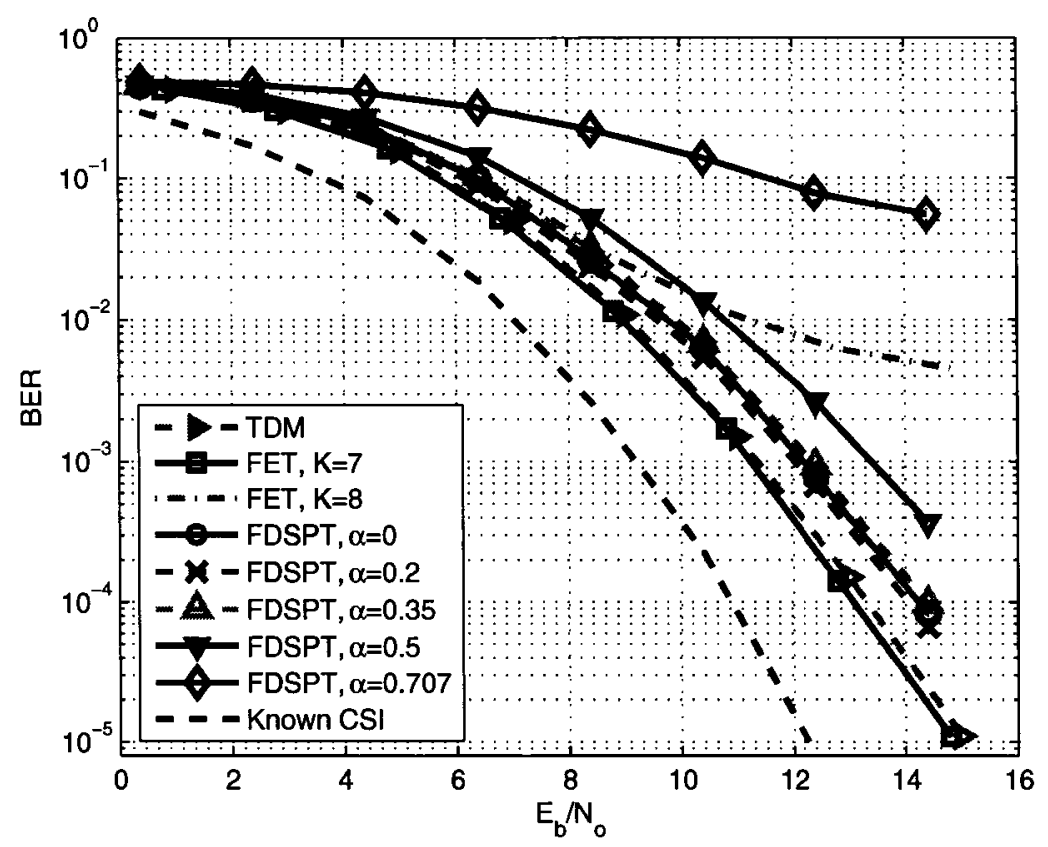

Figure 4.11: BER Performance of FDM Pilots for Coded System using frequency domain LMMSE Equalizer with Channel Estimation (Parameter Set 1)

delay of the channel and the maximum Doppler frequency, respectively. Exceeding this maximum spacing results in overlapping of the original spectrum with its alias [94]. In this specific example, the maximum pilot spacing is $K=7$. For $K=8$, an error floor occurs at around $\mathrm{BER}=5 \times 10^{-3}$ as a result of spectrum aliasing. Although the higher value of $\alpha$ gives better PAPR, it however produces worse BER performance for large value of $\alpha$. When $\alpha=0$, there is no interference from the data frequencies at the pilot locations, resulting in better channel estimates. Hence, there exists a fundamental trade-off between the channel estimation performance and the BER performance.

\subsection{Summary}

Two techniques of in-band FDM pilot multiplexing have been presented: FDSPT, where pilots are superimposed on scaled data-carrying tones, and FET, where groups of data carrying tone are shifted for multiplexing of pilot tones. It was shown that 
both techniques yield larger envelope variations of the SC signal. However, the SC signals with FDSPT and FET have still lower PAPR than that of an OFDM signal, resulting from the smaller number of random data symbols contributing to the peak envelope variations. The application of FDSPT depends on the pilot overhead ratio and the time variation of the channel. Using FDM pilots in the system provides the option of channel estimation in frequency domain at the expense of slightly increasing the PAPR. Also, frequency domain signal generation and pilot multiplexing facilitates flexible and efficient assignment of signals to available spectrum. Table 4.1 summarizes the advantages and disadvantages among different pilot arrangement schemes, where higher PAPR means more power backoff. The IBSDFE equalizer with

Table 4.1: Comparison of Different Pilot Arrangement Schemes

\begin{tabular}{|c|c|c|}
\hline & Advantages & Disadvantages \\
\hline TDM & $\begin{array}{c}\text { No BER degradation, } \\
\text { low PAPR }\end{array}$ & $\begin{array}{c}\text { Higher overhead, } \\
\text { less spectrum flexibility }\end{array}$ \\
\hline FET & $\begin{array}{c}\text { No BER degradation, } \\
\text { good spectrum flexibility }\end{array}$ & $\begin{array}{c}\text { Higher PAPR, } \\
\text { slightly higher overhead }\end{array}$ \\
\hline FDSPT & $\begin{array}{c}\text { Least overhead, } \\
\text { good spectrum flexibility }\end{array}$ & $\begin{array}{c}\text { slightly higher PAPR, } \\
\text { BER degradation }\end{array}$ \\
\hline
\end{tabular}

decision directed interference cancellation technique at the pilot frequency indices $\Omega$ is almost equivalent to a version of IBSDFE in which the forward filter is after the feedback and subtraction operation. The only differences are (1) in IBSDFE with interference cancellation, hard decisions are subtracted, instead of soft decisions; and (2) in IBSDFE, the received frequency samples at $\Omega$ are forced to zero (or $\alpha=0$ ) for multiplexing of pilot tones. 


\section{Chapter 5}

\section{Channel Estimation using FDM Pilots for Full Bandwidth Systems}

In this chapter, we propose two non-iterative frequency domain channel estimators and one frequency domain iterative decision-directed channel estimator (IDDCE) using the FDM pilots, described in the previous chapter, for full bandwidth systems in time-varying frequency selective fading channels. In this thesis, we define full bandwidth systems as the systems in which a single user occupies the whole available signal bandwidth. In the next chapter, we consider channel estimation for systems in which a single user occupies only part of the non-contiguous available signal bandwidth ${ }^{\mathbf{1}}$. The effects of mis-matched knowledge of the maximum delay spread and the speed of the user terminal, when generating the Wiener interpolation filter taps, is also investigated. Moreover, we compare the performance of non-iterative and iterative channel estimators for SC systems using TDM and FDM pilot schemes. We also compare the performance of SC systems with that of coded OFDM systems with FDM pilots. The FER performance of SC systems with FDSPT pilots using the IBSDFE equalizer with iterative decision-directed interference cancellation technique is also included.

\footnotetext{
${ }^{1}$ It is necessary to separate the discussion of channel estimation techniques for full bandwidth systems and chunk-based systems since different pilot schemes and channel estimation techniques maybe be required for the two systems. For example, in chunk-size systems, the number of pilots is limited by the size of a chunk, which affects the design of the corresponding channel estimator.
} 


\subsection{Introduction}

Using FDM (FDSPT and FET) pilots, we first propose two non-iterative hybrid channel estimation techniques, with DFT-based channel estimator in the frequency direction and linear least square line fitting (LLSLF) or linear Wiener filtering in the time direction of the time-frequency plane. Our motivation is to design a low complexity non-iterative channel estimator for uplink SC systems in time-varying frequency selective fading channels, comparing it later to more complex iterative approaches. The implementation complexity of a DFT channel estimation is usually low, resulting from the efficient implementation of the FFT and requiring no channel statistics. However, in terms of performance of the channel estimator, the non-iterative channel estimators may not be adequate in some applications. Hence, to further improve the performance of the channel estimation, we propose a low complexity iterative decision-directed channel estimator (IDDCE). The proposed IDDCE is a modified version of the iterative channel estimation (ICE) for OFDM systems, originally proposed in [56] for OFDM systems and briefly described in Sec. 2.6.4. The averaged MSE performance of the proposed non-iterative and iterative channel estimators is included to evaluate their performance.

Since Doppler spectrum and channel delay profile are usually a priori unknown, mis-matched channel statistics are assumed when generating the Wiener filter taps. In this chapter, we also evaluate the effects of using mis-matched channel statistics, such as the maximum channel delay spread and the vehicle speed. Moreover, to evaluate the effectiveness of our proposed FDM pilot schemes, we compare the performance of SC systems using FDM pilots with that using TDM pilots. We also compare with the coded OFDM systems using FDM pilots. The comparison is based on required power backoff of the high power amplifier (HPA), the CP overhead, pilot overhead and the FER performance. 


\subsection{Non-Iterative Channel Estimation for SC Sig- nals with FDM Pilots}

The channel estimation techniques for OFDM systems, described in Sec. 2.6 .2 and Sec. 2.6.3, can be used for channel estimation of SC signals with FDM pilots. The channel estimation is accomplished by estimating the channel frequency response (CFR) at the pilot frequencies $(\ell \in \Omega)$ and then interpolating among these estimated frequencies to obtain the channel estimates for the whole block, using non-iterative channel estimators, such as a DFT-based channel estimator ${ }^{2}$, a cascaded $2 \times 1 \mathrm{D}$ Wiener filtering or iterative decision-directed channel estimation techniques. In addition to the cascaded $2 \times 1 \mathrm{D}$ Wiener filtering used for OFDM systems, in this section we propose two hybrid channel estimators ${ }^{3}$ (FFT-LLSLF or FFT-Wiener estimators) and one frequency domain iterative decision directed channel estimation (IDDCE) technique for SC systems (to be described in Sec. 5.3). For FET, (2.44) can be used to find the initial raw estimates of the channel frequency response at $\ell \in \Omega$. For FDSPT the initial estimates can be calculated as

$$
\begin{aligned}
\hat{H}_{k, \ell} & =\frac{Y_{k, \ell}}{\beta P_{k, \ell}} \\
& =H_{k, \ell}+V_{k, \ell}^{\prime \prime}, \quad k \in \Psi \text { and } \ell \in \Omega
\end{aligned}
$$

where

$$
V_{k, \ell}^{\prime \prime}=\frac{\alpha A_{\ell}}{\beta P_{k, \ell}}+\frac{V_{k, \ell}}{\beta P_{k, \ell}} .
$$

From (5.2), we see that when $\alpha=0$ and $\beta=1$, the initial estimates using FDSPT are the same as that of using FET. In other words, the performance of the non-iterative channel estimators using FET and FDSPT will be the same when $\alpha=0$ and $\beta=1$.

\subsubsection{Hybrid Channel Estimator: FFT-LLSLF}

This hybrid channel estimator uses a DFT-based channel estimator in the frequency direction and linear LS line fitting (LLSLF) [52] in the time direction, assuming that

\footnotetext{
${ }^{2}$ We sometimes call a DFT based channel estimator an FFT channel estimator in this thesis.

${ }^{3}$ Hybrid means that the estimators used in the time and frequency directions are not the same.
} 
the channel is linearly time-varying. The motivation for this hybrid estimator is its low complexity and requiring no channel and noise statistics. The obvious price to pay for low complexity is the performance of the channel estimators. The linear assumption in the time direction requires high pilot overhead when the maximum Doppler frequency $f_{\text {Dmax }}$ is high.

\subsubsection{Hybrid Channel Estimator: FFT-Wiener}

To improve the performance in the time direction (at the expense of slightly higher complexity), while using low complexity DFT-based channel estimation in the frequency direction the other proposed hybrid channel estimator uses the DFT-based channel estimation in the frequency direction and Wiener filtering in the time direction. The objective is to reduce the computational complexity involved in Wiener filtering in the frequency direction as usually $M>N_{B}$, when on-line calculation of Wiener filter taps is required. Note that if the Wiener filter taps are pre-stored, the hybrid estimator has slightly higher computational complexity than that of using $2 \times 1 \mathrm{D}$ Wiener filtering.

\subsection{Iterative Channel Estimation for SC Signals with FDM Pilots}

To further improve the performance of the channel estimator, the tentative symbol decisions can be treated as extra pilot symbols and fed back to the channel estimator to re-estimate the entire time and frequency grid. We want to consider a low-complexity IDDCE for SC systems, inspired by the two-dimensional iterative decision-directed channel estimation for OFDM system (see Sec. 2.6.4). From Fig. 2.12 we know that tentative decisions can be used to improve the performance of the non-iterative cascaded 1D-FIR Wiener filtering. The next question to ask is whether we can directly apply the frequency domain iterative decision-directed channel estimator for OFDM systems for SC systems? The answer is no since the envelope of the frequency response 
of the decisions is not constant, resulting in the issue of noise enhancement when calculating the raw estimates of the CFR. A low complexity thresholding technique is used to overcome the issue of noise enhancement. We next describe our proposed low complexity frequency domain IDDCE for SC systems using FDM Pilots.

\subsubsection{Proposed Low Complexity Frequency Domain IDDCE}

We modify the frequency domain IDDCE described in Sec. 2.6.4, originally designed for OFDM systems, for SC systems with FDM pilots ${ }^{4}$. Instead of using the LLR of the coded symbols from the output of an APP decoder, the hard decisions either from the equalizer output or the decoder output are used for low complexity. This is suboptimum, but as we will see from the simulation results, it gives performance close to the case of known CFR. Fig. 5.1 shows the structure of the proposed frequency domain IDDCE for SC systems with or without involving the decoder. The

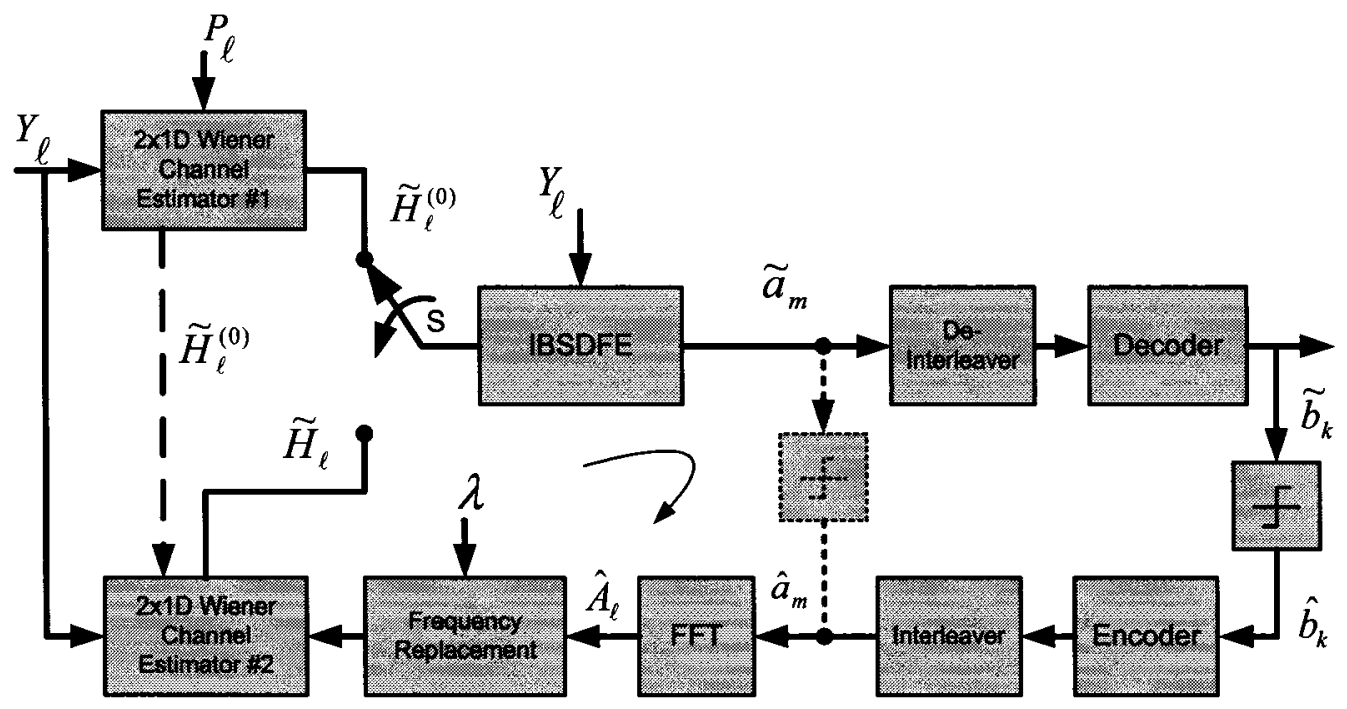

Figure 5.1: Block Diagram of the Proposed Frequency Domain Iterative Decision-Directed Channel Estimator for SC Systems with or without Decoder

dotted hard decision block is used when the decoder is not involved, ignoring the interleaver/de-interleaver and encoder/decoder. During the initial channel estima-

\footnotetext{
${ }^{4}$ Although we only consider SC systems with FDM pilots, the proposed IDDCE technique can also be used for SC systems using TDM pilots as long as the initial channel estimates are available.
} 
tion using a cascaded $2 \times 1 \mathrm{D}$ Wiener filter $\# 1$, the switch $S$ is in the upper position. The initial estimated CFR $\tilde{H}_{\ell}^{(0)}$ is then used by the IBSDFE, producing the soft decision of the transmitted data symbol $\tilde{a}_{m}$. If the decoder is not involved, the hard decision of $\tilde{a}_{m}$, denoted as $\hat{a}_{m}$, is then used to calculate $\hat{A}_{\ell}$ by taking the FFT of $\hat{a}_{m}$. If the decoder is involved, after de-interleaving and soft Viterbi decoding, the hard decisions of the decoder output $\hat{b}_{k}$ are re-encoded and interleaved to obtain the hard decisions of the transmitted data symbols $\hat{a}_{m}$. Given the received frequency sample $Y_{\ell}$ and $\hat{A}_{\ell}$, our next task is to find the raw estimates of the channel frequency responses for the Wiener filtering process, given as $[40,52]$

$$
\hat{H}_{\ell}=\frac{Y_{\ell}}{\hat{A}_{\ell}}, \quad \ell=0,1, \ldots, M-1
$$

where $\hat{A}_{\ell}$ is the FFT of $\hat{a}_{m}$, given as

$$
\hat{A}_{\ell}=\frac{1}{\sqrt{M}} \sum_{m=0}^{M-1} \hat{a}_{m} e^{-\frac{j 2 \pi m \ell}{M}}, \quad \ell=0,1, \ldots, M-1 .
$$

Note that we ignore the block index $k$ for simplicity.

\subsubsection{Issue of Noise Enhancement}

Using the Central Limit Theorem [91], $\hat{A}_{\ell}$ in (5.4) can be approximated as a Gaussian random variable with zero mean and unit variance. From (5.3), assuming all the decisions from the equalizer are correct, the variance of $\hat{H}_{\ell}$ given $\hat{A}_{\ell}$ can be found as

$$
\hat{\sigma}_{\ell}^{2}=E\left\{\left|H_{\ell}-\hat{H}_{\ell}\right|^{2}\right\}=\frac{\sigma^{2}}{\left|\hat{A}_{\ell}\right|^{2}}
$$

where $\sigma^{2}$ is the noise variance. It is obvious that from (5.5), when $\left|\hat{A}_{\ell}\right|$ is small, noise enhancement makes interpolation of $\hat{H}_{\ell}$ to find $\tilde{H}_{\ell}$ difficult.

\subsubsection{Optimum Technique: MMSE Estimator}

Using (5.3) to find the LS (or raw) estimates for the second Wiener filter can result in noise enhancement. Our first approach to overcome this issue is to use an optimum MMSE estimator to find the raw estimates. Assuming that all the decisions are 
correct so that $\hat{A}_{\ell}=A_{\ell}$ for $\ell=0,1, \cdots, M-1$, the received frequency samples can be written, in matrix form, as

$$
\mathbf{Y}=\hat{\mathbf{A}} \mathbf{H}+\mathbf{V}
$$

where $\hat{\mathbf{A}}$ is a diagonal matrix with $\left[\hat{A}_{0}, \hat{A}_{1}, \cdots, \hat{A}_{M-1}\right]^{T}$ being the main diagonal elements, $\mathbf{H}=\left[H_{0}, H_{1}, \cdots, H_{M-1}\right]^{T}$ is the CFR and $\mathbf{V}=\left[V_{0}, V_{1}, \cdots, V_{M-1}\right]^{T}$ is the vector of noise frequency samples. Since $\mathbf{Y}$ and $\mathbf{H}$ are jointly complex Gaussian, the MMSE (conditional mean) estimator for $\mathbf{H}$, given $\mathbf{Y}$ and $\hat{\mathbf{A}}$, is given as [52, pp. 533],

$$
\hat{\mathbf{H}}^{\prime}=\mathbf{C}_{H H} \hat{\mathbf{A}}^{H}\left(\hat{\mathbf{A}} \mathbf{C}_{H H} \hat{\mathbf{A}}^{H}+\mathbf{C}_{V V}\right)^{-1} \mathbf{Y}
$$

where ${ }^{H}$ denotes the Hermitian transpose, $\mathbf{C}_{H H}$ and $\mathbf{C}_{V V}=\sigma^{2} \mathbf{I}$ are the covariance matrix of the CFR and the noise samples, respectively. Inspecting (5.7), we notice that to obtain $\hat{\mathbf{H}}^{\prime}$ not only requires the knowledge of the covariance matrix of the CFR $\mathbf{C}_{H H}$ but also the DFT of the data decision $\hat{\mathbf{A}}$, which varies from block to block. This means that we need to calculate the filter taps on-line for each block. Note that by dividing $\mathbf{Y}$ into smaller size vectors, a smaller size of matrix inversion can be used to reduce the complexity, at the expense of slight performance degradation, as shown in Fig. 5.4. (5.7) provides an optimum solution with relatively high complexity for practical implementation of the proposed iterative channel estimator.

\subsubsection{Sub-optimum Low Complexity Technique: The Fre- quency Replacement Algorithm}

To overcome the issue of high complexity provided by (5.7) and the issue of noise enhancement, we propose to replace the frequency channel estimates at noise enhanced frequencies with the initial channel frequency estimates (or the channel estimates in the previous iteration) using a threshold $\lambda$. We called this method frequency replacement (FR). The mixed LS estimates using tentative decision tones and the channel frequency estimates in the previous iteration are given as

$$
\hat{H}_{\ell}^{\prime}= \begin{cases}\frac{Y_{\ell}}{\hat{A}_{\ell}}, & \left|\hat{A}_{\ell}\right|>\lambda \\ \tilde{H}_{\ell}^{\text {prev }}, & \left|\hat{A}_{\ell}\right| \leq \lambda\end{cases}
$$


where $\ell=0,1, \ldots, M-1$ and $\tilde{H}_{\ell}^{\text {prev }}$ is the channel frequency estimates in the previous iteration. Since $\hat{A}_{\ell}$ can be modeled as a Gaussian random variable, $\left|\hat{A}_{\ell}\right|$ has a Rayleigh probability density function. Hence, the probability of replacing the frequencies can be calculated as

$$
\operatorname{Pr}\left\{\left|\hat{A}_{\ell}\right| \leq \lambda\right\}=1-e^{-\lambda^{2}}
$$

where we assume $E\left\{\left|\hat{A}_{\ell}\right|^{2}\right\}=1$. Assuming that $\operatorname{Pr}\left\{\left|\hat{A}_{0}\right| \leq \lambda\right\}=\operatorname{Pr}\left\{\left|\hat{A}_{1}\right| \leq \lambda\right\}=$ $\ldots=\operatorname{Pr}\left\{\left|\hat{A}_{M-1}\right| \leq \lambda\right\}$, the average number of frequencies replaced for a particular block can be calculated as

$$
N_{F R}=M\left(1-e^{-\lambda^{2}}\right)
$$

where $M$ is the number of frequencies per block. It should be noted that the value of the threshold $\lambda$ determines the number of frequencies to be replaced. When $\lambda$ is too low, noise enhancement persists. In the other hand, if $\lambda$ is too high, most of the channel frequency estimates in the previous iteration will be re-used, resulting in similar performance as that of the previous channel estimates. Assuming that $E\left\{\left|\hat{A}_{\ell}\right|^{2}\right\}=1$, the threshold value should be chosen between 0 and 1, i.e. $0<\lambda<1$. In Section 5.3 .6 of this thesis, the best threshold value $\lambda$ is found by simulation. The FR algorithm is a low complexity technique, as no matrix inversion is required.

\subsubsection{Wiener $2 \times 1 \mathrm{D}$ Filtering of Raw Estimates}

Using the frequency channel estimates from either (5.7) or (5.8), another $2 \times 1 D$ Wiener filter (similar to the one used for initial channel estimation) with the smallest pilot distance in both time and frequency direction (denoted as $D_{t}$ and $D_{f}$, respectively), i.e. $D_{f}=D_{t}=1$, is used to refine the channel estimates for the whole frame. Note that denser pilots for Wiener filtering in general gives better smoothing performance. In this case, we use the sixteen nearest pseudo pilots for smoothing in the frequency direction, while we use $Q$ (number of blocks per frame) pseudo pilots for smoothing in the time direction. Switch $S$ in Fig. 5.1 is then in the lower position, allowing the improved channel frequency estimates to be used by the IBSDFE and 
forming a closed loop for the next iteration. The difference between the $2 \times 1 D$ Wiener filter \#1 and $2 \times 1 D$ Wiener filter \#2 in Fig. 5.1 is that the values of $D_{f}$ and $D_{t}$ in $2 \times 1 \mathrm{D}$ Wiener filter \#2 is always 1 , while the corresponding values for $2 \times 1 D$ Wiener filter \#1 is $D_{t}=(Q-1)>1$ and $D_{f}=K>1$. In our simulation, we assume $Q=12$ and $K=4$ and the pilots are added in the first and the last block of the frame.

\subsubsection{Determination of the Best Value of $\lambda$}

We first determine the best value of $\lambda$ by simulating a SC system with IDDCE using different values of $\lambda$ over a wide range of SNR. Parameter Set 2-B (see Table 2.3 and Table 2.4) is used for the simulation and channel realizations for 2000 frames are simulated. Fig. 5.2 shows the averaged MSE of the IDDCE using FR algorithm with various values of $\lambda$ at $\mathrm{SNR}=5,10$, and $20 \mathrm{~dB}$. The averaging is taken over the whole estimated time-frequency plane, given as

$$
M S E=\frac{1}{M N_{B}} \sum_{k=0}^{N_{B}-1} \sum_{\ell=0}^{M-1}\left|H_{k, \ell}-\tilde{H}_{k, \ell}\right|^{2}
$$

where $M$ is the number frequencies and $N_{B}=12$ is the number of blocks per frame. For all three SNR values, the best (in terms of smallest MSE by observation) values for FR algorithm was found to be around $\lambda=0.3$. With $\lambda=0.3$ and using (5.9), the probability of replacing a particular frequency using FR algorithm is

$$
\operatorname{Pr}\left\{\left|\hat{A}_{\ell}\right| \leq \lambda\right\}=1-e^{-(0.3)^{2}}=8.6 \%
$$

and the average number of frequencies replaced in a blocked is, using $(5.10), N_{F R}=$ $M \times 8.6 \% \approx 89$. This means that we are using most $(91.4 \%)$ of the tentative decision tones for channel estimation for the next iteration.

\subsection{Performance Evaluation}

The averaged MSE of the channel estimates and FER are used to evaluate the performance of the proposed channel estimators and the system performance with channel 


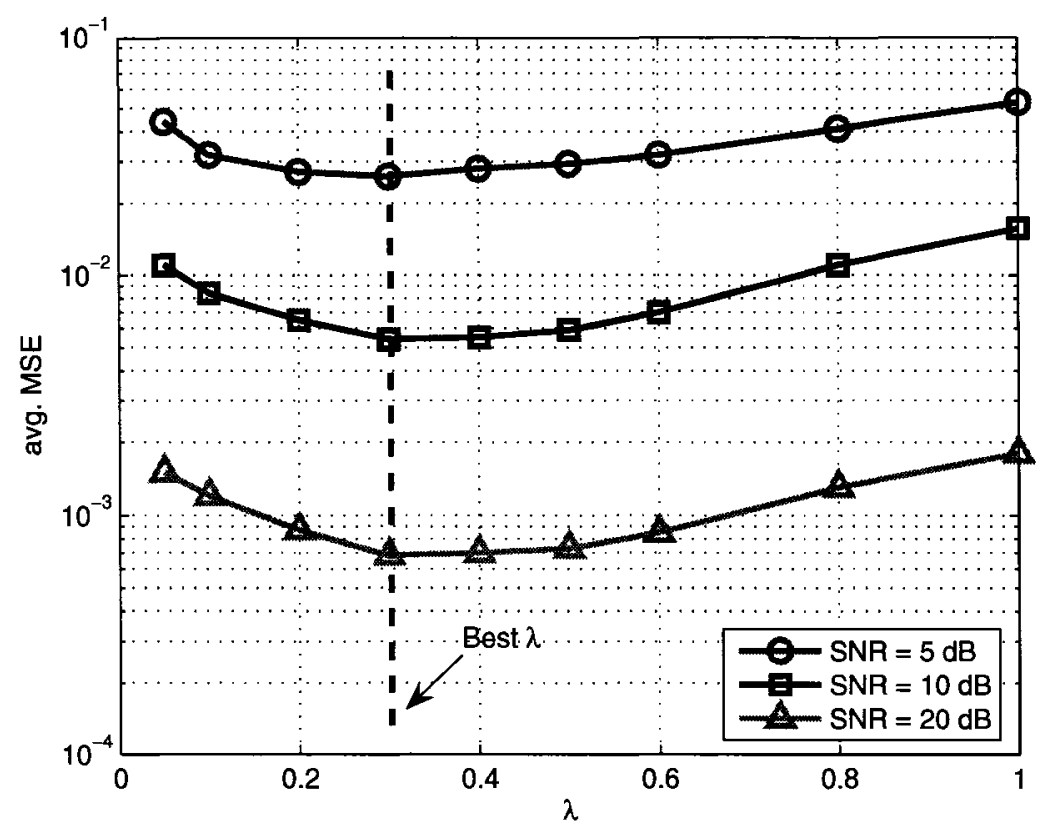

Figure 5.2: Effects of the Threshold Values for IDDCE (Parameter Set 2-B)

estimation, respectively. Moreover, we compare the performance of non-iterative and iterative channel estimators for SC systems using TDM and FDM pilot schemes in terms of pilot overhead, CP overhead, the required power backoff for the HPA, and the FER. We also compare the performance of SC systems with that of OFDM systems with the same parameter set.

\subsubsection{Averaged MSE}

Fig. 5.3 depicts the averaged MSE for the non-iterative channel estimators. (5.11) is used to obtain the averaged MSE of the channel estimates. Parameter Set 2-A is used for the simulation and channel realizations for 2000 frames are used to generate the MSE results. A ' 1 ' represents pilots are present in the corresponding block, while a ' 0 ' means no pilots are placed in the corresponding block. $2 \times 1 \mathrm{D}$ Wiener means cascaded $2 \times 1 \mathrm{D}$ Wiener filters. $I_{f}, I_{t}$ and $I$ represent the number of filter taps in the frequency direction, the time direction and the time-frequency plane, respectively. For a fair comparison, we fix the total number of pilots in a frame at 208 pilots per frame so 


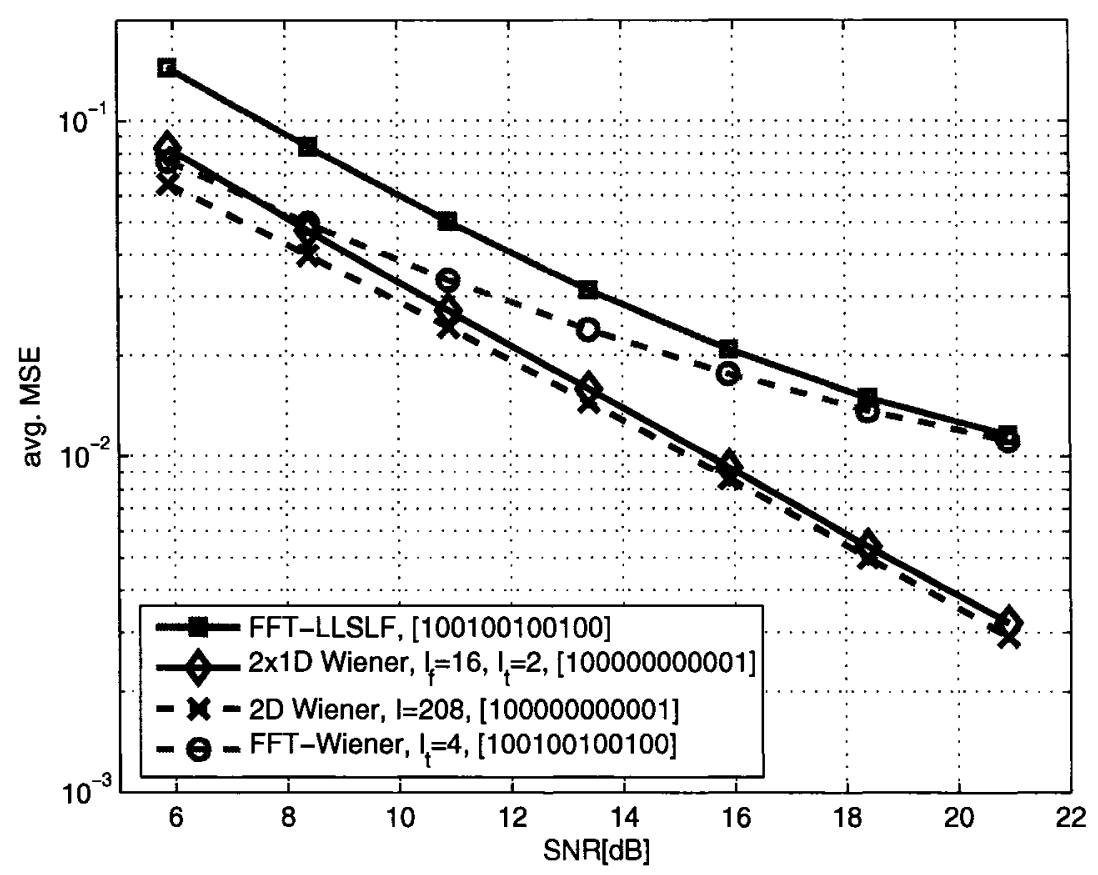

Figure 5.3: MSE Performance of Different Non-Iterative Channel Estimators for Parameter Set $2-A$

that the pilot overhead is $1 / 24=4.17 \%$. We found that two more blocks with pilots are required when using FFT-LLSLF estimator. The FFT-LLSLF channel estimator appears as the worst estimator among the three estimators. This is expected, as the channel and noise statistics are not incorporated into the FFT estimator. The hybrid estimator improves the performance for low SNR, compared with that of the FFT-LLSLF estimator. It is obvious the Wiener filtering outperforms the LLSLF estimation in the time direction. For high SNR ( $>16 \mathrm{~dB})$, the MSE caused by the FFT estimator dominates the MSE caused by the white noise, resulting in a similar performance as that of using LLSLF. At low SNR region $(\leq 10 \mathrm{~dB})$, the hybrid estimator provides a low computational complexity solution for the cascaded $2 \times 1 \mathrm{D}$ Wiener filtering. The averaged MSE for the linear optimum 2D Wiener filtering is also included for reference. As concluded in [81], there is not much gain, in terms of averaged MSE, using all the pilots for estimation (see the curves with a cross (2D 
Wiener $)$ and a diamond $(2 \times 1 \mathrm{D}$ Wiener $)$ in Fig. 5.3, which are almost coincident).

Fig. 5.4 depicts the averaged MSE for the IDDCE with FET pilots. Parameter Set 2- $B$ is used for the simulation. The averaged MSE performance curve for noniterative $\mathrm{W} 2 \times 1 \mathrm{D}$ and a frame with all Chu pilots are also included for comparison. The MSE performance of using all Chu pilots can be considered as the optimum MSE performance for IDDCE since there exists no noise enhanced raw estimates and decision errors. The legend 'H-ICE1, $\lambda=0.3$ ' means hard decision iterative channel estimation with 1 iteration and FR algorithm with $\lambda=0.3$ is used. The ' $\mathrm{C}$ ' in the legend ' $\mathrm{H}-\mathrm{ICE} 1-\mathrm{C}, \lambda=0.3$ ' means that decoding is included in the channel estimation process. We see from Fig. 5.4 that the IDDCE with FR algorithm in general gives MSE performance improvement over the non-iterative cascaded $2 \times 1 \mathrm{D}$ Wiener filter. However, in the low SNR region from $5 \mathrm{~dB}$ to $12 \mathrm{~dB}$, the MSE performance of the IDDCE with FR algorithm without involving the decoder is degraded as a result of decision errors. The IDDCE using the FR algorithm with re-encoding improves the MSE performance at the low SNR region. The use of the optimum MMSE estimator to obtain the raw estimates for the second Wiener filtering gives MSE performance close to that of using all pilots in the high SNR region $(>12 \mathrm{~dB})$, where decision errors

are small. On the other hand, in the low SNR region $(\leq 10 \mathrm{~dB})$, the low complexity IDDCE using FR algorithm with a threshold gives comparable MSE performance to that of using the optimum MMSE estimator. Moreover, when a smaller size of matrix inversion, e.g. $16 \times 16$, is used, the MSE performance is similar to that of using the proposed low complexity IDDCE.

\subsubsection{Frame Error Rate (FER)}

Fig. 5.5 and Fig. 5.6 depicts the FER of the coded systems with TDM, FET and FDSPT pilots using different non-iterative channel estimators, where ' $\mathrm{W}$ ' stands for Wiener filtering. Parameter Set 2-A (see Table 2.3 and Table 2.4) is used for the simulation and frequency domain IBSDFE equalization (see Sec. 4.3) is used. First 


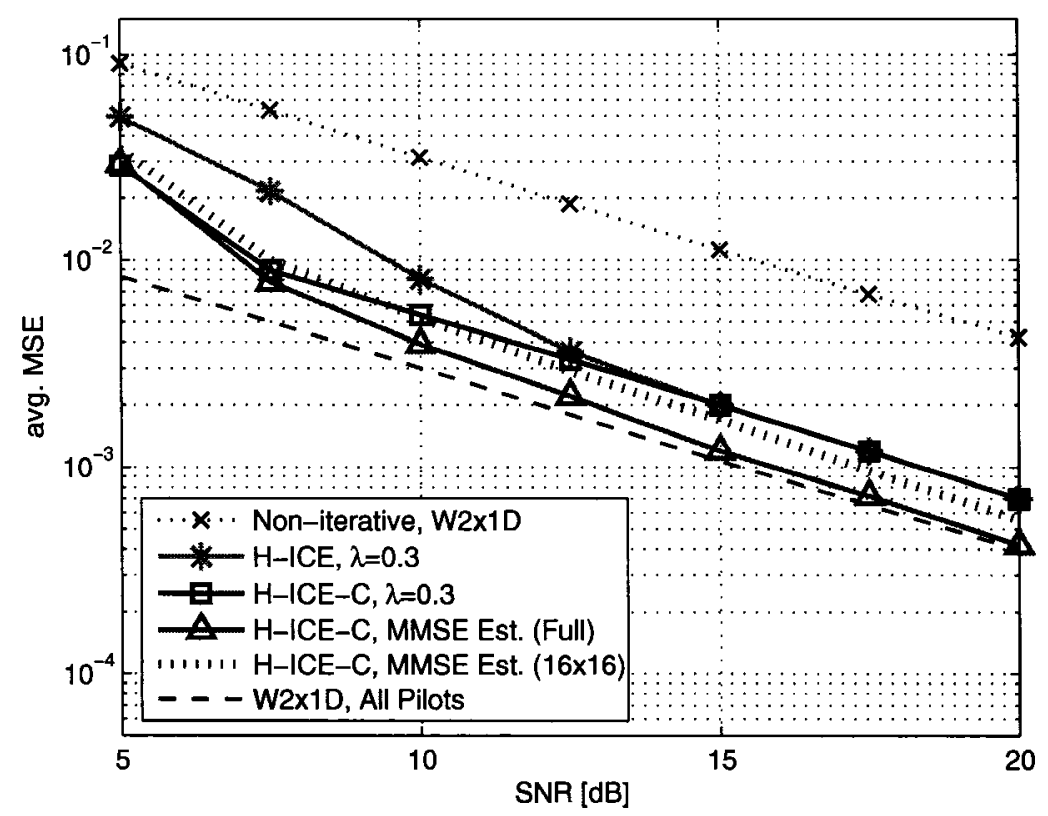

Figure 5.4: MSE Performance of IDDCE using FET Pilots for Parameter Set 2-B

consider Fig. 5.5. The FFT-Wiener channel estimator (see Sec. 5.2.2) has about 0.5 $\mathrm{dB}$ improvement at FER $=10^{-2}$, compared with that of an FFT-LLSLF estimator. The FET and TDM have similar performance at FER $=10^{-2}$, while FDSPT loses about $1 \mathrm{~dB}$ to both TDM and FET. Consider Fig. 5.6 for Wiener filter interpolation. In general, the performance at $\mathrm{FER}=10^{-2}$ improves about $0.6 \mathrm{~dB}$ over that using the FFT-LLSLF estimator. The performance for the two-dimensional (2D) Wiener estimator is also included for reference. Notice that the performance degradation using the FFT-Wiener estimator is only $0.2 \mathrm{~dB}$ relative to that of a cascaded $2 \times 1 \mathrm{D}$ Wiener estimator.

Fig. 5.7 shows the frame error rate (FER) performance of the coded SC systems with FDSPT $(\alpha=0)$ and FET pilots using the proposed IDDCE technique using the FR algorithm with $\lambda=0.3$. Note that Parameter Set $2-B$ and the IBSDFE equalizer are used for the simulation. Comparing with the non-iterative $2 \times 1 \mathrm{D}$ Wiener interpolation, the IDDCE without the decoder in the loop improves about $1 \mathrm{~dB}$ for $\mathrm{FER}=10^{-2}$ and about $1.2 \mathrm{~dB}$ when decoder is included in the iteration. 


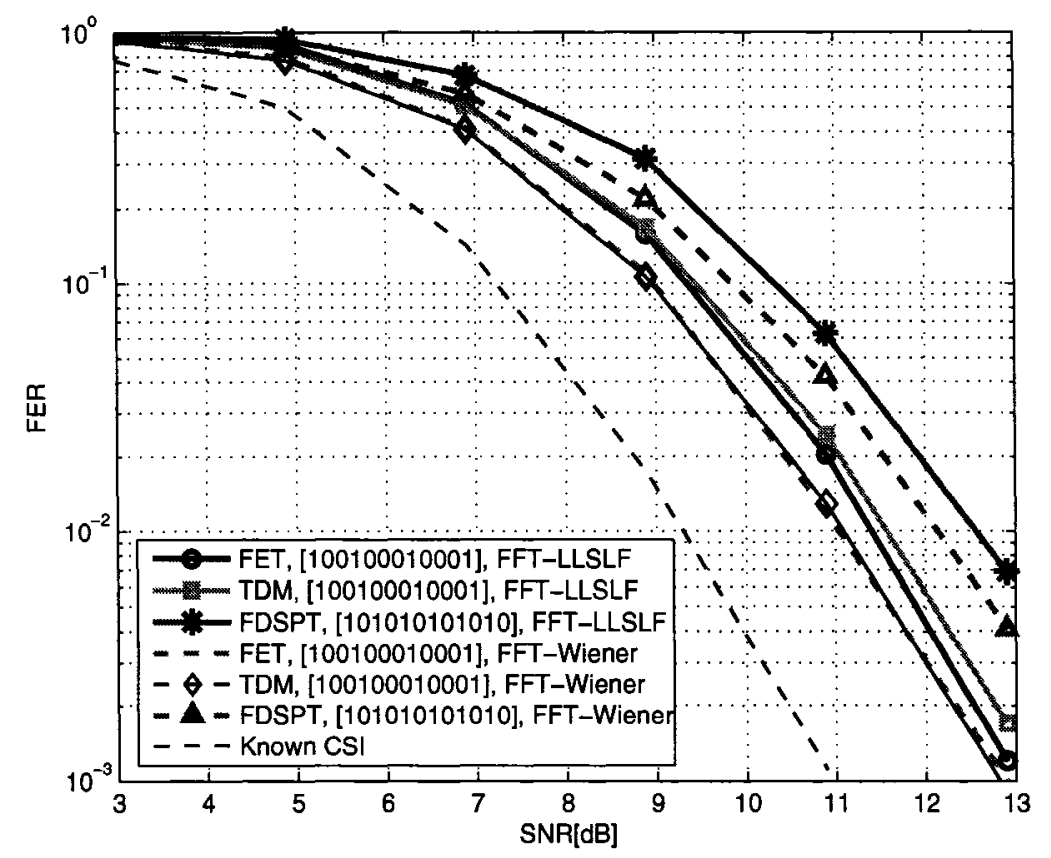

Figure 5.5: FER Performance of FDM Pilots for Coded System using IBSDFE Equalizer with Non-Iterative Channel Estimations (Parameter Set 2-A)

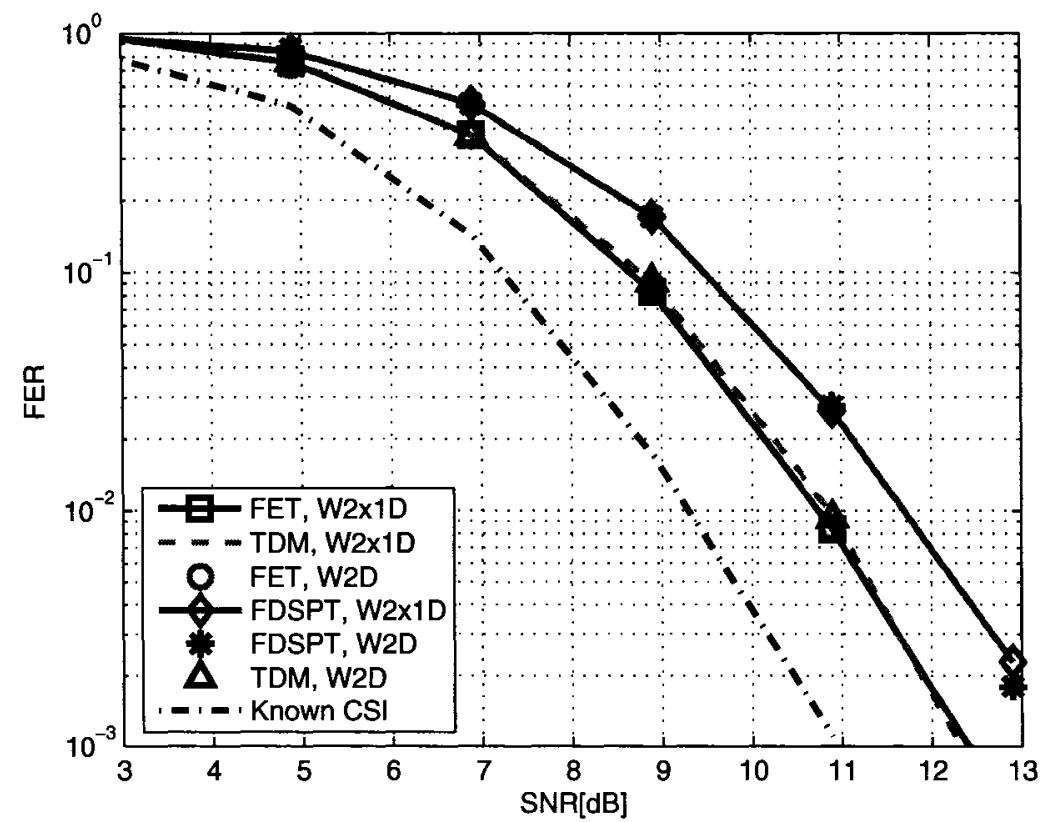

Figure 5.6: FER Performance of FDM Pilots for Coded System using IBSDFE Equalizer with Non-Iterative Channel Estimations (Parameter Set 2-A) 
This is about $0.2 \mathrm{~dB}$ away from the known CSI case. The use of IDDCE for FDSPT $(\alpha=0)$ pilots improves about $1 \mathrm{~dB}$ for $\mathrm{FER}=10^{-2}$ with the decoder in the loop and about $0.5 \mathrm{~dB}$ with the decoder not in the loop, compared with that of non-iterative $2 \times 1 \mathrm{D}$ Wiener filtering. For FDSPT $(\alpha=0)$, the FER performance is about 1 $\mathrm{dB}$, at $\mathrm{FER}=10^{-2}$, away from the known CSI case. For OFDM systems with FET pilots, shown in Fig. 5.8, the improvement for IDDCE without the decoder over the non-iterative estimator is about $0.2 \mathrm{~dB}$, while the improvement for IDDCE with the decoder is about $1 \mathrm{~dB}$, which is about $0.2 \mathrm{~dB}$ away from the known CSI case. Similar to the SC case, using FDSPT pilots for the OFDM system requires about 1 dB extra SNR at a FER of $10^{-2}$. Finally, Fig. 5.9 shows the FER for coded SC system using FDSPT $(\alpha=0)$ pilots with iterative decision-directed interference cancellation (described in Sec. 4.4). When the decoder is included, the improvement is about 0.5 $\mathrm{dB}$, which is about $0.3 \mathrm{~dB}$ from FET pilots and $0.5 \mathrm{~dB}$ away from the performance for known CSI. In other words, using the proposed iterative decision-directed interference cancellation technique, the improvement is about $0.5 \mathrm{~dB}$ at $\mathrm{FER}=10^{-2}$.

\subsubsection{Effects of Mis-Matched Delay Spread and Vehicle Speed}

Fig. 5.10 and Fig. 5.11 depict the effects of mis-matched values of maximum delay spread $\tau_{\max }$ and vehicle speed $v$ using non-iterative $2 \times 1 \mathrm{D}$ Wiener filtering and frequency domain $\operatorname{IDDCE}(\lambda=0.3)$, respectively, where different scenarios of mismatched parameters are summarized in Table 5.1. $\tau_{\text {max }}^{\prime}$ and $v^{\prime}$ are used for generating the Wiener filter taps instead of the exact values of $\tau_{\max }$ and $v$. Scenario $A$ assumes that the values of $\tau_{\max }^{\prime}$ and $v^{\prime}$ are matched to $\tau_{\max }$ and $v$, while Scenario $B$ assumes that only $\tau_{\text {max }}$ is matched and $v$ is arbitrarily assumed to be a relatively large value, such as $100 \mathrm{~km} / \mathrm{hr}$. Scenario $C$ assumes that $\tau_{\max }$ and $v$ are both mis-matched to $\tau_{m a x}^{\prime}$ and $v^{\prime}$ and they are assumed to be equal to the CP duration $T_{C P}=3.2 \mu s$ and $100 \mathrm{~km} / \mathrm{hr}$, respectively. The effect of maximum vehicle speed is insignificant as the performance degradation is about $0.1 \mathrm{~dB}$ at $\mathrm{FER}=10^{-2}$, as shown in Fig. 5.10. 


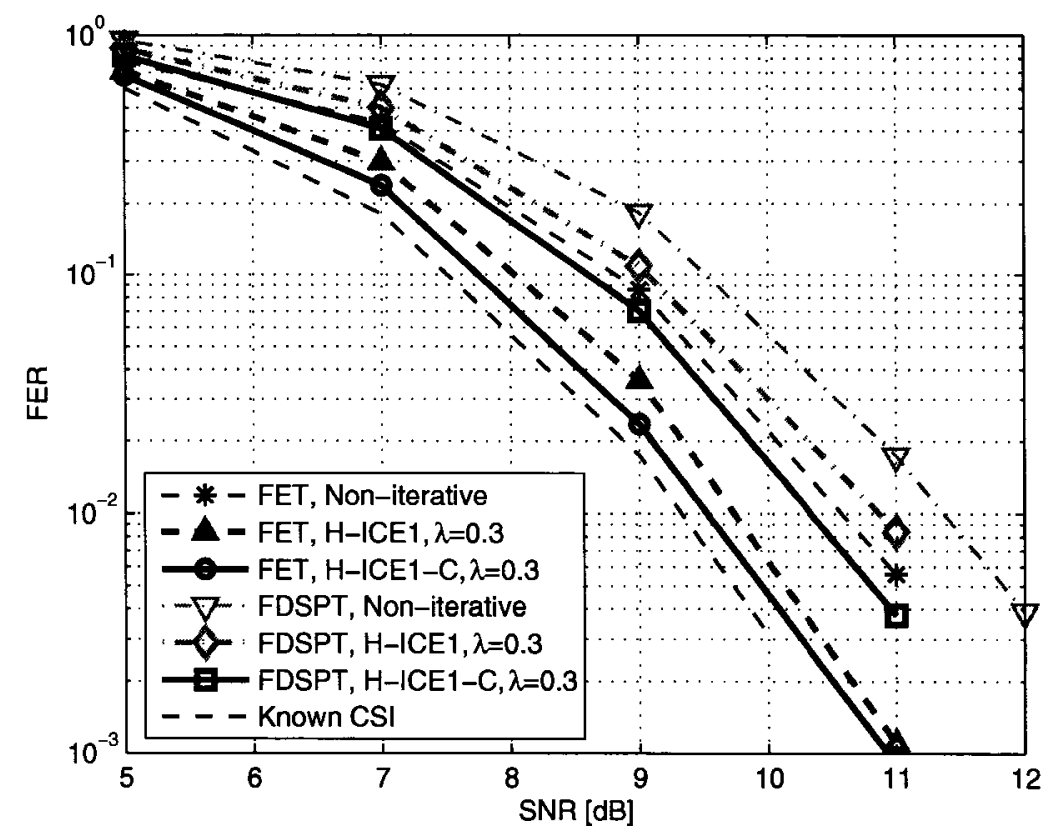

Figure 5.7: FER Performance of Coded SC Systems with FDM Pilots using the Proposed IDDCE and IBSDFE Equalizer (Parameter Set 2-B)

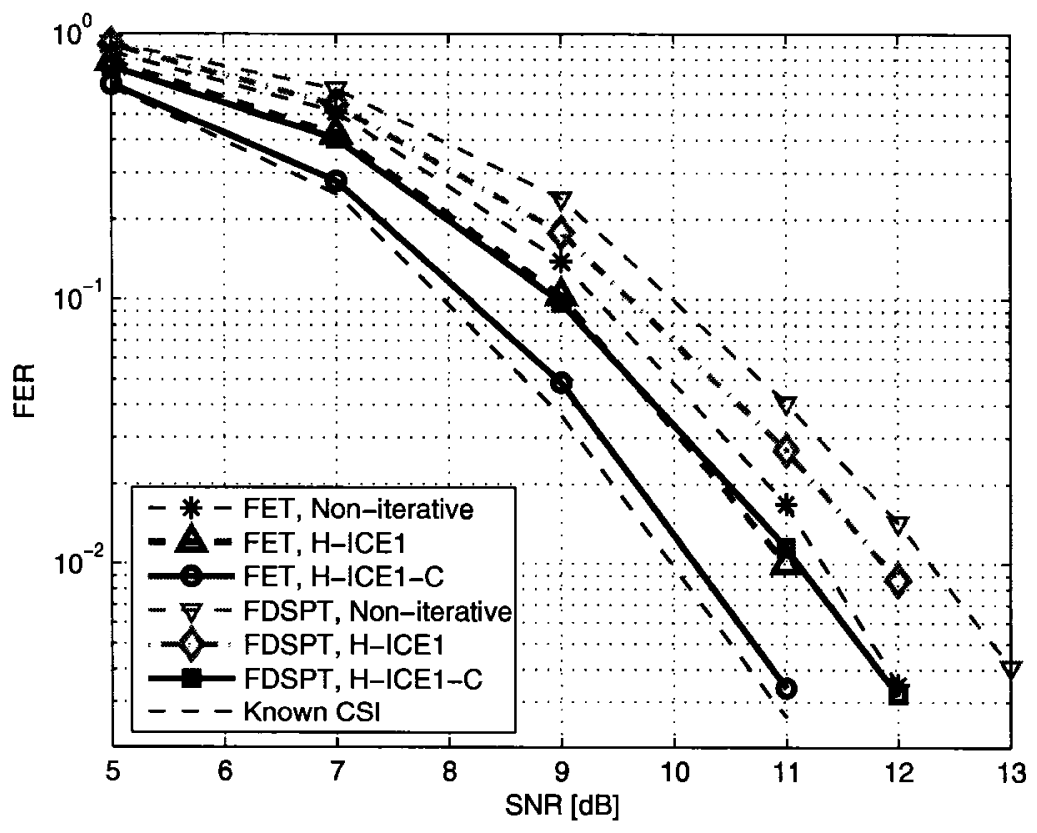

Figure 5.8: FER Performance of Coded OFDM Systems with FDM Pilots using the IDDCE Proposed in [56] and Linear Equalizer (Parameter Set 2-B) 


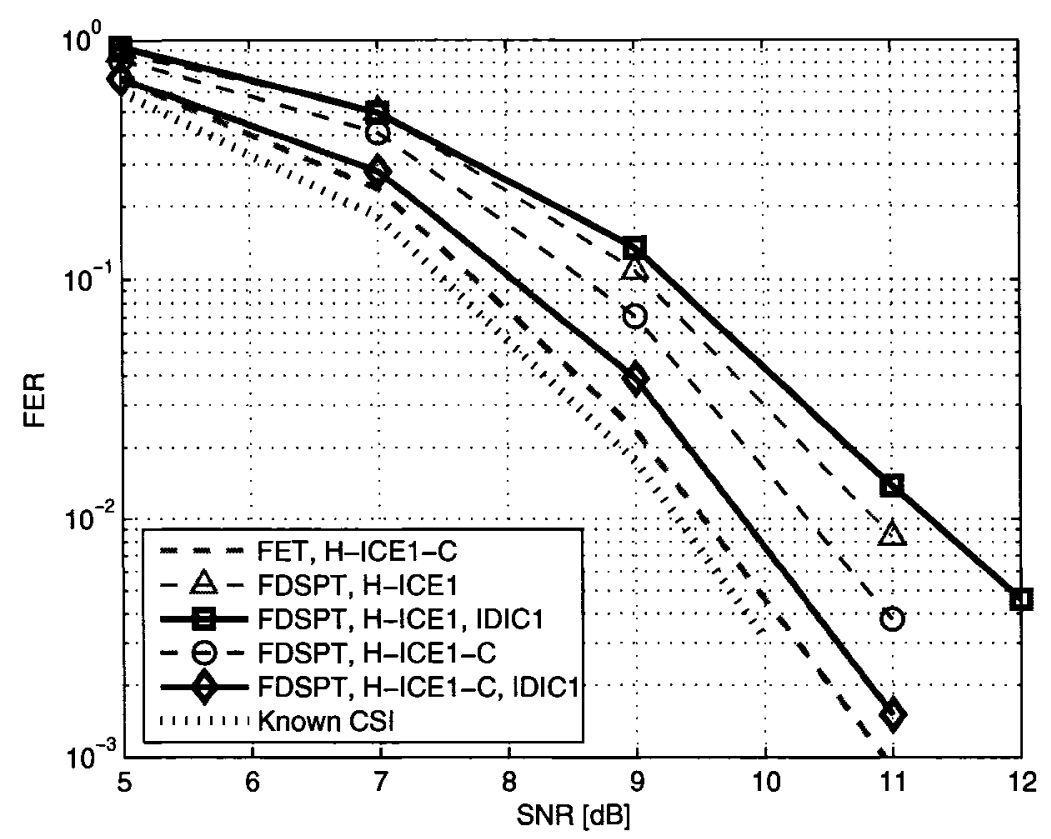

Figure 5.9: FER Performance of FDSPT Pilots for Coded SC Systems using IBSDFE Equalizer with Iterative Decision-directed Interference Cancellation, with Channel Estimation (Parameter Set 2-B)

However, the effect of maximum delay spread is more significant. The performance degradation with respect to the performance for scenario $A$ is about $1 \mathrm{~dB}$ and $0.4 \mathrm{~dB}$ at $\mathrm{FER}=10^{-2}$ for non-iterative and iterative channel estimators respectively. This implies that the estimation of the maximum delay of the channel is more crucial than the speed of the vehicle when a priori knowledge of the channel statistics for channel estimation is required. Moreover, the frequency domain IDDCE is more robust to the mis-matched effects of $\tau_{\max }$ and $v$, as shown in Fig. 5.11.

Table 5.1: Different Scenarios of Mis-Matched Parameter for Generating Wiener Filter Taps

\begin{tabular}{|c|c|c|}
\hline Scenario & $\tau_{\max }^{\prime}[\mu \mathrm{s}]$ & $v^{\prime}[\mathrm{km} / \mathrm{hr}]$ \\
\hline $\mathrm{A}$ & $\tau_{\max }=1.47$ & $v=50$ \\
\hline $\mathrm{B}$ & $\tau_{\max }=1.47$ & 100 \\
\hline $\mathrm{C}$ & $T_{C P}=3.2$ & 100 \\
\hline
\end{tabular}




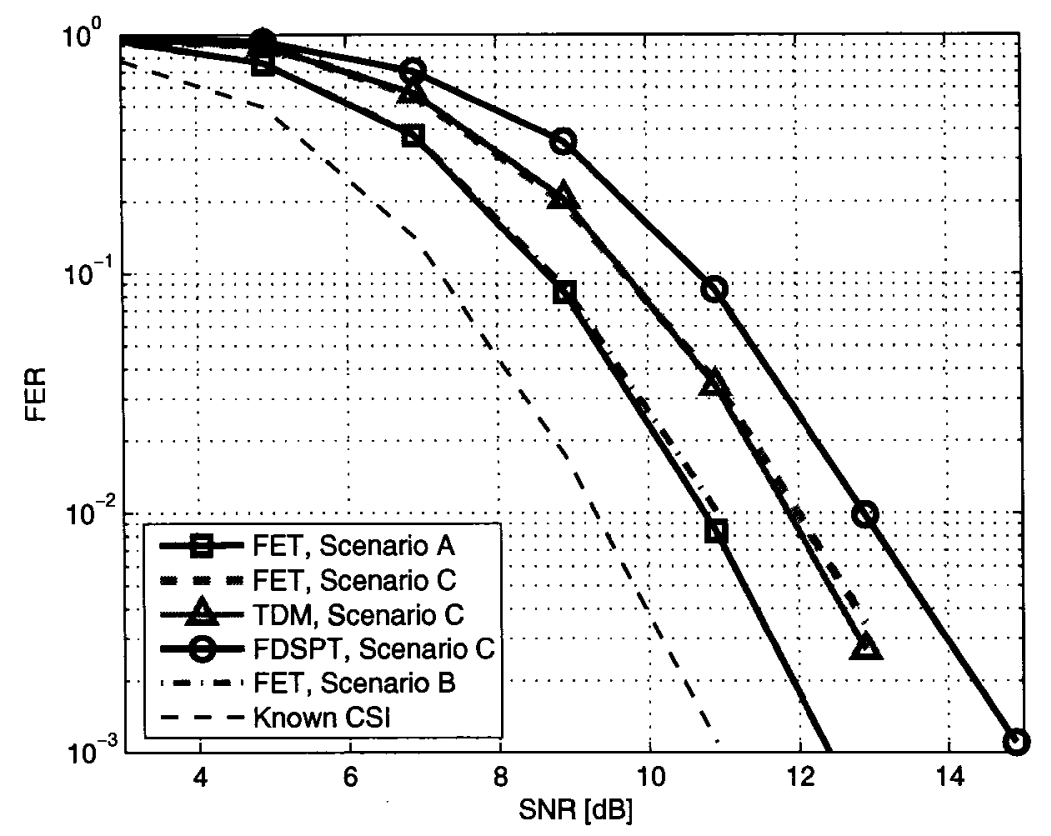

Figure 5.10: FER Performance of FDM Pilots for Coded System using IBSDFE Equalizer with Non-Iterative Channel Estimation (Parameter Set 2-A)

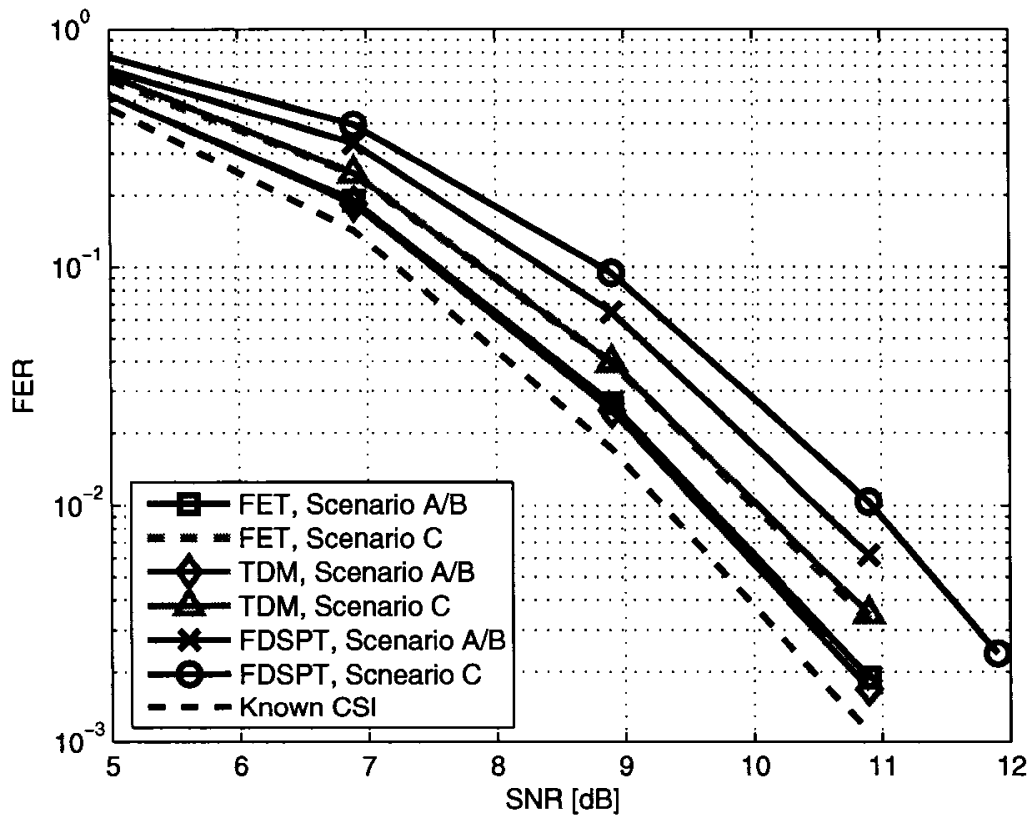

Figure 5.11: FER Performance of FDM Pilots for Coded System using IBSDFE Equalizer with Iterative Channel Estimations (Parameter Set 2-A) 


\subsection{Comparison of OFDM and SC Systems using TDM and FDM Pilots}

In this section, we compare the performance of OFDM and SC systems using TDM and FDM pilots, in terms of pilot overhead, CP overhead, the required power backoff for the HPA and the FER performance. Fig. 5.12 and Fig. 5.13 show the FER performance of a coded OFDM system with FDM pilots using non-iterative (Wiener $2 \times 1 \mathrm{D})$ and iterative channel estimation (ICE), respectively. The results for ICE are obtained using the iterative channel estimator briefly described in Sec. 2.6.4 and proposed in [56]. Note that the case for coded OFDM systems using FDSPT pilots

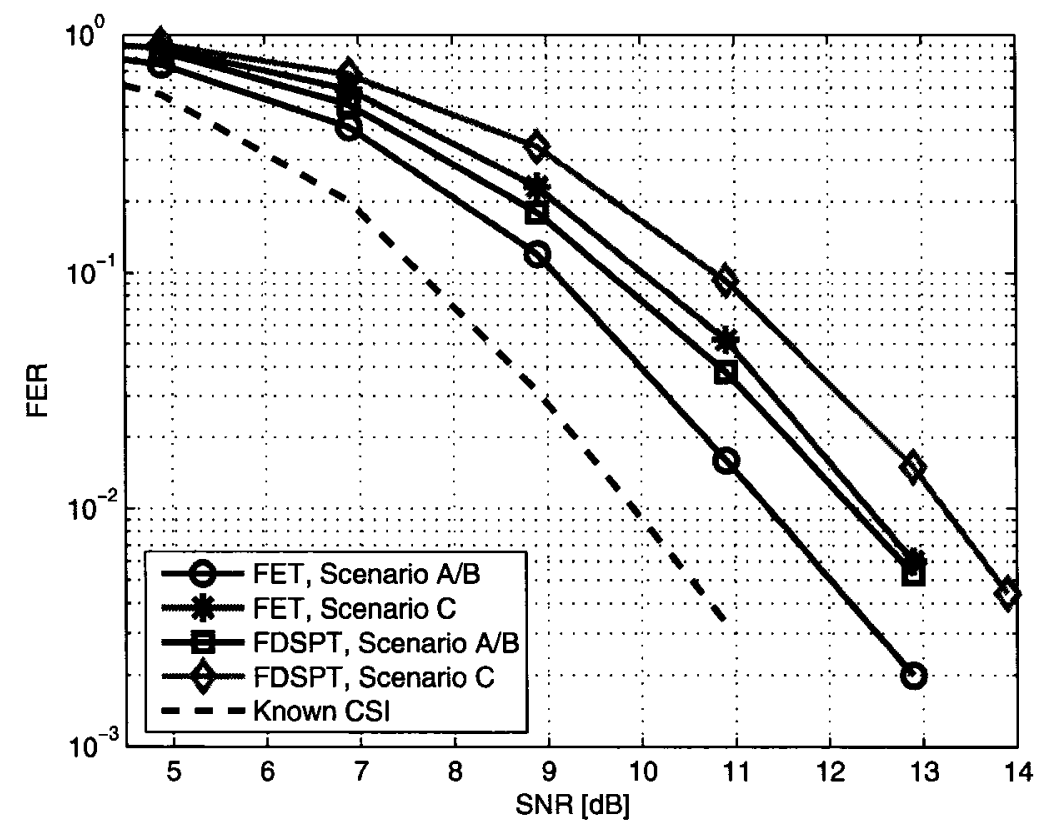

Figure 5.12: FER Performance of FDM Pilots for Coded OFDM Systems using Linear Equalizer and Non-iteratve Channel Estimator (Parameter Set 2-A)

is not considered in [56]. In OFDM systems, since each data symbol is represented by a subcarrier, the removal of the data tones for pilot tones permanently removes the data symbols at the pilot locations. Hence, for an OFDM system, the use of FDSPT pilot must be accompanied by channel coding, which can be used to recover the loss of data symbols (see also Fig. 5.8). This is a case similar to the OFDM systems with 


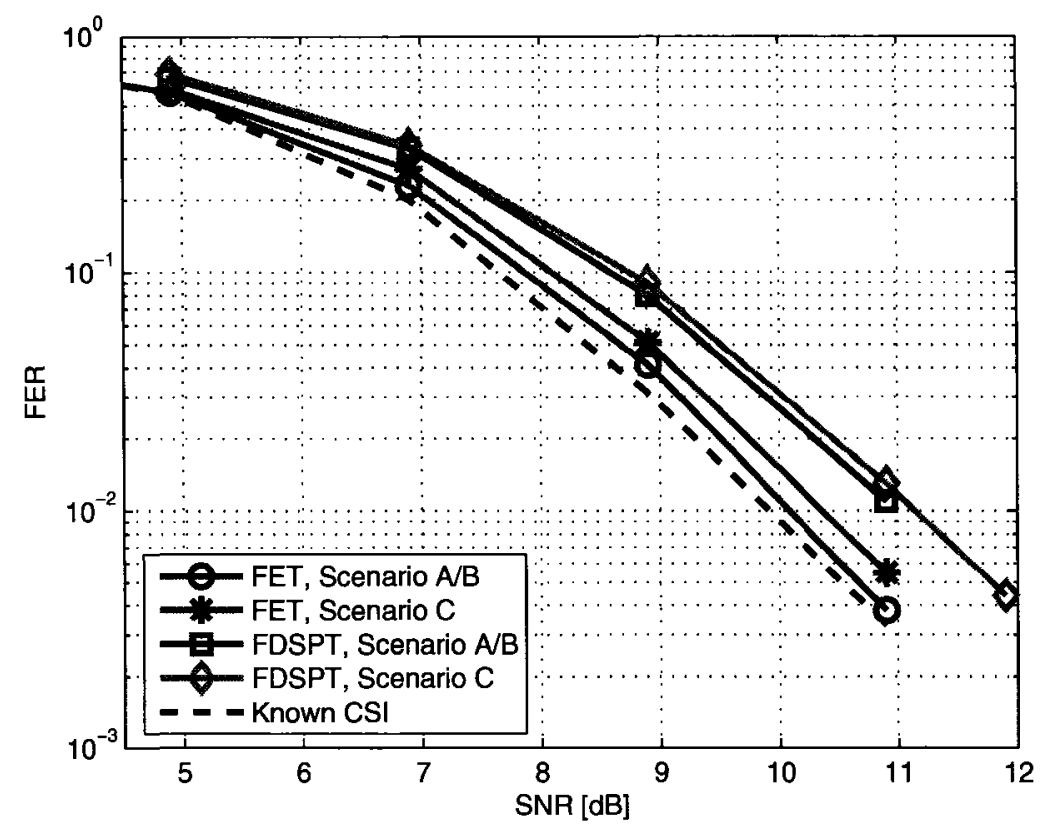

Figure 5.13: FER Performance of FDM Pilots for Coded OFDM Systems using Linear Equalizer and Iterative Channel Estimator (Parameter Set 2-A)

punctured codes, if the FDSPT pilot scheme with $\alpha=0$ is used.

Using the results from Fig. 5.10 to Fig. 5.13, Table 5.2 summarizes the FER performance of the coded OFDM and SC systems, in terms of SNR for a FER of $10^{-2}$, the pilot and CP overhead of non-iterative and iterative channel estimators. Since the performance between Scenario A and B are similar, their performance is shown in the same column. Comparing the performance of Scenario A with the performance of Scenario $\mathrm{C}$ for $\mathrm{W} 2 \times 1 \mathrm{D}$ and IDDCE, the price for lack of the exact channel knowledge is about $1 \mathrm{~dB}$ and $0.4 \mathrm{~dB}$ respectively. Using TDM pilots requires slightly more pilots. Notice that in all cases the cyclic prefix is still the main source of overhead in a block.

As discussed in Sec. 4.2.3, using FDM pilots for SC systems requires slightly higher power backoff of a HPA than using TDM pilots although it is still lower than the required power backoff for OFDM systems. Fig. 5.14 shows the power spectra of the SC signals with different pilot schemes and OFDM signal with FET pilots, where $\rho$ 
Table 5.2: Comparison of OFDM and SC Performance and Overhead for TDM and FDM Pilot Schemes (SNR at FER $=10^{-2}$ is obtained from Fig. 5.10 to Fig. 5.13 and IBSDFE equalizer is used for SC systems)

\begin{tabular}{|c|c|c|c|c|c|c|}
\hline \multirow[t]{4}{*}{ Pilot Schemes } & \multicolumn{4}{|c|}{$\mathrm{SNR}(\mathrm{dB})$ at $\mathrm{FER}=10^{-2}$} & \multirow{4}{*}{$\begin{array}{c}\text { Pilot } \\
\text { overhead }\end{array}$} & \multirow{4}{*}{$\begin{array}{c}\text { Cyclic } \\
\text { prefix } \\
\text { overhead } \\
\left(\frac{\text { total } \mathrm{CP}}{\text { total data }}\right)\end{array}$} \\
\hline & \multicolumn{4}{|c|}{ Scenario } & & \\
\hline & \multicolumn{2}{|c|}{$A$ or $B$} & \multicolumn{2}{|c|}{$\mathrm{C}$} & & \\
\hline & $\mathrm{W} 2 \times 1 \mathrm{D}$ & IDDCE & $\mathrm{W} 2 \times 1 \mathrm{D}$ & IDDCE & & \\
\hline OFDM-Known CSI & 10.0 & 10.0 & 10.0 & 10.0 & $\mathrm{~N} / \mathrm{A}$ & $16.1 \%$ \\
\hline OFDM-FET & 11.4 & 10.1 & 12.4 & 10.4 & $4.4 \%$ & $16.1 \%$ \\
\hline OFDM-FDSPT & 12.3 & 11.0 & 13.2 & 11.2 & $0 \%$ & $16.1 \%$ \\
\hline SC-Known CSI & 9.3 & 9.3 & 9.3 & 9.3 & $\mathrm{~N} / \mathrm{A}$ & $16.1 \%$ \\
\hline SC-TDM & 10.9 & 9.7 & 11.9 & 10.1 & $5.1 \%$ & $18.0 \%$ \\
\hline SC-FET & 10.7 & 9.7 & 12.1 & 10.1 & $4.4 \%$ & $16.1 \%$ \\
\hline SC-FDSPT & 11.7 & 10.5 & 12.9 & 10.9 & $0 \%$ & $16.1 \%$ \\
\hline
\end{tabular}

denotes the required power backoff, 104 pilots are added in the first and the last block $(M=416)$ of a frame $(Q=12)$ and a $C h u$ sequence is used as the pilot sequence. The value of $\rho$ in each case was determined so as to accommodate the transmitted spectrum emerging from a power amplifier modelled by a Rapp model with $p_{o}=2$ [113] (or see (7.13)), satisfying a typical spectral mask. Table 5.3 summarizes the required power backoff in $\mathrm{dB}$ for SC and OFDM systems. The SC signal with TDM

Table 5.3: Comparison of Required Power Backoffs for OFDM and SC Systems

\begin{tabular}{|c|c|c|c|}
\hline Pilot Schemes & $\begin{array}{c}\text { Power back-off } \\
\text { to satisfy } \\
\text { spectral mask }\end{array}$ & $\begin{array}{c}\text { Additional back-off } \\
\text { for pilot blocks }\end{array}$ & $\begin{array}{c}\text { Average data SNR } \\
\text { penalty due to increased } \\
\text { back-off on pilot blocks }\end{array}$ \\
\hline OFDM-FET & 10 & 0 & 0 \\
\hline SC-TDM & 8 & 0 & 0 \\
\hline SC-FET & 8 & 1 & 0.2 \\
\hline SC-FDSPT & 8 & 1 & 0.2 \\
\hline
\end{tabular}

pilots requires the least power backoff $(8 \mathrm{~dB})$, while OFDM requires the most $(10 \mathrm{~dB})$ among different pilot schemes. The SC signals with FDM pilots requires additional 


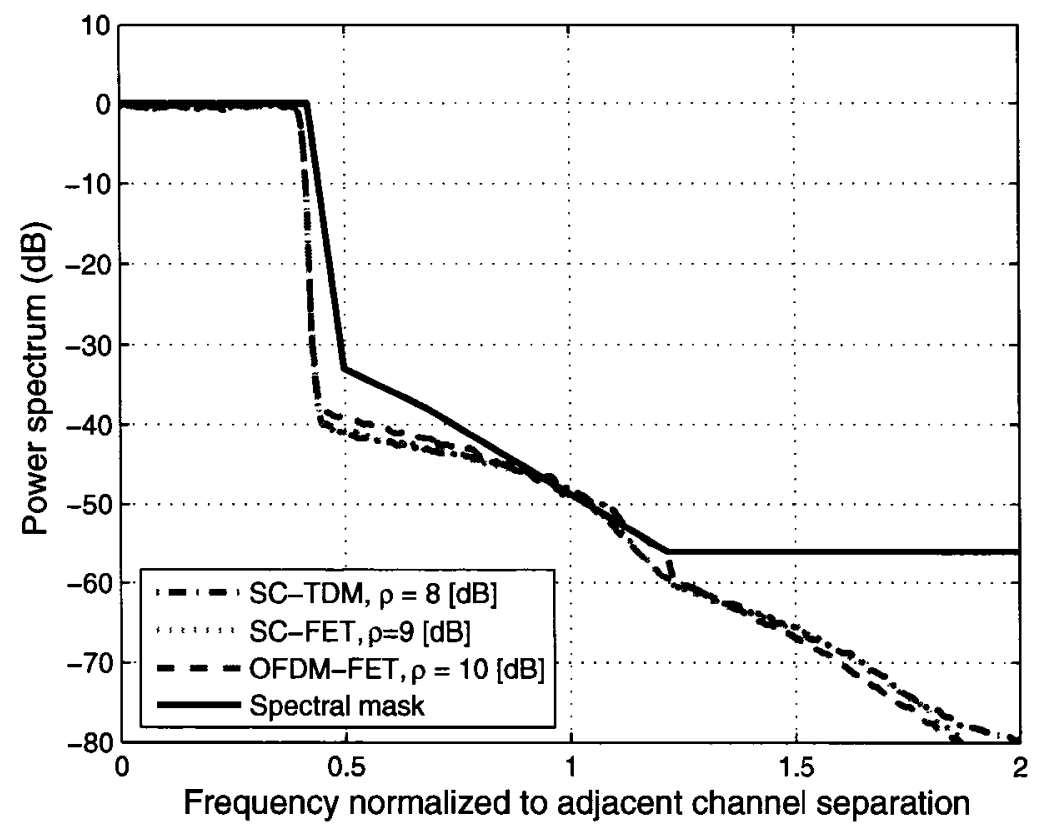

Figure 5.14: Power Spectra of Signal Emerging from a Rapp Model $p_{o}=2$ Power Amplifier, with Back-offs Required to Satisfy a Spectral Mask, $\rho=$ Power Back-off

$1 \mathrm{~dB}$ power backoff for the blocks with pilots, resulting in a reduction of data power and received SNR if the pilot power is to be fixed for not compromising the channel estimation performance. Since not all the blocks are with pilots and the channel coding and interleaving are carried over a frame of 12 blocks, the effect is small, as seen in the last column of Table 5.3. For this particular example, a serial modem with TDM or FDM pilots could be designed with a power amplifier with about $2 \mathrm{~dB}$ lower maximum power rating than that of a corresponding OFDM modem.

Qualitatively similar results are obtained for other values of the Rapp parameter $p_{o}$, and for different numbers and configurations of pilots. $p_{o}=2$ corresponds to a relatively low quality power amplifier that has a significant AM/AM nonlinearity even for input voltages below the saturation level. For $p_{o}=10$ power back-offs can be achieved of $8.5 \mathrm{~dB}$ and $6 \mathrm{~dB}$ for OFDM and serial modulation respectively. Further PAPR and back-off reduction can be achieved for serial modulation if square root raised cosine filtering with excess bandwidth is applied. 


\subsection{Summary}

Channel estimation techniques for OFDM systems with PACE can be used, with some modifications, for SC systems with FDM pilots. The low complexity FFT-LLSLF channel estimator gives the worst MSE performance, while the frequency domain IDDCE with optimum MMSE estimation and decoder provides the best MSE performance among the proposed channel estimators, with higher complexity. The low complexity FR algorithm with a threshold is used to replace the optimum MMSE estimator, with a slight performance degradation. The cascaded $2 \times 1 \mathrm{D}$ Wiener channel estimator outperforms both hybrid channel estimators. The FFT-Wiener estimator produces similar MSE performance as that of $2 \times 1 \mathrm{D}$ Wiener channel estimator for low SNRs $(<10 \mathrm{~dB})$. At low SNRs $(<10 \mathrm{~dB})$, the MSE performance of the frequency domain IDDCE depends on the reliability of the tentative decisions. A simple hard decision re-encoding can be used to improve the reliability of the tentative decisions at the expense of slightly higher complexity.

Using the non-iterative $\mathrm{W} 2 \times 1 \mathrm{D}$ Wiener estimator, the FER performance degradation for full bandwidth system is about $1.4 \mathrm{~dB}$ and $2.4 \mathrm{~dB}$ for FET and FDSPT respectively. An additional $1 \mathrm{~dB}$ of $\mathrm{SNR}$ is needed if exact knowledge of the maximum delay spread and the speed of the user terminal is not known and the worse case channel knowledge is assumed for generating the Wiener filter taps. Using the proposed low complexity IDDCE with the FR algorithm, the FER performance for coded SC systems using FET pilots and FDSPT pilots with iterative interference cancellation was found to be about $0.2 \mathrm{~dB}$ and about $0.5 \mathrm{~dB}$ away from the FER performance with known CFR at FER $=10^{-2}$, respectively. FDSPT pilots can also be used for OFDM systems with channel coding. It was found that an extra $1 \mathrm{~dB}$ of SNR is required at FER $10^{-2}$, compared with that using the conventional FET pilots for OFDM systems. Note however that FDSPT pilots require no pilot overhead. Taking into account the required power backoff of a HPA, a serial modem with TDM or FDM pilots could be 
designed with a power amplifier with about $2 \mathrm{~dB}$ lower maximum power rating than that of a corresponding OFDM modem. 


\section{Chapter 6}

\section{Channel Estimation using FDM Pilots for Chunk and Sub-chunk Size Systems}

This chapter deals with channel estimation for chunk and sub-chunk size systems with FDM pilots. We consider non-iterative (Wiener $2 \times 1 \mathrm{D}$ ) and iterative (IDDCE) channel estimation for sub-chunk-based (IFDMA ${ }^{1}$ and block-IFDMA (B-IFDMA)) systems using the proposed FDM pilot arrangements. Similar to the full bandwidth case, the effects of mis-matched channel knowledge on the performance is included. We also consider channel estimation over multiple half or full duplex FDD (frequency division duplex) frames, which is shown to outperform channel estimation over one frame. The effectiveness of multi-frame channel estimation depends on the pilot spacings in time, which is upper bounded by the sampling theorem. The frame error rate (FER) performance loss due to imperfect channel estimation is shown to be more for B-IFDMA than for IFDMA, full chunk size and full bandwidth systems.

\subsection{Introduction}

To facilitate adaptive transmission, the transmitted data is arranged in a basic timefrequency unit, termed a chunk, which contains a small number of consecutive subcar-

\footnotetext{
${ }^{1}$ In Appendix B, we compare the performance between IFDMA and conventional direct sequence CDMA (DS-CDMA) systems with and without carrier frequency offsets and Wiener phase noise. Note that this topic is included in the appendix for the coherence of the thesis.
} 
riers for some consecutive OFDM symbols [67], as shown in Fig. 6.1. Each user can be assigned multiple chunks within the same signal bandwidth for better frequency diversity gain. Interleaved frequency domain multiple access (IFDMA) [78], which has the same PAPR property as a SC signal, can be considered as a case of a sub-chunk size system, where each user is assigned a single subcarrier in each of a set of equallyspaced chunks. When intra-chunk sleep mode ${ }^{2}$ is enabled in a regular chunk based system, it is termed block-IFDMA (B-IFDMA) [96,97]. Fig. 6.3 and 6.4 illustrate subcarrier placements, including pilots, for IFDMA and B-IFDMA signals. These sub-chunk signals occupy small blocks, smaller than chunks, and equally-spaced, in multiples of the chunk size, in frequency and time. As pointed out in [97], they are useful for non-frequency-adaptive transmission modes, assuming the chunks or blocks are far enough apart in frequency to fade independently.

For both chunk and sub-chunk (IFDMA and B-IFDMA) size systems, the separation between blocks is chosen to be larger than the coherence bandwidth so that higher frequency diversity gain can be obtained. This separation requirement however increases the overall number of pilots being added within a frame (defined as $N_{B}$ consecutive OFDM symbols) for satisfactory channel estimation performance, resulting from the fact that inter-chunk interpolation is not applicable. In [98], various practical channel interpolation techniques in frequency for chunk-based OFDMA systems are studied, while in [99], chunk-based channel estimation in time and frequency for uplink OFDM system is investigated using iterative channel estimation (ICE) over one frame. Both [98] and [99] consider regular chunk size OFDM system (8 subcarriers per OFDM symbols per chunk) with multiple pilots.

In this chapter, we consider non-iterative and iterative pilot-assisted channel estimation using cascaded $2 \times 1$ dimensional $(2 \times 1 \mathrm{D})$ Wiener filtering [81] for IFDMA and B-IFDMA systems. In particular, we consider channel estimation for chunk and

\footnotetext{
${ }^{2}$ In intra-chunk sleep mode, the transmission is switched off for some OFDM symbols for power savings in the user terminal $[96,97]$.
} 
sub-chunk size systems ${ }^{3}$ using FDM pilots. In [95], consecutive previously received OFDM symbols are used to smooth the frequency channel estimates for the current OFDM symbol to improve the performance of the channel estimation. We also consider channel estimation (smoothing and interpolation) over multiple half duplex frames (in which half of the frame is used for transmission and half for reception) to improve the performance of chunk- and sub-chunk-based channel estimation.

\subsection{Description of Chunk and Sub-chunk Size Sys- tems}

\subsubsection{Chunk Size Systems}

A chunk [67] is defined as the smallest unit of time-frequency resource allocated to the users in the system, as shown in Fig. 6.1. A chunk consists of $N_{f}$ consecutive

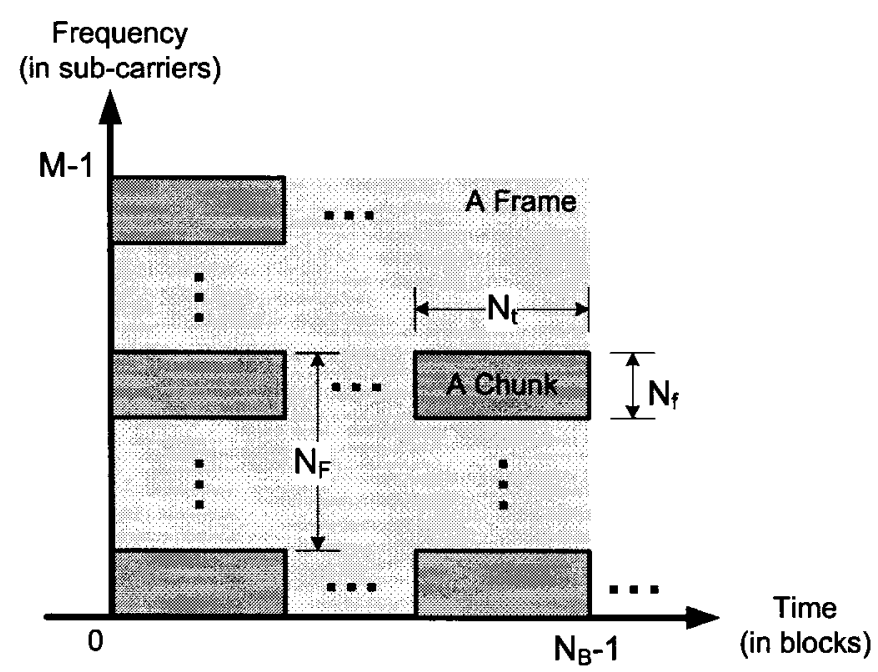

Figure 6.1: Resource Allocations of a Chunk Size System for a Frame

subcarriers for the consecutive $N_{t}$ blocks. In general, the values of $N_{f}$ and $N_{t}$ are chosen such that there is minimal frequency- and time-selectivity within a chunk. In [67], $N_{t}=12$ and $N_{f}=8(12 \times 8=96$ subcarriers $)$ are chosen for a chunk in the wide area scenario, which will be considered as a regular chunk size in this thesis. Recall

\footnotetext{
${ }^{3}$ Both chunk and sub-chunk size systems can have DFT-precoded OFDM or OFDM versions. The OFDM version of B-IFDMA is called B-EFDMA [97].
} 
that we define a frame as an $N_{B} \times M$ time-frequency plane in Sec. 2.6.1. If $\left\lfloor\frac{N_{B}}{N_{t}}\right\rfloor \geq 2$ or $\left\lfloor\frac{M}{N_{f}}\right\rfloor \geq 2$, each user can have multiple chunks within a frame. It is obvious that the smallest chunk is one subcarrier, i.e. $N_{t}=1$ and $N_{f}=1$. To generate SC signals with FDM pilots for chunk size systems, we can modify the vector of multiplexed data and pilot tones $\mathbf{X}$, denoted as $\mathbf{X}_{S}$ for FDSPT pilots (see (4.1)) and $\mathbf{X}_{E}$ for FET pilots (see (4.7)) in the case of full bandwidth systems, and use Fig. 2.4 with the modified vector $\mathbf{X}$. The vector of multiplexed data and pilot tone for SC signals with FET pilots for chunk size system is given as

$$
\begin{aligned}
& \mathbf{X}_{e}=[\underbrace{P_{0}, A_{0}, \ldots, A_{K-2}, P_{1}, \ldots, A_{N_{f}-3}}_{\text {one block }}, \mathbf{0}_{N_{F}-N_{f}}, P_{2}, A_{N_{f}-2}, \ldots, \\
& \left.P_{N-2}, A_{\left(N_{b l k}-1\right)\left(N_{f}-2\right)}, \ldots, P_{N-1}, \ldots, A_{N_{b l k}\left(N_{f}-2\right)-1}, \mathbf{0}_{N_{F}-N_{f}}\right]^{T},
\end{aligned}
$$

where $K$ denotes the pilot spacing, $\mathbf{0}_{N_{F}}$ is a zero row vector of size $N_{F}, N_{F}$ is the subcarrier separation between chunks, $N$ is the total number of pilots for an OFDM symbol, $N_{f}$ is the number of subcarriers per OFDM symbol per block, $N_{b l k}$ is the number of blocks of subcarriers per OFDM symbol, $P_{\ell}$ is the $\ell$ th pilot tone and $A_{\ell}$ is the $\ell$ th data tone. Comparing (6.1) with (4.7), it is apparent that the zero vector $\mathbf{0}_{N_{F}-N_{f}}$ is inserted in between blocks of subcarriers, while for full bandwidth systems, all the subcarriers are either data or pilot tones. Notice that in total $N_{b l k} \cdot\left(N_{f}-2\right)$ data symbols per OFDM symbol (with pilots) can be transmitted in this OFDM symbol. Similarly, for FDSPT pilots $(\alpha=0), \mathbf{X}$ can be obtained as

$$
\begin{aligned}
\mathbf{X}_{s}=[\underbrace{P_{0}, A_{1}, \ldots, A_{K-1}, P_{1}, \ldots, A_{N_{f}-1}}_{\text {one block }}, \mathbf{0}_{N_{F}-N_{f}}, P_{2}, A_{N_{f}}, \ldots, \\
\left.P_{N-2}, A_{\left(N_{b l k}-1\right) N_{f}}, \ldots, P_{N-1}, \ldots, A_{N_{b l k} N_{f}-1}, \mathbf{0}_{N_{F}-N_{f}}\right]^{T} .
\end{aligned}
$$

Note that in this case $N_{b l k} \cdot N_{f}$ data symbols per OFDM symbol (with pilots) can be transmitted. As an example, consider the chunk size system with $N_{t}=12$ and $N_{f}=8$. For a frame with $M=416$ and $N_{B}=12$ (or Parameter Set 2-A), we have $\frac{M}{8}=52$ chunks per frame. Fig. 6.2 shows an example of the chunk resource 
allocations for 5 users with frame size 416 sub-carriers $\times 12$ blocks, and with two FDM pilot symbols at the first and the last block of each chunk. Starting from the

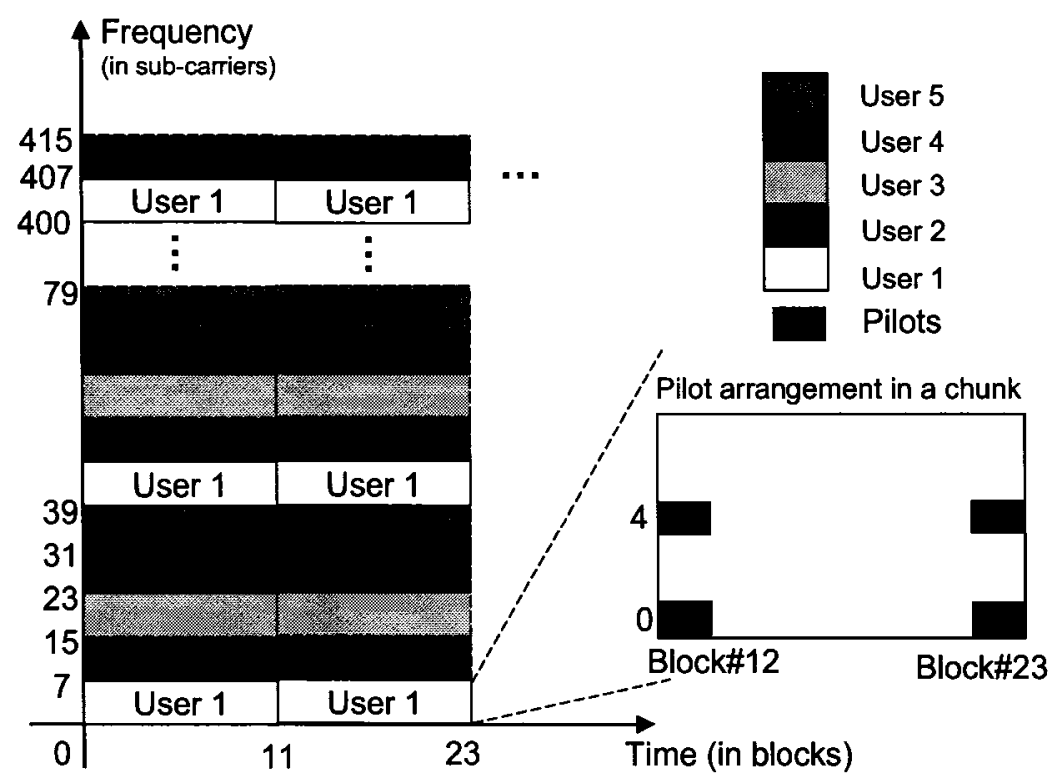

Figure 6.2: Allocation of Chunks for Five Active Users

0th subcarrier, the chunks are allocated to each user in a round-robin manner such that the first two users are assigned 11 chunks, while the last three users are assigned 10 chunks. Note that blocks of subcarriers are separated at a sufficient distance so as to gain full frequency diversity. We assume the same allocation scheme is used for each frame. Note that some of the simulation results, such as those in Fig. 6.12 and Fig. 6.13, are based on the example shown in Fig. 6.2.

\subsubsection{Sub-chunk Systems}

The generation of SC signals for sub-chunk size systems with FDM pilots is similar to that for chunk size systems. We only need a different vector of multiplexed data and pilot tones X. For IFDMA systems with FET pilots, since each block of subcarriers has only one subcarrier, $\mathbf{X}$ contains only the pilot tones, given as

$$
\mathbf{X}_{e}^{I}=\left[P_{0}, \mathbf{0}_{N_{F}-1}, P_{1}, \mathbf{0}_{N_{F}-1}, \ldots, P_{N-1}, \mathbf{0}_{N_{F}-1}\right]^{T}
$$


In this case, $N_{b l k}=N$ and no data symbols can be transmitted in this OFDM symbol. It is obvious from (6.3) that interpolation is impossible (resulting in higher pilot overhead) if the coherence bandwidth exceeds the separation between blocks of subcarriers, i.e.

$$
B_{c}<N_{F} \cdot \Delta F
$$

where $N_{F}$ is the separation in subcarriers, $\Delta F$ is the sub-carrier spacing in $\mathrm{Hz}$ and $B_{c}$ denotes the coherence bandwidth, which can be estimated as [34]

$$
B_{c} \approx \frac{1}{5 \sigma_{\tau}}
$$

where $\sigma_{\tau}$ is the root mean square (rms) delay spread of the channel. For IFDMA systems with FDSPT with $\alpha \neq 0$, the vector of superimposed pilot and data tones is given as

$$
\mathbf{X}_{s}^{I}=\left[\alpha A_{0}+\beta P_{0}, \mathbf{0}_{N_{F}-1}, \alpha A_{1}+\beta P_{1}, \mathbf{0}_{N_{F}-1}, \ldots, \alpha A_{N-1}+\beta P_{N-1}, \mathbf{0}_{N_{F}-1}\right]^{T}
$$

where $\alpha \neq 0$ and $\beta$ are the scaling factor for data and pilot tones, respectively. In this case, $N$ (or $N_{b l k}$ ) data symbols can be transmitted. However, since $\alpha \neq 0$, a more complex channel estimator is necessary to cancel the inference resulting from the data tones, such as time averaging and joint equalization and channel estimation [15].

For B-IFDMA systems with FET pilots, the vector of multiplexed data and pilot tones is given as

$$
\begin{aligned}
\mathbf{X}_{e}^{B}= & {[\underbrace{P_{0}, A_{0}, \ldots, A_{K-2}, P_{1}, \ldots, A_{N_{c}-3}}_{\text {one block }}, \mathbf{0}_{N_{F}-N_{c}}, P_{2}, A_{N_{c}-2}, \ldots,} \\
P_{N-2}, A_{\left(N_{b l k}-1\right)\left(N_{c}-2\right)}, \ldots, P_{N-1}, \ldots, A_{N_{b l k}\left(N_{c}-2\right)-1}, \mathbf{0}_{N_{F}-N_{c}} & ]^{T},
\end{aligned}
$$

where $0_{N_{F}-N_{c}}$ is a zero row vector of size $N_{F}-N_{c}, N_{F}$ is the subcarrier separation between chunks, $N$ is the total number of pilots for an OFDM symbol, $N_{c}<N_{f}$ is the number of subcarriers per OFDM symbol per chunk, $P_{\ell}$ is the $\ell$ th pilot tone and $A_{\ell}$ is the $\ell$ th data tone. Notice that if $N_{c}=N_{f},(6.7)$ is equivalent to (6.1). Replacing 
$N_{f}$ in (6.2) with $N_{c}$, we have the vector $\mathrm{X}$ for B-IFDMA system with FDSPT pilots $(\alpha=0)$, given as

$$
\begin{aligned}
\mathbf{X}_{s}^{B}=[\underbrace{P_{0}, A_{1}, \ldots, A_{K-1}, P_{1}, \ldots, A_{N_{c}-1}}_{\text {one block }}, \mathbf{0}_{N_{F}-N_{c}}, P_{2}, A_{N_{f}}, \ldots, \\
\left.P_{N-2}, A_{\left(N_{b l k}-1\right) N_{f}}, \ldots, P_{N-1}, \ldots, A_{N_{b l k} N_{f}-1}, \mathbf{0}_{N_{F}-N_{c}}\right]^{T} .
\end{aligned}
$$

\subsection{Chunk and Sub-chunk Size Channel Estima- tion over One Frame}

For chunk size and B-IFDMA systems, more than one pilot can be placed for each block of subcarrier, while for IFDMA systems, no more than one pilot can be used for each single-subcarrier block. Channel estimation techniques using FDM pilots for full bandwidth systems, discussed in Chapter 5, can be applied for the chunk size systems. The difference is that we only need to estimate the channel frequency response for the frequencies assigned to the desired user. We consider low complexity LLSLF in both time- and frequency- direction as usually the time and frequency selectivity of the channel within a chunk are linear. We also apply the cascaded $2 \times 1 \mathrm{D}$ Wiener filtering and the proposed IDDCE channel estimation techniques for chunk and sub-chunk size systems.

\subsubsection{Pilot Arrangements for Sub-chunk Size Systems}

Fig. 6.3 shows an example of a pilot arrangement for IFDMA systems, where $N_{F}=32$ and the first and the last OFDM symbols of a frame of $N_{B}=12$ OFDM symbols are pilots. Pilot-carrying subcarriers are spaced by $N_{F}=32$ subcarriers, which is assumed to be enough to yield substantial frequency diversity in the non-adaptive transmission mode. The pilot overhead is $2 / N_{B}=1 / 6$ and the number of useful subcarriers is $N_{F} \times N_{c} \times N_{B}=384$. Note that for IFDMA $N_{c}=1$ and the number of active OFDM symbols, denoted as $Q$, for channel estimation is $Q=N_{B}=12$.

Fig. 6.4 shows three possible pilot arrangements for B-IFDMA systems with 


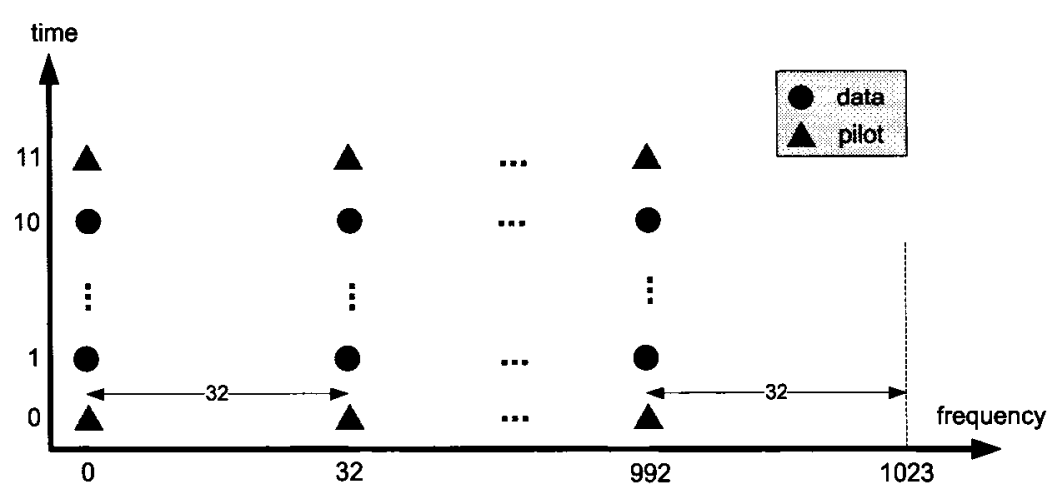

Figure 6.3: F1T2 Pilot Arrangement for IFDMA Systems

$N_{c}=4, N_{F}=32, N_{b l k}=32$ and $Q=3$, where $Q$ is the number of active OFDM symbols (or the window size) for channel estimation. Note that since $N_{f}=2 N_{c}$, we also call the regular chunk size systems $2 B$-IFDMA systems. The total number of useful subcarriers is $N_{b l k} \times N_{c} \times Q=384$, which is the same as that of the IFDMA system described in Fig. 6.3. Also notice that for this B-IFDMA system, there are only 3 OFDM symbols that are transmitting in a frame, while the remaining 9 OFDM symbols are in sleep mode to save energy [97]. The designation "FxTy" means that pilot-carrying OFDM symbols contain $x$ pilots per chunk per OFDM symbol, and that $y$ OFDM symbols per chunk contain pilots. F1T1 incurs a pilot overhead of $1 / 12$ and F1T2 incurs a pilot overhead of 2/12. In these arrangements, the same inter-block spacing of 32 subcarriers is used, assuring good frequency diversity between blocks. Note that when a single pilot is used in either time or frequency, the channel estimates in time or frequency are assumed to be constant.

\subsubsection{Non-iterative Channel Estimation}

Assuming that the chunk size is selected so that the time- and frequency- selectivity are linear, for low complexity a LLSLF interpolator, requiring no channel statistics, can be used to estimate the channel frequency response for a chunk. The LLSLF requires at least four pilot symbols within a chunk, as shown in Fig. 6.2. The application of cascaded $2 \times D$ FIR Wiener filtering for chunk size and B-IFDMA 


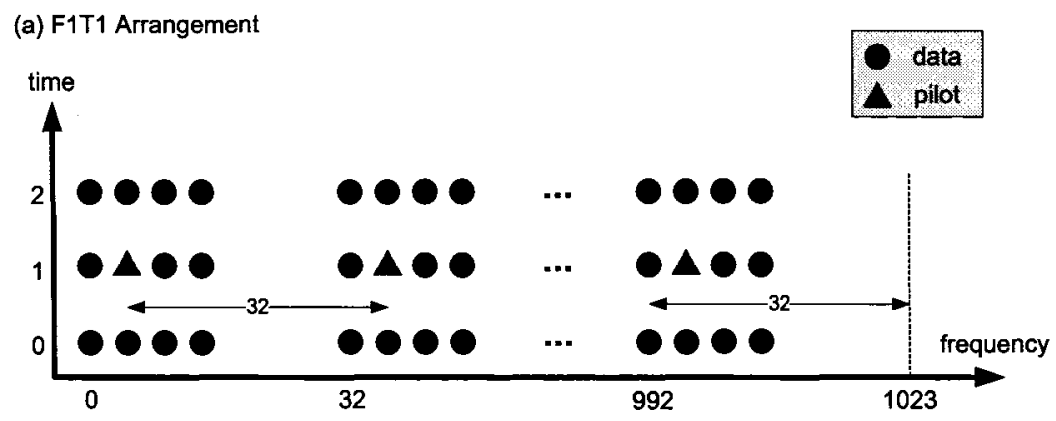

(b) F2T1 Arrangement
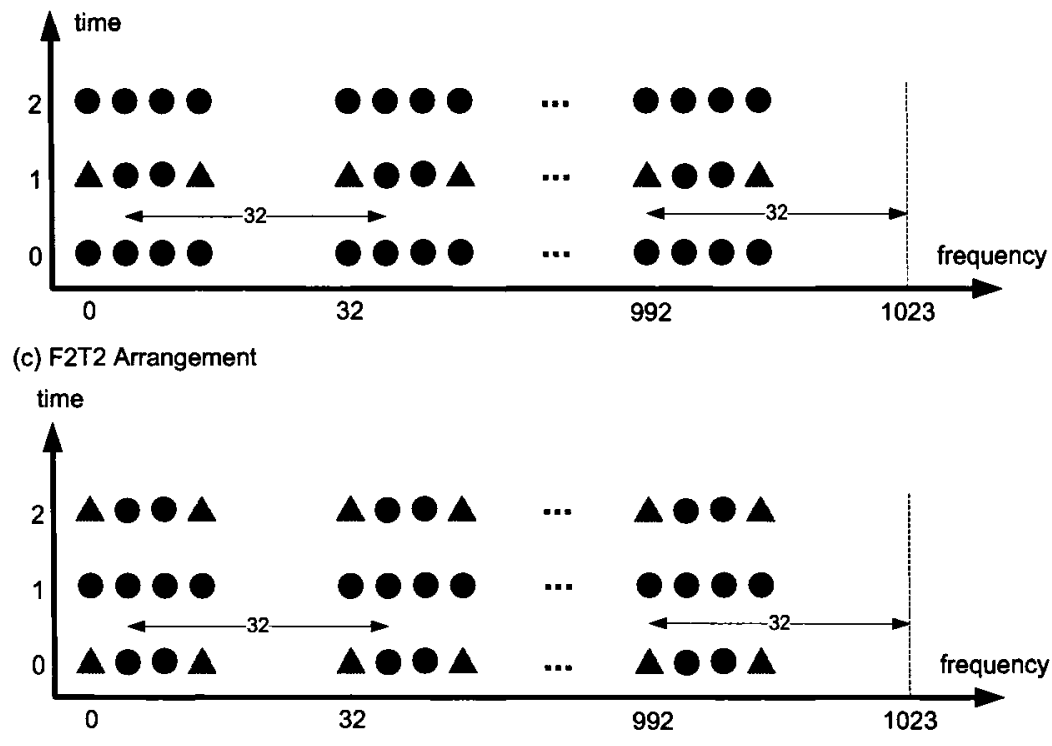

Figure 6.4: Pilot Arrangements for B-IFDMA Systems 
systems is similar to that for full bandwidth systems. The number of filter taps is limited by the number of pilots (usually small) used within a chunk or sub-chunk. In our specific example (Fig. 6.2), the number of filter taps in both time- and frequencydirection is two, i.e. $I_{f}=I_{t}=2$. The small number of filter taps limits the MSE performance of the Wiener filtering. Note that when the frequency distances among blocks of subcarriers are close enough, the pilots from multiple blocks of subcarriers can be also used to interpolate the channel frequency response. However, less frequency diversity would be expected in this case, meaning that there exists a trade-off between frequency diversity and pilot overhead.

\subsubsection{Iterative Decision-Directed Channel Estimation}

When iterative channel estimators are used, more pilots can be used for channel estimation after the initial estimation. In our five user example, after the initial estimation, the number of filter taps in frequency direction is $I_{f}=8$ and in the time direction $I_{t}=Q=12$. This will enhance the MSE performance of the chunk size channel estimators, as shown in Section 6.5. In case of B-IFDMA, we have $I_{f}=4$ and in the time direction $I_{t}=Q=12$. For IFDMA, we have $I_{f}=1$, which means no frequency diversity and implies worse performance, and $I_{t}=12$.

\subsection{Channel Estimation for Chunk and Sub-chunk Size Systems over Multiple Frames}

Interpolation and smoothing of channel estimates over more than one frame has the potential to improve channel estimation performance, given that a certain amount of correlation of the channel in time exists ${ }^{4}$. Fig. 6.5 shows pilot arrangements for smoothing over multiple half-duplex frames ${ }^{5}$, where 'FB' stands for full bandwidth. $Q$ is the number of OFDM symbols over which smoothing is performed. For example

\footnotetext{
${ }^{4}$ The higher the terminal velocity, the less time correlation between OFDM symbols exists, and the less effective will be multi-frame estimation.

${ }^{5}$ For each half-duplex frame (consisting of 24 OFDM symbols), half of the frame is used for transmission and half for reception.
} 
$Q=48$ for IFDMA indicates smoothing over 4 successive half-duplex frames, and $Q=6$ for B-IFDMA indicates smoothing over 2 successive half-duplex frames. Note that a sleep mode of 9 OFDM symbols is included for the B-IFDMA system of Fig. 6.4. It is obvious smoothing over multiple frames improves the overall channel

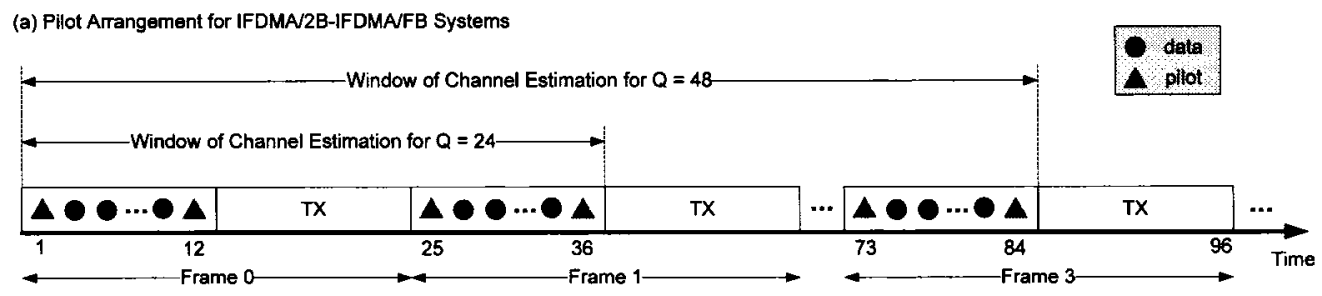

(b) Pilot Artangement for B-IFDMA Systems

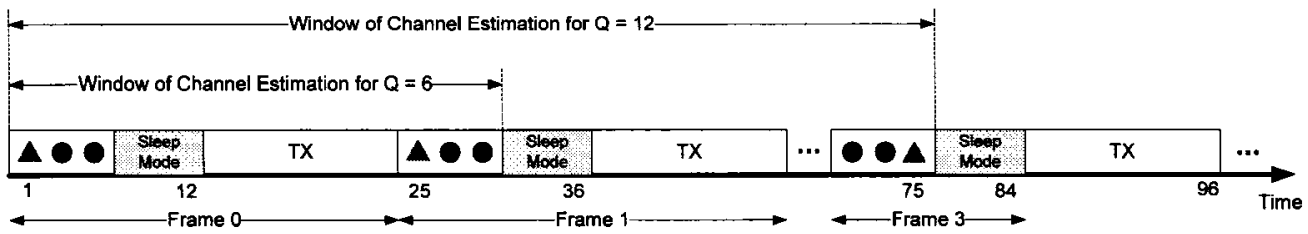

Figure 6.5: Pilot Arrangement for Multiple Half Duplex Frames

performance of channel estimates for all the frames. More pseudo pilots (and thus higher filter order in time) are available for channel estimation if we consider the halfduplex frames within the estimation window as a super frame with $Q$ consecutive OFDM symbols. For example, in Fig. 6.5 (a) $Q$ can be either 24 or 48 OFDM symbols, while in Fig. 6.5 (b) $Q$ can be either 6 or 12 OFDM symbols. The frequency channel estimates are obtained for only the active OFDM symbols. In other words, it is not necessary to obtain the frequency channel estimates for the transmission (TX) period or sleep mode of B-IFDMA. Moreover, the pilots for the super frame are not uniformly distributed any more. For example, in Fig. 6.5 (a) for $Q=48$, the pilots are located at $\mathbf{k}_{48} \in\{1,12,25,36,49,60,73,84\}$. In this case, the order of the filter $D$ is 8 . Although the pilot locations $\mathbf{k}_{48}$ are slightly non-uniformly distributed, the interpolation is still valid as long as their sampling instants deviate less than $(1 / \pi) \ln 2 \approx 0.22$ from its corresponding Nyquist (or oversampling) instant [100]. For the pilot arrangement $\mathbf{k}_{48}$, the maximum deviation is $1 / 12 \approx 0.083$, assuming the 
following uniform pilot arrangement $\{1,13,25,37,49,61,73,85\}$.

The interpolation of the channel estimates at the $\ell$ th subcarrier in active OFDM symbols can be obtained using (2.58) with $\mathbf{k}_{48}$ as the pilot locations and with $k \in$ $\{1,2, \ldots, 12,25,26, \ldots, 36,49,50, \ldots, 60,73,74, \ldots, 84\}$ as the time index. Similarly for B-IFDMA in Fig. 6.5 (b), $k \in\{1,2,3,25,26,27,50,51,52,73,74,75\}$, in which the pilots can be located. The pilots are assumed to be located at $\mathbf{k}_{12}=\{1,25,50,75\}$, as shown in Fig. 6.5 (b). In this case, the deviation of sampling instants (from the uniform sampling instants) is $1 / 24 \approx 0.04$, assuming the following uniform pilot arrangement $\{1,26,51,76\}$. The effectiveness of multi-frame channel estimation depends on the speed (or the maximum Doppler frequency) of the vehicle. The uniform pilot spacing in time, denoted as $D_{t}$, is upper bounded by the sampling theorem, given in the right side of (2.47).

\subsection{Performance Evaluation}

Channel realizations for 2000 frames are used to obtain the averaged MSE performance, while either 10000 frames of channel realizations or a threshold of 100000 errors are used for FER performance. Channel coding is used over a frame $\left(N_{B}=12\right)$ of 12 OFDM symbols for chunk-size, IFDMA and full bandwidth systems, while for B-IFDMA systems with sleep mode, the coding is performed over 3 OFDM symbols. For multi-frame channel estimation, coding is still performed over one frame (12 or 3 OFDM symbols) such that the effect of codeword length on FER performance can be neglected.

\subsubsection{Averaged MSE}

\section{Chunk Size Systems}

Fig. 6.6 shows the averaged MSE of different channel estimators for the five user chunk size system (Fig. 6.2) using parameters Set 2-A. The MSE performance using W2 $\times 1$ D Wiener filter with all Chu pilot symbols are included for comparison. The 


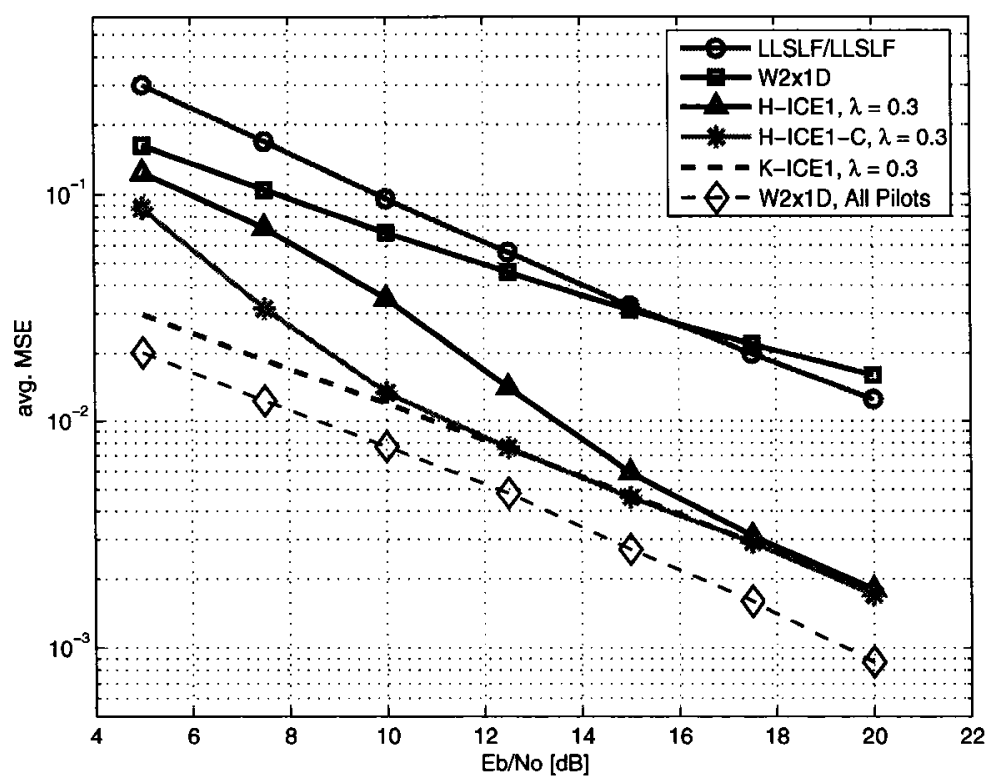

Figure 6.6: MSE Performance of Different Channel Estimators for Chunk Size Systems using Parameters Set 2 and the 5 User Chunk System Shown in Fig. 6.2

time-averaged MSE is calculated over all chunks or sub-chunks within a frame, given as

$$
M S E=\frac{1}{N_{t} N_{f} N_{b l k}} \sum_{n=0}^{N_{b l k}-1} \sum_{k \in \mathbf{K}_{n}} \sum_{\ell \in \mathbf{L}_{n}}\left|\tilde{H}_{k, \ell}^{(n)}-H_{k, \ell}^{(n)}\right|
$$

where $N_{t}$ is the number of active OFDM symbols per frame, $N_{f}$ is the number of subcarriers per block, $N_{b l k}$ is the number of blocks of subcarriers per OFDM symbol, $\mathbf{L}_{n}$ is the set of frequency indexes for the $n$th chunk and $\mathbf{K}_{n}$ is the set of time indexes for the $n$th chunk. Note that for IFDMA, $N_{f}=1$ and $N_{f}=N_{c}$ for B-IFDMA. The cascaded $2 \times 1$ D Wiener filtering outperforms the LLSLF interpolator for $E_{b} / N o \leq 15$ $\mathrm{dB}$. For this specific example, when $E_{b} / N_{o}>15 \mathrm{~dB}$, the LLSLF/LLSLF interpolator gives better MSE performance. The IDDCE using the frequency replacement (FR) algorithm without hard decision re-encoding outperforms the non-iterative channel estimators, especially in the high $E_{b} / N_{o}$ region where more reliable decisions can be used. The MSE performance of the IDDCE using FR algorithm with known symbols is also included for reference. To improve the decision errors at the low $E_{b} / N_{o}$ region, 
the hard decision re-encoding of the estimated data bits can be used.

Comparing the optimum MSE performance of $2 \times 1 \mathrm{D}$ Wiener channel estimation with all the symbols in a chunk being $\mathrm{Chu}$ pilot symbols, using frequency domain IDDCE requires about $2 \mathrm{~dB}$ more of SNR to achieve the same MSE performance. The difference is due to fact that fewer pilots are available for the initial channel estimation and that $\mathrm{Chu}$ pilot symbols have constant magnitude response, which avoids the issue of noise enhancement. Note however that the pilot overhead is $100 \%$ when all pilots are transmitted within a chunk. The MSE performance of the channel estimation for full bandwidth systems is in general better than that of the chunk size systems as there are more pilots for the full bandwidth systems.

\section{Sub-chunk Size Systems}

We next evaluate the averaged MSE of the non-iterative and iterative channel estimation for IFDMA for estimation over one $(Q=12)$, two $(Q=24)$ and four $(Q=48)$ frames, for the half and full duplex cases, as shown in Fig. 6.7 and 6.8. If full duplex FDD (frequency division duplex) is used, the transmission periods (labeled as "TX"), shown without pilots in Fig. 6.5, are eliminated, resulting in a possible denser pilot arrangement. The figure legends indicate which OFDM symbols contain pilots. For example "[ $\left.\begin{array}{llll}1 & 8 & 16 & 24\end{array}\right]$ " in the full duplex FDD case means that there are two successive frames (therefore 24 adjacent OFDM symbols) with pilots in the 1st, 8th, 16th and

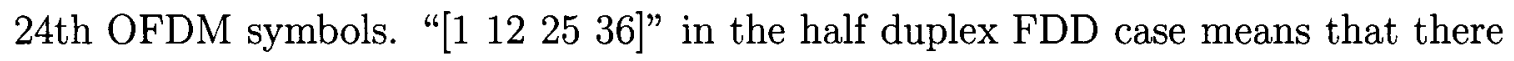
is a $12-\mathrm{OFDM}$ symbol gap between two received frames, so pilots occur in the 1st and 12th OFDM symbol of the first received frame, and also in the 1st and 12th OFDM symbol of the second received frame. This shows clearly that multi-frame channel estimation is more effective in the full duplex FDD scenario, because successive received frames are adjacent (so that denser pilots can be used), but there is still some improvement for half duplex FDD. Similar conclusions can be obtained for the averaged MSE performance of full bandwidth systems (shown in Fig. 6.9 and Fig. 6.8 
for comparison), chunk size (or 2B-IFDMA) and B-IFDMA ${ }^{6}$ using non-iterative and iterative channel estimators.

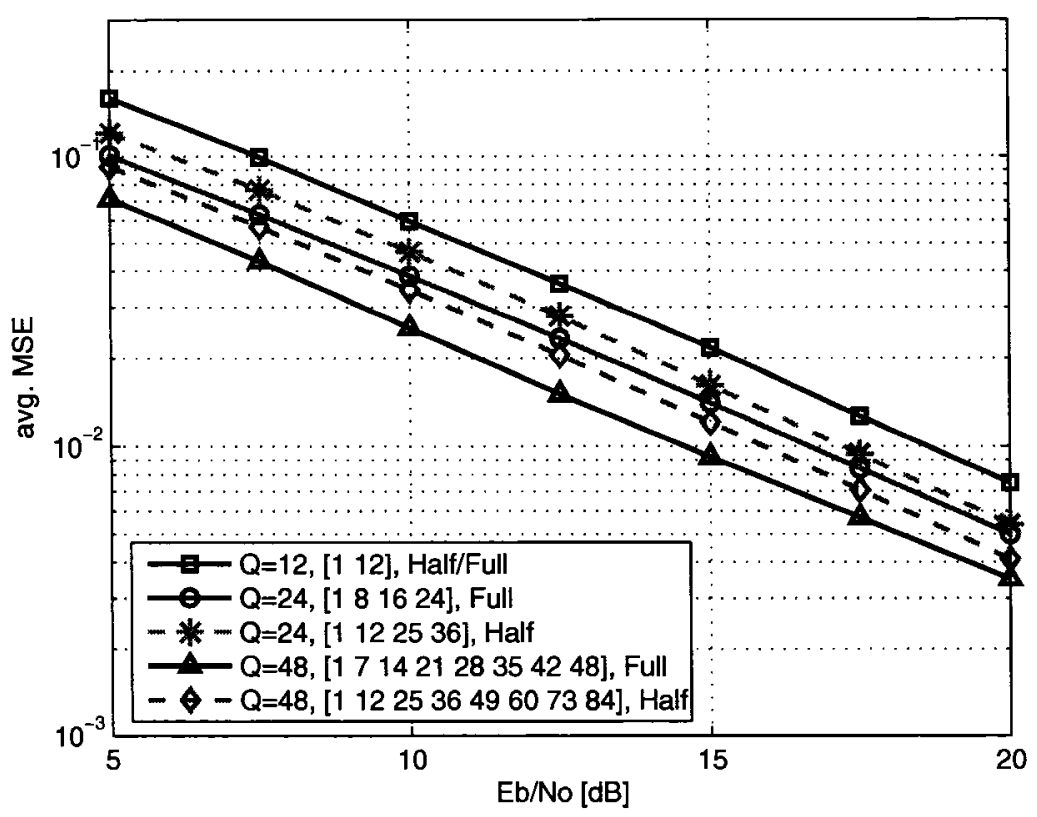

Figure 6.7: Average Mean Square Non-iterative Channel Estimation Error for IFDMA for Estimation over Several Frames in Half and Full Duplex Cases

\subsubsection{Effects of Vehicle Speed on MSE Performance of B- IFDMA Systems over Multiple Frames}

We know that the pilot spacing is upper bounded by (2.47). For a vehicle speed of $240 \mathrm{~km} / \mathrm{hr}, f_{c}=3.7 \mathrm{GHz}$ and $T_{s}=28.8 \mu s, D_{t} \leq 21$. Consider the pilot scheme in Fig. 6.5 (b). The pilot spacing is 24 , which is slightly larger than the maximum pilot spacing allowed by the sampling theorem for a vehicle of $240 \mathrm{~km} / \mathrm{hr}$. In this case, the MSE of the channel estimation would be higher, as shown in Fig. 6.11, where the maximum delay spread and the vehicle speed are known for the calculation of the Wiener filter taps. However, increasing the vehicle speed from $v=80 \mathrm{~km} / \mathrm{hr}$ to $v=200 \mathrm{~km} / \mathrm{hr}$ only results in slightly increasing in MSE performance of the channel

\footnotetext{
${ }^{6}$ Note that the MSE performance for chunk size (or 2B-IFDMA) and B-IFDMA systems are not shown here for simplicity.
} 


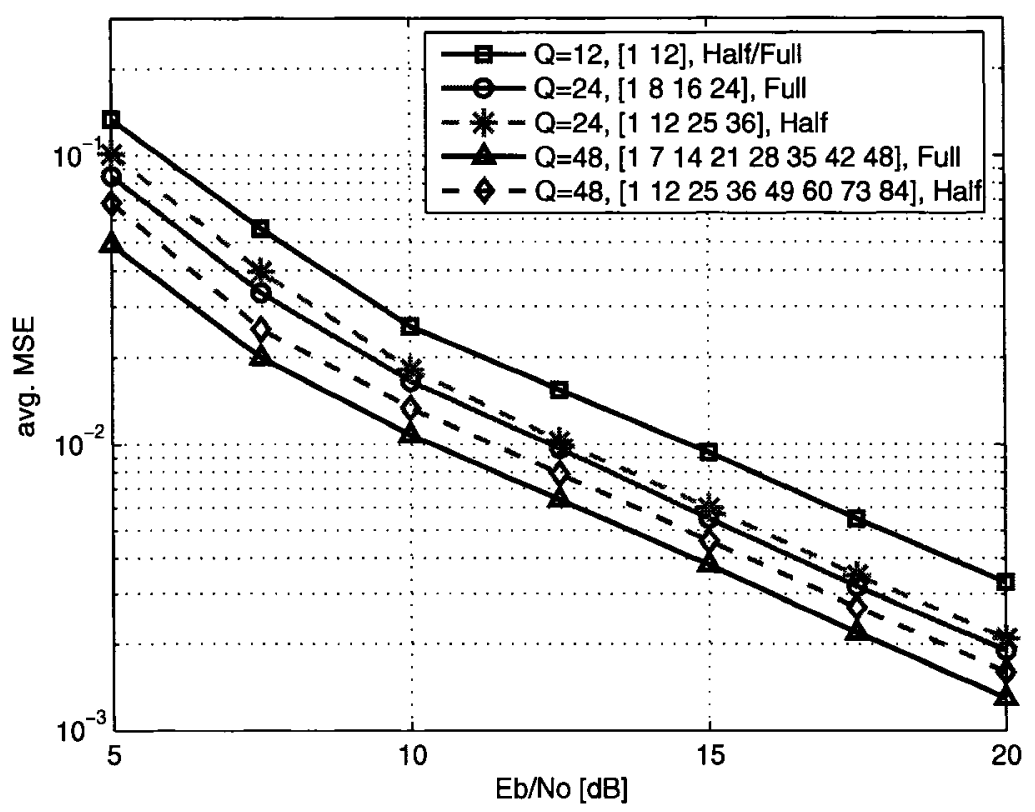

Figure 6.8: Average Mean Square Iterative Channel Estimation $(\lambda=0.3)$ Error for IFDMA for Estimation over Several Frames in Half and Full Duplex Cases

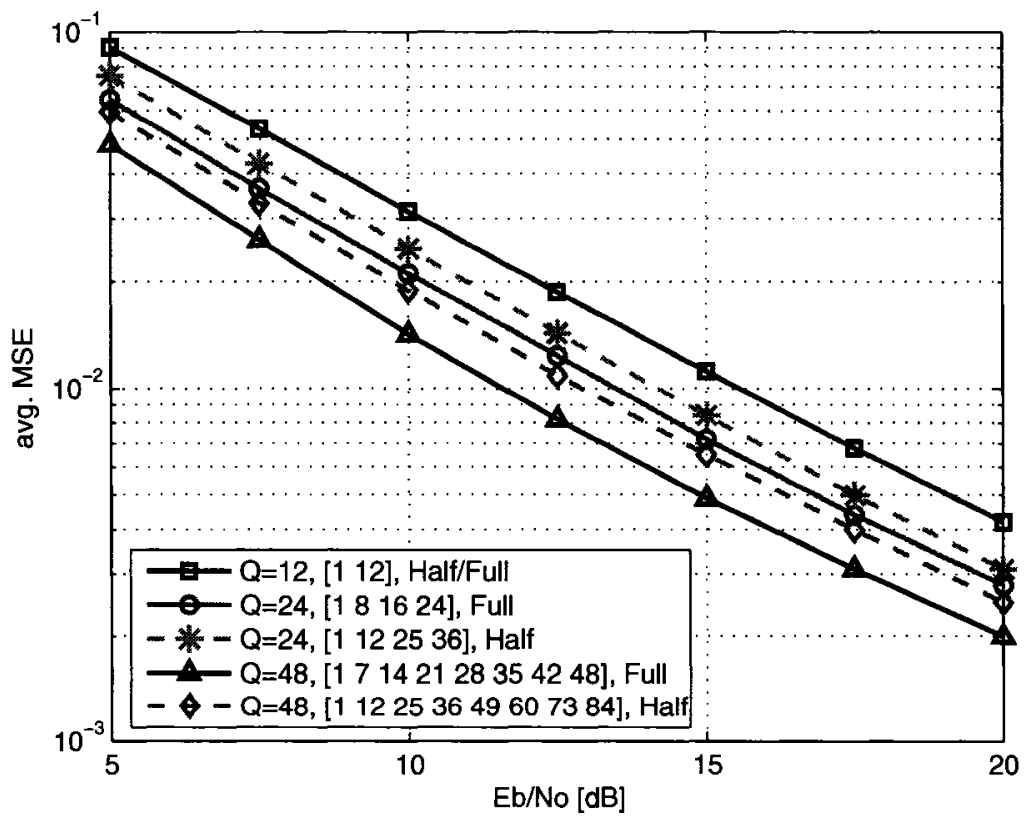

Figure 6.9: Average Mean Square Non-iterative Channel Estimation Error for Full Bandwidth Systems for Estimation over Several Frames in Half and Full Duplex Cases 


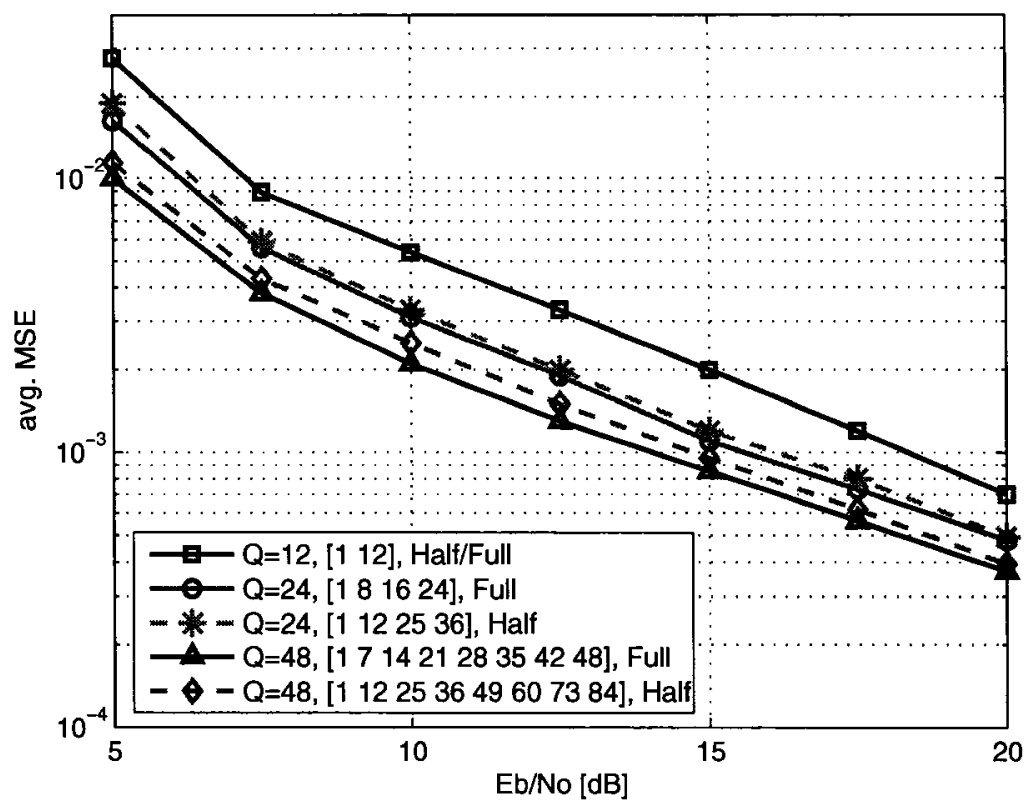

Figure 6.10: Average Mean Square Iterative Channel Estimation $(\lambda=0.3)$ Error for Full Bandwidth Systems for Estimation over Several Frames in Half and Full Duplex Cases

estimation for both non-iterative and iterative channel estimators.

\subsubsection{Frame Error Rate (FER)}

\section{Chunk Size Systems}

Fig. 6.12 shows the FER performance of FDM pilots using frequency domain IBSDFE equalizer with non-iterative channel estimations over one frame and Fig. 6.13 with the proposed frequency domain IDDCE $(\lambda=0.3)$ channel estimation over one frame for the 5-user chunk size systems, described in Sec. 6.2. The performance degradation with respect to that of known CSI, at FER $=10^{-2}$, for $2 \times 1 \mathrm{D}$ Wiener filtering is about $2 \mathrm{~dB}$ for FET and $3 \mathrm{~dB}$ for FDSPT pilots, respectively. Using the low complexity LLSLF estimator in time and frequency direction requires additional $0.5 \mathrm{~dB}$ of SNR for the same FER. Note however that the LLSLF estimator does not require any channel statistics. Using the proposed frequency domain IDDCE $(\lambda=0.3)$, the performance degradation reduces about $0.5 \mathrm{~dB}$ when the decoder is not used and about $1.5 \mathrm{~dB}$ 


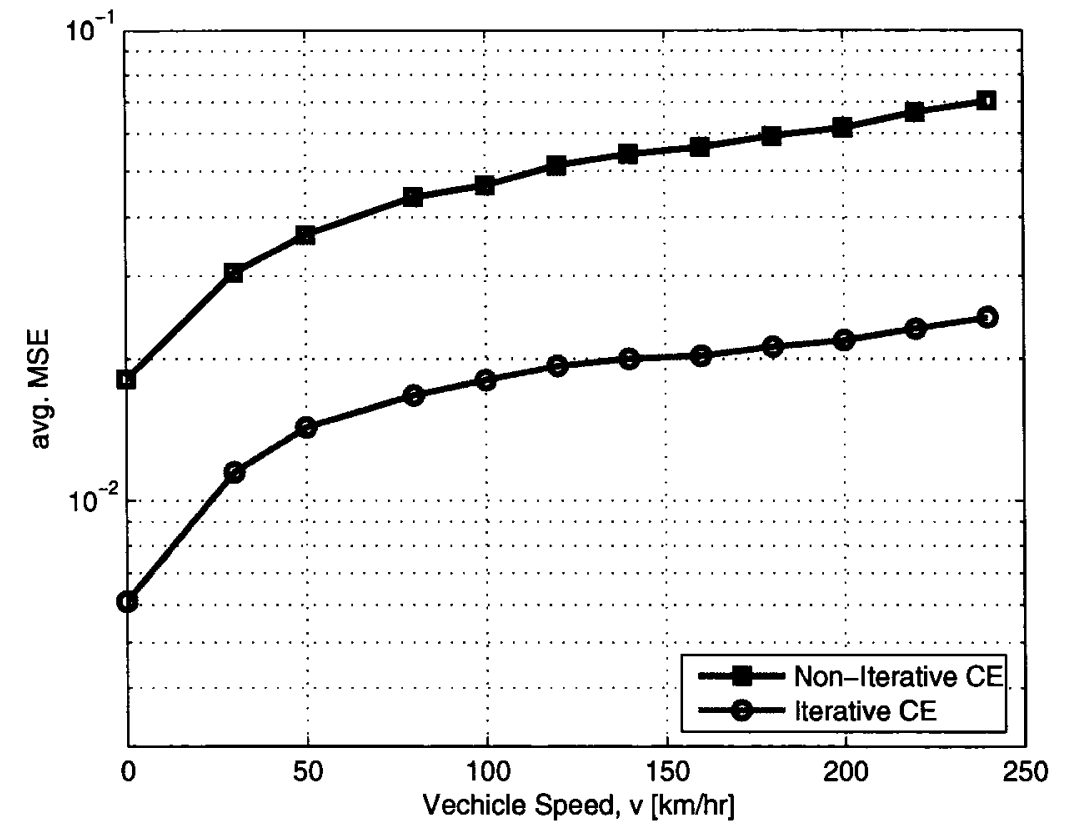

Figure 6.11: Effects of Vehicle Speed on Averaged MSE of Non-iterative and Iterative Channel Estimation for B-IFDMA System over $Q=12$ Half Duplex Frames, $E_{b} / N_{o}=12$ $\mathrm{dB}$ and Pilot Arrangement $=\mathrm{F} 2 \mathrm{~T} 1$ (Parameters Set 2-B).

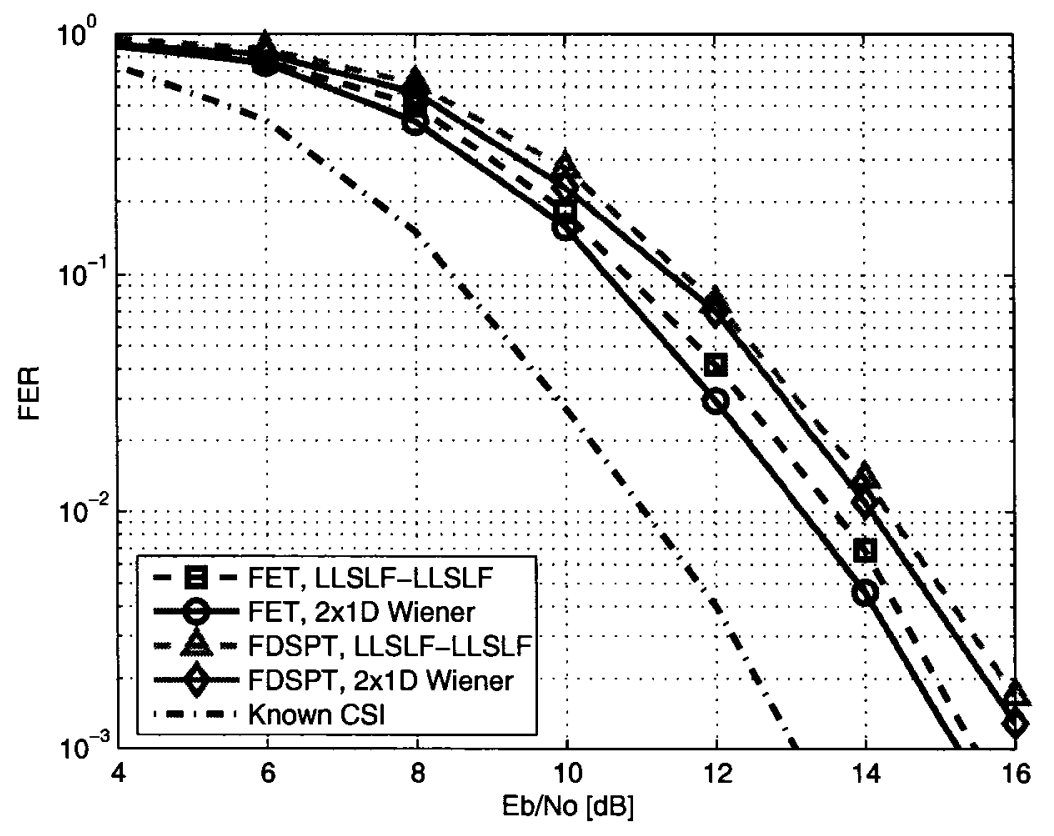

Figure 6.12: FER Performance of FDM Pilots for Coded Systems using Frequency Domain IBSDFE Equalizer with Non-Iterative Channel Estimations over One Frame for Chunk Size Systems (Parameters Set 2-A) 


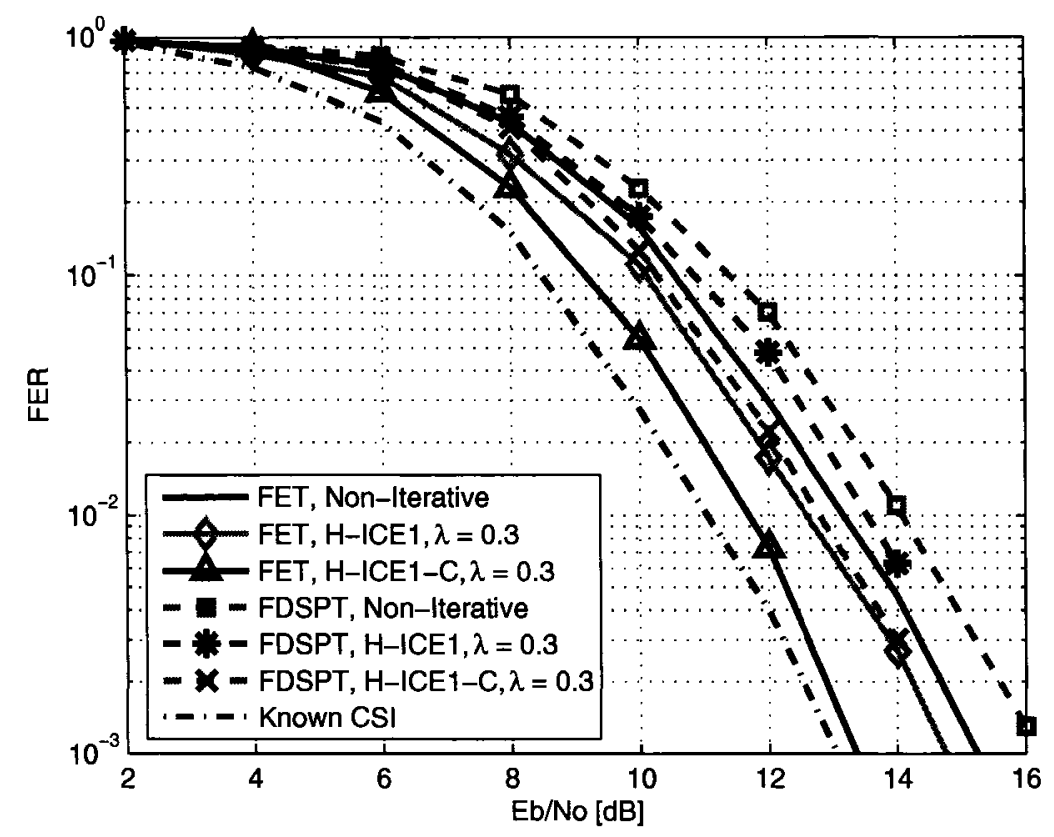

Figure 6.13: FER Performance of FDM Pilots for Coded Systems using Frequency Domain IBSDFE Equalizer with Iterative Channel Estimations over One Frame for Chunk Size Systems (Parameters Set 2-A)

when the decoder is used. Notice that using FET and frequency domain IDDCE $(\lambda=0.3)$ with decoding, the FER performance is about $0.5 \mathrm{~dB}$ away from the FER performance with known CSI, while for FDSPT the performance is about $1.8 \mathrm{~dB}$ away from the performance with known CSI. The results for chunk size systems also suggest that coding/decoding should be used in the iteration process when using the proposed frequency domain IDDCE.

\section{Sub-chunk Size Systems}

For simulation results shown in Fig. 6.14 to Fig. 6.18, parameters Set 2-B and FET pilots are used and we assume the maximum actual delay echo is equal to the $\mathrm{CP}$ length $(3.2 \mu s)$ and the vehicle speed is assumed to be $100 \mathrm{~km} / \mathrm{hr}$, which is mismatched to the actual vehicle speed of $50 \mathrm{~km} / \mathrm{hr}$. FER for IFDMA systems with non- 
iterative and iterative channel estimation $(\mathrm{ICE})^{7}$ is shown in Fig. 6.14, where $Q$ is the number of OFDM symbols used for channel estimation interpolation and smoothing. Also shown for comparison is the case of known channel state information (indicated by "K-CSI"). Using ICE gives roughly a $1 \mathrm{~dB}$ improvement over the corresponding non-iterative channel estimation, and smoothing over 4 frames $(Q=48)$ gives about 0.6 to $1 \mathrm{~dB}$ improvement over smoothing over just one frame. The FER performance of 4-frame smoothing combined with ICE is about $1 \mathrm{~dB}$ worse than the case of known channel state information.

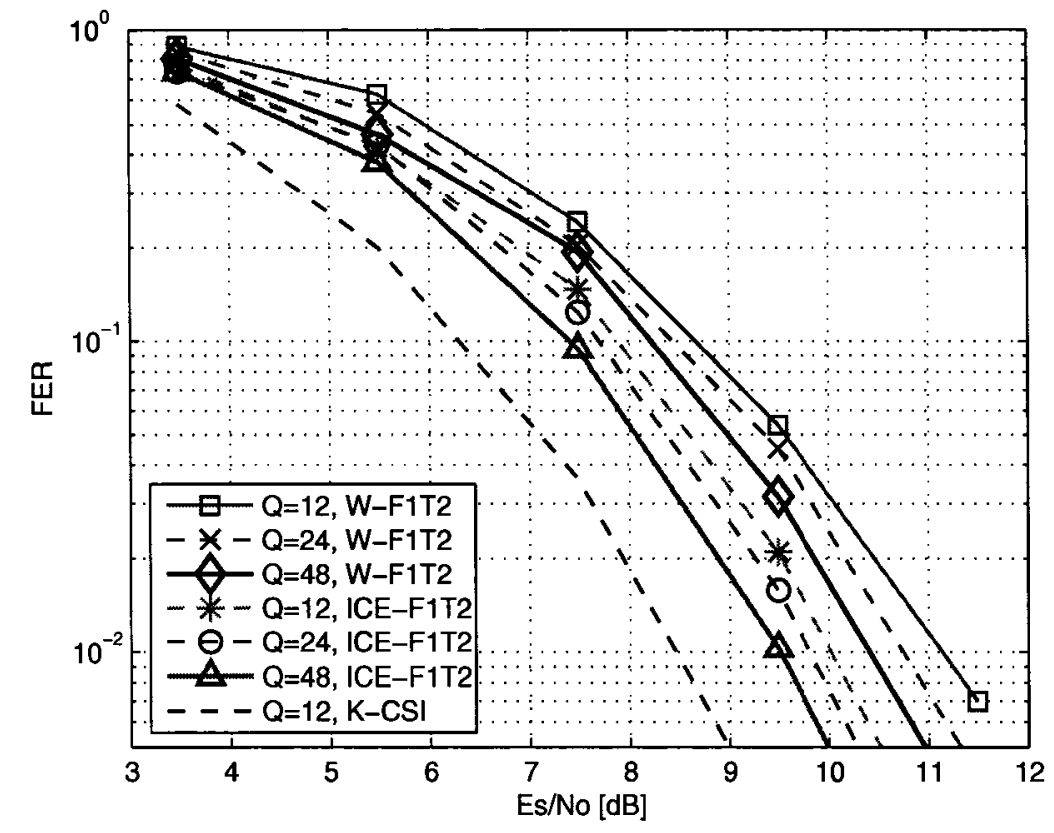

Figure 6.14: FER for IFDMA with FET Pilot Arrangement F1T2, with Non-iterative (W) and Iterative (ICE) Channel Estimation over Multiple Half Duplex Frames (Parameters Set 2-B)

FER for multi-frame smoothing with the F1T1 pilot pattern for B-IFDMA is shown in Fig. 6.15. B-IFDMA is just slightly $(\sim 0.1 \mathrm{~dB})$ better than IFDMA for known $\mathrm{CSI}^{8}$, but with channel estimation with $\mathrm{F} 1 \mathrm{~T} 1$, it is $0.6 \mathrm{~dB}$ to almost $2 \mathrm{~dB}$

\footnotetext{
${ }^{7}$ Note that the iterative channel estimation (ICE) in Fig. 6.14 to Fig. 6.18 refers to the proposed frequency domain IDDCE with $\lambda=0.3$.

${ }^{8}$ The FER for IFDMA with known CSI is not shown in Fig. 6.15 for simplicity.
} 
worse than the corresponding IFDMA, since B-IFDMA with F1T1 has only one pilot per block. Under the simulation conditions $\mathrm{C} 2$ channel with $50 \mathrm{~km} / \mathrm{hr}$, the channel is not constant over a $4 \times 3$ block. Note that although F1T1 incurs less pilot overhead than F2T1 - 1/12 instead of 2/12, it is considerably more than the full bandwidth system pilot overhead of $4 / 96$, where the pilot spacings in frequency and time are 4 and 12, respectively. Fig. 6.16 shows corresponding results for B-IFDMA with

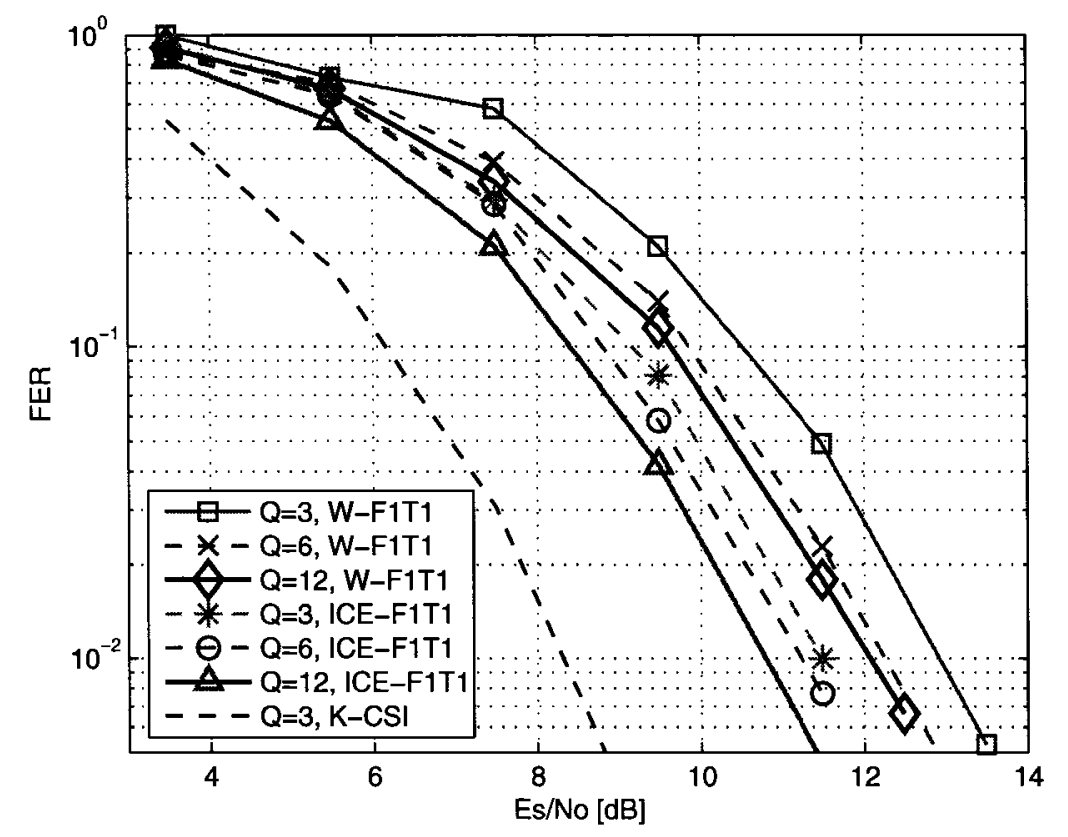

Figure 6.15: FER for B-IFDMA with FET Pilot Arrangement F1T1, with Non-iterative (W) and Iterative (ICE) Channel Estimation over Multiple Half Duplex Frames (Parameters Set 2-B)

the F2T1 FET pilot arrangement (same overhead ratio as for the IFDMA case). BIFDMA still shows up worse than IFDMA with each type of channel estimation, but the difference is less than for the F1T1 case.

The "full-chunk" case, 2B-IFDMA, where fully-occupied chunks $\left(N_{c}=N_{f}=8\right.$ subcarriers per OFDM symbols per chunk) are separated by 32 subcarriers, is shown in Fig. 6.17. The pilot overhead (4/96) is much less than that of the IFDMA and B-IFDMA cases, and the FER performance for ICE with smoothing over 4 frames 


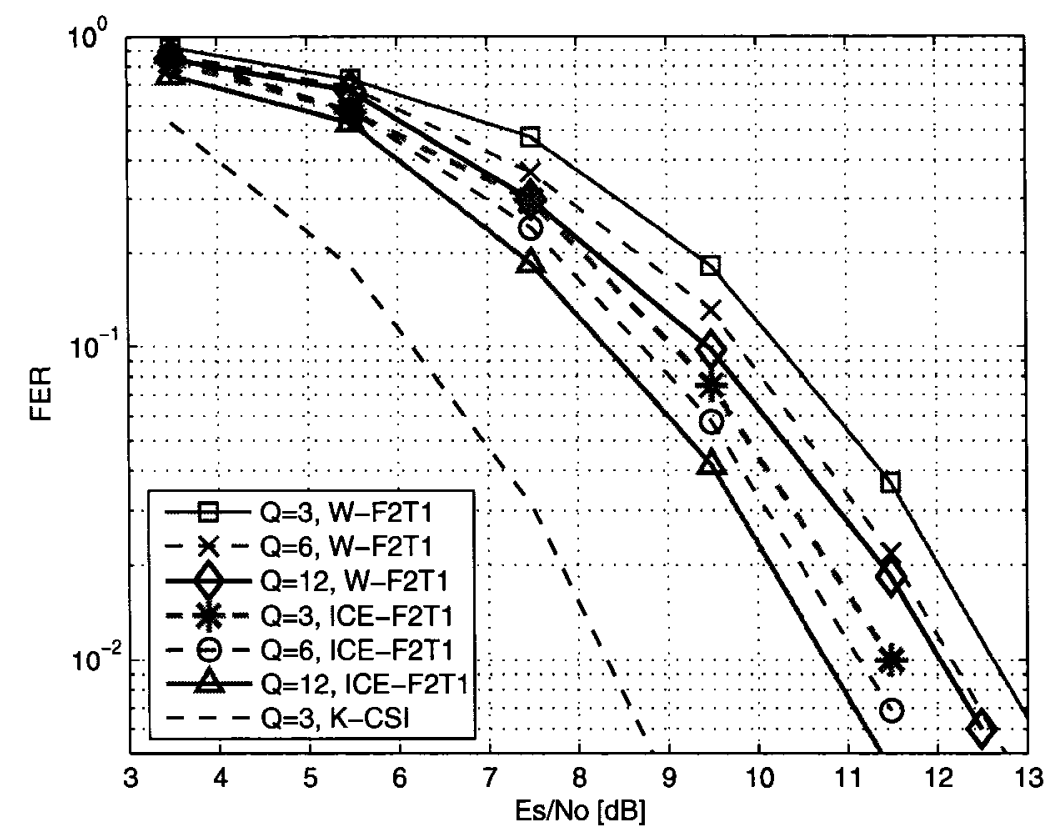

Figure 6.16: FER for B-IFDMA with FET Pilot Arrangement F2T1, with Non-iterative (W) and Iterative (ICE) Channel Estimation over Multiple Half Duplex Frames (Parameters Set 2-B)

is about the same as that of IFDMA with the same channel estimation method $^{9}$. In general, the FER performance loss due to imperfect channel estimation is shown to be more for B-IFDMA than for IFDMA, full chunk size and full bandwidth systems. Finally, the full bandwidth case, in which a user occupies the entire system bandwidth, is shown in Fig. 6.18. The known-CSI performance is slightly worse (about $0.8 \mathrm{~dB}$ ) than that of IFDMA because of more fading correlation among the subcarriers used, but the performance with ICE and 4-frame smoothing is about the same as that of IFDMA. The diversity gains achieved by IFDMA and B-IFDMA are somewhat diminished by their less effective channel estimation capabilities (due to sparse pilot locations) in the $\mathrm{C} 2$ channel with $50 \mathrm{~km} / \mathrm{hr}$.

\footnotetext{
${ }^{9}$ Note that comparison of full bandwidth and 2B-IFDMA cases with IFDMA and B-IFDMA is imprecise, since each of these cases involves different numbers of data symbols, and hence different code block sizes.
} 


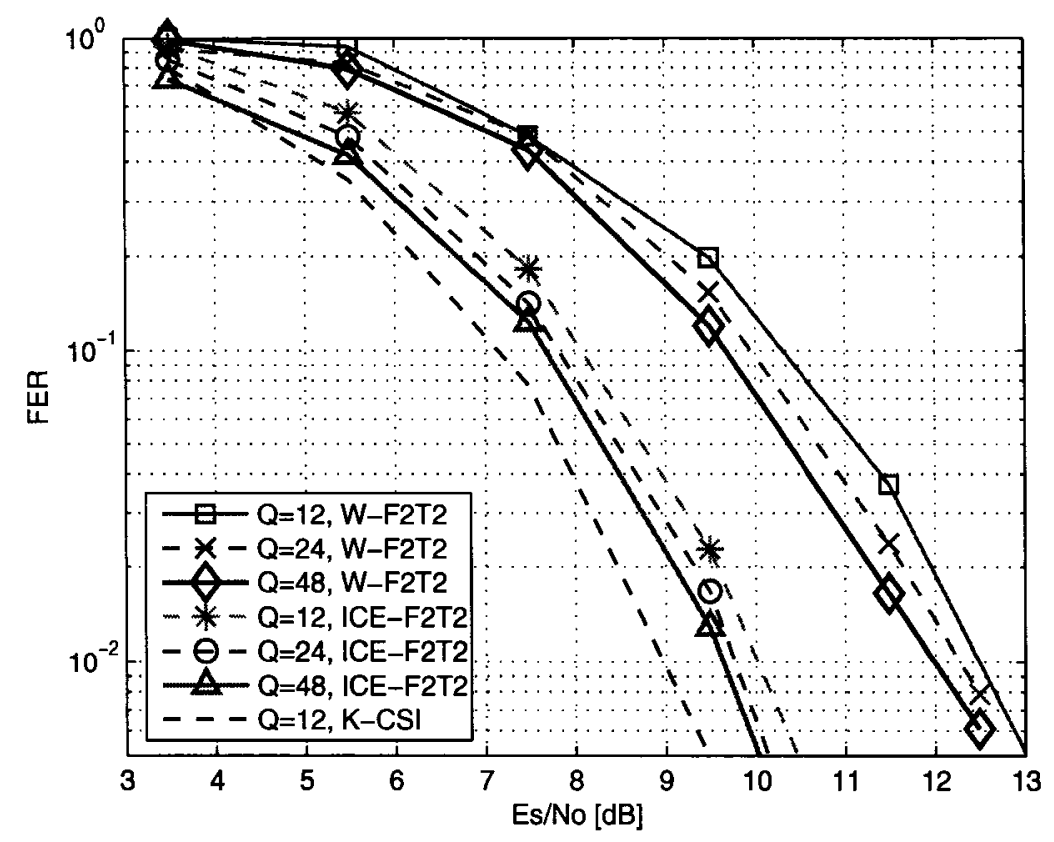

Figure 6.17: FER for 2B-IFDMA with FET Pilot Arrangement F2T2, with Non-iterative (W) and Iterative (ICE) Channel Estimation over Multiple Half Duplex Frames (Parameters Set 2-B)

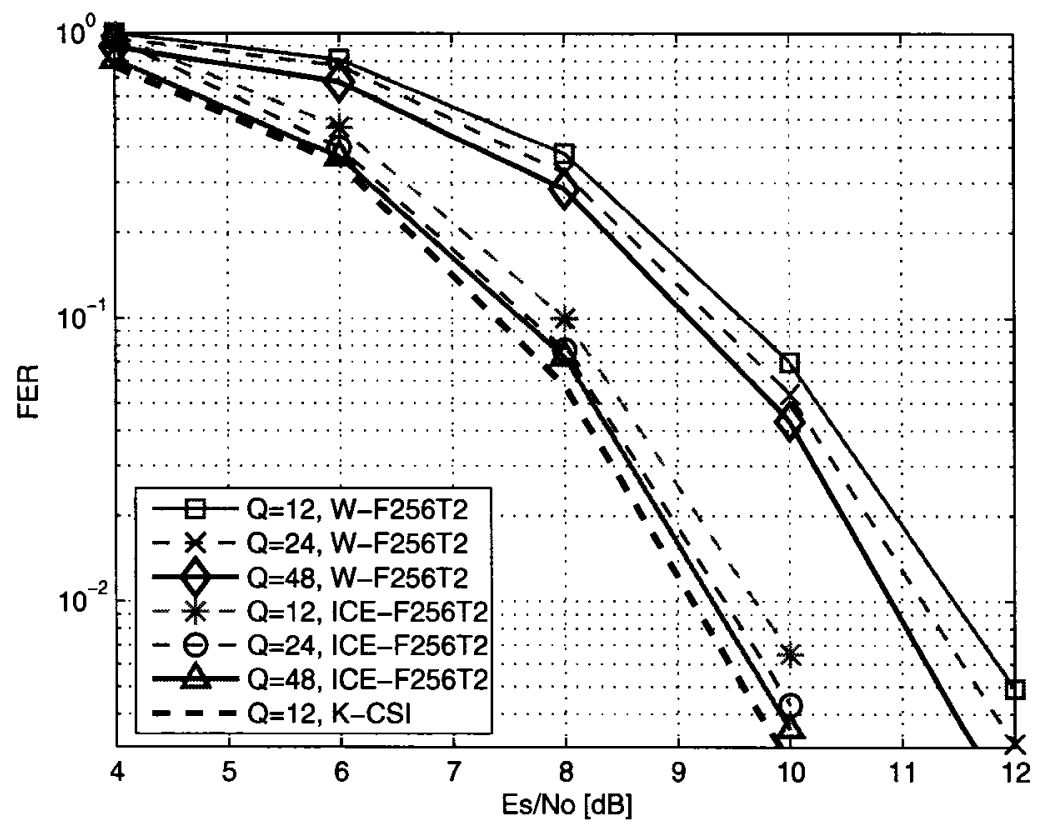

Figure 6.18: FER for Full Bandwidth with FET Pilot Arrangement F256T2, with Noniterative (W) and Iterative (ICE) Channel Estimation over Multiple Half Duplex Frames (Parameters Set 2-B) 


\subsection{Summary}

The channel estimation techniques for full bandwidth systems can be used for chunk and sub-chunk (IFDMA and B-IFDMA) size systems with various FDM pilot arrangements. We evaluated the MSE performance of LLSLF, $2 \times 1 \mathrm{D}$ Wiener filtering and the frequency domain IDDCE with FR algorithm with and without inclusion of a decoder. Similar to the full bandwidth case, it was found that the proposed frequency domain IDDCE with decoder gives the best MSE performance. The advantage of $2 \times 1$ D Wiener filtering over LLSLF diminishes when the number of filter taps is small. In general, the MSE performance of the channel estimation for chunk and sub-chunk size systems using FDM pilots is worse than that of the full bandwidth systems.

Moreover, we showed that using multiple half or full duplex FDD frames can further improve the performance channel estimation for full bandwidth, chunk and sub-chunk size systems. The effectiveness of multi-frame channel estimation depends on the pilot spacing in time, which is upper bounded by the sampling theorem. The FER performance loss due to imperfect channel estimation is shown to be more for B-IFDMA than for IFDMA, full chunk size and full bandwidth systems. The diversity gains achieved by IFDMA and B-IFDMA are somewhat diminished by their less effective channel estimation capabilities (due to sparse pilot locations) in the $\mathrm{C} 2$ channel with $50 \mathrm{~km} / \mathrm{hr}$. 


\section{Chapter 7}

\section{PAPR Reduction using FDM Pilot Sequences}

In this chapter, we investigate the feasibility of applying a PAPR reduction method using pilot sequences, originally proposed for OFDM signals [109], for SC signals with FDM pilots. The idea is to select the FDM pilot sequence for which the transmitted signal produces the lowest PAPR. We also investigate the applicability of the sum of square error (SSE) selection rule for high order modulation of SC signals [110]. The SSE rule selects the pilot sequence which produces the minimum SSE between the transmitted signal and a pre-defined threshold, proportional to the saturation level of a high power amplifier (HPA). The effects of out-of-band radiation for SC and OFDM signals with PAPR reduction using FDM pilot sequences after HPA are also included.

\subsection{Introduction}

From Chapter 4, we know that the multiplexing of FDM pilots for SC modulation induces higher PAPR than that of the SC signal without FDM pilots, or with TDM pilots [6]. Although the PAPR of the SC signal with FDM pilots is still lower than that of an OFDM signal, it is desirable to reduce the PAPR of an SC signal with FDM pilots in order to reduce the power backoff requirement of the high power amplifier (HPA). It is intuitive to consider using the existing techniques for PAPR reduction of an OFDM signal. The issue of high PAPR is common for OFDM and there are many 
well-known methods to reduce the high PAPR of OFDM signals in the literature, such as deliberately clipping and filtering of the signal [105], the partial transmit sequence technique [106], the selected mapping technique [107], the tone reservation technique [108], etc. In [109], the authors proposed a method of reducing the PAPR for OFDM signal with orthogonal pilot sequences (Walsh-Hadamard sequences) by selecting the pilot sequence such that the OFDM signal with pilots gives the lowest PAPR. In this chapter, we want to use this method to reduce the high PAPR of the SC signal with FDM pilots. First, we investigate the feasibility of using orthogonal Walsh-Hadamard (W-H) sequences as the pilot sequences for SC modulation. Second, orthogonal cyclic shifted Chu (CS-Chu) sequences [112] are used to replace the W-H sequences and their PAPR reduction capability for both SC and OFDM systems is investigated. Finally, a different selection rule for SC modulation, based on sum of squared errors (SSE) [110], is used to compare its PAPR reduction capability with that of using the minimum PAPR selection rule. The pilot-aided PAPR reduction for $\mathrm{SC}$ signals is described next.

\subsection{Pilot-Aided PAPR Reduction for SC Signals}

The idea of pilot-aided PAPR reduction for SC signals is similar to that for OFDM signals [109]. Fig. 7.1 depicts the basic idea of PAPR reduction using pilot sequences. $N_{s}$ is defined as the number of pilot sequences for selection, i.e. $N_{s}$ is the selection size. The signal produced by the generalized multi-carrier (GMC) modulator [4] at the $k$ th branch can be written as,

$$
\mathbf{x}^{(k)}=\mathbf{W}_{L \times M}^{H} \mathbf{X}^{(k)}
$$

where $\mathbf{W}_{L \times M}$ is the $L \times M$ discrete Fourier transform (DFT) matrix [111], $L=M I$ is the number of samples per transmission block, $I$ is the upsampling factor, ${ }^{H}$ denotes Hermitian transpose and $\mathbf{X}^{(k)} \in \mathbb{C}^{M \times 1}$ contains the frequency samples of the data and $k$ th pilot sequence. Depending on the pilot arrangement schemes, $\mathbf{X}^{(k)}$ can be 


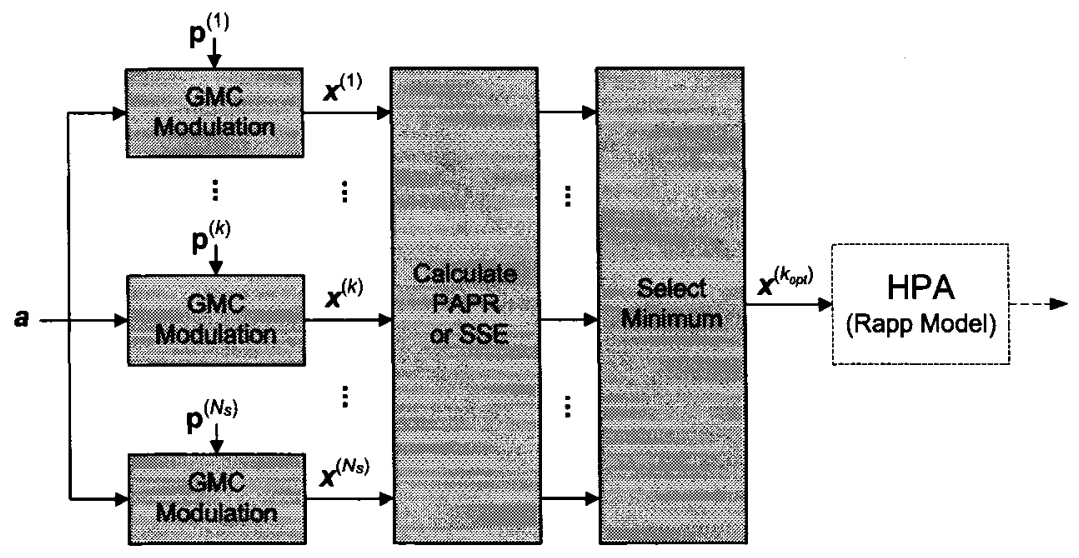

Figure 7.1: Block Diagram of PAPR Reduction using Pilot Sequences (a: data sequences; $\mathbf{p}^{(k)}: k$ th pilot sequences; $N_{s}$ : number of pilot sequences to be selected)

defined as, for FET pilots (see Section 4.2.2),

$$
\mathbf{X}^{(k)}=\left[P_{0}^{(k)}, A_{0}, \ldots, A_{K-2}, P_{1}^{(k)}, A_{K-1}, \ldots, A_{M-N-1}\right]^{T}
$$

and for FDSPT pilots (see Section 4.2.1)

$$
\mathbf{X}^{(k)}=\left[P_{0}^{(k}, A_{1}, \ldots, A_{K-1}, P_{1}^{(k)}, A_{K+1}, \ldots, A_{M-1}\right]^{T}
$$

where $\mathbf{A}=\left[A_{0}, A_{1}, \ldots, A_{M-1}\right]$ is either the DFT of the data sequence a for SC modulation or the data sequence a for OFDM modulation and $\mathbf{P}^{(k)}=\left[P_{0}^{(k)}, P_{1}^{(k)}, \ldots, P_{N-1}^{(k)}\right]$ is the vector of frequency samples of the $k$ th pilot sequence to be selected. In addition to the sequence of CS-Chu sequences, we consider orthogonal W-H sequences, which are used in [109] for blind detection of the index of the transmitted pilot sequence to remove the required side information for an OFDM system. The CS-Chu sequence has constant amplitude in both time and frequency domains [112]. The constant time domain amplitude makes the CS-Chu sequence a good selection for the pilot sequences to reduce PAPR, as shown in the next section. Since CS-Chu sequences are orthogonal [112], the blind detection technique developed in [109] can also be used. ${ }^{1}$ Using

\footnotetext{
${ }^{1}$ We also evaluated the PAPR reduction capabilities of using Chu sequences with different $r$ values. It was found that using the Chu sequences with different $r$ values produces similar PAPR reduction capabilities as that of using CS-Chu sequences. Note however that side information is required in this case since the Chu sequences with different $r$ values are not orthogonal.
} 
(2.7), a CS-Chu sequence with $k$ steps of cyclic shifts is defined as

$$
\mathbf{c}^{(k)}=\left[c_{k \bmod N}, c_{(k+1) \bmod N}, \ldots, c_{(N+k-1) \bmod N}\right]^{T}
$$

where $\bmod N$ denotes modulo $N$. The $k$ th block of the pilot frequency samples using CS-Chu sequence can then be obtained by taking the $N$-point DFT of (7.4), i.e.

$$
\mathbf{P}^{(k)}=\mathbf{W}_{N \times N} \mathbf{c}^{(k)}
$$

where $k=0,1, \ldots, N-1$. The motivation for considering the $\mathrm{W}-\mathrm{H}$ sequences is to know if they can also be used for SC signals so that the same blind detection technique in [109] can be employed for a SC system.

In addition to the PAPR selection rule, the SSE selection rule (proposed in [110]) is used to choose the optimum pilot sequences among the finite selection set of pilot sequences. The motivation to use the SSE selection rule for SC signals is that the number of peaks in a SC block without FDM pilots is greater than that of an OFDM block [110]. The PAPR of a block is defined as

$$
\chi^{(k)}=\frac{\max \left(\left|x^{(k)}[n]\right|^{2}\right)}{E\left\{\left|x^{(k)}[n]\right|^{2}\right\}}, \quad 0 \leq n \leq L-1
$$

where $L=M I$ is the number of samples per block and $I$ is the upsampling factor. The SSE is defined as [110]

$$
\epsilon^{(k)}=\sum_{n=0}^{L-1}\left|e^{(k)}[n]\right|^{2}
$$

where

$$
e^{(k)}[n]=\left\{\begin{array}{lr}
\left|x^{(k)}[n]\right|-\kappa V_{s a t}, & \left|x^{(k)}[n]\right| \geq \kappa V_{\text {sat }} \\
0, & \text { otherwise }
\end{array}\right.
$$

where $0 \leq \kappa \leq 1$ is a threshold factor, $V_{\text {sat }}$ is the saturation level of the HPA and $x^{(k)}[n]$ is the $n$th sample of the $k$ th branch, defined in (7.1). It was observed in [110] that $\kappa=0.9$ gives the best performance results for a moderate amount of non-linearity up to the saturation level ${ }^{2}$. The mathematical model of the non-linear HPA will be

\footnotetext{
${ }^{2}$ From [110] $\kappa$ can be computed as $\kappa=\sqrt[2 p]{(1 / r)^{2 p_{o}-1}}$, where $r$ (smaller than one) is a constant ratio of the input threshold value (or $\kappa V_{\text {sat }}$ ) to the output threshold value. Note that $V_{\text {sat }}$ is proportional to the input power backoff $\beta$ (see (7.12)).
} 
described in Section 7.5. Depending on the selection rules, the optimum index of the pilot sequence, denoted as $k_{\text {opt }}$, can be found as, for minimum PAPR rule,

$$
k_{\text {opt }}=\arg \min _{k \in\left\{1,2, \ldots, N_{s}\right\}} \chi^{(k)}
$$

and for SSE,

$$
k_{\text {opt }}=\arg \min _{k \in\left\{1,2, \ldots, N_{s}\right\}} \epsilon^{(k)}
$$

where $N_{s}$ is the selection size.

\subsection{PAPR Reduction Capability of Walsh-Hadamard and Cyclic-shifted Chu Sequences}

In the time domain, the transmitted signal $x^{(k)}[n]$ can also be written as the sum of the data and the pilot signals,

$$
\mathbf{x}^{(k)}=\underbrace{\mathbf{W}_{L \times M}^{H} \mathbf{A}}_{\text {data }}+\underbrace{\mathbf{W}_{L \times M}^{H} \mathbf{P}^{\prime(k)}}_{\text {pilot }}
$$

where $\mathbf{A}=\left[0, A_{0}, \ldots, A_{K-2}, 0, A_{K-1}, \ldots, A_{M-N-1}\right]^{T}$ for FET and for FDSPT $\mathbf{A}=$ $\left[0, A_{1}, \ldots, A_{K-1}, 0, A_{K+1}, \ldots, A_{M-1}\right]^{T}$, and $\mathbf{P}^{\prime(k)}=\left[P_{0}^{(k)}, 0, \ldots, 0, P_{1}^{(k)}, 0, \ldots, 0, P_{N-1}^{(k)}, 0, \ldots, 0\right]^{T}$.

It is obvious that if the pilot signal is added destructively to the data signal, the high peaks can be reduced. On the other hand, more high peaks can be generated if the data and pilot signal are added in a way that the resulting magnitude is larger than that of the data signal. The applicability of the W-H sequences for SC signals depends on the high peaks of the non-constant envelope of the time domain signal $\mathbf{W}_{L \times M}^{H} \mathbf{P}^{\prime(k)}$. For W-H sequences, the distributions and the magnitude of the peaks depend on the choice of the particular sequence. The magnitude of the peaks is smaller for smaller values of $N$ and is larger for larger values of $N$. The CS-Chu pilot signal has a relatively constant signal envelope. Its waveform variations are solely due to the oversampling and filtering processes inherent in zero-padding before the IDFT operation. Many high peaks of the W-H pilot signal can cause even more high peaks for both SC and OFDM signals. 
Fig. 7.2 shows the complementary cumulative distribution function (CCDF) of the magnitude of $x[n]$, denoted as $|x[n]|$, for $M=64, N=8$ and $I=4$ and Fig. 7.3 for $M=512, N=128$ and $I=4$. Note that no spectrum-shaping filter is used for simplicity. $N_{s}$ is the number of pilot sequences for selection. The FDM pilot scheme is the FET and the selection rule used in the simulation is the PAPR rule ${ }^{3}$. The CCDF of the magnitude of a SC signal without pilots in both figures is included for a benchmark comparison. Moreover, the CCDF of the magnitude of a SC signal with a fixed, i.e. $N_{s}=1$, Chu sequence as the FDM pilots is also included for comparison. For small block size (and thus small value of $N$ ), e.g. $M=64$, the $\mathrm{W}-\mathrm{H}$ sequences can be used to reduce the PAPR of SC signals with FDM pilots and OFDM signals with pilots since the probability of high peaks has been lowered, as shown in Fig. 7.2. However, for large block size, e.g. $M=512$, the use of $\mathrm{W}-\mathrm{H}$ sequences generates high peaks with higher probability than that of using a fixed Chu sequence for both $\mathrm{SC}$ and OFDM signals, as shown in Fig. 7.3 (diamond and solid line without markers). Many high peaks of the time domain W-H pilot signal for large block size account for this undesirable result. Note that in [109] the experiments were conducted only for small block size $(M=64)$ of OFDM signals. We also observe that using CSChu sequences as the pilot sequences can reduce, for small and large block size, the probability of high peaks for both SC and OFDM signals, as shown in Fig. 7.3 (square and cross markers). Since a CS-Chu sequence has a constant envelope in the time domain, fewer peaks will be generated by adding the pilot signals at the expense of more values close to the median.

\subsection{Effects of Selection Size and Rules}

The selection size affects the PAPR reduction capability. The larger the selection set, the better the PAPR reduction capability. However, a larger value of $N_{s}$ means more

\footnotetext{
${ }^{3}$ The FDSPT with PAPR selection rule produces similar results, so its results are not included here.
} 


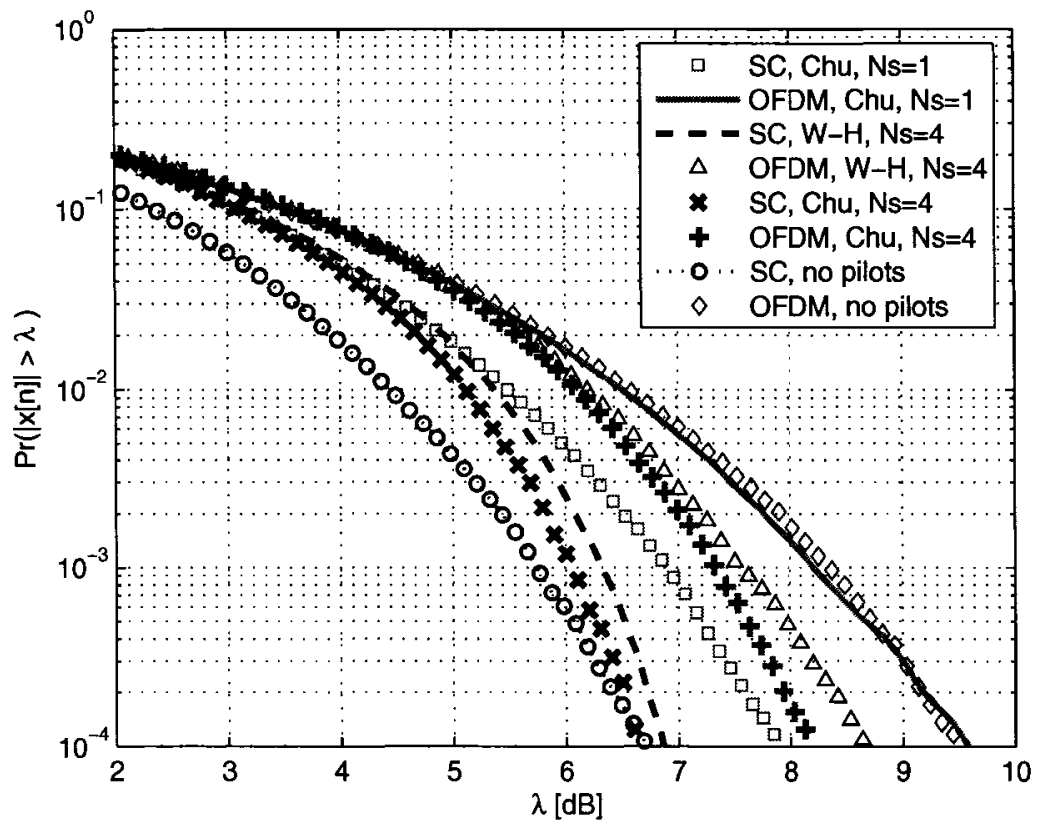

Figure 7.2: CCDF of Magnitude of OFDM and SC Signals with Parameters: $M=64, I=$ $4, N=8$, QPSK, FET, Selection Rule $=$ PAPR, W-H and CS-Chu Pilot Sequences

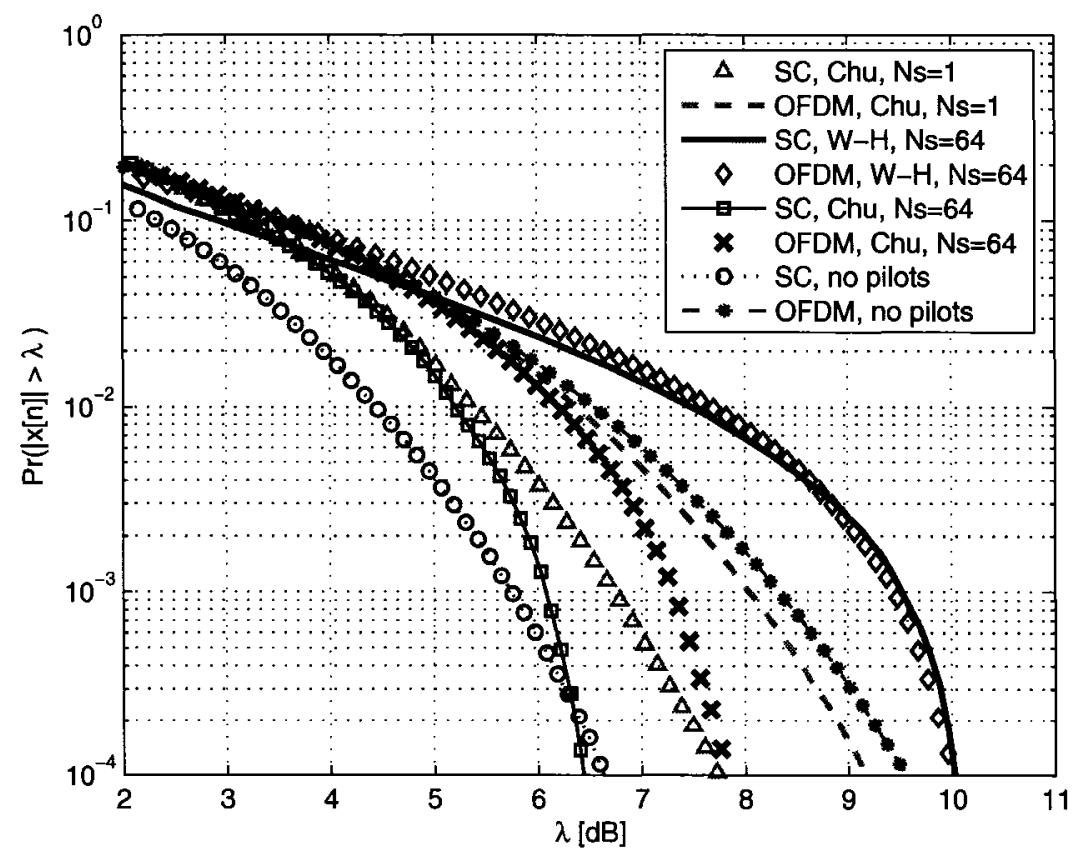

Figure 7.3: CCDF of Magnitude of OFDM and SC Signals with Parameters: $M=512, I=$ $4, N=128$, QPSK, FET, Selection Rule $=$ PAPR, W-H and CS-Chu Pilot Sequences 
complex transmitter and receiver processing and longer processing delays. Fig. 7.4 depicts the CCDF of $|x[n]|$, generated using CS-Chu sequences with $M=416, N=$ 104 and $I=4$ and different values of $N_{s}$. With a selection size of 16, i.e. $N_{s}=16$, the CCDF curve is about $0.4 \mathrm{~dB}$ off that using all the available CS-Chu sequences $\left(N_{s}=104\right)$ with $N=104$ at the probability of $10^{-4}$.

Fig. 7.5 shows the CCDF of $|x[n]|$ generated using CS-Chu sequences and SSE selection rule with different input power backoff (IBO) values, defined as

$$
\beta=-10 \log _{10} \frac{E\left\{\left|v_{\text {in }}\right|^{2}\right\}}{P_{\text {sat }}}
$$

where $\beta$ denotes the IBO in $\mathrm{dB}$ and $P_{s a t} \triangleq\left|V_{\text {sat }}\right|^{2}$ is the power of the saturation level of the amplifier. Note that a higher order modulation scheme (such as 16-QAM) is used since the SSE rule is more efficient when used for higher order SC modulation [110]. With a typical value of IBO, e.g. $\beta=7 \mathrm{~dB}$, the CCDF curve is similar to that of using the PAPR rule. The SSE rule is sensitive to the values of IBO. From (7.7), we know that the SSE rule calculates the SSE with respect to the saturation level $V_{\text {sat }}$. Fewer peaks are used for calculation of SSE for larger values of $V_{\text {sat }}$. On the other hand, from the definition of IBO in (7.12), it is obvious a larger value of $\beta$ gives a larger value of $V_{\text {sat }}$, given the average signal power is fixed.

\subsection{Effects on Out-of-Band Distortion after HPA}

We want to investigate the effects of the HPA on the out-of-band radiation for signals with PAPR reduction and compare with that without the PAPR reduction. The non-linear model for the HPA used in the simulation is the Rapp model [113], whose AM/AM curve has the following relationship,

$$
v_{\text {out }}=\frac{v_{\text {in }}}{\left(1+\left(\frac{v_{\text {in }}}{V_{s a t}}\right)^{2 p_{o}}\right)^{\frac{1}{2 p_{o}}}}
$$

where $v_{\text {in }}$ is the input voltage, $v_{\text {out }}$ is the output voltage, $V_{\text {sat }}$ is the saturation level of the amplifier and $p_{o}$ is the amount of non-linearity up to the saturation level. The 


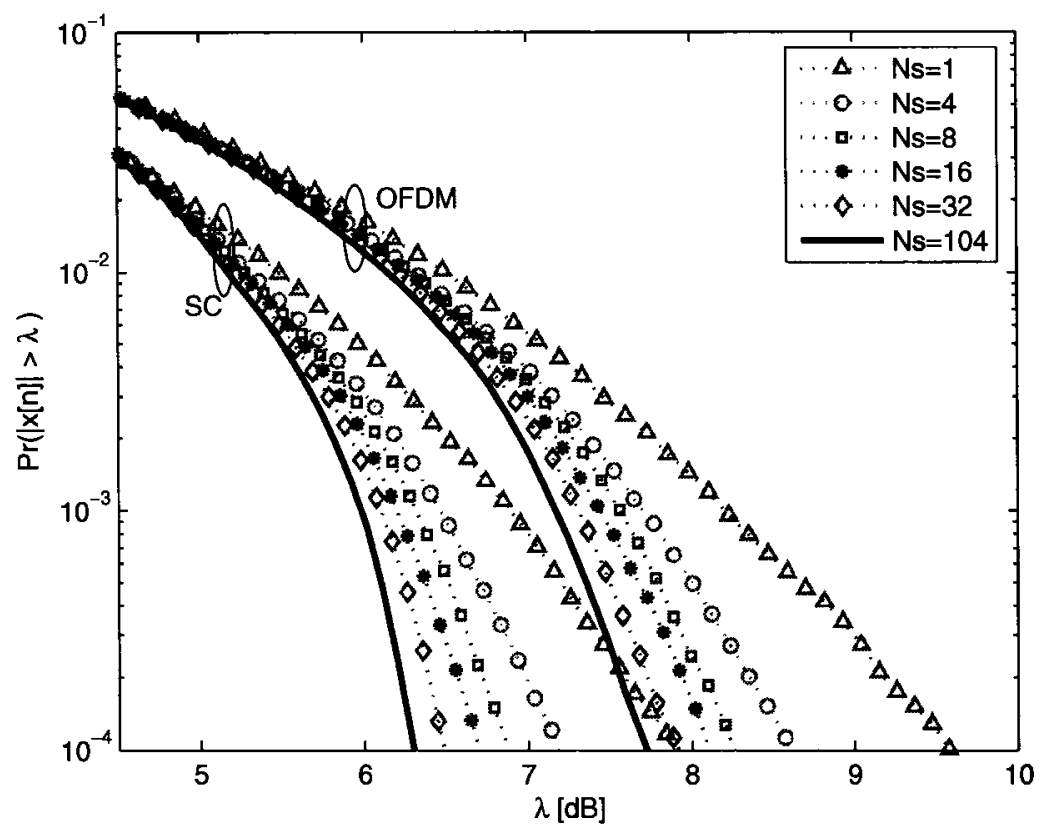

Figure 7.4: CCDF of Magnitude of OFDM and SC Signals with Parameters: $M=416, I=$ 4, $N=104$, QPSK, FET, CS-Chu Pilot Sequences, Selection Rule = PAPR

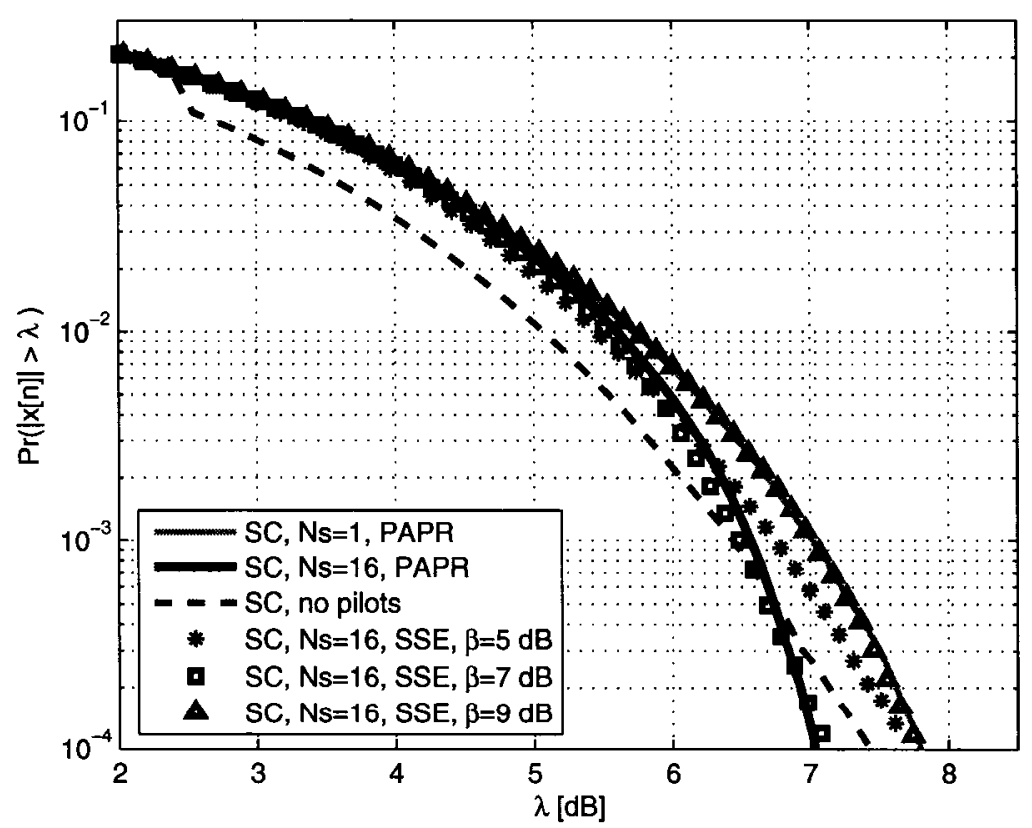

Figure 7.5: CCDF of Magnitude of SC Signals with Parameters: $M=416, I=4, N=104$, 16-QAM, FET, CS-Chu Sequences 


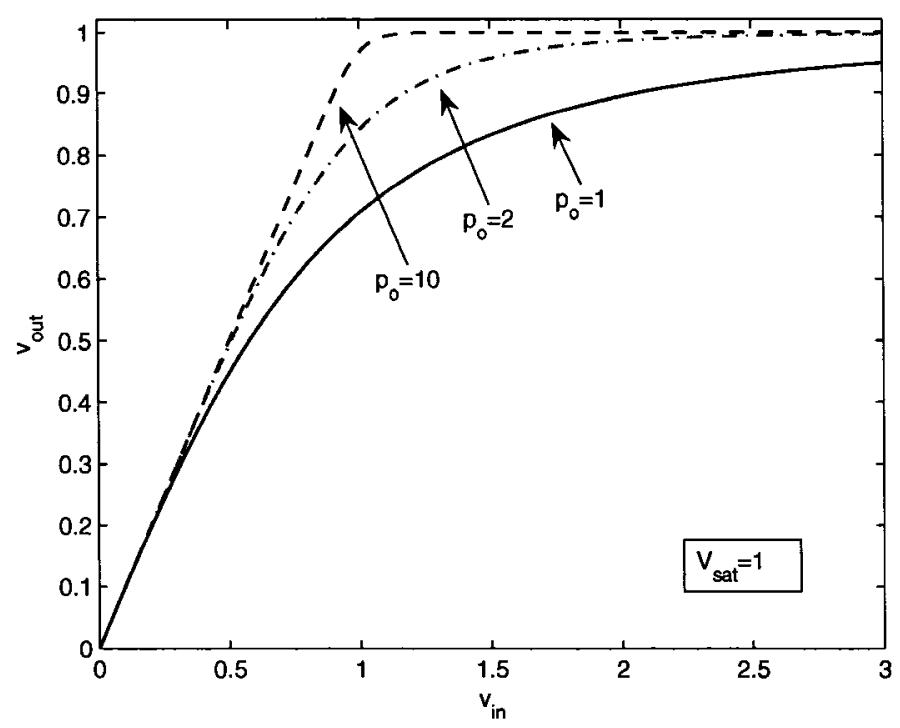

Figure 7.6: AM/AM Curves of Rapp Model with Different Values of $p_{o}$

larger the value of $p_{o}$, the larger the linear region of the curve, as shown in Fig. 7.6. Fig. 7.7 and Fig. 7.8 show the power spectra density (PSD) of the SC signals and OFDM signals with and without pilot sequences for $M=64$ and $M=416$, respectively with $p_{o}=10$. A time domain (TD) window with a rolloff factor of $5.5 \%$ is used to shape the transmitted spectrum. We see that in both cases the out-of-band radiation is reduced for OFDM and SC signals with PAPR reduction using CS-Chu sequences. Fig. 7.9 shows the PSD of the SC and OFDM signals using a HPA with a larger portion of non-linearity, e.g. $p_{o}=2$. There is almost no improvement in terms of out-of-band radiation when the PAPR reduction method is utilized. This can be explained by looking at the CCDF curves in Fig. 7.3. The curves with and without PAPR reduction using pilot sequences almost coincide for $\lambda \leq 5.5 \mathrm{~dB}$. Since the saturation level is $\beta=7 \mathrm{~dB}$, the high peaks will be trimmed by the non-linear portion of the HPA even after the PAPR reduction. The SSE rule produces similar results as that with PAPR rule for high order modulation of SC signals, as shown in Fig. 7.10. 


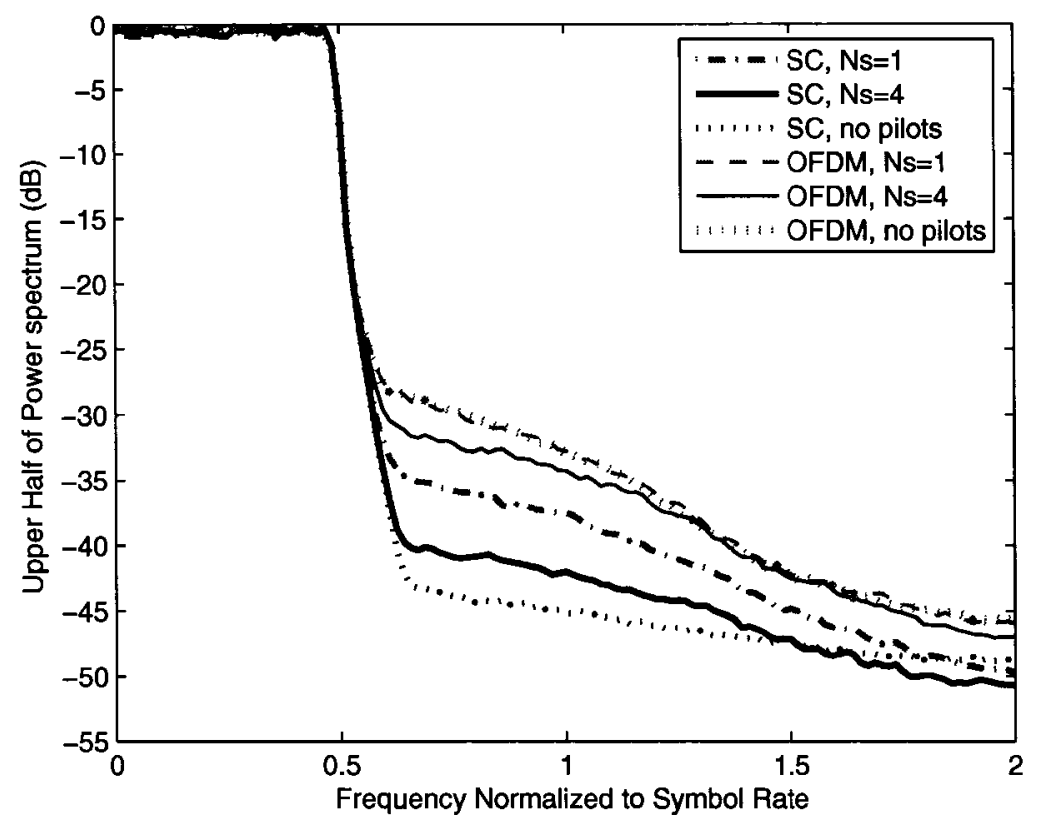

Figure 7.7: PSD of SC and OFDM Signals with Parameters: $M=64, N=8, I=4$, QPSK, FET, CS-Chu Sequences, TD Window Rolloff Factor $=5.5 \%$, PAPR Selection Rule, $p_{o}=10$ and $\beta=5 \mathrm{~dB}$

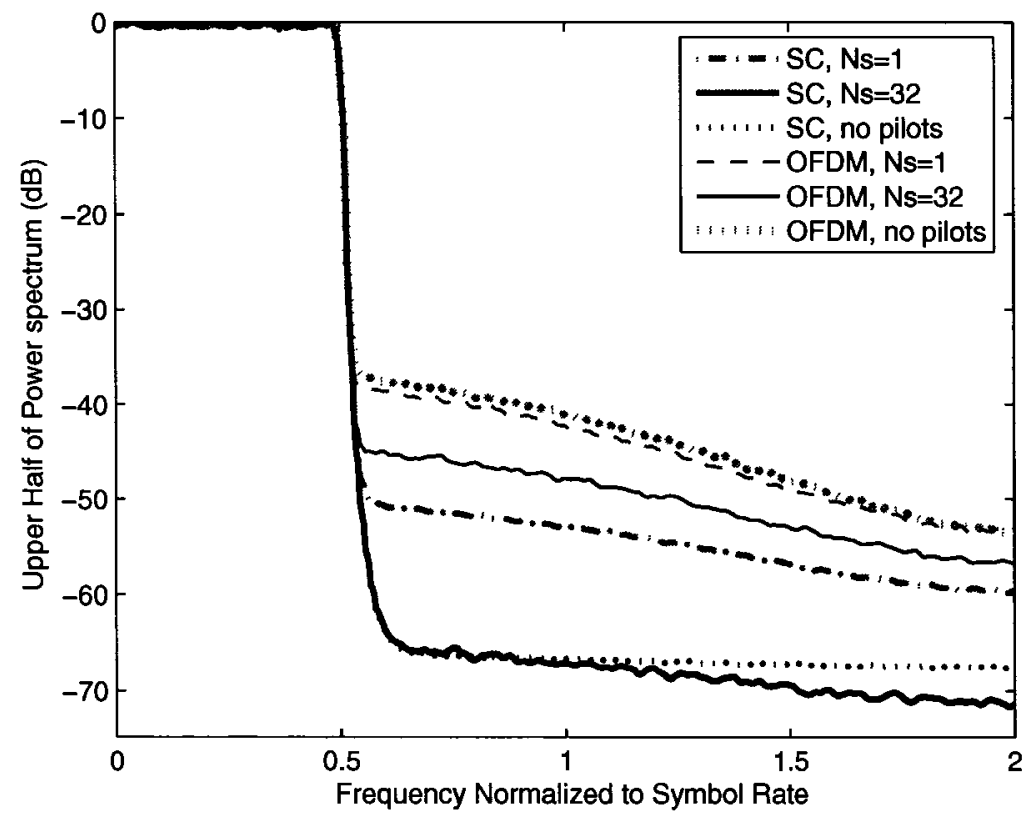

Figure 7.8: PSD of SC and OFDM Signals with Parameters: $M=416, N=104, I=4$, QPSK, FET, CS-Chu Sequences, TD Window Rolloff Factor $=5.5 \%$, PAPR Selection Rule, $p_{o}=10$ and $\beta=7 \mathrm{~dB}$ 


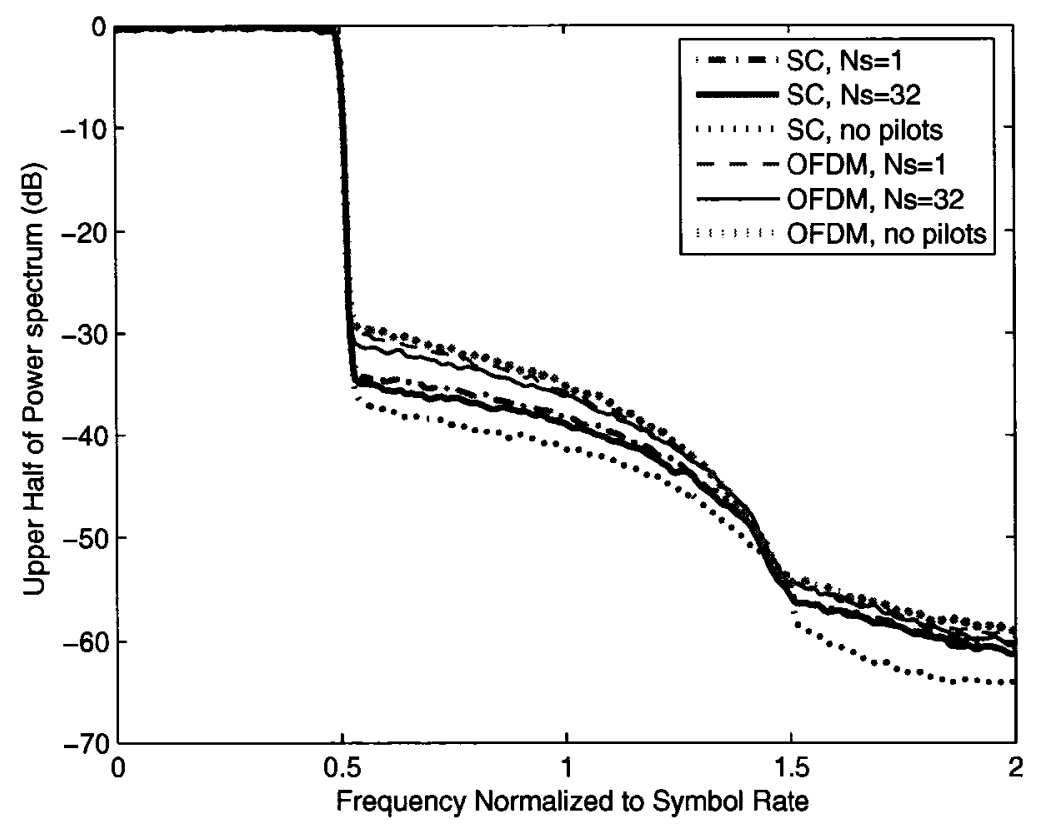

Figure 7.9: PSD of SC and OFDM Signals with Parameters: $M=416, N=104, I=4$, QPSK, FET, CS-Chu Sequences, TD Window Rolloff Factor $=5.5 \%$, PAPR Selection Rule, $p_{o}=2$ and $\beta=7 \mathrm{~dB}$

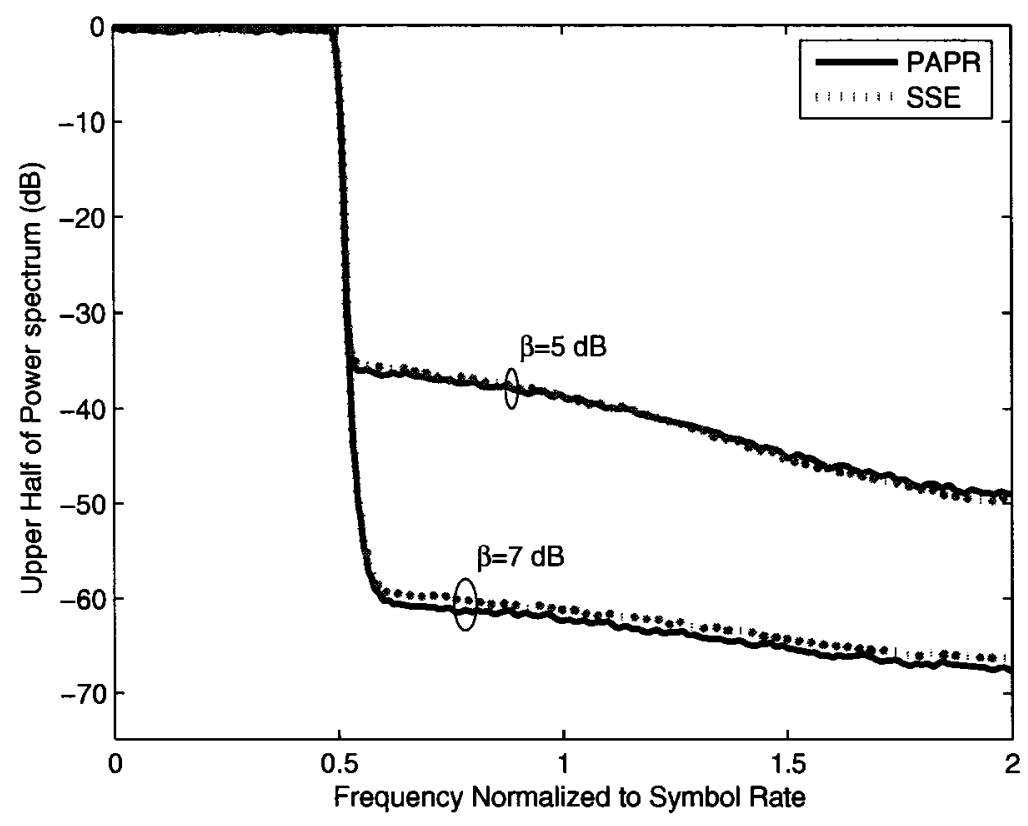

Figure 7.10: PSD of SC Signals with Different Selection Rules $(M=416, N=104, I=4$, 16-QAM, FET, CS-Chu Sequences, TD Window Rolloff Factor $=5.5 \%, N s=32, p_{o}=10$ and $\beta=7 \mathrm{~dB}$ ) 


\subsection{Summary and Discussions}

The feasibility of using the PAPR reduction method proposed in [109] for SC signals with FDM pilots has been investigated. The idea is to select the pilot sequence with the lowest PAPR. We also investigated the applicability of the SSE selection rule, proposed in [110], for high order modulation of SC signals. It was found that the PAPR reduction capabilities of $\mathrm{W}-\mathrm{H}$ sequences and CS-Chu sequences are similar for small block size, e.g. $M=64$, but not for larger block size, e.g. $M=512$. CS-Chu sequences can be used for different block sizes. With an appropriate choice of the value of $\beta$, the SSE selection rule produces similar results as that of PAPR selection rule. The effects of out-of-band radiation for SC and OFDM signals with PAPR reduction after HPA depend on the nonlinear characteristic of the HPA below saturation. Outof-band radiation improvement is obvious for a HPA that approximates an ideal linear clipper $\left(p_{0}=10\right)$. To use PAPR reduction using pilot sequences with blind detection, slightly larger processing delay and implementation complexity in the transmitter and receiver side are required. 


\section{Chapter 8}

\section{Conclusions, Design Recommendations and Future Work}

This chapter provides the conclusions and some recommendations for system designers on frequency domain channel estimation and equalization for SC systems as well as the future work.

\subsection{Conclusions}

To fulfill our main objective of designing and developing FDM pilots for uplink SC systems in time-varying frequency selective fading channels, we developed two types of FDM pilots (FET and FDSPT pilots) using the GMC approach and analyzed their impacts on SC signals in terms of PAPR and power backoff requirements. We also proposed and evaluated (using MSE and FER performance) non-iterative and iterative frequency domain channel estimation techniques for SC signals with FDM pilots. Moreover, we evaluated and compared their performance (BER and FER) with that of an OFDM system with similar pilot scheme and overhead. We also applied and analyzed our proposed non-iterative and iterative channel estimators for chunk and sub-chunk size systems over single and multiple full and half duplex frames.

It was found that the multiplexing of in-band FDM pilots induces higher PAPR and power backoff requirement of an SC signal, comparing with that of an SC signal 
without FDM pilots, although the PAPR and power backoff requirement are still lower than that of an OFDM signal. We then proposed a PAPR reduction technique for SC signals with FDM pilots, using a set of CS-Chu pilot sequences. It was found that out-of-band radiation improvement is obvious for a HPA that approximates an ideal linear clipper. In general, the FER performance of a coded system for our proposed frequency domain channel estimation techniques using TDM and FET pilots are similar, while using FDSPT pilots loses about $1 \mathrm{~dB}$ of SNR with respect to that of using FET or TDM pilots.

To further improve the performance of systems using FDSPT pilots, we proposed a frequency domain decision-directed interference cancellation technique, for which the loss of using FDSPT pilots reduces by $0.5 \mathrm{~dB}$. It was also found that the performance of channel estimation for chunk and sub-chunk size systems is worse than that of a full bandwidth system due to frequency diversity and limited number of available pilots per chunk or sub-chunk. It was shown that multiple half or full duplex frames can be used to improve channel estimation for chunk size, sub-chunk size and full bandwidth systems at the expense of slightly increasing the implementation complexity. In addition to FET pilots, FDSPT pilots can also be used for OFDM systems with channel coding. Taking into account the required power backoff of a HPA, a serial modem with FDM pilots could be designed with a power amplifier with about $2 \mathrm{~dB}$ lower maximum power rating than that of a corresponding OFDM modem.

We also studied, analytically and semi-analytically in the frequency domain, the impact of imperfect channel estimates on the MSE of the equalizer output, which is used to estimate the BER of an uncoded $\mathrm{SC}$ system. It was found that the MSE of the equalizer output with imperfect channel estimate is the sum of the MSE for the known CSI and the scaled MSE of the channel estimates. The results can be used as alternative tools for estimating the BER performance without simulating the complete system. Moreover, the design process of channel estimation and system performance evaluation can be decoupled. 
A comparative study of the performance comparison between IFDMA and DSCDMA systems, with and without carrier frequency offset and Wiener phase noise, was included since IFDMA can be considered as a sub-chunk size system (see Appendix B). For systems with carrier frequency offset and Wiener phase noise the performance degradation of the IFDMA system is sensitive to the number of active users although it outperforms the conventional CDMA system when it is not at full capacity. The performance degradation becomes larger when the frequency offset and phase noise increase. The two systems perform closely at full capacity.

\subsection{Design Recommendations}

From this thesis, we have the following recommendations for designing channel estimators for SC systems using FDM pilots in broadband wireless channels:

1. Frequency domain signal generation of SC signals using the GMC approach facilitates flexible and efficient assignment of signals to available spectrum.

2. Most of the existing frequency domain channel estimation techniques for OFDM systems can be adopted for SC systems ${ }^{1}$.

- It is worth looking into the existing techniques for OFDM systems before designing similar frequency domain techniques for SC systems as there are many matured frequency domain techniques for OFDM systems. One should always bear in mind that in frequency domain the envelope of a SC signal is Gaussian-like, while the envelope of an OFDM signal depends on the particular signal constellation used. Moreover, a block of data symbols for SC systems spreads over the whole signal bandwidth, while each data symbol for OFDM systems occupies a specific subcarrier.

\footnotetext{
${ }^{1}$ Note that other frequency domain techniques for OFDM systems using FDM pilots, such as synchronization techniques, may also be applicable for SC systems.
} 
3. The fewer blocks (in a coded frame) with FDM pilots, the lower the power backoff requirement of a HPA for SC systems.

- We should always make use of the property of low PAPR of SC signals when FDM pilots are not present in a block. For example, only the first and the last block of a frame are equipped with FDM pilots for the system considered in this thesis. The rest are just blocks with regular SC signals with low PAPR. It should be noted that high PAPR of OFDM signal always exists whether FDM pilots are used or not.

4. FDSPT pilots should be considered first for their zero pilot overhead when they gives satisfactory performance requirement.

5. It is more effective to use FDSPT pilots for coded systems than for uncoded systems.

6. The model of channel estimation errors using a Gaussian random variable can be used to evaluate the system performance with channel estimator, before actually implementing the channel estimators.

7. Using multiple frames to improve the performance of channel estimation should be considered, especially for chunk size systems.

\subsection{Future Work}

In this chapter, we provide the possible extensions of our current work on frequency domain channel estimation and equalization techniques for GMC (SC and OFDM) systems for future research, which are summarized as follows:

1. Extension to channel estimation for GMC systems with FDM pilots in timevarying frequency selective MIMO fading channels. 
- Multiple input and multiple output (MIMO) systems $[114,115]$ can be used to increase the system capacity or user data rates for future wireless systems. It is intuitive to extend this work for SISO channels to MIMO channels. The key challenges include pilot overheads, robustness to in-cell and out-of-cell interference and implementation complexity.

2. Performance improvement using more complex equalization techniques.

- Iterative joint channel equalization and decoding (turbo equalization) [16, 116] can be shown to outperform IBSDFE, with higher decoding and equalization complexity. Turbo equalization with TDM pilots has been considered in [16]. It will be interesting to evaluate and compare the performance and complexity of using turbo equalization for GMC (OFDM and SC) systems using FDM pilots (especially FDSPT pilots) in MIMO channels.

3. Study of the effects of frequency offset and phase noise on B-IFDMA systems.

4. Analysis of channel estimation errors on BER performance with iterative equalization and iterative channel estimation. 


\section{Appendix A}

\section{Details of Derivation}

\section{A.1 Derivation of (3.11)}

$$
\begin{aligned}
J_{A}= & \frac{1}{M} \sum_{\ell=0}^{M-1}\left|\frac{\tilde{H}_{\ell}^{*}}{\left|\tilde{H}_{\ell}\right|^{2}+\sigma^{2}}\left(\tilde{H}_{\ell}-\Delta H_{\ell}\right)-1\right|^{2}+\frac{\sigma^{2}}{M} \sum_{\ell=0}^{M-1}\left|\frac{\tilde{H}_{\ell}^{*}}{\left|\tilde{H}_{\ell}\right|^{2}+\sigma^{2}}\right|^{2} \\
= & \frac{1}{M} \sum_{\ell=0}^{M-1}\left|\left(\frac{\left|\tilde{H}_{\ell}\right|^{2}}{\left|\tilde{H}_{\ell}\right|^{2}+\sigma^{2}}-1\right)-\frac{\Delta H_{\ell} \tilde{H}_{\ell}^{*}}{\left|\tilde{H}_{\ell}\right|^{2}+\sigma^{2}}\right|^{2}+\frac{\sigma^{2}}{M} \sum_{\ell=0}^{M-1}\left|\frac{\tilde{H}_{\ell}^{*}}{\left|\tilde{H}_{\ell}\right|^{2}+\sigma^{2}}\right|^{2} \\
= & \frac{1}{M} \sum_{\ell=0}^{M-1}\left[\left|\frac{\left|\tilde{H}_{\ell}\right|^{2}}{\left|\tilde{H}_{\ell}\right|^{2}+\sigma^{2}}-1\right|^{2}-2 \Re\left\{\left(\frac{\left|\tilde{H}_{\ell}\right|^{2}}{\left|\tilde{H}_{\ell}\right|^{2}+\sigma^{2}}-1\right)^{*} \frac{\Delta H_{\ell} \tilde{H}_{\ell}^{*}}{\left|\tilde{H}_{\ell}\right|^{2}+\sigma^{2}}\right\}\right] \\
& +\frac{1}{M} \sum_{\ell=0}^{M-1}\left|\frac{\Delta H_{\ell} \tilde{H}_{\ell}^{*}}{\left|\tilde{H}_{\ell}\right|^{2}+\sigma^{2}}\right|^{2}+\frac{\sigma^{2}}{M} \sum_{\ell=0}^{M-1}\left|\frac{\tilde{H}_{\ell}^{*}}{\left|\tilde{H}_{\ell}\right|^{2}+\sigma^{2}}\right|^{2} \\
& \frac{\sigma^{2}}{M} \sum_{\ell=0}^{M-1} \frac{1}{\left|\tilde{H}_{\ell}\right|^{2}+\sigma^{2}} \\
& +\frac{1}{M} \sum_{\ell=0}^{M-1}\left[2 \Re\left\{\frac{\sigma^{2} \Delta H_{\ell} \tilde{H}_{\ell}^{*}}{\left(\left|\tilde{H}_{\ell}\right|^{2}+\sigma^{2}\right)^{2}}\right\}+\left|\frac{\Delta H_{\ell} \tilde{H}_{\ell}^{*}}{\left|\tilde{H}_{\ell}\right|^{2}+\sigma^{2}}\right|^{2}\right]
\end{aligned}
$$




\section{A.2 Derivation of (3.15)}

$$
\begin{aligned}
& E\left\{|\kappa|^{2}\right\}=E\left\{\left|\frac{1}{M} \sum_{\ell=0}^{M-1} \frac{\left|\tilde{H}_{\ell}\right|^{2}-\Delta H_{\ell} \tilde{H}_{\ell}^{*}}{\left|\tilde{H}_{\ell}\right|^{2}+\sigma^{2}}\right|^{2}\right\} \\
& =E\left\{\left(\frac{1}{M} \sum_{\ell=0}^{M-1} \frac{\left|\tilde{H}_{\ell}\right|^{2}-\Delta H_{\ell} \tilde{H}_{\ell}^{*}}{\left|\tilde{H}_{\ell}\right|^{2}+\sigma^{2}}\right)\left(\frac{1}{M} \sum_{k=0}^{M-1} \frac{\left|\tilde{H}_{k}\right|^{2}-\Delta H_{k} \tilde{H}_{k}^{*}}{\left|\tilde{H}_{k}\right|^{2}+\sigma^{2}}\right)^{*}\right\} \\
& =\frac{1}{M^{2}} \sum_{\ell=0}^{M-1} E\left\{\left|\frac{\left|\tilde{H}_{\ell}\right|^{2}-\Delta H_{\ell} \tilde{H}_{\ell}^{*}}{\left|\tilde{H}_{\ell}\right|^{2}+\sigma^{2}}\right|^{2}\right\} \\
& +\frac{1}{M^{2}} \sum_{\substack{\ell=0 \\
\ell \neq k}}^{M-1} \sum_{\substack{k=0 \\
k \neq \ell}}^{M-1} E\left\{\frac{\left(\left|\tilde{H}_{\ell}\right|^{2}-\Delta H_{\ell} \tilde{H}_{\ell}^{*}\right)\left(\left|\tilde{H}_{k}\right|^{2}-\Delta H_{k}^{*} \tilde{H}_{k}^{*}\right)}{\left(\left|\tilde{H}_{\ell}\right|^{2}+\sigma^{2}\right)\left(\left|\tilde{H}_{k}\right|^{2}+\sigma^{2}\right)}\right\} \\
& =\frac{1}{M^{2}} \sum_{\ell=0}^{M-1} \frac{\left|\tilde{H}_{\ell}\right|^{2}\left(\left|\tilde{H}_{\ell}\right|^{2}+\sigma_{\Delta H}^{2}\right)}{\left(\left|\tilde{H}_{\ell}\right|^{2}+\sigma^{2}\right)^{2}}-2 \Re\left\{E\left\{\frac{\left|\tilde{H}_{\ell}\right|^{2} \cdot \Delta H_{\ell}^{*} \tilde{H}_{\ell}}{\left|\tilde{H}_{\ell}\right|^{2}+\sigma^{2}}\right\}\right\} \\
& +\frac{1}{M^{2}} \sum_{\substack{\ell=0 \\
\ell \neq k}}^{M-1} \sum_{\substack{k=0 \\
k \neq \ell}}^{M-1} \frac{\left|\tilde{H}_{\ell}\right|^{2}\left|\tilde{H}_{k}\right|^{2}-\left|\tilde{H}_{\ell}\right|^{2} E\left\{\Delta H_{k}^{*} \tilde{H}_{k}\right\}-\left|\tilde{H}_{k}\right|^{2} E\left\{\Delta H_{\ell}^{*} \tilde{H}_{\ell}\right\}}{\left(\left|\tilde{H}_{\ell}\right|^{2}+\sigma^{2}\right)\left(\left|\tilde{H}_{k}\right|^{2}+\sigma^{2}\right)} \\
& +\frac{1}{M^{2}} \sum_{\substack{\ell=0 \\
\ell \neq k}}^{M-1} \sum_{\substack{k=0 \\
k \neq \ell}}^{M-1} \frac{E\left\{\Delta H_{\ell} \Delta H_{k}^{*} \tilde{H}_{\ell} \tilde{H}_{k}\right\}}{\left(\left|\tilde{H}_{\ell}\right|^{2}+\sigma^{2}\right)\left(\left|\tilde{H}_{k}\right|^{2}+\sigma^{2}\right)} \\
& \approx \frac{1}{M^{2}} \sum_{\ell=0}^{M-1} \frac{\left|H_{\ell}\right|^{2}\left(\left|H_{\ell}\right|^{2}+\sigma_{\Delta H}^{2}\right)}{\left(\left|H_{\ell}\right|^{2}+\sigma^{2}\right)^{2}} \\
& +\frac{1}{M^{2}} \sum_{\substack{\ell=0 \\
\ell \neq k}}^{M-1} \sum_{\substack{k=0 \\
k \neq \ell}}^{M-1} \frac{\left|H_{\ell}\right|^{2}\left|H_{k}\right|^{2}}{\left(\left|H_{\ell}\right|^{2}+\sigma^{2}\right)\left(\left|H_{k}\right|^{2}+\sigma^{2}\right)}
\end{aligned}
$$

where Assumption 1b, 1c, 1d, 1e, 2 and 4 are used in the derivation. 


\section{A.3 Derivation of (4.4)}

$$
\begin{aligned}
x_{S}^{\prime}(n) & =\frac{1}{L} \sum_{\ell=0}^{M-1} A_{\ell} Q_{\ell} e^{j \frac{2 \pi n \ell}{L}} \\
& =\frac{1}{L} \sum_{\ell=0}^{M-1} A_{\ell} e^{j \frac{2 \pi n \ell}{L}}-\frac{1-\alpha}{L} \sum_{\ell=0}^{M-1} A_{\ell} \sum_{i=0}^{N-1} \delta(\ell-i K) e^{j \frac{2 \pi n \ell}{L}} \\
& =a_{n}^{\prime}-\frac{1-\alpha}{L} \sum_{r=0}^{N-1} A_{r K} e^{j \frac{2 \pi n r K}{L}} \\
& =a_{n}^{\prime}-\frac{1-\alpha}{L} \sum_{r=0}^{N-1} \sum_{m=0}^{M-1} a_{m} e^{j \frac{2 \pi m r K}{L}} e^{j \frac{2 \pi n r K}{L}} \\
& =a_{n}^{\prime}-\frac{1-\alpha}{L} \sum_{m=0}^{M-1} a_{m} \sum_{r=0}^{N-1} e^{j 2 \pi\left(\frac{n}{L}-\frac{m}{M}\right) K r} \\
& =a_{n}^{\prime}-(1-\alpha) \frac{N}{L} \sum_{m=0}^{M-1} a_{m} \frac{\operatorname{sinc}\left(\frac{n}{I}-m\right)}{\operatorname{sinc}\left(K\left(\frac{n}{L}-\frac{m}{M}\right)\right)} e^{j \pi\left(\frac{n}{L}-\frac{m}{M}\right) K(N-1)}
\end{aligned}
$$

where from (A.3) to (A.4), we use the following identity, given as

$$
\frac{1}{N} \sum_{n=0}^{N-1} e^{j 2 \pi x n}=\frac{\operatorname{sinc}(N x)}{\operatorname{sinc}(x)} e^{j \pi x(N-1)}
$$

where $x \in \mathbb{R}$. 


\section{Appendix B}

\section{Performance Comparison of IFDMA and DS-CDMA Systems in the Presence of Frequency Offsets and Phase Noise}

This appendix provides the performance comparison between interleaved frequency division multiple access (IFDMA) and direct sequence code division multiple access (DS-CDMA) systems with and without carrier frequency offsets and phase noise ${ }^{1}$. Our motivation to spread the frequencies (subcarriers) over broader bandwidth is to enhance frequency diversity, while constant interleaved distance results in better PAPR. We want to investigate the suitability of IFDMA for uplink transmission. We first describe the frequency domain LMMSE equalizer for IFDMA and DS-CDMA systems, followed by the performance comparison between IFDMA and DS-CDMA systems without frequency offsets and phase noise. An analytical result on average SNR performance for single-user IFDMA systems with constant carrier frequency offset and Wiener phase noise is also included. This result can be used as a performance bound for the IFDMA systems with frequency impairments. We then compare the performance between IFDMA and DS-CDMA multi-user systems with constant carrier frequency offset and phase noise. Finally, a brief summary is included.

\footnotetext{
${ }^{1}$ Note that we include this topic in the appendix for the coherence of the thesis. The results in this appendix can serve as one of the motivations for considering IFDMA systems in Chapter 6 .
} 


\section{B.1 Frequency Domain LMMSE Receiver for DS- CDMA and IFDMA Systems}

Consider a CDMA system with spreading factor $K_{s}$ and $M$ data symbols for the $p$ th user $\left\{a_{m}^{(p)}\right\}_{m=0}^{M-1}$, which are zero mean and normalized uncorrelated random variables. The data is transmitted in blocks and preceded by a CP so that in the receiver the circular convolution can be applied. Each of the $P_{u}$ users in the system has $N_{A}$ receiving antennas. Note that $P_{u} \leq K_{s}$. We assume the receiver is sampling at chip rate, $1 / T_{c}$, such that $M K_{s}$ received samples are obtained per block.

\section{B.1.1 Frequency Domain LMMSE Receiver for DS-CDMA Systems}

After removing the CP and taking the $M K_{s}$-point FFT of the received samples and assuming perfect synchronization, the received frequency samples from the $n$th receiving antenna can be expressed as

$$
R_{n, \ell+k M}=\sum_{i=1}^{P_{u}} A_{\ell}^{(i)} H_{n, \ell+k M}^{(i)}+V_{n, \ell+k M}
$$

where $n=1,2, \ldots, N_{A}, \ell=0,1, \ldots, M-1$ and $k=0,1, \ldots, K_{s}-1$. In vector form, the received frequency samples from the $N_{A}$ antennas can be written as

$$
\mathbf{R}_{\ell}=\sum_{i=1}^{P_{u}} A_{\ell}^{(i)} \mathbf{H}_{\ell}^{(i)}+\mathbf{V}_{\ell}
$$

where $\mathbf{R}_{\ell}=\left[R_{1, \ell}, R_{1, \ell+M}, \ldots, R_{1, \ell+\left(K_{s}-1\right) M}, \ldots, R_{N, \ell}, \ldots, R_{N, \ell+\left(K_{s}-1\right) M}\right]^{T}, \mathbf{H}_{\ell}^{(i)}=\left[H_{1, \ell}^{(i)}\right.$ $\left.H_{1, \ell+M}^{(i)}, \ldots, H_{1, \ell+\left(K_{s}-1\right) M}^{(i)}, \ldots, H_{N, \ell}^{(i)}, \ldots, H_{N, \ell+\left(K_{s}-1\right) M}^{(i)}\right]^{T}$ is the channel frequency response for the $i$ th user and $\mathbf{V}_{\ell}=\left[V_{1, \ell}, V_{1, \ell+M}, \ldots, V_{1, \ell+\left(K_{s}-1\right) M}, \ldots, V_{N, \ell}, \ldots, V_{N, \ell+\left(K_{s}-1\right) M}\right]^{T}$ is the corresponding noise samples. Assuming $p$ th user is the desired user, the frequency domain LMMSE equalizer taps can be found by solving

$$
\frac{1}{K_{s}} \sum_{i=1}^{P_{u}}\left[\mathbf{H}_{\ell}^{(i)} \mathbf{H}_{\ell}^{(i) H}+\sigma^{2} \mathbf{I}\right] \mathbf{W}_{\ell}=\mathbf{H}_{\ell}^{(p)}
$$


where $\mathbf{W}_{\ell}=\left[W_{1, \ell}, W_{1, \ell+M}, \ldots, W_{1, \ell+\left(K_{s}-1\right) M}, \ldots, W_{N, \ell}, \ldots, W_{N, \ell+\left(K_{s}-1\right) M}\right]^{T}$, and the MMSE can be expressed as

$$
J_{D}^{(p)}=1-\frac{1}{M K_{s}} \sum_{\ell=0}^{M-1} \mathbf{W}_{\ell}^{H} \mathbf{H}_{\ell}^{(p)} .
$$

Solving (B.3), the optimum equalizer taps can be found as

$$
\mathbf{W}_{\ell}=\sum_{i=1}^{P_{\varkappa}} B_{\ell}^{(i)} \mathbf{H}_{\ell}^{(i)}
$$

where $\mathbf{Q}_{\ell} \mathrm{B}_{\ell}=\left[\mathbf{0}_{1 \times(p-1)}, 1, \mathbf{0}_{1 \times\left(P_{u}-p-1\right)}\right]^{T}, \mathbf{Q}_{\ell} \in \mathbb{C}^{P_{u} \times P_{u}}$ with $(i, j)$ th element being

$$
q(i, j)=\frac{1}{K_{s}} \mathbf{H}_{\ell}^{(i) H} \mathbf{H}_{\ell}^{(j)}+\sigma^{2} \mathbf{I}
$$

and $\mathbf{B}_{\ell}=\left[B^{(1)}, B^{(2)}, \ldots, B^{\left(P_{u}\right)}\right]^{T}$. Consider a special case when there is no interferer (i.e. $\left.P_{u}=1\right)$ and single receiving antenna $\left(N_{A}=1\right)$. The filter taps can be simplified as

$$
W_{1, \ell+k M}=\frac{H_{1, \ell+k M}^{(1)}}{\frac{1}{K_{s}} \sum_{k=0}^{K_{s}-1}\left|H_{1, \ell+k M}^{(1)}\right|^{2}+\sigma^{2}}
$$

and the corresponding MMSE is

$$
J_{D}=\frac{\sigma^{2}}{M} \sum_{\ell=0}^{M-1} \frac{1}{\frac{1}{K_{s}} \sum_{k=0}^{K_{s}-1}\left|H_{1, \ell+k M}^{(1)}\right|^{2}+\sigma^{2}}
$$

where ${ }_{D}$ stands for DS-CDMA.

\section{B.1.2 Frequency Domain LMMSE Receiver for IFDMA Sys- tems}

Assuming perfect synchronization for IFDMA systems, the received frequency samples for the $p$ th user can be expressed as

$$
\mathbf{R}_{f}= \begin{cases}\sqrt{K_{s}} \mathbf{H}_{f}^{(p)} A_{f}^{(p)}+\mathbf{V}_{f}, & f=p+\ell K_{s} \\ 0, & \text { otherwise }\end{cases}
$$

where $f=0,1, \ldots, M K_{s}-1, \mathbf{R}_{f}=\left[R_{f}^{(1)}, R_{f}^{(2)}, \ldots, R_{f}^{\left(N_{A}\right)}\right]^{T}, \mathbf{H}_{f}^{(p)}=\left[H_{f, 1}^{(p)}, H_{f, 2}^{(p)}, \ldots, H_{f, N_{A}}^{(p)}\right]^{T}$ and $\mathbf{V}_{f}=\left[V_{f}^{(1)}, V_{f}^{(2)}, \ldots, V_{f}^{\left(N_{A}\right)}\right]^{T}$. The optimum linear MMSE equalizer taps for the 
$p$ th user can be found as

$$
\mathbf{W}_{\ell}^{(p)}=\frac{1}{\sqrt{K_{s}}} \frac{\mathbf{H}_{p+\ell K_{s}}^{(p)}}{\mathbf{H}_{p+\ell K_{s}}^{(p) H} \mathbf{H}_{p+\ell K_{s}}^{(p)}+\sigma^{2}}
$$

where $\ell=0,1, \ldots, M-1$. The corresponding MMSE can then be calculated as

$$
J_{I}^{(p)}=\frac{\sigma^{2}}{M} \sum_{\ell=0}^{M-1} \frac{1}{\mathbf{H}_{p+\ell K_{s}}^{(p) H} \mathbf{H}_{p+\ell K_{s}}^{(p)}+\sigma^{2}} .
$$

When $N_{A}=1$ and $P_{u}=1,($ B.10) and (B.11) can be simplified as

$$
W_{\ell}^{(1)}=\frac{1}{\sqrt{K_{s}}} \frac{H_{p+\ell K_{s}}^{(1)}}{\left|H_{p+\ell K_{s}}^{(1)}\right|^{2}+\sigma^{2}}
$$

and

$$
J_{I}=\frac{\sigma^{2}}{M} \sum_{\ell=0}^{M-1} \frac{1}{\left|H_{p+\ell K_{s}}^{(1)}\right|^{2}+\sigma^{2}}
$$

where ${ }_{I}$ stands for IFDMA. Note that using (B.8) and (B.13), (4.17) can be used to find the average SNR for IFDMA and DS-CDMA systems using LMMSE equalizers.

\section{B.2 Comparison of the DS-CDMA and IFDMA Systems without Frequency Impairments}

This section provides the comparison between the IFDMA and DS-CDMA systems in terms of the number of users, signal to noise ratio, denoted by $E s / N o$, and number of antenna elements, without frequency offset and phase noise. For the DS-CDMA system, (B.4) is used to obtain the average SNR, while for for IFDMA system (B.11) is used. The system parameters used in the simulation are shown in Table B.1. The channel model used is the 3-path SUI-5 fixed wireless channel with large delay spread $(10 \mu s)$ [60]. 1000 channel realizations are used to obtain the average SNR.

\section{B.2.1 Effect of Number of Users, $P_{u}$}

Fig. B.1 shows the average SNR for the system using IFDMA and DS-CDMA in SUI5 channel. We observe that the performance of the IFDMA system is independent of 
Table B.1: Simulation Parameters for Evaluation of IFDMA and CDMA Systems

\begin{tabular}{|c|c|}
\hline Parameter & Value \\
\hline Chip Rate [Mcps] & 19.2 \\
\hline Spreading Factor, $K_{s}$ & 64 \\
\hline Code Index Selection & Randomly select from $\left\{0,1, \ldots, K_{s}-1\right\}$ \\
\hline Symbols per Block, $M$ & 16 \\
\hline Cyclic Prefix [samples] & 230 \\
\hline SNR [dB] & 20 \\
\hline Channel Type & SUI-5 channel $[60]$ \\
Delays [ $\mu$ s] /Power $[\mathrm{dB}]$ & {$[0410] /[0-5-10]$} \\
\hline Doppler Frequency $[\mathrm{Hz}]$ & 0 \\
\hline Channel Coding & No \\
\hline Modulation & BPSK \\
\hline Oversampling Factor & 1 \\
\hline Pulse Shaping Filter & Rectangular \\
\hline Number of Channel Realizations & 1000 \\
\hline
\end{tabular}

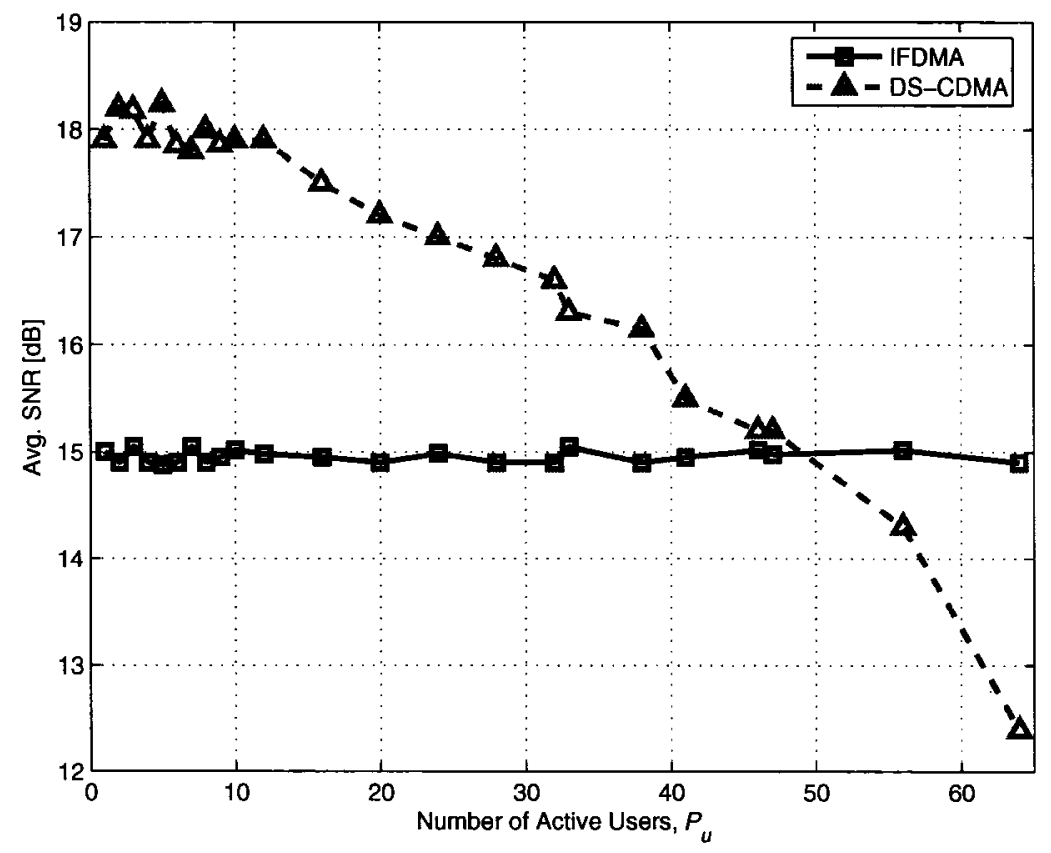

Figure B.1: Effect of the Number of Users $P_{u}$ on Avg. $S N R$ for IFDMA and DS-CDMA Systems in SUI5 Channel $\left(E s / N o=20[\mathrm{~dB}], K_{s}=64, M=16\right)$ 
the number of users in the system, $P_{u}$, which is consistent with (B.11). Moreover, for lower number of users, e.g. $P_{u} \leq 50$, the DS-CDMA system outperforms the IFDMA system. As the number of user increases, the performance of the DS-CDMA system decreases due to the interference from the other users in the system (see (B.4)). For 50 or more users, the IFDMA system outperforms the DS-CDMA system. The averaging effect (frequency diversity) of the channel frequency response accounts for the better performance of DS-CDMA system with lower number of users. This averaging effect can be seen by considering (B.8) and (B.13). From (B.8) and (B.13), if we let $K_{s}=1$ and $p=0,(\mathrm{~B} .8)$ and (B.13) are equivalent. In this case, no spreading is used and the two systems are identical. Fig. B.2 shows the effect of $K_{s}$ on the average SNR for $P_{u}=1$. As the spreading factor $K_{s}$ increases, the performance of DS-CDMA system is enhanced due to the averaging term in the denominator of (B.8). For the IFDMA system, the effect of $K_{s}$ is insignificant as there is no averaging term in the denominator of (B.13). The averaging effect becomes less significant for the DS-CDMA system when $K_{s}$ is greater than 8 for SUI-5 channel.

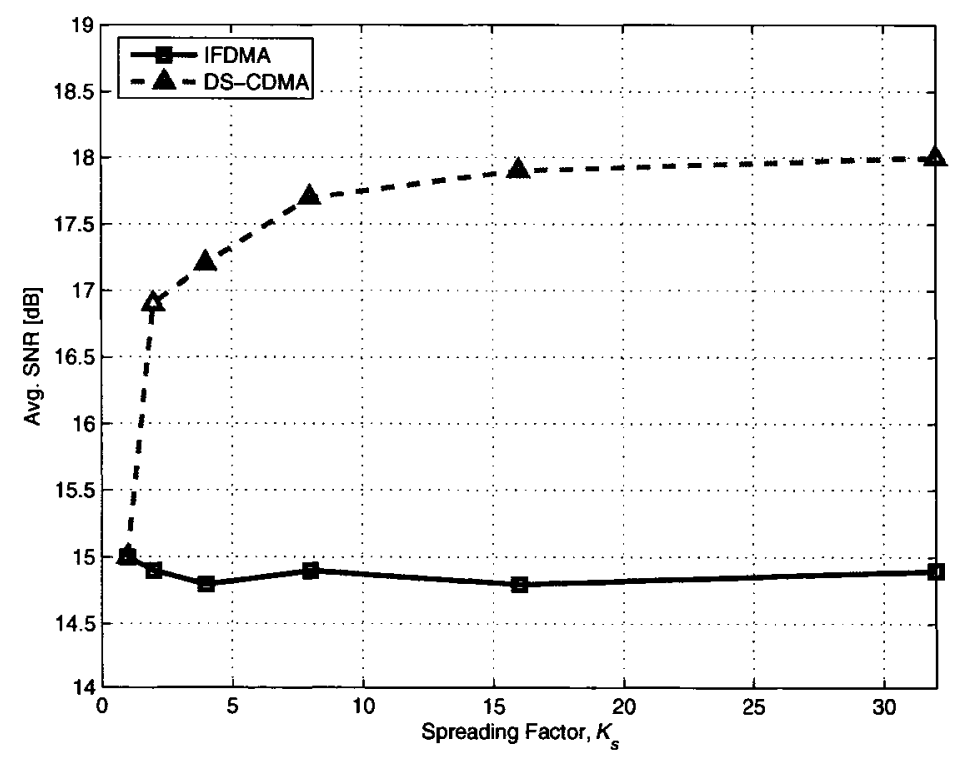

Figure B.2: Effect of $K_{s}$ on Avg. $S N R$ for IFDMA and DS-CDMA Systems in SUI5 Channel $\left(E s / N o=20[\mathrm{~dB}], P_{u}=1\right)$ 


\section{B.2.2 Effect of $E s / N o$}

Fig. B.3 depicts the average SNR for the IFDMA and DS-CDMA systems with different values of $E s / N o$ in SUI5 Channel. As $E s / N o$ increases, the absolute value of the performance difference between the IFDMA system and the DS-CDMA systems also increases for the case of single user and 64 users. For higher $E s / N o$, the difference is more obvious since at higher $E s / N o$ the averaging affect dominates that of the white Gaussian noise.

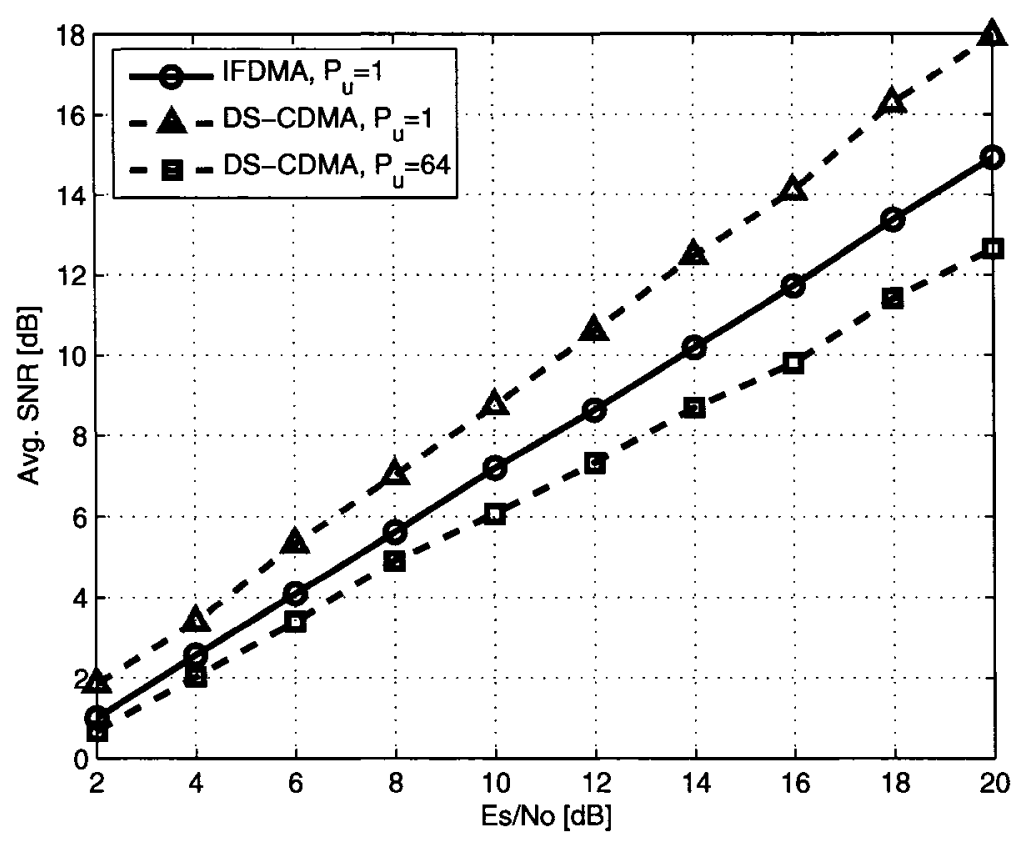

Figure B.3: Effect of $E s / N o$ on Avg. $S N R$ for IFDMA and DS-CDMA Systems in SUI5 Channel

\section{B.2.3 Effect of Number of Antenna Elements, $N_{A}$}

Fig. B.4 shows the average SNR for IFDMA and DS-CDMA systems with different number of antenna elements in SUI5 Channel, where a single user system is considered, i.e. $P_{u}=1$. For a single antenna element, the performance difference is about $3 \mathrm{~dB}$, while for two antenna elements the difference is reduced to about $1.5 \mathrm{~dB}$ and about $0.5 \mathrm{~dB}$ for four antenna elements. Thus, multiple antenna elements can be used 
to reduce the performance difference between the DS-CDMA and IFDMA systems for small number of users.

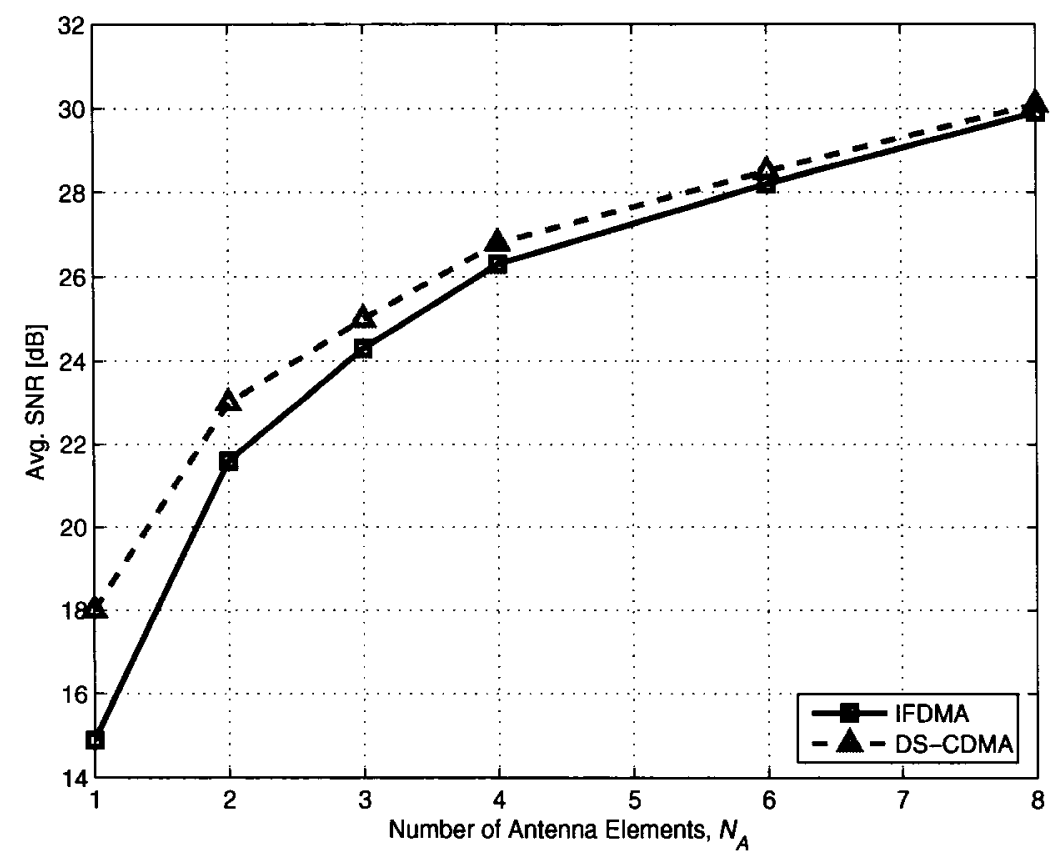

Figure B.4: Effect of $N_{A}$ on Avg. $S N R$ for IFDMA and DS-CDMA Systems in SUI5 Channel $\left(E s / N o=20[\mathrm{~dB}], P_{u}=1\right)$

\section{B.3 Analytical Results for Single-user IFDMA Sys- tems with Constant Frequency Offsets and Wiener Phase Noise}

In general, let us model the single-antenna $\left(N_{A}=1\right)$ IFDMA received signal with frequency offset or phase noise as

$$
r^{\prime}(n)=r(n) e^{j \phi(n)}, \quad n=0,1, \ldots, M K_{s}-1
$$

where $e^{j \phi(n)}$ is the time-varying frequency impairments and $r(n)$ is the $n$th received IFDMA samples without frequency impairments, given as

$$
r(n)=\sum_{p=1}^{P_{u}} \sum_{k=0}^{K_{s}-1} \frac{1}{\sqrt{K_{s}}} e^{\frac{j 2 \pi p k}{K_{s}}} \sum_{m=0}^{M-1} a_{m}^{(p)} h^{(p)}(n-m-k M)+v(n)
$$


where $h^{(p)}(n)$ is the CIR (including pulse shaping filters) of the $p$ th user and $v(n)$ is the uncorrelated noise samples. Consider the case when $P_{u}=1$ for simplicity. In the frequency domain, (B.14) can be written as

$$
\begin{aligned}
R^{\prime}(f) & =R(f) \otimes \Phi(f) \\
& =\frac{1}{M K_{s}} \sum_{\ell^{\prime}=0}^{M K_{s}-1} R\left(\ell^{\prime}\right) \Phi\left(f-\ell^{\prime}\right)
\end{aligned}
$$

where $\otimes$ represents the circular convolution operation and $\Phi(f)$ is the DFT of $e^{j \phi(n)}$, given as

$$
\Phi(f)=\sum_{n=0}^{M K_{s}-1} e^{j \phi(n)} e^{-\frac{j 2 \pi f n}{M K_{s}}} .
$$

If we sample $R^{\prime}(f)$ at $f=p+\ell K_{s}$, we have

$$
\begin{aligned}
R^{\prime}\left(p+\ell K_{s}\right) & =\frac{1}{M K_{s}} \sum_{\ell^{\prime}=0}^{M K_{s}-1}\left[S\left(\ell^{\prime}\right)+V\left(\ell^{\prime}\right)\right] \Phi\left(p+\ell K_{s}-\ell^{\prime}\right) \\
& =\frac{1}{M K_{s}} \sum_{\ell^{\prime \prime}=0}^{M-1} S\left(p+\ell^{\prime \prime} K_{s}\right) \Phi\left(\left(\ell-\ell^{\prime \prime}\right) K_{s}\right)+V^{\prime}\left(p+\ell K_{s}\right) \\
& =S\left(p+\ell K_{s}\right) \Gamma_{0}+\underbrace{\Gamma_{\ell}+V^{\prime}\left(p+\ell K_{s}\right)}_{\text {interference term }}
\end{aligned}
$$

where $\ell=0,1, \ldots, M-1, S\left(p+\ell K_{s}\right)$ is defined as,

$$
S\left(p+\ell K_{s}\right)=\sqrt{K_{s}} H\left(p+\ell K_{s}\right) A_{p+\ell K_{s}},
$$

$\Gamma_{0}$ and $\Gamma_{\ell}$ are defined as

$$
\Gamma_{0}=\Phi(0)=\frac{1}{M K_{s}} \sum_{n=0}^{M K_{s}-1} e^{j \phi(n)}
$$

and

$$
\Gamma_{\ell}=\frac{1}{M K_{s}} \sum_{\substack{\ell \prime \prime=0 \\ \ell^{\prime \prime} \neq \ell}}^{M-1} S\left(p+\ell^{\prime \prime} K_{s}\right) \Phi\left(\left(\ell-\ell^{\prime \prime}\right) K_{s}\right),
$$

respectively. From (B.18), we see that the useful data signal $S\left(p+\ell K_{s}\right)$ of the received IFDMA frequency samples with frequency impairments is first scaled by $\Gamma_{0}$ and interfered with by the other subcarriers scaled by DFT of the frequency 
impairment term $\Gamma_{\ell}$, which sometimes is called inter-carrier-interference (ICI) in the OFDM context. Note that when $\phi(n)=0$ for $n=0,1, \ldots, M K_{s}-1$, it can be shown that $\Gamma_{0}=1$ and $\Gamma_{\ell}=0$ for $\ell=1,2, \ldots, M-1$. In this case, we have $R^{\prime}\left(p+\ell K_{s}\right)=R\left(p+\ell K_{s}\right)$.

Using (B.18), we want to find the MSE of the FD-LMMSE equalizer for IFDMA systems with constant frequency impairments. Our motivation is to analytically investigate the performance degradation due to the frequency impairments if no frequency compensation is employed at the receiver. The single user case can be considered as a performance bound for $P_{u}>1$. Given the received frequency samples $R_{\ell}^{\prime}$ and the equalizer taps $W_{\ell}$, the MSE of a FD-LMMSE equalizer can be written as

$$
J_{\Delta}=\frac{1}{M^{2}} \sum_{\ell=0}^{M-1}\left\langle\left|A_{\ell}-R_{\ell}^{\prime} W_{\ell}\right|^{2}\right\rangle
$$

where $\langle\cdot\rangle$ is either time or ensemble average operator. Substituting (B.12) and (B.18) into (B.22) and assuming that the channel frequency response is deterministic, (B.22) can be expanded and simplified as

$$
\begin{array}{r}
J_{\Delta}=\frac{1}{M} \sum_{\ell=0}^{M-1}\left|H\left(p+\ell K_{s}\right)\right|^{2} B_{\ell}^{2}\left(\left|H\left(p+\ell K_{s}\right)\right|^{2}\left\langle\left|\Gamma_{0}\right|^{2}\right\rangle+\sigma^{2}+\frac{1}{M K_{s}}\left\langle\left|\Gamma_{\ell}\right|^{2}\right\rangle\right) \\
-2\left|H\left(p+\ell K_{s}\right)\right|^{2} B_{\ell} \Re\left\{\left\langle\Gamma_{0} \mid\right\rangle+1\right\}
\end{array}
$$

where $\Re\{z\}$ is the real part of $z$,

$$
\left\langle\left|\Gamma_{\ell}\right|^{2}\right\rangle=\frac{1}{M K_{s}} \sum_{\substack{\ell^{\prime \prime}=0 \\ \ell \neq \ell^{\prime \prime}}}^{M-1}\left|H\left(p+\ell K_{s}\right)\right|^{2}\left\langle\left|\Phi\left(\left(\ell-\ell^{\prime \prime}\right) K_{s}\right)\right|^{2}\right\rangle
$$

where $\Phi(\ell)$ is defined in (B.17) and

$$
B_{\ell}=\frac{1}{\left|H\left(p+\ell K_{s}\right)\right|^{2}+\sigma^{2}}
$$

Consider the simple AWGN channel. (B.23) can be simplified into

$$
\begin{aligned}
J_{\Delta}^{A W G N} & =\frac{1}{M} \sum_{\ell=0}^{M-1} \frac{1}{\left(1+\sigma^{2}\right)^{2}}\left(\left\langle\left|\Gamma_{0}\right|^{2}\right\rangle+\sigma^{2}+\frac{1}{M K_{s}}\left\langle\left|\Gamma_{\ell}^{\prime}\right|^{2}\right\rangle\right)-\frac{2 \Re\left\{\left\langle\Gamma_{0}\right\rangle\right\}}{1+\sigma^{2}}+1 \\
& =\frac{\left\langle\left|\Gamma_{0}\right|^{2}\right\rangle+\sigma^{2}}{\left(1+\sigma^{2}\right)^{2}}-\frac{2 \Re\left\{\left\langle\Gamma_{0}\right\rangle\right\}}{1+\sigma^{2}}+\frac{1}{M^{2} K_{s}\left(1+\sigma^{2}\right)^{2}} \sum_{\ell=0}^{M-1}\left\langle\left|\Gamma_{\ell}\right|^{2}\right\rangle+1
\end{aligned}
$$


where

$$
\begin{aligned}
\left\langle\left|\Gamma_{\ell}\right|^{2}\right\rangle & =\frac{1}{M K_{s}} \sum_{\substack{\ell^{\prime \prime}=0 \\
\ell \neq \ell^{\prime \prime}}}^{M-1}\left\langle\left|\Phi\left(\ell-\ell^{\prime \prime}\right)\right|^{2}\right\rangle \\
& =1+\sum_{\substack{\ell^{\prime \prime}=0 \\
\ell \neq \ell^{\prime \prime}}}^{M-1} \sum_{\substack{n=0 \\
n \neq n^{\prime}}}^{M K_{s}-1} \sum_{\substack{n^{\prime}=0 \\
n^{\prime} \neq n}}^{M K_{s}-1}\left\langle e^{j\left(\phi(n)-\phi\left(n^{\prime}\right)\right)}\right\rangle e^{\frac{-j 2 \pi\left(n-n^{\prime}\right)\left(\ell-\ell^{\prime \prime}\right)}{M}}
\end{aligned}
$$

From (B.23) and (B.26), the key to evaluate the MSE performance of the IFDMA systems with frequency impairments is to evaluate the following three ensemble averages: $\left\langle\Gamma_{0}\right\rangle,\left\langle\left|\Gamma_{0}\right|^{2}\right\rangle$ and $\left\langle\left|\Gamma_{\ell}\right|^{2}\right\rangle$. The following sub-sections calculate these assemble averages for systems with constant frequency offset and Wiener phase noise.

\section{B.3.1 Analytical Results for Constant Frequency Offset}

In the time domain, the constant carrier frequency offset can be modeled as

$$
e^{j \phi(n)}=e^{\frac{j 2 \pi \Delta f n}{M K_{s}}}, \quad n=0,1, \ldots, M K_{s}-1
$$

where $\Delta f=\Delta F_{c} \times M K_{s} T_{c}$ is the normalized constant carrier frequency offset with respect to the block duration without $\mathrm{CP}, \Delta F_{c}$ is the frequency offset in $\mathrm{Hz}$ and $T_{c}$ is the chip duration. Note that a zero phase shift at the beginning of the processing block is assumed. Using (B.29), the assemble averages for (B.26) can be calculated as the follows:

$$
\begin{gathered}
\left\langle\Gamma_{0}\right\rangle=\frac{1}{M K_{s}} \sum_{n=0}^{M K_{s}-1}\left\langle e^{\frac{j 2 \pi \Delta f n}{M K_{s}}}\right\rangle \\
=\frac{\operatorname{sinc}(\Delta f)}{\operatorname{sinc}\left(\frac{\Delta f}{M K_{s}}\right)} e^{\frac{j \pi \Delta f\left(M K_{s}-1\right)}{M K_{s}}}, \\
\left\langle\left|\Gamma_{0}\right|^{2}\right\rangle=\left\langle\left(\frac{1}{M K_{s}} \sum_{n=0}^{M K_{s}-1} e^{\frac{j 2 \pi \Delta f n}{M K_{s}}}\right)\left(\frac{1}{M K_{s}} \sum_{n=0}^{M K_{s}-1} e^{\frac{j 2 \pi \Delta f n}{M K_{s}}}\right)^{*}\right\rangle \\
=\frac{1}{M^{2} K_{s}^{2}}\left|\frac{\operatorname{sinc}(\Delta f)}{\operatorname{sinc}\left(\frac{\Delta f}{M K_{s}}\right)}\right|^{2},
\end{gathered}
$$


and

$$
\begin{aligned}
\left\langle\left|\Gamma_{\ell}\right|^{2}\right\rangle & =1+\sum_{\substack{\ell^{\prime \prime}=0 \\
\ell \neq \ell^{\prime \prime}}}^{M-1} \sum_{\substack{n=0 \\
n \neq n^{\prime}}}^{M K_{s}-1} \sum_{\substack{n^{\prime}=0 \\
n^{\prime} \neq n}}^{M K_{s}-1}\left\langle e^{j\left(\phi(n)-\phi\left(n^{\prime}\right)\right)}\right\rangle e^{\frac{-j 2 \pi\left(n-n^{\prime}\right)\left(\ell-\ell^{\prime \prime}\right)}{M}} \\
& =1+\sum_{\substack{\ell^{\prime \prime}=0 \\
\ell \neq \ell^{\prime \prime}}}^{M-1} \sum_{\substack{n=0 \\
n \neq n^{\prime}}}^{M K_{s}-1} \sum_{\substack{n^{\prime}=0 \\
n^{\prime} \neq n}}^{M K_{s}-1} e^{\frac{j 2 \pi\left(n-n^{\prime}\right)\left(\Delta f-\ell^{\prime \prime}-\ell\right)}{M}}
\end{aligned}
$$

Substituting (B.30), (B.31) and (B.32) into (B.26), we have the MSE performance for single user IFDMA system with constant carrier frequency offset in AWGN channel. Fig. B.5 shows the consistency between the theoretical results and the simulation results for a single-user IFDMA system with constant frequency offset in AWGN channel. For constant frequency offset, the effect of block size $M K_{s}$ is insignificant.

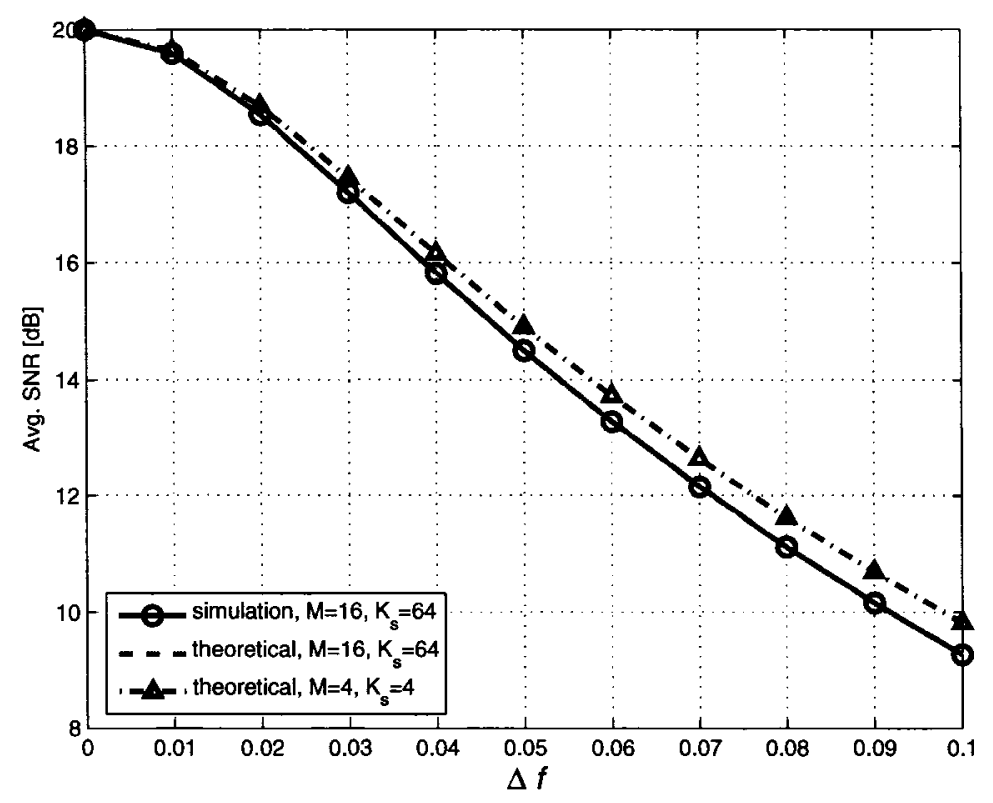

Figure B.5: Comparison of the Theoretical Results with the Simulation Results for IFDMA System with Constant Frequency Offset in AWGN Channel $\left(E s / N o=20[\mathrm{~dB}], P_{u}=1\right)$

\section{B.3.2 Analytical Results for Wiener Phase Noise}

The phase noise is the difference between the phase of the carrier and the phase of the local oscillator and can be modeled as a continuous-path Brownian motion (WienerLévy) process $[32,91,103]$ with zero mean and variance $2 \pi \beta_{L}|t|$. The corresponding 
power spectral density (PSD) is the Lorentzian PSD spectrum of the free-running carrier generator with the $3-\mathrm{dB}$ bandwidth $\beta_{L}$ (linewidth of the oscillator), as shown in Fig. B.6 [103]. The discrete-time Wiener-Lévy process can be generated as

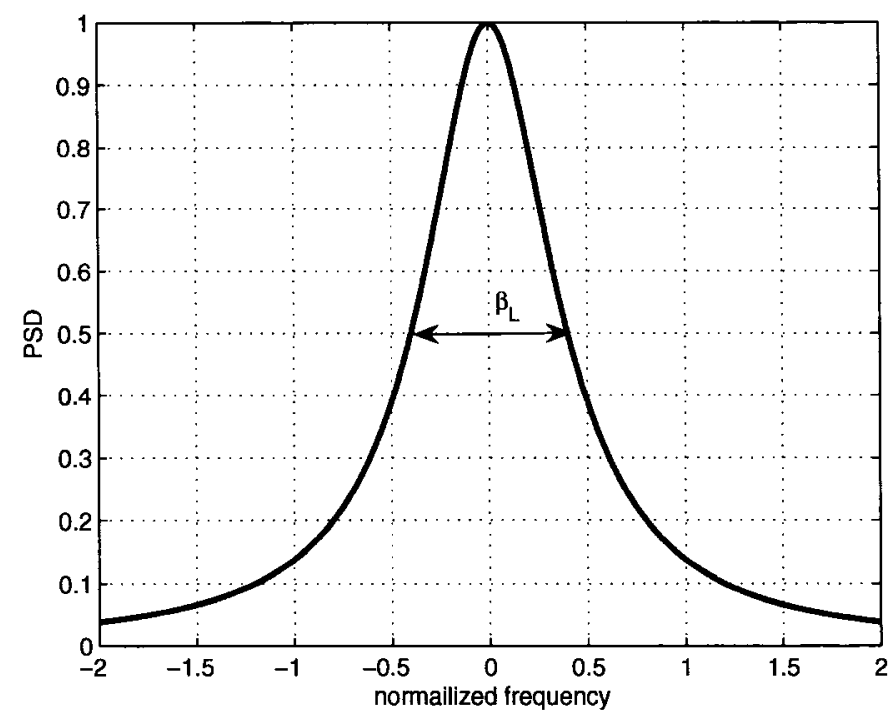

Figure B.6: Illustration of the Two-Sided 3-dB Bandwidth of the Lorentzian PSD

$$
\phi(n+1)=\phi(n)+\omega(n), \quad \phi(0)=0 \text { and } n=0,1, \ldots, M K_{s}-1
$$

where $\omega(n)$ is a Gaussian random variable with zero mean and variance $\sigma_{\omega}^{2}=2 \pi \beta_{L c}$, $\beta_{L c}=\beta_{L} T_{c}$ is the normalized 3-dB bandwidth of the PSD with respect to the chip duration $T_{c}$. From (B.33), we have the following statistics [91]:

$$
E\{\phi(n)\}=0, \quad E\left\{|\phi(n)|^{2}\right\}=2 \pi \beta_{L c}|n|
$$

and

$$
E\left\{\phi\left(n_{1}\right) \phi\left(n_{2}\right)\right\}=2 \pi \beta_{L c} \min \left\{n_{1}, n_{2}\right\} .
$$


Similar to the case of constant carrier frequency offset, using (B.33), the assemble averages for (B.26) for Wiener phase noise can be calculated as the follows:

$$
\begin{gathered}
\left\langle\Gamma_{0}\right\rangle=\frac{1}{M K_{s}} \sum_{n=0}^{M K_{s}-1}\left\langle e^{j \phi(n)}\right\rangle \\
=\frac{1}{M K_{s}} \sum_{n=0}^{M K_{s}-1} e^{-\pi \beta_{L c} n}, \\
\left\langle\left|\Gamma_{0}\right|^{2}\right\rangle=\left\langle\left(\frac{1}{M K_{s}} \sum_{n=0}^{M K_{s}-1} e^{j \phi(n)}\right)\left(\frac{1}{M K_{s}} \sum_{n=0}^{M K_{s}-1} e^{j \phi(n)}\right)^{*}\right\rangle \\
=\frac{1}{M^{2} K_{s}^{2}} \sum_{n=0}^{M K_{s}-1} \sum_{r=0}^{M K_{s}-1}\left\langle e^{j(\phi(n)-\phi(r))}\right\rangle \\
=\frac{1}{M^{2} K_{s}^{2}} \sum_{n=0}^{M K_{s}-1} \sum_{r=0}^{M K_{s}-1} e^{-\pi \beta_{L c}|n-r|}
\end{gathered}
$$

and

$$
\begin{aligned}
\left\langle\left|\Gamma_{\ell}\right|^{2}\right\rangle & =\frac{1}{M K_{s}} \sum_{\substack{\ell^{\prime \prime}=0 \\
\ell=\ell^{\prime \prime}}}^{M-1}\left\langle\left|\Phi\left(\ell-\ell^{\prime \prime}\right)\right|^{2}\right\rangle \\
& =\frac{1}{M K_{s}} \sum_{\substack{\ell^{\prime \prime}=0 \\
\ell=\ell^{\prime \prime}}}^{M-1} \sum_{n=0}^{M K_{s}-1} \sum_{r=0}^{M K_{s}-1}\left\langle e^{j(\phi(n)-\phi(r))}\right\rangle e^{-j \frac{2 \pi\left(\ell-\ell^{\prime \prime}\right)(n-r)}{M}} \\
& =\frac{1}{M K_{s}} \sum_{\substack{\ell^{\prime \prime}=0 \\
\ell \neq \ell^{\prime \prime}}}^{M-1} \sum_{n=0}^{M K_{s}-1} \sum_{r=0}^{M K_{s}-1} e^{-\pi \beta_{L c}|n-r|} e^{-j \frac{2 \pi\left(\ell-\ell^{\prime \prime}\right)(n-r)}{M}}
\end{aligned}
$$

where $\phi(n)$ is defined in (B.33). Substituting (B.36), (B.37) and (B.38) into (B.26), we have the MSE performance for single user IFDMA system with Wiener phase noise in AWGN channel. Fig. B.7 shows the performance degradation for IFDMA systems with Wiener phase noise with various block length in AWGN channel. We observe that the theoretical results are consistent with the simulation results and that the performance degradation is dependent on the block size, resulting from the increasing variance of the Wiener process as the block size increases. 


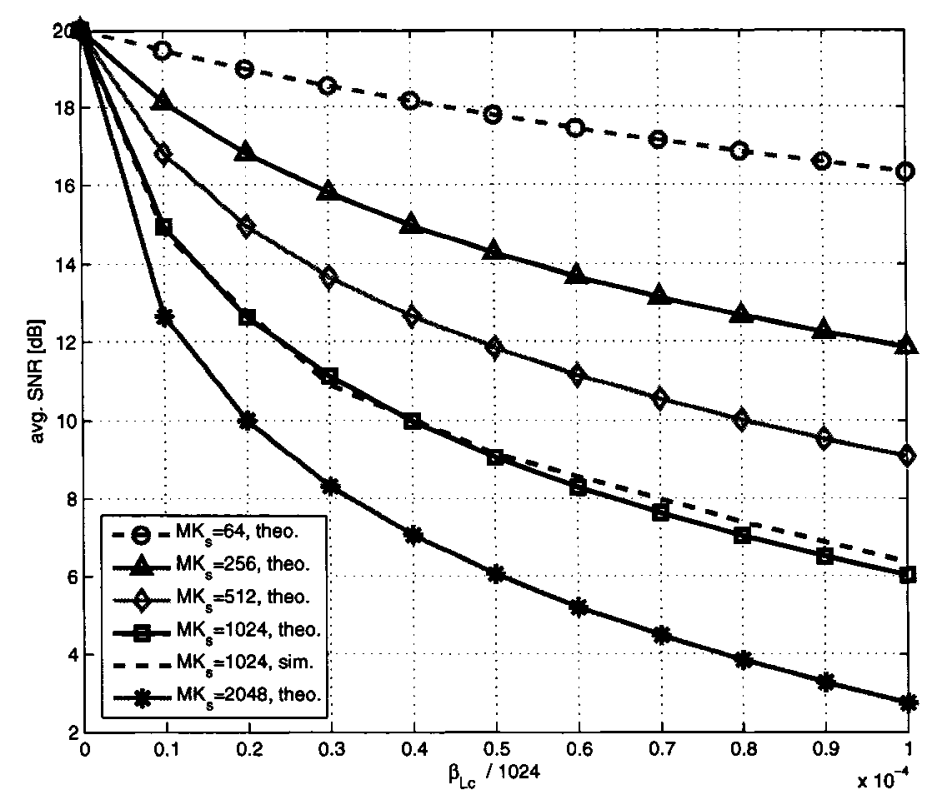

Figure B.7: Performance Degradation for IFDMA System with Wiener Phase Noise in AWGN Channel $\left(E s / N o=20[\mathrm{~dB}], P_{u}=1\right)$

\section{B.4 Comparison of IFDMA and DS-CDMA Multi- user Systems with Frequency Offset and Phase Noise}

In this section, we compare the performance degradation between the DS-CDMA and IFDMA multiuser systems with constant frequency offset and phase noise, using simulation, in AWGN channel and SUI5 channel. We assume that a FD-LMMSE equalizer is used but no adaptation of the optimum equalizer tap weights is attempted when the frequency impairments occur. In addition to the deterministic carrier frequency offset, we also model the carrier frequency offset for each user, denoted as $\Delta f_{(p)}$, as an uncorrelated Gaussian random variable with zero mean and variance $\sigma_{(p)}^{2}$. In addition to modeling the phase noise as the Wiener-Lévy process, the phase noise is also modeled as an uncorrelated Gaussian random process with zero mean and a fixed variance. In general, the received signal for the $p$ th user in multiuser systems 
with frequency impairments can be modeled as

$$
r^{\prime}(n)=\sum_{p=1}^{P_{u}} e^{j \phi_{p}(n)} \sum_{k=0}^{K_{s}-1} \frac{1}{\sqrt{K_{s}}} e^{\frac{j 2 \pi p k}{K_{s}}} \sum_{m=0}^{M-1} a_{m}^{(p)} h^{(p)}(n-m-k M)+v(n)
$$

where $e^{j \phi_{p}(n)}$ is the frequency impairments for the $p$ th user. (B.4) and (B.11) are used to obtain the average SNR.

\section{B.4.1 Comparison of Systems with Constant Frequency Off- set}

We first assume all of the users in the system have the same constant frequency offset. Fig. B.8 shows the performance for the system with constant frequency offset in AWGN channel. As the number of users increases, the performance for the IFDMA system degrades, resulting from the fact that the frequency offset destroys the orthogonality in the frequency domain for different users and more users contribute more interference to the desired reference user. For a single user system, the IFDMA

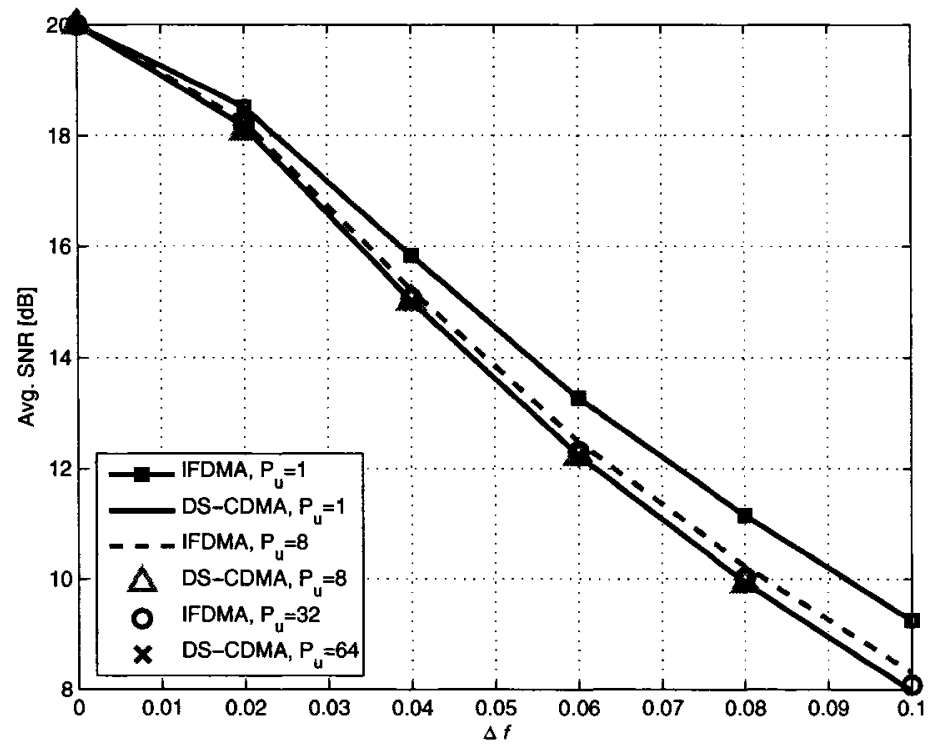

Figure B.8: Average SNR for IFDMA and DS-CDMA System with Constant Frequency Offset in AWGN Channel $\left(E s / N o=20[\mathrm{~dB}], N_{A}=1\right)$

system outperforms the DS-CDMA system by about $0.5 \mathrm{~dB}$ at $\Delta f=0.02$ and 1.2 
$\mathrm{dB}$ at $\Delta f=0.1$. Fig. B.9 depicts the performance degradation for both systems in SUI5 channel. First consider the performance of the IFDMA system. As the number

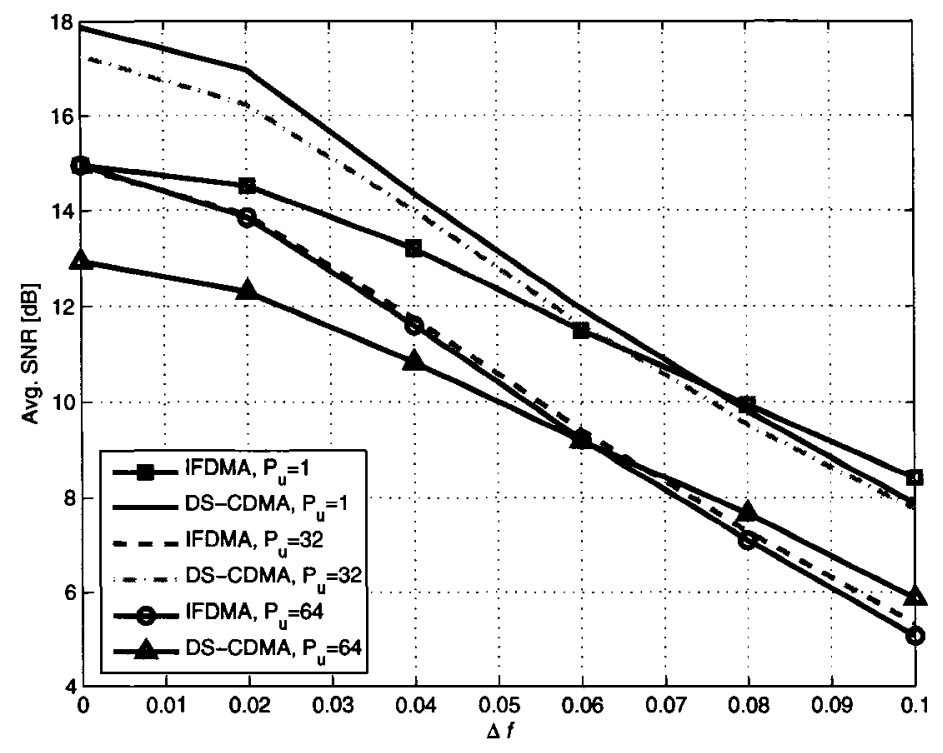

Figure B.9: Average SNR for IFDMA and DS-CDMA System with Constant Frequency Offset in SUI5 Channel $\left(E s / N o=20[\mathrm{~dB}], N_{A}=1\right)$

of users increases the performance becomes worse. Similar to the case in AWGN channel, it shows the dependency of the number of users presented in the system. For DS-CDMA system, the dependency of the number of users is not obvious.

Let us evaluate the performance degradation due to frequency offset when the offsets are modeled as uncorrelated Gaussian random variables with the same variance $\sigma_{\Delta f}^{2}$. Fig. B.10 shows such performance in AWGN channel. Similar to the previous case where all the frequency offsets for different users are the same, the IFDMA system outperforms the DS-CDMA system. Another observation for the DS-CDMA system is that the degradation is almost independent of the number of users, while for the IFDMA system the SNR performance depends on the number of users. Notice that the performance for full capacity of the IFDMA system $\left(P_{u}=K_{s}\right)$ is similar to that of the DS-CDMA system. For example, the SNR loss for both systems at the normalized standard deviation for carrier frequency offsets of $2 \%$ is about 2 dB. Fig. B.11 shows 


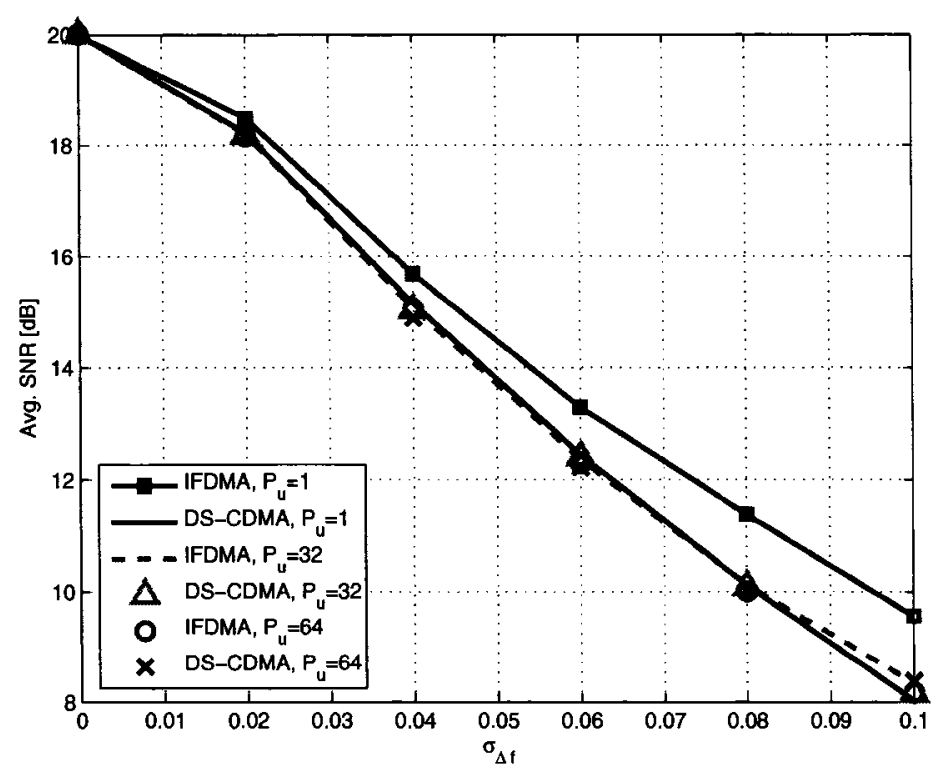

Figure B.10: Average SNR for IFDMA and DS-CDMA System with Frequency Offset Modeled as an Uncorrelated Gaussian R.V. in AWGN Channel $\left(E s / N o=20[\mathrm{~dB}], N_{A}=1\right)$

the performance for DS-CDMA and IFDMA systems with frequency offsets modeled as an uncorrelated Gaussian random variable in SUI5 channel. The performance degradation is similar to that of the systems modeled as the same constant frequency offsets (see Fig. B.9), except that the x-axis is the Gaussian standard deviation of the random variable.

\section{B.4.2 Comparison of Systems with Phase Noise}

Consider the performance of multiple user DS-CDMA and IFDMA systems with Wiener phase noise in AWGN channel, as shown in Fig. B.12. For an IFDMA system, the performance degradation due to Wiener phase noise is sensitive to the number of users, while for DS-CDMA system the performance degradation is more robust to the number of users. However, at full system capacity, both types of system have similar performance degradation due to Wiener phase noise. For the SUI-5 channel, similar conclusions can be drawn as in the case of that in AWGN channel, as shown in Fig. B.13. The DS-CDMA system with Wiener phase noise is insensitive to the number of 


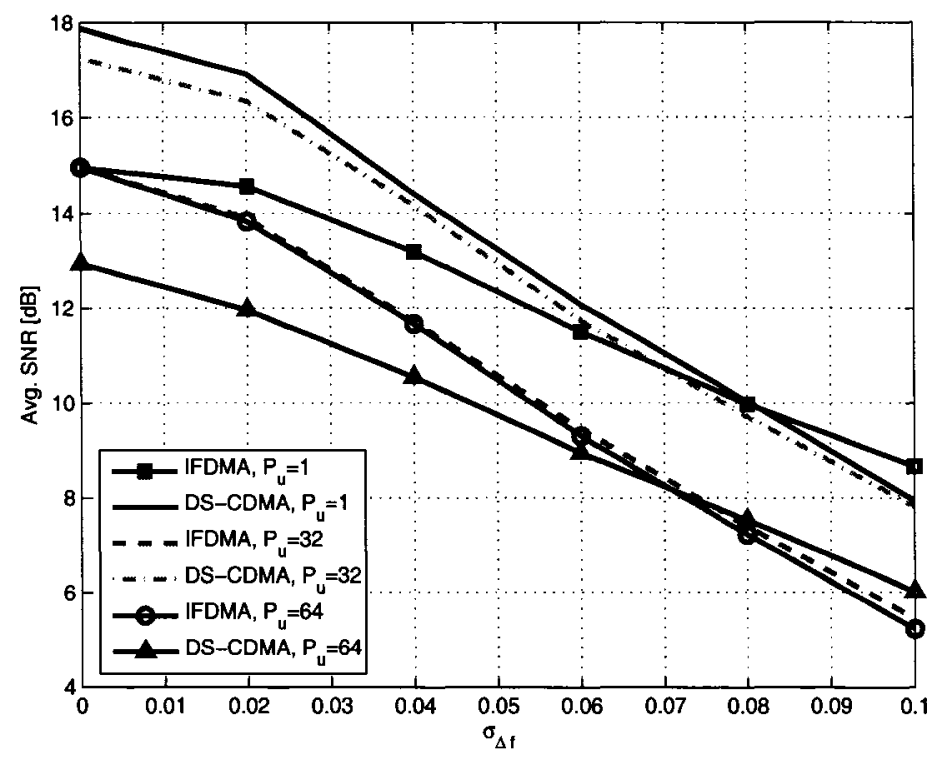

Figure B.11: Average SNR for IFDMA and DS-CDMA System with Frequency Offset Modeled as an Uncorrelated Gaussian R.V. in SUI5 Channel $\left(E s / N o=20[\mathrm{~dB}], N_{A}=1\right)$

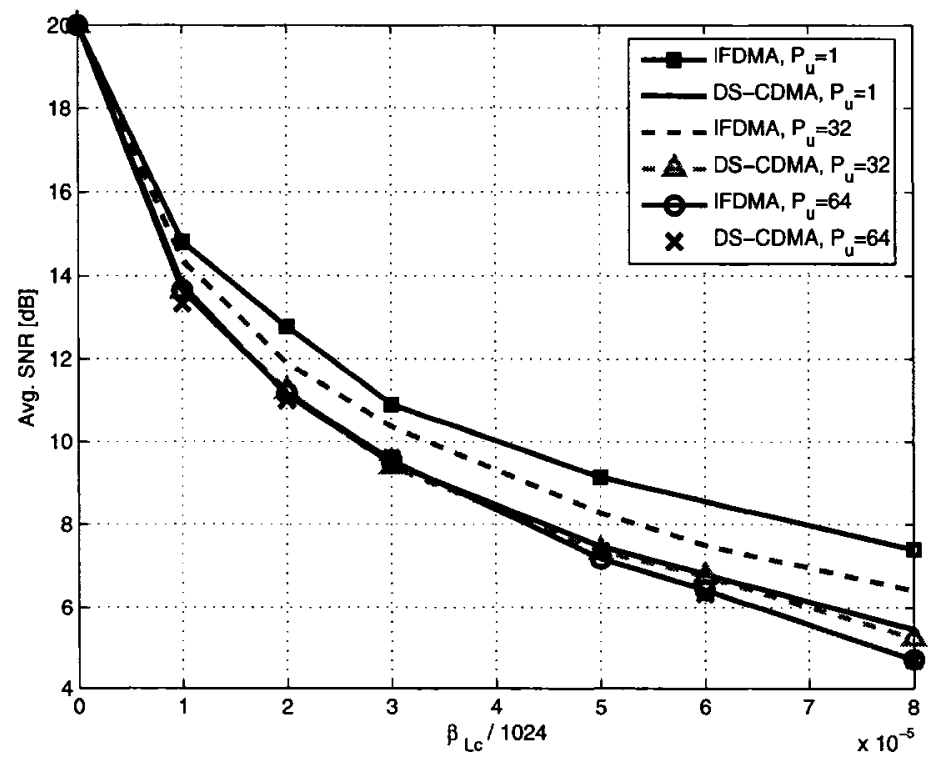

Figure B.12: Average SNR for IFDMA and DS-CDMA System with Wiener Phase Noise in AWGN Channel $\left(E s / N o=20[\mathrm{~dB}], N_{A}=1\right)$ 
active users, while the IFDMA system is more sensitive to the number of active users in the system, especially for larger values of $\beta_{L c}$. Note that there is a performance loss for DS-CDMA system when the number of users increases.

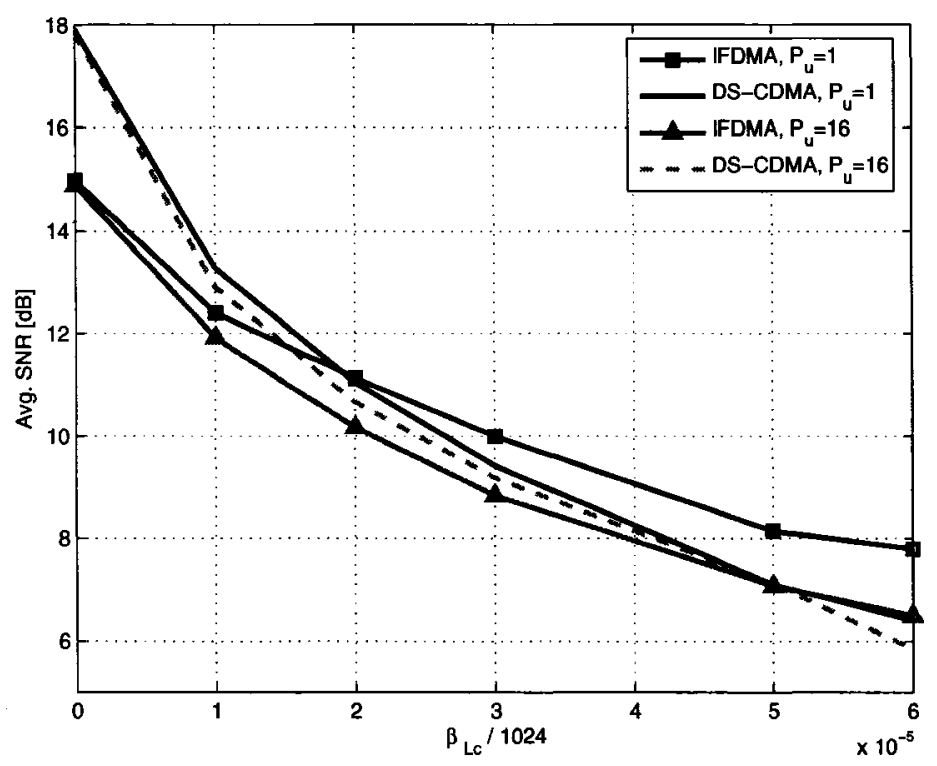

Figure B.13: Average SNR for IFDMA and DS-CDMA System with Wiener Phase Noise in SUI5 Channel $\left(E s / N o=20[\mathrm{~dB}], N_{A}=1\right)$

If the phase noise is modeled as an uncorrelated Gaussian random process with zero mean and variance $2 \pi \sigma_{P N}^{2}$ (normalized with respect to the chip duration $T_{c}$ ), the performance degradation is the same for both DS-CDMA and IFDMA systems in AWGN channel, as shown in Fig. B.14. We assume that all the users have the same value of $\sigma_{P N}^{2}$ Moreover, both systems are sensitive to the number of active users. For the SUI-5 channel, if we project the performance difference due to different schemes (IFDMA or DS-CDMA), we have similar results to that in AWGN channel, i.e. the systems have the same degradation performance of which both are sensitive to the number of active users, as shown in Fig. B.15. 


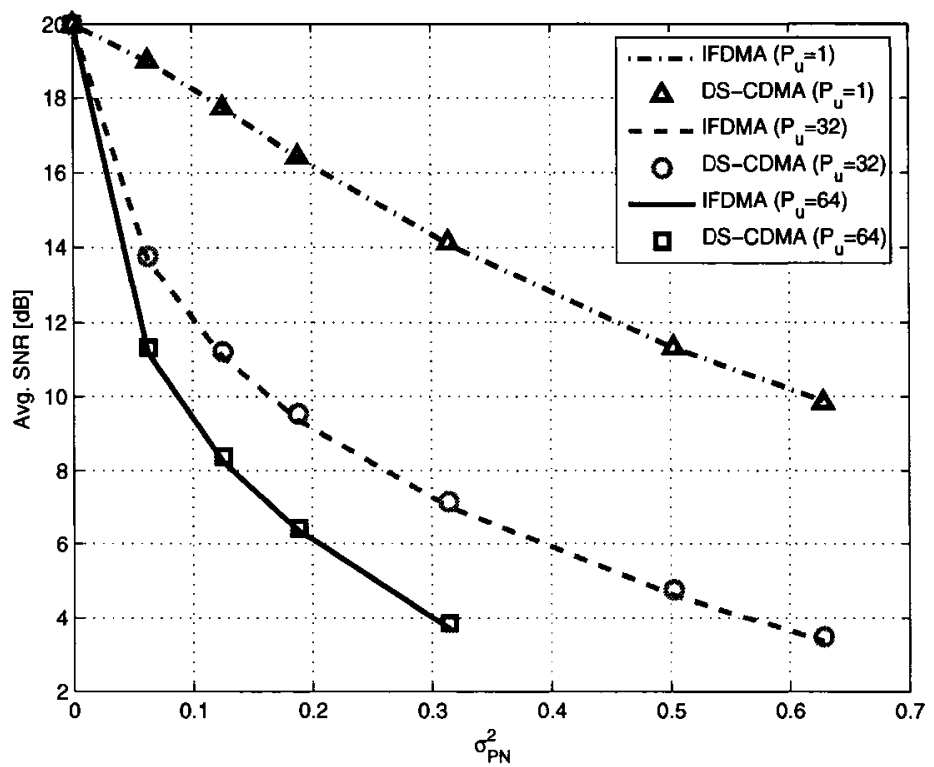

Figure B.14: Average SNR for IFDMA and DS-CDMA System with Variance of Phase Noise Modeled as an Uncorrelated Gaussian R.V. in AWGN Channel $(E s / N o=20$ [dB], $\left.N_{A}=1\right)$

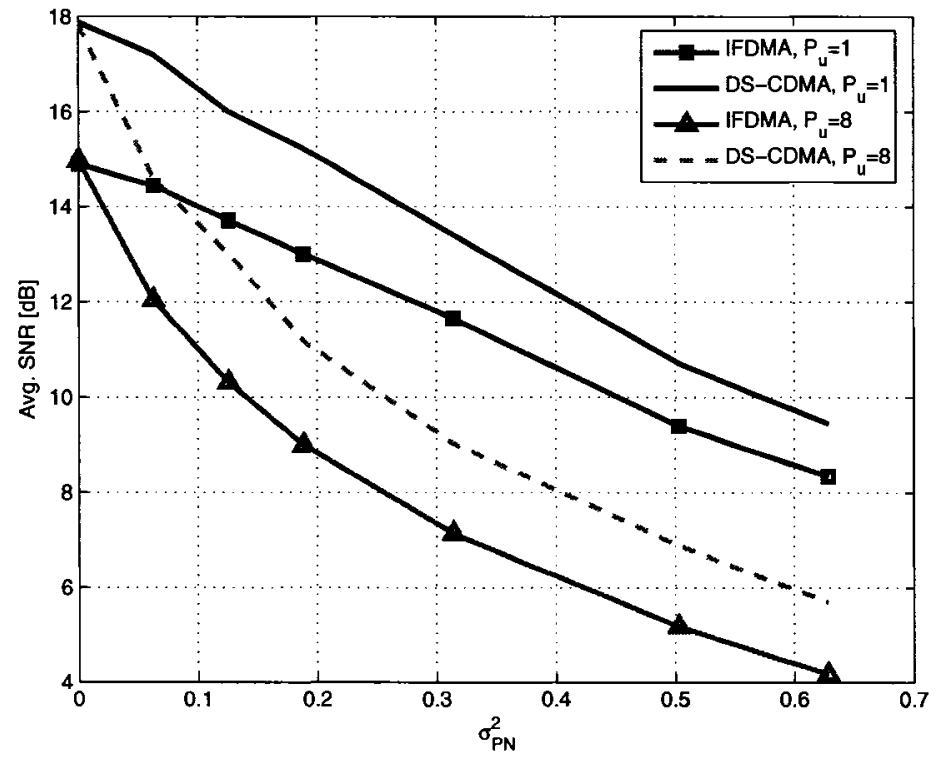

Figure B.15: Average SNR for IFDMA and DS-CDMA System with Variance of Phase Noise Modeled as an Uncorrelated Gaussian R.V. in SUI5 Channel $(E s / N o=20[\mathrm{~dB}]$, $\left.N_{A}=1\right)$ 


\section{B.5 Summary}

In this Appendix, we considered the performance comparison between IFDMA and DS-CDMA systems and channel estimation techniques for chunk size systems, where each user occupies partial system bandwidth. IFDMA signals can be considered as a special case of chunk size system with each user having $M$ smallest chunk size or with $M$ users having one smallest chunk, which is one subcarrier. Analytical results for single-user IFDMA systems with either constant frequency offset or Wiener phase noise is also presented. The results can serve as the performance bound for IFDMA systems with constant frequency offset and Wiener phase noise. For systems without frequency offset or phase noise, it was found that DS-CDMA system, in a SUI-5 channel, outperforms IFDMA system for few users in the system due to the averaging effect (frequency diversity). However, for larger number (about $3 / 4$ of the system capacity) of active users, IFDMA system outperforms the DS-CDMA system. With multiple antenna elements, the performance difference can be compensated.

For systems with constant frequency offset, in either AWGN or SUI5 channel, the IFDMA system is sensitive to the number of active users although it outperforms the DS-CDMA system when it is not at full capacity. The performance difference becomes larger when the frequency offset increases. Nevertheless, the two systems perform closely at full capacity. For systems with phase noise, depending on the phase model used, the DS-CDMA and IFDMA systems behave differently. When the phase noise is modeled as an uncorrelated Gaussian random process, the two systems have exactly the same performance, which depends on the number of active users in the system. When the phase noise is modeled as a Wiener process, the performance relations between DS-CDMA and IFDMA systems are similar to that of the systems with constant frequency offset in AWGN and SUI5 channels. 


\section{Bibliography}

[1] S. Y. Hui and K. H. Yeung, "Challenges in the migration to 4G mobile systems," IEEE Communications Magazine, vol. 41, no. 12, pp. 54-59, December 2003.

[2] E. M. Diaz, et al., "The WINNER project: research for new radio interfaces for better mobile services," in 13th IST Mobile and Wireless Communication Summit, Lyon, France, Jun. 2004.

[3] https://www.ist-winner.org/

[4] Z. Wang and G. Giannakis, "Wireless multicarrier communications where Fourier meets Shannon, IEEE Sig. Proc. Mag, vol. 17, pp. 29-48, May 2000.

[5] J. Korhonen, Introduction to $3 G$ Mobile Communications, Norwood, U.S.A., Artech House Inc., 2nd Edition, 2003.

[6] C.-T. Lam, D. Falconer, F. Danilo-Lemoine, and R. Dinis, "Channel estimation for SC-FDE systems using frequency domain multiplexed pilots," in Proc. of IEEE VTC 2006, pp. 1-5, Sep. 2006.

[7] C.-T. Lam, D. Falconer, and F. Danilo-Lemoine, "Frequency domain channel estimation for single-carrier uplink in time-varying channels," presented in $W W R F$ 16th Meeting, Apr. 2006, Shanghai, China.

[8] C.-T. Lam, D. Falconer, and F. Danilo-Lemoine, "A low complexity frequency domain iterative decision-directed channel estimation technique for single-carrier systems," in Proc. of IEEE VTC 2007, pp. 1966-1970, Apr. 2007.

[9] C.-T. Lam, G. Auer, F. Danilo-Lemoine, and D. Falconer, "Design of time and frequency domain pilots for generalized multicarrier systems," presented in IEEE ICC'07, June 24-28, 2007, Glasgow, Scotland, UK.

[10] C.-T. Lam, D. Falconer, and F. Danilo-Lemoine, "PAPR reduction using frequency domain multiplexed pilot sequences," in Proc. of IEEE WCNC 2007, pp. 1428-1432, Mar. 2007.

[11] C.-T. Lam, D. Falconer, and F. Danilo-Lemoine, "Channel estimation for subchunk-based DFT-precoded OFDM systems," presented in WWRF 18th Meeting, Jun. 2007, Helsinki, Finland.

[12] R. Dinis, C. Lam, and D. Falconer, "On the impact of phase noise and frequency offsets in block transmission CDMA schemes," in Proc. 1st International Symposium on Wireless Communication Systems, pp. 95-99, Sep. 2004. 
[13] R. Dinis, C. Lam, and D. Falconer, "A multiple access scheme for the uplink of broadband wireless systems," in Proc. IEEE GLOBECOM'04, pp. 3808-3812, Dec. 2004.

[14] R. Dinis, C. Lam, and D. Falconer, "Carrier synchronization requirements for CDMA systems with frequency-domain orthogonal signature sequence," in Proc. IEEE 8th International Symposium on Spread Spectrum Techniques and Applications, pp. 821-825, Sep. 2004.

[15] R. Dinis, C.-T. Lam, and D. Falconer, "Joint frequency-domain equalization and channel estimation using implicit pilots," submitted to Globecomm'07, 2007.

[16] B. Ng, C.-T. Lam, and D. Falconer, "Turbo frequency domain equalization for single-carrier broadband wireless systems," IEEE Trans. on Wireless Communications, vol. 6, no. 2, pp. 759-767, Feb. 2007.

[17] C.-T. Lam, D. Falconer, and F. Danilo-Lemoine, "Iterative Frequency Domain Channel Estimation for DFT-precoded OFDM Systems using In-band Pilots," submitted to IEEE Journal on Selected Areas in Commun., 2007.

[18] F. Danilo-Lemoine, D. Falconer, C.-T. Lam, M. Sabbaghian, and K. Wesolowski, "Power Backoff reduction tehcniques for generalized multicarrier waveforms," to be presented in EUSIPCO Conference, Poznan, Poland, Sept. 2007.

[19] F. Danilo-Lemoine, D. Falconer, C.-T. Lam, M. Sabbaghian, and K. Wesolowski, "Power Backoff Reduction Tehcniques for Generalized Multicarrier Waveforms", submitted to EURASIP Journal on Wireless Communications and Networking, 2007.

[20] J. Bingham, "Multicarrier modulation for data transmission: An idea whose time has come", IEEE Communications Magazine, vol. 28, pp. 5-14, May 1990.

[21] R. Tafazolli (Editor), Technologies for the Wireless Future - Wireless World Research Forum (WWRF) , West Sussex, England, John Wiley \& Sons Ltd., vol. 2, 2006.

[22] R. Prasad, OFDM for Wireless Communications Systems, Boston, U.S.A., Artech House, 2004.

[23] G. Klang, et al., "Identification of radio-link technologies," in WINNER Deliverable D2.1, Jul. 2004. Available: https://www.istwinner.org/DeliverableDocuments/D2.1.pdf

[24] H. Sari, G. Karam, and I. Jeanclaude, "Frequency-domain equalization of mobile radio and terrestrial broadcast channels", in Proc. IEEE Globecom, vol. 1, San Franciso, CA, pp. 1-5, Nov.-Dec. 1994.

[25] N. Wang and S. Blostein, "Comparison of CP-based single carrier and OFDM with power allocation," IEEE Trans. on Comm., vol. 53, no. 3, pp. 391-394, March 2005.

[26] G. Caire and G. Biglieri, "Bit-interleaved coded modulation," IEEE Trans. Inf. Theory, pp. 927-946, May 1998. 
[27] E. Zehevi, "8-PSK trellis codes for a Rayleigh channel," IEEE Trans. on Comm., vol. 40, pp. 873-884, May 1992.

[28] D. Falconer, S. Ariyavisitakul, A. Benyamin-Seeyar, and B. Eidson, "Frequency domian equalication for single-carrier broadband wireless systems," IEEE Commun. Mag., vol. 40, No. 4, pp.58-66, April, 2002.

[29] D. Falconer, S. Ariyavisitakul, A. Benyamin-Seeyar, and B. Eidson, "White paper: Frequency domain equalization for single-carrier broadband wireless systems," Tech. Rep., Feb. 2002. [Online]. Available: http://www.sce.carleton.ca/bbw/papers/whitepaper2.pdf

[30] P. Montezuma and A. Gusmão, "A pragmatic coded modulation choice for future broadband wireless communications," Proc. VTC 2001 Spring, Rhodes, Island, May, vol. 2, pp. 1324-1328, 2, 2001.

[31] J. Tubbax, B. Come, L. Van der Perre, L. Deneire, S. Donnay, and M. Engels, "OFDM versus single carrier with cyclic prefix: A system-based comparison for binary modulation," in Proc. VTC 2001 Fall, vol. 2, pp. 1115 - 1119, Oct. 2001 .

[32] T. Pollet, M. Van Bladel, and M. Moeneclaey, "BER sensitivity of OFDM systems to carrier frequency offset and Wiener phase noise," IEEE Trans. on Comm., vol. 43, pp. 191-193, Feb./Mar./Apr. 1995.

[33] J. Louveaux, L. Vandendorpe, and T. Sartenaer, "Cyclic prefixed single carrier and multicarrier transmission: Bit rate comparison," IEEE Comm. Letters, vol. 7, no. 4, pp. 180-182, April 2003.

[34] T. Rappaport, Wireless Communications: Principles and Practice, Second Edition, Prentice-Hall, 2002.

[35] J. Proakis, Digital Communication, Fourth Edition, McGraw-Hill Higher Education, 2001.

[36] M. Jeruchim, P. Balaban, and K. Shanmugan Simulation of Communication Systems: Modeling, Methodology, and Techniques, Second Edition, Prentice-Hall, 2000 .

[37] P. Bello, "Characterization of randomly time-invariant linear channels," IEEE Trans. Comm. Syst., vol. 11, pp. 360-393, Dec. 1963.

[38] W. Jakes, Ed., Microwave Mobile Communications, New York, Wiley, 1974.

[39] Y. Li, L. Jr. Cimini, and N. Sollenberger, "Robust channel estimation for OFDM systems with rapid dispersive fading channels," IEEE Trans. on Comm., vol. 46, no. 7, pp. 902-915, July 1998.

[40] L. Hanzo, M. Münster, B. J. Choi, and T. Keller, OFDM and MC-CDMA for broadband ulti-user communications, WLANs and broadcasting, West Sussex, England, John Wiley \& Sons, Ltd., 2003.

[41] H. Sari, G. Karam, and I. Jeanclaude, "Transmission techniques for digital terrestrial TV broadcasting," IEEE Comm. Mag., vol. 33, no.2, pp. 100-109, Feb. 1995. 
[42] N. Benvenuto and S. Tomasin, "Iterative design and detection of a DFE in the frequency domain," IEEE Trans. Comm., vol. 53, no. 11, pp. 1867-1875, Nov. 2005.

[43] G. Giannakis, Y. Hua, P. Stoica, and L. Tong, editors, Signal Processing Advances in Wireless and Mobile Communications, Volume 1: Trends in Channel Estimation and Equalization, Prentice-Hall, 2001.

[44] M. Tsatsanis and G. Giannakis, "Equalization of rapidly fading channels: Selfrecovering methods," IEEE Trans. Comm., vol. 44, no. 5, pp. 619-630, May 1996.

[45] X. Ma, G. Giannakis, and S. Ohno, "Optimal training for block transmissions over doubly selective wireless fading channels," IEEE Trans. on Signal Processing, vol. 51, no. 5, pp. 1351-1366, Nov. 2003.

[46] M. Tsatsanis and Z. Xu, "Pilot symbol assisted modulation in frequency selective fading wireless channels," IEEE Trans. on Signal Processing, vol. 48, no. 8, pp. 2353-2365, Aug. 2000.

[47] J. Cavers, "An analysis of pilot symbol assisted modulation for Rayleigh fading channels," IEEE Trans. Veh. Technol., vol. 40, pp. 686-693, Nov. 1991.

[48] J. Cavers, "Pilot symbol assisted modulation and differential detection in fading and delay spread," IEEE Trans. Comm., vol. 43, pp. 2206-2212, Jul. 1995.

[49] H. Meyr, M. Moeneclaey, and S. Fechtel, Digital communication receivers : synchronization, channel estimation, and signal processing, New York, Wiley, 1998

[50] J. Coon, M. Sandell, M. Beach, and J. McGeehan, "Channel and noise variance estimation and tracking alogithms for unique-word based single-carrier systems," IEEE Trans. Wireless Comm., vol. 5, no. 6, pp. 1488-1496, Jun. 2006.

[51] L. Deneire, B. Gyselinckx, and M. Engels, "Training sequences versus cyclic prefix - A new look on single carrier communication," IEEE Comm. Letters, vol. 5 no. 9, pp. 292-294, Sep. 2001.

[52] S. Kay, Fundamentals of statistical signal processing : estimation theory, Englewood Cliffs, N.J., Prentice-Hall, 1993.

[53] N. Benvenuto and S. Tomasin, "Block iterative DFE for single carrier modulation," Electron. Lett., vol. 39, no. 19, pp. 1144-1145, Sept. 2002.

[54] B. Yang, Z. Cao, and K. Letaief, "Analysis of low-complexity windowed DFTbased MMSE channel estimator for OFDM systems," vol. 49, no. 11, pp. 19771987, Nov. 2001.

[55] S. Song, A. C. Singer, and K. M. Sung, "Soft input channel estimation for turbo equalization," IEEE Trans. Signal Processing, vol. 52, no.10, pp. 2885-2894, Oct. 2004. 
[56] F. Sanzi, S. Jelting, and J. Speidel, "A comparative study of iterative channel estimators for mobile OFDM systems," IEEE Trans. Wireless Comm., vol. 2, no. 5, pp. 849-858, Sep. 2003.

[57] L. Davis, I. Collings, and P. Hoeher, "Joint MAP equalization and channel estimation for frequency-selective and frequency-flat fast-fading channels," IEEE Transactions on Communications, vol. 49, no. 12, pp. 2106-2114, December 2001.

[58] H.-J. Su, and E. Geraniotis, "Low-complexity joint channel estimation and decoding for pilot symbol-assisted modulation and multiple differential detection detection system with correlated Rayleigh fading," vol. 50, no. 2, pp. 249-261, Feb. 2002.

[59] X. Wang and H. V. Poor, "Iterative (turbo) soft interference cancellation and decoding for coded CDMA," IEEE Trans. on Commun., vol. 47, NO. 7, pp. 1046-1061, July 1999.

[60] IEEE 802.16.3c-01/29r4, July 16, 2001.

[61] E. Zimmermann, et al., "Final Report on Link Level and System Level Channel Models," in WINNER Deliverable D5.4, Nov. 2005. Available: https://www.istwinner.org/DeliverableDocuments/D5.4.pdf

[62] M.-H. Hsieh and C.-H. Wei, "Channel estimation for OFDM systems based on comb-type pilot arrangement in frequency selective fading channels," IEEE Trans. on Consumer Electronics, vol. 44, no. 1, pp. 217-225, Feb. 1998.

[63] C. Athaudage and A. Jayalath, "Low-complexity channel estimation for wireless OFDM systems," in Proc. IEEE PIMRC 2003, Beijing, China, Sep. 2003.

[64] M.-X. Chang and Y.-T. Su, "Model-based channel estimation for OFDM signals in Rayleigh fading,", IEEE Trans. Comm., vol. 50, no. 4, pp. 540-544, Apr. 2002.

[65] M. Garcìa, S. Zazo, and J. Pàez-Borrallo, "Pilot patterns for channel estimation in OFDM," IEE Electronics Letters, vol. 36, no. 12, pp. 1049-1050, Jun. 2000.

[66] R. Nilsson, O. Edfors, M. Sandell, and P. B orjesson, "An analysis of twodimensional pilot-symbol assisted modulation for OFDM," in Proc. IEEE ICPWC'97, Mumbai (Bombay), India, pp. 71-74, 1997.

[67] E. Zimmermann, et al., "Final report on identified RI key technologies, system concept, and their assessment," in WINNER Deliverable D2.10, Dec. 2005. Available: https://www.ist-winner.org/DeliverableDocuments/D2.10.pdf

[68] G. Vivier, et al., "WINNER II test scenarios and calibration cases issue 1," in WINNER Deliverable D6.13.1, Jun. 2006. Available: https://www.istwinner.org/WINNER2-Deliverables/D6.13.1.pdf

[69] D. Falconer, S. Kaiser, editors, "Broadband frequency domain-based air interfaces for future-generation wireless systems," in WWRF Working Group 4 White Paper, Version 0.16, Feb. 18, 2005. 
[70] M. Morelli and U. Mengali, "A Comparison of pilot-aided channel estimation methods for OFDM systems," IEEE Trans. Signal Processing, vol. 49, no. 12, pp. 3065-3073, Dec. 2001.

[71] S. Haykin, Adaptive Filter Theory, Third Edition, Prentice-Hall, 1996.

[72] G. Auer, "Channel estimation for OFDM systems with multiple transmit antennas by filtering in time and frequency," in Proc. of GLOBECOM'03, Dec. 2003.

[73] Y. Li, N. Seshadri, and S. Ariyavisitakul, "Channel estimation for OFDM systems with transmitter diversity in mobile wireless channels," IEEE Journal of Selected Areas on Communications, vol. 17, pp. 461-470, Mar. 1999.

[74] P. Hoeher, S. Kaiser, and P. Robertson, "Two-dimensional pilot-symbol-aided channel estimation by Wiener filtering," in Proc. 1997 IEEE Int. Conf. Acoustics, Speech, and Signal Processing, Germany, pp. 1845-1848, April 1997 .

[75] C.-M. Chang and K.-C. Chen, "Frequency-domain approach to multiuser detection in DS-CDMA communications,", IEEE Comm. Letters, vol. 4, no. 11, pp. 331-333, Nov. 2000.

[76] Y. Wang and X. Dong, "Performance of SC-FDE in UWB Communications with Channel Estimation Errors," in Proc. IEEE PACRIM'05, pp. 21-24, Aug. 2005.

[77] R. Dinis, D. Falconer, C. T. Lam, and M. Sabbaghian, "A multiple access scheme for the uplink of broadband wireless system," in Proc. IEEE GLOBECOM Conf., Dec. 2004.

[78] U. Sorger, I. De Broeck, and M. Schnel1, "Interleaved FDMA - A new spreadspectrum multiple-access scheme," in Proc. International Communications Conference within IEEE ICC'98, vol.2, pp. 1013-1017, 1998.

[79] E. Zimmermann, et al., "Assessment of radio-link technologies," in WINNER Deliverable D2.3, Feb. 2005. Available: https://www.istwinner.org/DeliverableDocuments/D2.3.pdf

[80] P. Hoeher, "TCM on frequency selective land-mobile radio channels," in Proc. 5th Tirrenia Int. Workshop on Dig. Commun., pp. 317-328, Sep. 1991.

[81] P. Hoeher, S. Kaiser, and P. Robertson, "Pilot-smbol-aided channel estimation in time and frequency," in Proc. Communication Theory Mini-Conference (CTMC) within IEEE GLOBECOM'97, pp. 90-96, 1997.

[82] S. Coleri, M. Ergen, A. Purj, and A. Bahai, "Channel estimation techniques based on pilot arrangement in OFDM systems," IEEE Trans. Broadcasting, vol.48, pp. 223-229, Sep. 2002.

[83] J. P. McGeehan and A. J. Bateman, "Phase-locked transparent tone-in-band (TTIB): A new spectrum configuration particularly suited to the ransmission of data over ssb mobile radio networks," IEEE Trans. Comm., vol.32, pp. 81-87, Jan. 1984. 
[84] F. Davarian, "Mobile digital communications via tone calibration," IEEE Trans. Vehicular Technology, vol.36, pp. 55-62, May 1987.

[85] M. Ghogho, D. McLernon, E. A.-Hernandez, and A. Swami, "Channel estimation and symbol detection for block transmission using data-dependent superimposed training," IEEE Comm. Letters, vol.12, no.3, pp. 226-229, Mar. 2005.

[86] J. K. Tugnait and S. He, "Doubly-selective channel estimation using datadependent superimposed training and exponential bases models," in Proc. 40th Annual Conf. on Information Sciences and Systems, 2006.

[87] S. Ohno and G. Giannakis, "Optimal training and redundant precoding for block transmissions with application to wireless OFDM," IEEE Trans. Comm., vol. 50, no. 12, pp. 2113-2123, Dec. 2002.

[88] J. K. Tugnait and X. Meng, "Superimposed training for channel estimation: performance analysis, training power allocation, and frame synchronization," IEEE Trans. Signal Proc., vol. 54, no. 2, pp. 752-765, Feb. 2006.

[89] F. Chin, B. Farhang-Boroujeny, and C. Ho, "Added pilot semi-blind channel estimation scheme for OFDM in fading channels," in Proc. IEEE Globecom, vol. 5, pp. 3075-3079, 2001.

[90] H. Zhu, B. Farhang-Boroujeny, and C. Schlegel, "Pilot embedding for joint channel estimation and data detection in MIMO communication systems," IEEE Comm. Letters, vol. 7, no. 1, pp. 30-32, Jan. 2003.

[91] A. Papoulis, Probability, Random Variables, and Stochastic Processes, 3rd Edition, Singapore, McGraw-Hill Inc., 1991.

[92] G. Auer, A. Dammann, and S. Sand, "Comparison of low complexity OFDM channel estimation techniques," in Proc. of Int. OFDM Workshop, Sep. 2003.

[93] L. M. Sharif, M. G.-Alkhansari, and B. H. Khalaj, "On the Peak-to-Average Power of OFDM Signals Based on Oversampling," IEEE Trans. Comm., vol.51, pp. 72-77, Jan. 2003.

[94] F. Sanzi and J. Speidel, "An adaptive two-dimensional channel estimator for wireless OFDM with application to mobile DVB-T," IEEE Trans. Broadcasting, vol.46, pp. 128-133, Jun. 2000.

[95] P. Frenger and N. Svensson, "Decision-directed coherent detechion in multicarrier systems on Rayleigh fading channels," vol. 48, no. 2, pp. 490-498, Mar. 1999.

[96] G. Vivier, et al., "The WINNER II air interface: Refined multiple access concepts," in WINNER Deliverable D4.6.1, Nov. 2006. Available: https://www.istwinner.org/WINNER2-Deliverables/D4.6.1.pdf

[97] T. Svensson, T. Frank, D. Falconer, M. Sternad, E. Costa, and A. Klein, "BIFDMA - A power effieient multiple access scheme for non-frequency-adaptive transmission," submitted to IST Summit Conf., Budapest, July 2007. 
[98] S Schifferm uller and V. Jungnickel, "Practical channel interpolation for OFDMA," presented in IEEE GlobeCom'06, San Francisco, U.S.A., 27 Nov. - 1 Dec. 2006.

[99] J. Bonnet and G. Auer, "Chunk-based channel estimation for uplink OFDM," in Proc. IEEE VTC'06 Spring, vol. 4, pp. 1555-1559, April 2006.

[100] A. Jerri, "The Shannon Sampling Theorem - Its various extensions and applications: A tutorial review," vol. 65, no. 11, pp. 1565-1596, Nov. 1977.

[101] ETSI, 3GPP TS 25.101 V5.7.0 (2003-06).

[102] M. Spiegel, Mathematical handbook, The McGraw-Hill Companies, Inc., 37th Printing, 1997.

[103] L. Tomba and W. Krzymien, "Sensitivity of the MC-DCMA access scheme to carrier phase noise and frequency offset," IEEE Trans. Vehicular Tech., vol. 48, no. 9, pp. 1657-1665, Sep. 1999.

[104] M. Fitz and J. Seymour, "On the bit error probability of QAM modulation," International Journal of Wireless Information Networks, vol. 1, no. 2, pp. 131139, 1994.

[105] J. Armstrong, "Peak-to-Average power reduction for OFDM by repeated clipping and frequency domain filtering," Elect. Lett., vol. 38, no. 8, pp. 246-47, Feb. 2002.

[106] S. Müller and J. Huber, "OFDM with reduced peak-to-average power ratio by optimum combination of partial transmit sequences," Elect. Lett., vol. 33, no. 5, pp. 368-69, Feb. 1997.

[107] R. Bäuml, R. Fisher and J. Huber, "Reducing the peak-to-average power ratio of multicarrier modulation by selected mapping," Elect. Lett., vol. 32, no. 22, pp. 2056-57, Oct. 1996.

[108] S. H. Han and J. H. Lee, "An overview of peak-to-average power ratio reduction techniques for multicarrier transmission," IEEE Wireless Comm., vol. 12, no. 2, pp. 56-65, Apr. 2005.

[109] M. García, O. Edfors and J. Paez-Borrallo, "Peak power reduction for OFDM systems with orthogonal pilot sequences," IEEE Trans. on Wireless Comm., vol. 5, no. 1, pp. 47-51, Jan. 2006.

[110] M. Sabbaghian and D. Falconer, "Reducing required power back-off of nonlinear amplifiers in serial modulation using SLM method", in Proceeding of IEEE Vehicular Technology Conference," vol. 3, pp. 1882-1886, Sep. 2005.

[111] J. Proakis and D. Manolakis, Digital Signal Processing: Principles, Algorithms and Applications, New Jersey, U. S., Prentice Hall, Inc., 1996.

[112] D. Chu, "Polyphase codes with good periodic correlation properties", IEEE Trans. Information Theory, pp. 531-532, Jul. 1972. 
[113] C. Rapp, "Effects of HPA-nonlinearity on a 4-DPSK/OFDM-signal for a digital sound broadcasting system," in Proc. the Second European Conf. Satellite Comm., pp. 179-184, Oct. 1991.

[114] L. Zheng and D. Tse, "Diversity and multiplexing: a fundamental tradeoff in multiple-antenna channels," vol. 49, no. 5, pp. 1073-1096, May 2003.

[115] A. Paulraj, D. Gore, R. Nabar and H. Bolcskei, "An overview of MIMO communications - a key to gigabit wireless," Proceedings of the IEEE, vol. 92, no. 2, pp. 198-218, Feb. 2004.

[116] M. Tuchler, R. Koetter, and A. C. Singer, "Turbo Equalization: Principles and New Results," IEEE Trans. Comm., vol.50, no.5, pp. 754-767, May 2002. 